

\section{The dynamics of Leidenfrost drops}

Michiel Antonius Jacobus van Limbeek 


\section{Samenstelling promotiecommissie:}

Prof. dr. ir. J. W. M. Hilgenkamp (voorzitter) Prof. dr. rer. nat. D. Lohse (promotor)

Prof. dr. C. Sun (co-promotor)

Prof. dr. A.-L. Biance

Prof. dr. ir. H. J. M. ter Brake

Prof. dr. ir. N. G. Deen

Prof. dr. ir. J. H. Snoeijer
Universiteit Twente

Universiteit Twente

Tsinghua University

Université Claude Bernard Lyon 1

Univeristeit Twente

Technische Universiteit Eindhoven

Univeristeit Twente

The work in this thesis was carried out at the Physics of Fluids group of the Faculty of Science and Technology of the University of Twente. The Netherlands Organization for Scientific Research (NWO) is acknowledged for financial support (MESA+ School for Nanotechnology, grant 022.003.001).

NWO $\begin{aligned} & \text { Netherlands Organisation } \\ & \text { for Scientific Research }\end{aligned}$

Nederlandse titel:

De dynamica van Leidenfrost druppels

Publisher:

Michiel Antonius Jacobus van Limbeek, Physics of Fluids, University of Twente, P.O. Box 217, 7500 AE Enschede, The Netherlands pof.tnw.utwente.nl

Cover: Artist impression of a water drop impacting a superheated glass surface as observed from below. While the drop spreads, fragments are ejected and a wetted fingering pattern emerges on the surface. This remarkable pattern is a result of the cooling inside the glass plate (chapter 5). Back side: series of images presenting an ethanol drop impact a superheated surface while in a Leidenfrost state (chapter 3).

(C) Michiel Antonius Jacobus van Limbeek, Enschede, The Netherlands - 2017

No part of this work may be reproduced by print photocopy or any other means without the permission in writing from the publisher.

Printed by Gildeprint, Enschede, The Netherlands

ISBN: 978-90-365-4289-0 


\title{
THE DYNAMICS OF LEIDENFROST DROPS
}

\author{
PROEFSCHRIFT \\ ter verkrijging van \\ de graad van doctor aan de Universiteit Twente, \\ op gezag van de rector magnificus, \\ prof. dr. T.T.M. Palstra, \\ volgens besluit van het College voor Promoties \\ in het openbaar te verdedigen \\ op vrijdag 20 januari 2017 om 16.45 uur \\ door \\ Michiel Antonius Jacobus van Limbeek \\ geboren op 3 augustus 1988 \\ te Harderwijk
}


Dit proefschrift is goedgekeurd door de promotor:

Prof. dr. rer. nat. D. Lohse

en co-promotor:

Prof. dr. C. Sun 


\section{Contents}

1 Introduction 9

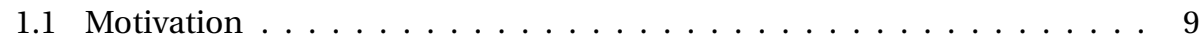

1.2 General description . . . . . . . . . . . . . . . . . 11

1.2.1 The modelling of thin vapour films with evaporation . . . . . . . . 11

1.2 .2 Pool boiling . . . . . . . . . . . . . . . . 12

1.2 .3 Static Leidenfrost drops . . . . . . . . . . . . . . . . . . 12

1.2 .4 Drop impact dynamics . . . . . . . . . . . . . . . . . . . . . . . . . . . . 13

1.2 .5 Break down of framework . . . . . . . . . . . . . . . . 14

1.3 Guide to the thesis . . . . . . . . . . . . . . . . . 14

2 Measuring thin films using Quantitative Frustrated Total Internal Reflection 17

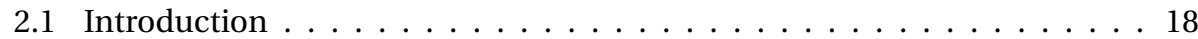

2.2 Experimental setup and image analysis . . . . . . . . . . . 18

2.2 .1 Setup ........................ 18

2.2 .2 Image calibration and analysis $\ldots \ldots \ldots \ldots$

2.2 .3 Operating conditions . . . . . . . . . . . . . . 21

2.3 Principle of quantitative height measurement

with FTIR . . . . . . . . . . . . . . . . . . . . . 21

2.3.1 When the angle of incidence is smaller than the critical angle . . . . 21

2.3.2 When the angle of incidence is larger than the critical angle . . . . . 24

2.4 Results and discussion . . . . . . . . . . . . . . . . . . . . 24

2.4.1 Reflectance by FTIR . . . . . . . . . . . . . . . . . 24

2.4.2 Validation and uncertainty analysis with a lens profile . . . . . . . . 24

2.4.3 Validation with the elastic deformation of a lens by load . . . . . . 25

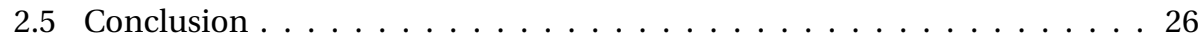

2.A Procedure guide to the analysis . . . . . . . . . . . . 29

3 Dynamic Leidenfrost effect: relevant time and length scales 33

3.1 Introduction . . . . . . . . . . . . . . . . . . . 34

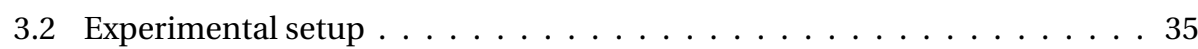

3.3 Results and Discussion . . . . . . . . . . . . . . . . . 37

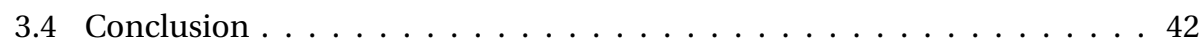


4 Boiling regimes of impacting drops on heated substrate at reduced pressure 43

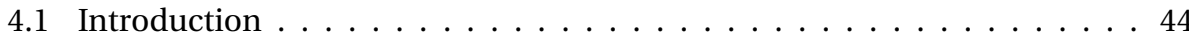

4.2 Experimental methods . . . . . . . . . . . . . . . . . . . . . . . . . . . . . . 44

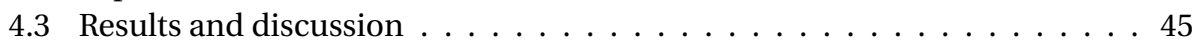

4.3 .1 Phase diagram . . . . . . . . . . . . . . . . . 45

4.3.2 Boundary between the contact and transition boiling regime . . . . 46

4.3.3 Pressure effect on the dynamic Leidenfrost temperature . . . . . . . 49

4.4 Conclusion and Outlook . . . . . . . . . . . . . . . . . . . 49

5 Cooling effects in hot substrates during drop impact 51

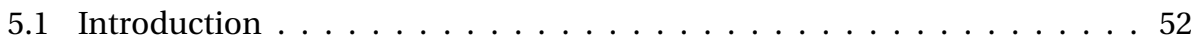

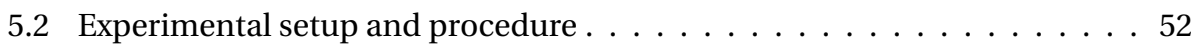

5.3 Results and discussion . . . . . . . . . . . . . . . . . . 54

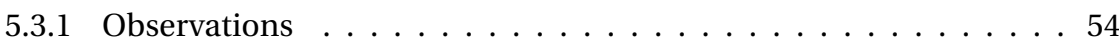

5.3 .2 Subcooling effect causes touch-down . . . . . . . . . . . . . . 59

5.3.3 Micro droplets show isothermal behaviour . . . . . . . . . . . . . . . 64

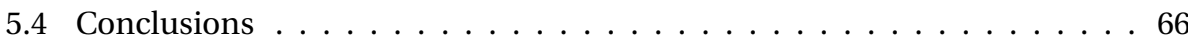

5. A Image Processing . . . . . . . . . . . . . . . . 67

5.B Error estimate of the measured height from TIR measurement . . . . . . 67

6 Spray formation 71

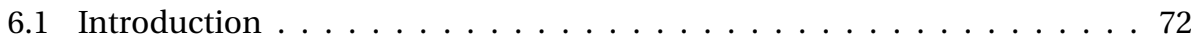

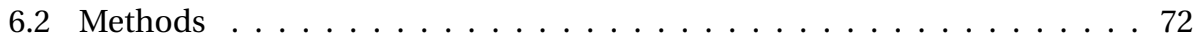

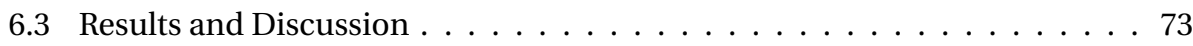

6.3 .1 Phase Diagram . . . . . . . . . . . . . . . . . 74

6.3 .2 Cooling effect . . . . . . . . . . . . . . . 77

6.3 .3 Thermal timescale . . . . . . . . . . . . . . . . 79

6.3.4 Reflection on existing literature . . . . . . . . . . . . . . . 82

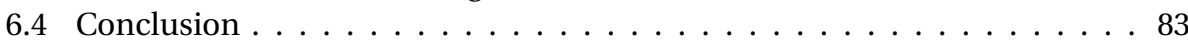

7 Surface cooling by static Leidenfrost drops $\quad 85$

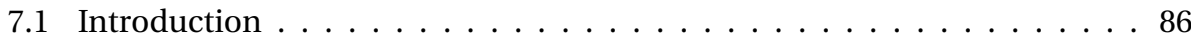

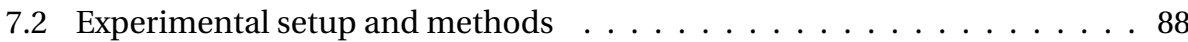

7.2 .1 Experimental setup . . . . . . . . . . . . . . . . . . . . . . .

7.2 .2 Data processing . . . . . . . . . . . . . . 88

7.3 Formulation of the theoretical model . . . . . . . . . . . . . . . . . . . . . .

7.4 Results and Discussion . . . . . . . . . . . . . . . . . . 93

7.4.1 Experimental observations and numerical validation . . . . . . . . 93

7.4 .2 Cooling strength criterion . . . . . . . . . . . . 96

7.4.3 Exploring the influence of the substrate thermal conductivity . . . . 99

7.4 .4 Regression to the isothermal model . . . . . . . . . . . . . . . 99

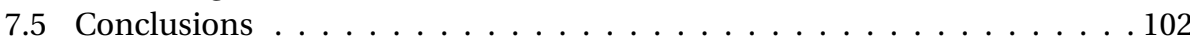

7.A Phase extraction . . . . . . . . . . . . . . . . . 104

7.B Calibration of $\mathrm{d} n / \mathrm{d} T$ and refraction correction $\ldots \ldots \ldots$. . . . . . 105

7.C Abel inversion . . . . . . . . . . . . . . . . . . . . . 107

7.D Reynolds and Péclet number estimation in the vapour film . . . . . . . . . 109

7.E Influence of the needle . . . . . . . . . . . . . . . 110 
8 Theory for Leidenfrost drops on a liquid pool 111

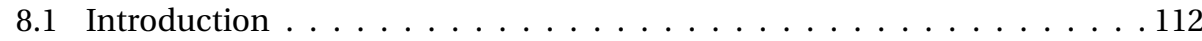

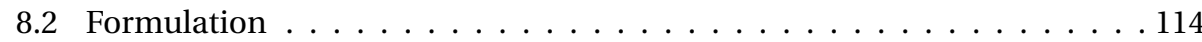

8.2 .1 Model . . . . . . . . . . . . . . . . . . . 114

8.2 .2 Structure of asymptotic analysis . . . . . . . . . . . 117

8.2 .3 Numerical solution . . . . . . . . . . . . . . . . . . . . . 117

8.3 A large drop on a pool of the same liquid . . . . . . . . . . . . . . . 118

8.3.1 Outer region 1: below the drop . . . . . . . . . . . . . . . . . . . . . . . 118

8.3.2 Outer region 2: puddle solutions . . . . . . . . . . . . . . . 120

8.3.3 Inner region: the neck profile . . . . . . . . . . . . . . . 121

8.3 .4 Summary . . . . . . . . . . . . . . . . . . . 125

8.4 Finite drop sizes and differing liquids . . . . . . . . . . . . . . . 125

8.4 .1 Finite drop size . . . . . . . . . . . . . . . . . . . . . . . . . . . . . . . . . . . . . . . . . . . .

8.4 .2 Differing liquids . . . . . . . . . . . . . . . . . . . . . . . . . . . . . . . . . . . . . . . . . . . . . .

8.5 Conclusion . . . . . . . . . . . . . . . . . . . . . . 129

$\begin{array}{ll}\text { Conclusion and Outlook } & 130\end{array}$

$\begin{array}{ll}\text { Summary } & 135\end{array}$

$\begin{array}{ll}\text { Samenvatting } & 137\end{array}$

$\begin{array}{lr}\text { Acknowledgements } & 140\end{array}$

$\begin{array}{ll}\text { About the author } & 143\end{array}$ 
CONTENTS 


\section{Chapter 1}

\section{Introduction}

\subsection{Motivation}

Temperature control is omnipresent in today's life: from keeping your fridge cold, maintaining a room at a pleasant temperature or preventing your computer from overheating. Since our body works best at $37^{\circ} \mathrm{C}$, it takes strong measures to maintain this temperature during exercising: When you sweat, small drops appear on your skin with the sole purpose to remove heat from by evaporating. The large amount of energy per liquid volume makes it a very efficient cooling method. It is therefore not surprising that the mechanism of evaporative cooling has found its way to many applications, for example in industrial processes and fire fighting. Depending on the size of the liquid body, two implementations can be distinguished: The first type is called pool boiling. Here, a hot object is submerged in a large body of liquid, for example in heat exchangers. The second type, spray cooling, cools a surface by a spray of drops.

For both types of cooling, it is important to understand the cooling efficiency for various temperatures of the solid. For pool boiling, heat is transported solely by natural convection for low plate temperatures. When the plate temperature exceeds the saturation temperature, vapour bubbles begin to nucleate on the surface, grow and detach from the plate, releasing the heat by recondensing in the bulk. The rising bubbles also induce a flow in the liquid pool and this mixing enhances the heat transfer further. At a certain temperature, the heat flux $q$ reaches a local maximum as a function of the temperature, the critical heat flux temperature (CHF), after which $q$ drops rapidly more than one order of magnitude [1], until the minimum heat flux temperature (MHF) is reached. Here, a stable vapour film has formed, separating the liquid from the solid and therefore insulating the two. This so-called dry-out phenomena is very undesirable as it limits the heat flux and thus potentially damages the heat exchangers. A typical result for the heat transfer rate is sketched in Fig. 1.1 where the heat transfer rate $q$ is shown in black as a function of the plate temperature.

A similar curve is sketch in blue for a single drop on a heated plate [2-4], as in spray cooling. The life time of the drop first decreases with increasing temperature, up to a minimum, occurring at the Nukiyama temperature. Increasing the temperature further, a sudden increase in life time is observed, first mentioned by Boerhaave [5]. Similar to 


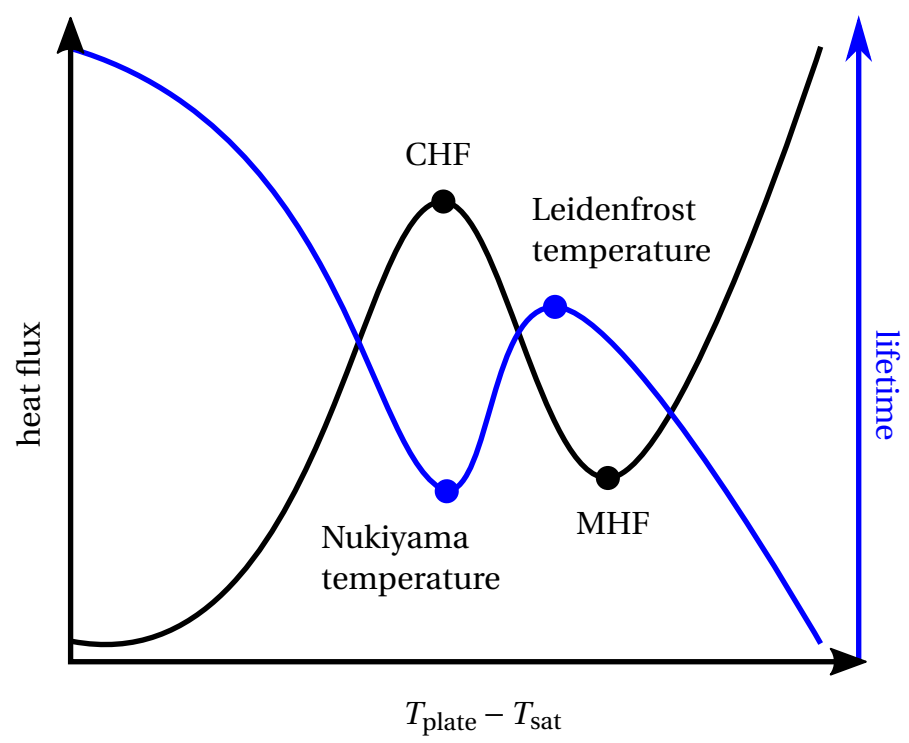

Figure 1.1: Typical curves for heat flux in pool boiling (black) and life time of a drop for spray cooling (blue) as a function of plate temperature. With increasing temperature, first the evaporation rate increases, until a maximum is reached. This is called the critical heat flux (CHF) temperature in the case of pool boiling boiling or Nukiyama temperature for drops. Increasing the temperature further leads to significant vapour film formation, isolating the drop and reducing the heat transfer rate until the minimum heat flux (MHF) temperature resp. Leidenfrost temperature is reached. After this local minimum, the heat transfer rate slowly increases, with a decrease in drop life time as a result.

the cause of the MHF-phenomenon, a vapour layer is formed, separating the drop from the plate [6]. This specific temperature is referred to as the Leidenfrost temperature $T_{\mathrm{L}}$, after the author of the first detailed study [7]. It is clear that the dry-out phenomenon and the Leidenfrost effect share similarities. The existence of the sudden decrease in cooling efficiency has large implications for a reliable temperature control in industrial processes and urges one to investigate what the dominant physical mechanisms are. It is still impossible to accurately predict the Leidenfrost temperature and how it depends on the thermal properties of both the liquid and solid, the solid roughness and ambient gas pressure. In the case of spray cooling the impact velocity of the drop plays an role as the downward momentum forces the drop into contact with the plate at a temperature higher than the Leidenfrost temperature. The lowest temperature whichs keeps the drop separated from the plate is referred to as the dynamic Leidenfrost temperature [8].

This thesis aims to investigate how the Leidenfrost temperature depends on the aforementioned parameters and study the physical mechanisms involved. The following sections will provide a general description of both pool boiling and spray cooling to enhance the readability of the thesis and demonstrate the coherence between chapters. 


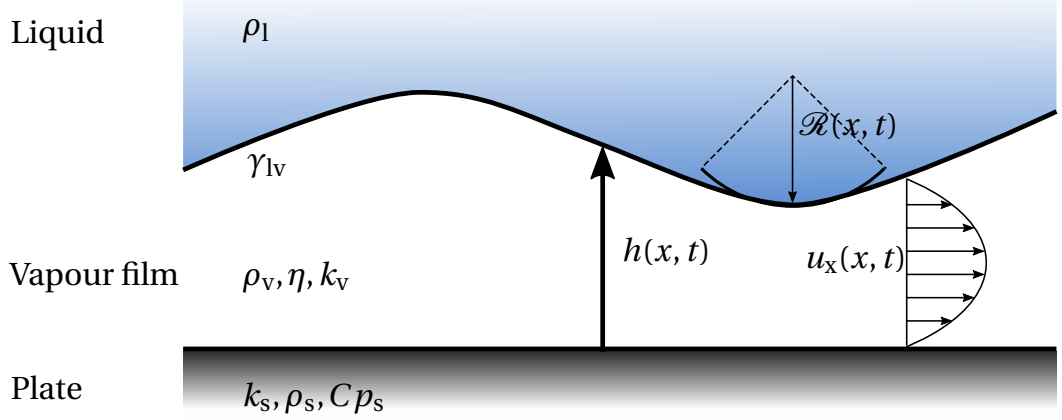

Figure 1.2: Two dimensional representation of a solid and a liquid body, separated by a vapour film of height $h$ and curvature $\kappa=1 / \mathscr{R}$. The Poiseuille flow is driven by the pressure field and replenished by the evaporation from the liquid-vapour interface.

\subsection{General description}

\subsubsection{The modelling of thin vapour films with evaporation}

The characteristic origin of the boiling crisis is the emerging vapour film, separating the liquid from the wall. Whereas the heat transfer from the wall to the liquid is characterized at small scales by a continuous temperature and heat flux across the boundary, here the vapour insulates the two bodies. The implication is twofold: first the liquid-vapour interface is now at saturation temperature and no superheating can take place. Secondly, the vapour layer has a typical thermal conductivity of $0.01 \mathrm{~W} /(\mathrm{mK})$, two orders of magnitude lower than in the case of direct liquid solid contact.

Typically, the hydrodynamic modelling of these vapour layers is based on Stokes flow as the Reynolds number is low. Since the vapour layer is thin compared to the lateral length scale, the flow normal to the wall is assumed to be negligible, justifying the use of the lubrication approximation of the Navier- Stokes equation [9]. The rigidity of the wall and the large difference in viscosity between vapour and liquids justifies the use of no-slip boundary conditions, resulting in a Poiseuille flow in the vapour layer. The problem is then closed using the continuity equation, now including a source term, $j$ describing the vapour generation at the liquid-vapour interface:

$$
\partial_{t} h+\partial_{i}\left(\frac{1}{12 \eta} h^{3} \partial_{i} P\right)=j
$$

where $h$ is the film thickness, $\partial_{t}$ and $\partial_{i}$ are the time and spatial derivatives respectively, $P$ the pressure field and $\frac{1}{12 \eta}$ the viscosity of the vapour with the mobility factor $\frac{1}{12}$ as a result of the Poiseuille flow in the gap. A sketch of a two dimensional film is presented in Fig. 1.2

The vapour generation can be described by Fourier's law:

$$
j=\frac{k_{\mathrm{v}} \Delta T}{L \rho_{\mathrm{v}}} \frac{1}{h},
$$


where $k_{\mathrm{V}}, L$ and $\rho_{\mathrm{v}}$ are the vapour thermal conductivity, enthalpy of evaporation and density respectively and $\Delta T$ the temperature difference between the wall and the saturation temperature of the liquid. In some cases, the wall temperature can locally vary significantly $[10,11]$.

The only unknown left in Equation 1.1 is the pressure field, through which the dynamics of the film interface is coupled with the dynamics inside the liquid phase. The contributions to the pressure in the film can be separated into hydrodynamic and hydrostatic ones. Where hydrodynamic ones are a result of the flow in the liquid phase, static contributions are purely described by the geometry of the film: The surface tension $\gamma$ of the film gives rise to a pressure jump across the interface proportional to its curvature $\kappa_{\mathrm{h}}: \Delta P=\gamma \kappa_{\mathrm{h}}$, while height differences in the film result in a gravitational contribution $-\left(\rho_{\mathrm{l}}-\rho_{\mathrm{v}}\right) g h$, with $\rho_{\mathrm{l}}$ being the liquid density. Influences by hydrodynamic effects are strong, decelerating or localized flows. Let us first focus on the description of the film in the case of pool boiling and drops in the absence of such hydrodynamic effects.

\subsubsection{Pool boiling}

Typically in pool boiling configurations the liquid phase is much larger than the capillary length. Therefore, the shape of the films is determined by the geometry of the solid object. In industrial applications, those solids are typically much larger than the capillary length, therefore the films are Rayleigh-Taylor unstable and bubbles are ejected at a frequency related to the average film thickness [12]. The film however is rapidly replenished at a rate that the film itself does not break up.

The onset of the formation of such a stable film is yet unclear, but recent studies gained insight that the contact-line dynamics are of great importance [13]. Furthermore, the relative long time scales involved in pool boiling allow for the development of thermal gradients inside the solid [11], resulting in hot patches where vapour pockets are present. It is suggested that eventually, these patches slowly grow over time and merge into a stable film, provided that the volume of ejected bubbles is replenished fast enough.

\subsubsection{Static Leidenfrost drops}

In the case of a single drop, the configuration studied in this thesis, the film is at most a few capillary lengths i.e. a few millimeters [4, 14-16], see Fig. 1.3.

Initially, drops can have a finite downward momentum, originating from the deposition process. This momentum however is eventually dissipated and a quasi-static configuration is reached. Quasi, meaning that the shape of the drop changes slowly in comparison with the velocity scale of the escaping vapour from underneath the drop. A pure static case is achieved if the drop is being fed from a needle, maintaining a constant drop size. Otherwise, the drop slowly boils away in a few (tens of) seconds, depending mainly on the initial drop size and the temperature of the plate $[4,15,17,18]$.

Research on the static Leidenfrost effect can roughly be divided into two categories. The first category approaches the problem from an application point of view, as it is of great importance to study the actual temperature when the phenomenon occurs, depending on the plate material, roughness and the thermal properties of the liquid. Of 


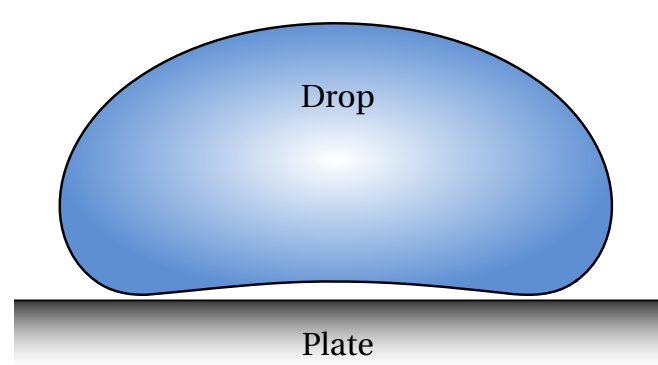

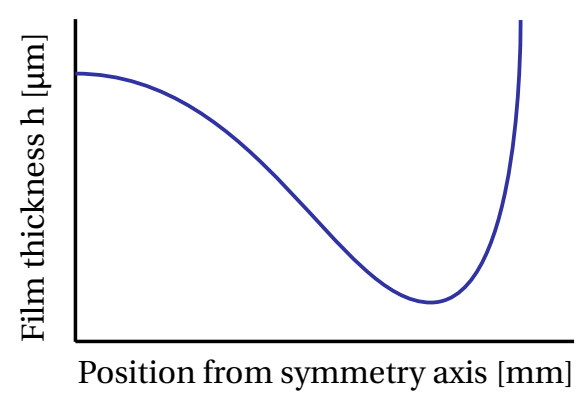

Figure 1.3: Global shape of a Leidenfrost drop (left) and shape of the vapour film (right)

great interest is the prevention of the phenomenon by texturing the plate material by micro-pillar arrays [19].

The second category focusses on addressing questions related to the shape of static Leidenfrost drops, their lifetime $[4,20,21]$ and the description of the vapour film. Drops are typically axisymmetric $[16,17]$, but in some conditions, drops can exhibit shape oscillations [22-28]. The vertical force balancing the weight of the drop is a result of the viscous shear from the escaping vapour, keeping the drop levitated. Combining Equation 1.1 and 1.2, we obtain:

$$
\partial_{t} h+\frac{1}{r} \partial_{r}\left(\frac{1}{12 \eta} r h^{3} \partial_{r}\left(k_{0}-\rho_{1} g h+\gamma \kappa_{\mathrm{h}}\right)\right)=j,
$$

where the pressure field is given by the Young-Laplace equation for the film: $\rho_{1} g h$ being the hydrostatic contribution (neglecting $\rho_{\mathrm{V}}$ ) and $\gamma \kappa_{\mathrm{h}}$ describing the Laplace pressure due to the curvature $\kappa_{\mathrm{h}}$. The constant $k_{0}$ describes the curvature at the top of the Leidenfrost drop [20] and is related to the size of the drop.

\subsubsection{Drop impact dynamics}

The Leidenfrost temperature is found to be elevated when a drop approaches the plate with a downward velocity $U$. The strong deceleration of the drop upon impact can overcome the stabilizing surface tension and deform the film, resulting in contact with the plate. The following section will present an overview of the dynamics of drop impact on unheated plates.

\section{Impact on unheated plates}

Prior to impact on a unheated plate, the drop already experiences the presence of the plate. The drop forces air to move ahead of it. When the distance to the wall diminishes, pressure builds up due to the viscosity of the air, whose magnitude becomes large enough to decelerate the centre of the drop, deforming the drop interface. As a result, drops generally touch down off-centre, and an air bubble is entrapped in the process [29-31].

The pressure field in the drop can be well described using Wagner's analogy [32-34]. Even though it is based on potential flow and does not take the air film into account, 
recent studies found good agreement were the Navier-Stokes equation were solved numerically for both the liquid and the air [35]. Of great importance is the occurrence of a localized pressure peak, resulting in the aforementioned off-centre touch down of the drop. The typical order of magnitude of this peak can be forty times higher than expected from the dynamic pressure of the drop: $p \approx \rho_{1} U^{2}$.

Once contact has established, the wetted area starts to grow, depending on the Weber number ( $W e=2 \rho U^{2} R / \gamma$, where $R$ is the drop radius) [36-39]. For $W e<1$ the wetting radius $R_{\mathrm{W}}$ grows due to capillary action, while for $W e>1$ the inertia of drops forces the spreading. If $W e$ is sufficiently high, splashing is observed: a lamella is ejected from the drop, which breaks up, releasing small satellite drops in radial direction [40-43]. A threshold for the impact velocity is obtained where splashing is found. This threshold is based on the velocity difference between the (decelerating) wetting radius $R_{\mathrm{W}}$ and the flow velocity in the drop away from the plate [44].

\section{Extension to heating}

In the present study we study drops impacting heated plates. The dynamics of the film should therefore be extended by the source term $j$, as we have seen in Equation 1.2. This source term, scaling inversely with gap thickness, becomes stronger the closer the film gets with the plate. The high pressure due to the impact can elevate the local saturation temperature, reducing the local superheat.

In the case the drop has made contact with the plate, the contact line dynamics will be strongly affected by the hot plate, since strong evaporation is expected here [45]. The local heating of the contact line will induce Marangoni flows in the drop as a result of the temperature difference with the remainder of the drop, which is still at ambient temperature. In general, the wetting radius can become a strong function of the plate temperature: $R_{\mathrm{W}}(t)=R_{\mathrm{W}}(T, t)$.

\subsubsection{Break down of framework}

We have seen that many phenomena can be well described by the continuum model. Two limitations are important to take into consideration: First, a result of the lubrication approximation, for $\Delta T \rightarrow 0$, still a finite film thickness is expected: For a film of constant thickness $h$, one obtains $h \propto \Delta T^{1 / 4}$ [17]. In this model, $h$ remains finite when $\Delta T \rightarrow 0$, which is not found experimentally [15]. Secondly, even in the case of a high plate temperature, the continuum model does not take the long range interaction between the liquid and the plate into account, with a typical interaction range of $10 \mathrm{~nm}$. Furthermore, since the length scale becomes of the order of the mean-free path of the vapour molecules, Knudsen effects are to be expected: the gas viscosity diminishes, while the heat transfer is enhanced by direct ballistic transport $[21,46-48]$.

\subsection{Guide to the thesis}

Since the liquid-solid interface is of such great importance in the heat transfer between a liquid and a solid, this thesis will commence by developing the experimental details related to frustrated total internal reflection (FTIR) (chapter 2). This technique has 


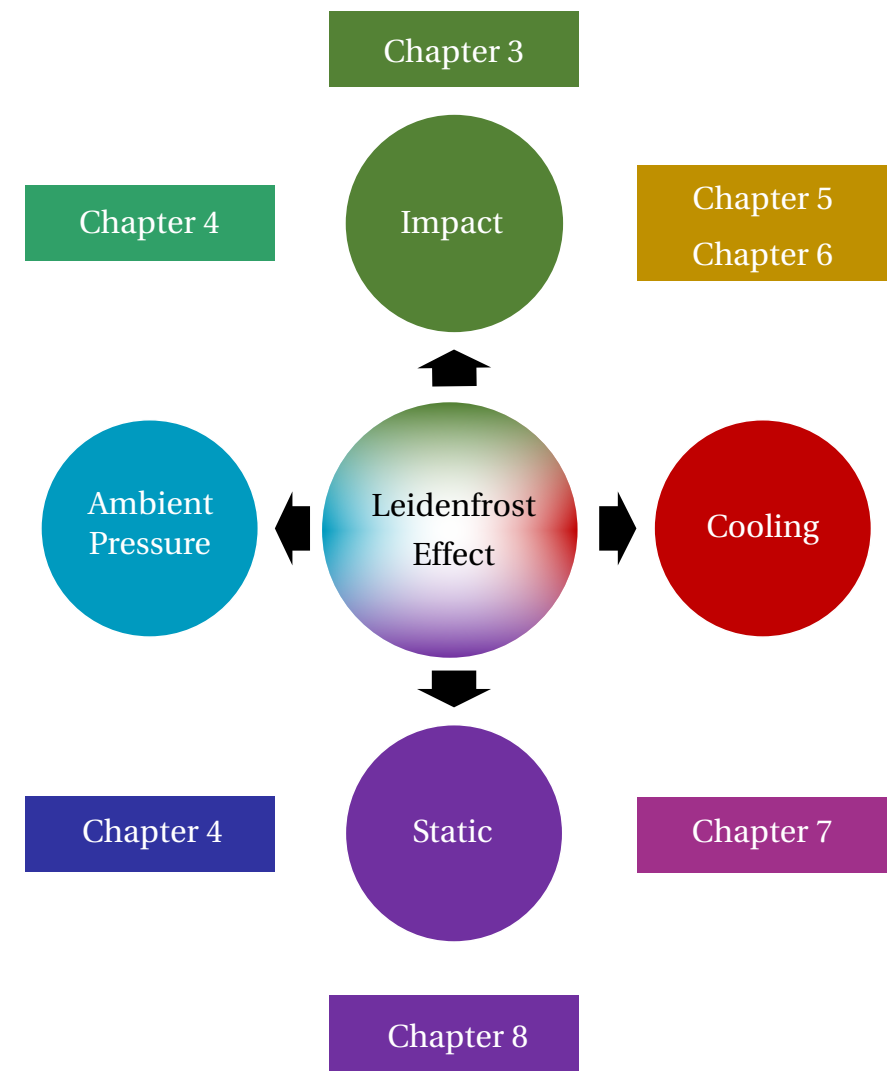

Figure 1.4: Overview of the main topics of the thesis.

proven to be extremely powerful for many experiments described in this thesis and is employed to measure thin air/vapour films between two media of high reflective index. In addition, FTIR also reveals the development of wetted areas, crucial to discriminate between Leidenfrost drops and those which make contact with the plate.

The following chapters are presented in the schematic of Fig. 1.4 and cover studies on the Leidenfrost effect. The chapters can be divided in four major topics: impact studies, static drops, ambient pressure influences and systems where cooling plays an important role.

Impact of drop on isothermal smooth surfaces are studied in chapter 3, revealing similarities and differences with drop impact on unheated plates. Three different boiling behaviours are characterized by comparing the spreading radius of the drop with the radius of the contact between the plate and drop. We refer to contact boiling when these two radii both represent the contact line of the drop. Increasing the plate temperature results at some point in a reduction of the contact line velocity and a lamella is ejected. Since there is still (a temporary) contact with the plate, we classify this as transition boiling. When no contact area is found at any time during the impact process we identify 
this as Leidenfrost boiling.

In chapter 4 we investigate how the boundaries between contact- transition and Leidenfrost boiling regimes change when varying the pressure: while the dynamic Leidenfrost temperature hardly changes, the boundary between contact and transition boiling remains strongly correlated with the static Leidenfrost temperature which is found to change with pressure. This observation disentangles the dominant physics at play in the various regimes: the boundary between contact and transition boiling is a result of the contact line dynamics, while the transition to Leidenfrost is dominated by the short time and length-scales of the impact dynamics.

The Leidenfrost effect is frequently encountered in the context of spray cooling, where the evaporation of drops cool the plate. In chapter 5 we study under what condition a plate is cooled down significantly and when it can be treated as isothermal. We compare the residence time of the drop near the plate with the typical thermal time scale. The latter depends strongly on the thermal properties of the plate.

An second study on cooling is described in chapter 6 . Here the impact of water drops are investigated. The drops generated a vertical spray while spreading, a signature for cooling effects. This is surprising as the experiments were performed on plates which were up to now considered to remain isothermal.

Chapter 7 focusses on how cooling plays a role in the static Leidenfrost effect. By the use of interferometry we study the temperature in a glass plate subject to the cooling by an evaporating Leidenfrost drop. With the aid of a numerical model we gain insight how the cooling affects the evaporation or the drop. This also allows us to extend existing Leidenfrost models for isothermal plates to plates which cool down by the evaporating drops.

An analytical study of 'boules' is presented in chapter 8. These are large Leidenfrost drops on a liquid bath. The deformability of the superheated bath completely changes the dominant forces in the vapour gap compared to Leidenfrost drops on a rigid plate. As a consequence, different scaling laws can be found for the thickness of the gap and evaporation rate of the drop. The last chapter summarizes the results presented in this thesis and provide an outlook for further studies. 


\section{Chapter 2}

\section{Measuring thin films using Quantitative Frustrated Total Internal Reflection ${ }^{\dagger}$}

In the study of interactions between liquids and solids, an accurate measurement of the film thickness between the two media is essential to study the dynamics. As interferometry is restricted by the wavelength of the light source used, recent studies of thinner films have prompted the use of frustrated total internal reflection (FTIR). In many studies the assumption of a simple exponential decay of the intensity with film thickness was used. In the present study we highlight that this model does not satisfy the Fresnel equations and thus gives an underestimation of the films. We show that the multiple reflections and transmissions at both the upper and the lower interfaces of the film must be taken into account to accurately describe the measured intensity. In order to quantitatively validate the FTIR technique, we measured the film thickness of the air gap between a convex lens of known geometry and a flat surface and obtain excellent agreement. Furthermore, we also found that we can accurately measure the elastic deformations of the lens under loads by comparing them with the results of Hertzian theory.

\footnotetext{
${ }^{\dagger}$ M. Shirota, M. A. J. van Limbeek, C. Sun and D. Lohse, submitted for publication
} 


\subsection{Introduction}

The interaction between liquids and solids has been of great interest lately in the study of the final phase before impact events. In many of these cases, a liquid is separated from a wall by a small gas or vapour film, which is drained or thickened through some dynamical process. These flows can typically be described by the lubrication approximation. A reliable quantitative measurement of the film thickness $d$ also allows to deduce the velocity in the film, which scales like $\propto d^{2}$ i.e., requires even extra precision.

Interferometry is commonly used for the measurement of thin film thicknesses. As the use of a single wavelength light source can only measure the absolute value of the gradient, often dual-wavelength interferometry is employed to obtain the shape of the thin film $[8,49]$. However, the exact film thickness is still unknown unless a reference thickness can be found. Colour interferometry using a white light source has successfully solved this problem $[30,50,51]$ : the multi-wavelength interference patterns contains the information of the absolute thickness, though the limited coherence length (typically a few mean wavelengths) renders the technique unusable for thicker films [52].

Films thinner than the wavelength of the light source however cannot be studied by interferometry. As a consequence, Kolinski, Rubinstein and co-workers [53-55] recently developed frustrated total internal reflection (FTIR) imaging for their study of thinner films. We have adopted this powerful technique $[45,56,57]$. Here, a transparent wall is illuminated at an angle larger than the critical one. In this situation an evanescent wave emerges in the thin film, whose intensity decays in an exponential-like manner within one wavelength distance from the wall. When a medium comes into the evanescent light, a portion of energy of the light transmits through the object which results in the decrease in the intensity of the reflection light. The energy loss of the light beam is thus a measure for the distance between the wall and the other medium. Further applications of this technique other than the film thickness measurement include particle image velocimetry in a near-wall flow [58], studies of moving contact lines and wetting/drying behaviour of heated surfaces in nucleate boiling studies [13, 45, 56, 57].

The focus of this paper is to discuss the FTIR technique, the post-processing of the data and to explain and validate the analysis to obtain height profiles from the measured intensity. Previous models [53-56] neglect multiple transmissions and reflections at both top and bottom interfaces of the thin film and also the light polarization. Those assumptions imply that the measured evanescent light intensity decays exponentially with increasing film thickness. However, we observed deviations from such behaviour, which we can account for by taking the multiple reflections and the polarization into account.

\subsection{Experimental setup and image analysis}

\subsubsection{Setup}

A schematic overview of the essential parts of the setup is shown in Fig. 2.1. A 30 $\mathrm{mW}$ laser with a wavelength of $643 \mathrm{~nm}$ was used as a mono-chromatic light source to illuminate the top of the prism, which is the measurement location. As the polarization is important for a quantitative height measurement, the light beam was designed to 


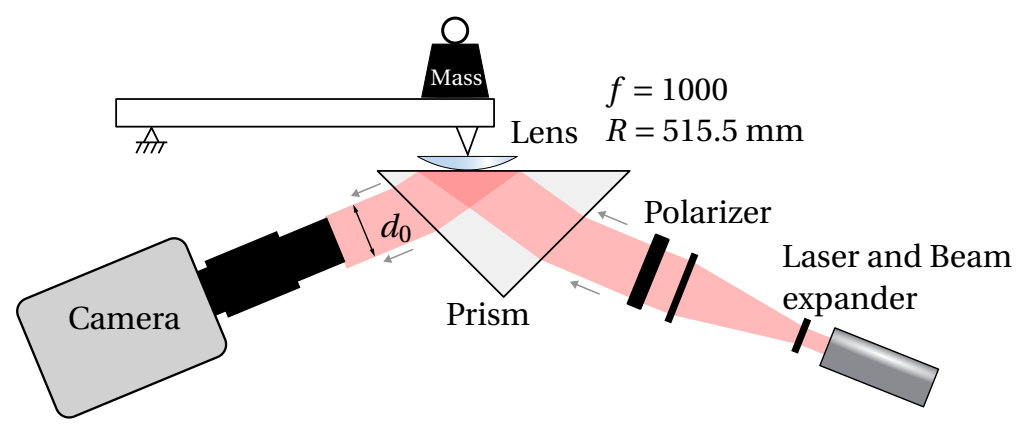

Figure 2.1: Schematic of a frustrated total internal reflection set up, which is calibrated by the use of forcing a lens onto the prism. The camera focusses on the top interface of the prism. The polariser is to control the polarization of the monochromatic light source, in this case a laser.

travel through a polariser to control the polarization. To obtain full illumination of the measurement area, we expanded the beam by a Keplerian expander consisting of two plano-convex lenses of proper combination. The reflected light intensity was then recorded by a high-speed camera at a recording rate of $60 \mathrm{fps}$ with the shutter speed set at $1 / 25000 \mathrm{~s}$ and a spatial resolutions of $1280 \times 1024$ pixel. The camera sensor had 8-bit intensity depth and a linear sensitivity for the light intensity. A high-speed camera was used instead of a still camera in order to analyse and reduce the time variation of the background intensity caused by both the fluctuation of the laser output and of thermal fluctuation of the camera sensor. The camera was equipped with a longdistance microscope focussed on the top interface of the prism to achieve a spatial resolution of typically $18 \mu \mathrm{m} /$ pixel.

The two basic requirements for FTIR to work are first that the prism's material must be transparent for the light wavelength, as the event is imaged through the solid itself; secondly, the surface is required to be optically flat, i.e. not curved, and its roughness must be smaller than the wavelength. A good example is a right-angle prism, which we used in our case. When the angle of incidence on the top surface of the prism $\phi$ exceeds the critical angle, the light is totally reflected and exits the prism via the side face of the prism. Therefore, we require the refractive indices of the prism $n_{1}$ and the third medium $n_{3}$ to be larger than that of the second medium $n_{2}$, i.e. $n_{1}, n_{3}>n_{2}$.

In our experiment, we verified the FTIR technique by measuring the thin air film between the flat surface of the prism and the curved surfaces of a convex lens. The radius of curvature of the lens was $515.5 \mathrm{~mm}$ (focal length was $1000 \mathrm{~mm}$ ). Its interface was at about $1 \mu \mathrm{m}$ away at $1 \mathrm{~mm}$ distance from the center of the lens. We also measured the gaps under the deformed lens with loads ranging from $30 \mathrm{~g}$ to $190 \mathrm{~g}$. The loads resulted in the increase of the flat area, or the contact radius, at the center of the lens.

\subsubsection{Image calibration and analysis}

The image analysis consists of three main steps: (1) image transformation, (2) intensity normalization, and, in the present case of axisymmetry, (3) calculation of the averaged 
(a)

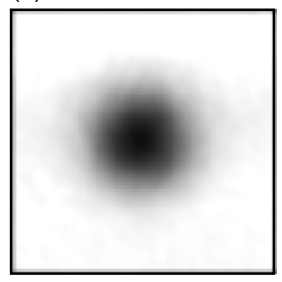

(c)

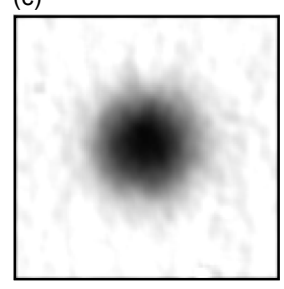

(b)

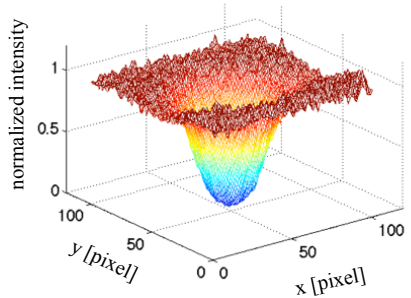

(d)

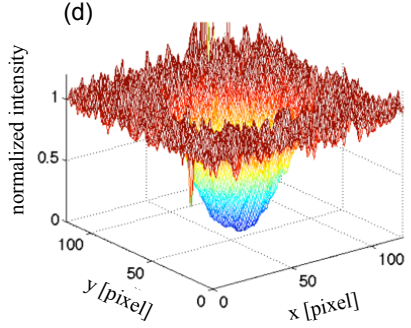

Figure 2.2: Typical example of the FTIR image for the air gap between a convex lens ( $\mathrm{f}=$ $1000 \mathrm{~mm}$ ) and a plane surface. Normalized intensity profile of s-polarized (a, b) and p-polarized (c, d) laser light.

radial intensity profile. The image transformation is to compensate for the optical transformation by the setup. Depositing a circular drop on the top prism face results in a black spot on the camera, since the drop changes the refractive index and hence light can exit the prism. However, the spot appears elliptical, with same ratio between the major and minor axis, $D_{\mathrm{L}}$ and $D_{\mathrm{S}}$, respectively, which is used for the back transformation of the image. This ratio is related to the angle of incidence $\phi_{1}$, and the ratio in refractive index $n=n_{2} / n_{1}$ see Fig. 2.3:

$$
\frac{D_{\mathrm{S}}}{D_{\mathrm{L}}}=\frac{\cos \phi_{1}}{\cos \left(\phi_{1}-45\right)} \times \cos \left(\sin ^{-1}\left(n \sin \left(\phi_{1}-45\right)\right)\right) \equiv F\left(\phi_{1}\right),
$$

which is of great importance for the quantitative height measurement of the thin films.

The transformed image was then divided by the background image. Typical examples of the normalized image are shown in Fig. 2.2 for a s and p-polarized laser. Note here that the image for $\mathrm{p}$-polarized laser shows a more broadened profile than the s-polarized one, since the reflection intensity for p-polarized light increases more gradually in general than s-polarized one with increasing air film thickness. Let us stress that the polarization of the incident light does affect the reflection intensity even with the evanescent light.

Since we use an axisymmetric object for our calibration, the image was sampled by 100 different angles from the objects centre, to obtain an averaged intensity profile in radial direction. The centre was found by fitting a three dimensional quadratic surface to the intensity profile, see section 2.A. 


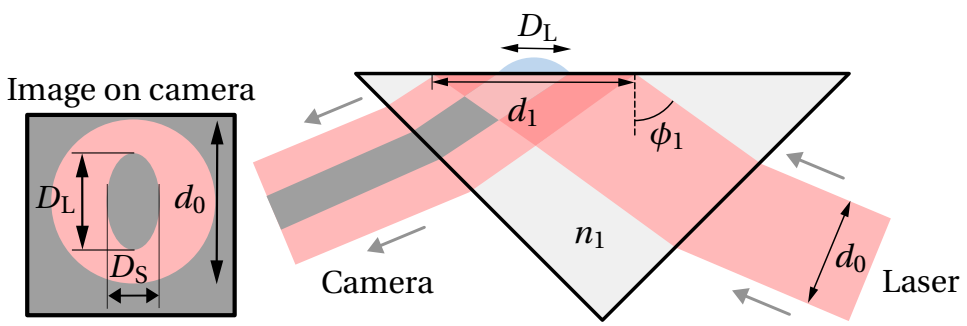

Figure 2.3: Schematic diagram of the optical path a laser beam of with $d_{0}$ follows and the image on the as recorded by the camera. A spherical drop of diameter $D_{\mathrm{L}}$ is placed to light to escape the prism. The ratio between $D_{\mathrm{L}}$ and $D_{\mathrm{S}}$ is used to measure the angle of incidence $\phi_{1}$ using Equation 2.1. $n_{1}$ is the prism refractive index and $d_{1}$ the projected laser beam.

\subsubsection{Operating conditions}

Next to the condition $n_{1}, n_{3}>n_{2}$ for the FTIR technique to work, the angle of incidence $\phi_{1}$ is to be beyond the critical angle, i.e. $\phi_{1}>\sin ^{-1}\left(n_{2} / n_{1}\right)$. A similar criterion arises from the fact that light should be able to propagate in the drop: $\phi_{1}<\sin ^{-1}\left(n_{3} / n_{1}\right)$. Figure 2.4 shows Equation 2.1 for $n=1.5$ and 1.75, representing a glass and sapphire prism, respectively, where the proper operating range is indicated in blue together with the region where $\phi_{1}<\sin ^{-1}\left(n_{3} / n_{1}\right)$ in red. From this relation, $\phi_{1}$ can be obtained using the measured aspect ratio $D_{\mathrm{S}} / D_{\mathrm{L}}$. It should be noted that for increasing $n_{1}$, at some point the operating range becomes multivalued. In this case, to obtain the correct $\phi_{1}$, the angle of incidence of the laser beam with the side wall of the prism is to be measured experimentally.

\subsection{Principle of quantitative height measurement with FTIR}

In the following we will discuss more precisely the relation between the reflected light intensity and the experimental parameters, and derive the formula for the frustrated total internal reflection based on thin film theory. We follow the classical study by Court et al. [59] (see also the review paper by Zhu et al. [60]).

\subsubsection{When the angle of incidence is smaller than the critical angle}

Let us first consider an interface illuminated by a light source with the angle of incidence $\phi_{1}$ being less than the critical angle. A part of the light energy reflects and the other part transmits across the interface, depending on the materials' reflective indices. If there are three media, i.e., two interfaces, multiple transmissions and reflections will occur.

A schematic of this configuration is shown in Fig. 2.5, where the second medium is of thickness $d$. The difference in optical path length, or the phase difference among the reflected lights, results in constructive and destructive interferences. The phase 

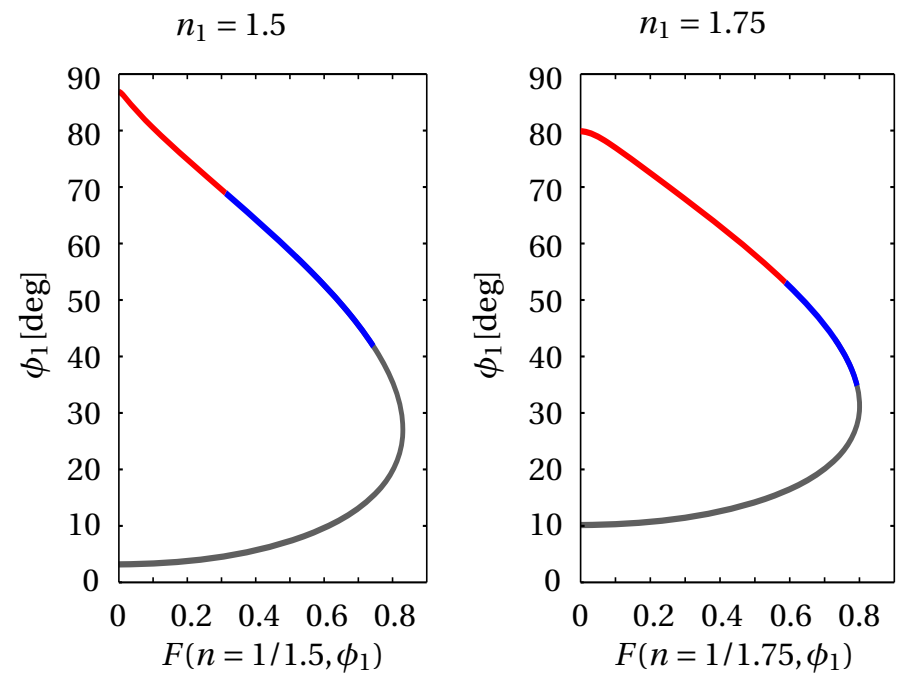

Figure 2.4: Solutions of the optical transfer function $F$ (Equation 2.1), used to obtain the angle of incidence $\phi_{1}$ for two prism materials. Increasing the refractive index ratio $n=n_{2} / n_{1}$ shows a shift towards lower angles in the operating range (blue). The red curves indicate angles for which direct contact between the third medium (here $n_{3}=1.4$ ) and the prism also exhibits total internal reflection and hence the method becomes dysfunctional.

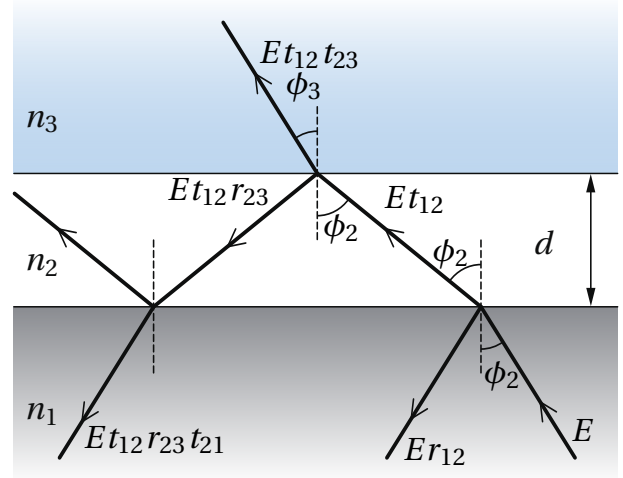

Figure 2.5: Close-up of the transmitted and reflected light at a thin homogeneous film of low refractive index. 
difference can be expressed by multiplying the reflected wave amplitudes by $e^{j \delta}$, where $j$ is the imaginary unit and $\delta$ the phase difference between successive reflected waves. The phase difference between consecutive reflected lights from the optical path difference is given by:

$$
\begin{aligned}
\Delta l & =n_{2} l_{2}-n_{1} l_{1} \\
& =\frac{2 d n_{2}}{\cos \phi_{2}}-\frac{2 d n_{2} \sin ^{2} \phi_{2}}{\cos \phi_{2}}=2 d n_{2} \cos \phi_{2} .
\end{aligned}
$$

Hence the change in phase $\delta=2 \pi \Delta l / \lambda$ can be expressed using Snell's law as

$$
\delta=\frac{4 \pi d}{\lambda} \sqrt{n_{2}^{2}-n_{1}^{2} \sin ^{2} \phi_{1}}
$$

At every interface, a part of the light energy is reflected and the other part is transmitted. The Stokes' relations connect the transmission and reflection coefficients, $t_{12}$ and $r_{12}$, for light originating in the $n_{1}$ medium and the coefficients $t_{21}$ and $r_{21}$ for light originating in the $n_{2}$ medium:

$$
t_{12} t_{21}+r_{12}^{2}=1, \quad r_{12}=-r_{21}, \quad r_{23}=-r_{32} .
$$

The reflected wave amplitudes of the consecutive lights from the $(1,2)$ interface can be calculated using the incident amplitude $E^{i}$ : they are $r_{12} E^{i}, t_{12} t_{21} r_{23} E^{i} e^{j \delta}$, $t_{12} t_{21} r_{21} r_{23}^{2} E^{i} e^{2 j \delta}, \ldots$ where we have defined $r_{23}$ as the amplitude reflection coefficient for the light incident on the $(2,3)$ interface from medium 2 . Adding these multiple reflection contributions yields the following geometric series, while making use of the Stokes' relations Eq. (2.4) to obtain the total reflected amplitude $E^{r} / E^{i} \equiv r$ :

$$
\begin{aligned}
\frac{E^{r}}{E^{i}}=r & =r_{12}+\frac{t_{12} t_{21} r_{23} e^{j \delta}}{1-r_{21} r_{23} e^{j \delta}} \\
& =r_{12}+\frac{\left(1-r_{12}^{2}\right) r_{23} e^{j \delta}}{1+r_{12} r_{23} e^{j \delta}} \\
& =\frac{r_{12}+r_{23} e^{j \delta}}{1+r_{12} r_{23} e^{j \delta}}
\end{aligned}
$$

For polarized light, the Stokes' relations are described by the Fresnel equations, which depend on the polarization of the light. For p-polarized light, they are given as follows:

$$
\begin{aligned}
& r_{12, \mathrm{p}}=\left(E_{\mathrm{p}}^{r} / E_{\mathrm{p}}^{i}\right)_{12}=\frac{n_{2} \cos \phi_{1}-n_{1} \cos \phi_{2}}{n_{2} \cos \phi_{1}+n_{1} \cos \phi_{2}}, \\
& r_{32, \mathrm{p}}=\left(E_{\mathrm{p}}^{r} / E_{\mathrm{p}}^{i}\right)_{32}=\frac{n_{2} \cos \phi_{3}-n_{3} \cos \phi_{2}}{n_{2} \cos \phi_{3}+n_{3} \cos \phi_{2}} .
\end{aligned}
$$

Similarly, the s-components are

$$
\begin{aligned}
& r_{12, \mathrm{~s}}=\left(E_{\mathrm{s}}^{r} / E_{\mathrm{s}}^{i}\right)_{12}=\frac{n_{1} \cos \phi_{1}-n_{2} \cos \phi_{2}}{n_{1} \cos \phi_{1}+n_{2} \cos \phi_{2}}, \\
& r_{32, \mathrm{~s}}=\left(E_{\mathrm{s}}^{r} / E_{\mathrm{s}}^{i}\right)_{32}=\frac{n_{3} \cos \phi_{3}-n_{2} \cos \phi_{2}}{n_{3} \cos \phi_{3}+n_{2} \cos \phi_{2}} .
\end{aligned}
$$


The angles $\phi_{2}$ and $\phi_{3}$ can be expressed in terms of $\phi_{1}$ using Snell's law. The full analysis now becomes a sole function of the angle of incidence $\phi_{1}$ and the refractive indices of the three media.

\subsubsection{When the angle of incidence is larger than the critical angle}

Let us now treat specifically the case of FTIR. Then, $\delta=(4 \pi d / \lambda)\left(n_{2}^{2}-n_{1}^{2} \sin ^{2} \phi_{1}\right)^{1 / 2}$ becomes imaginary, since $n_{1} \sin \phi_{1}>n_{2}$, i.e., light is incident at an angle that is greater than the critical angle $\phi_{\mathrm{c}}$. Now the contribution from the phase difference $e^{j \delta}$ becomes an exponentially decaying function $e^{-\delta^{\prime}}$, where $\delta^{\prime}=(4 \pi d / \lambda)\left(n_{1}^{2} \sin ^{2} \phi_{1}-n_{2}^{2}\right)^{1 / 2}$ is real. Furthermore, $\phi_{2}$ also becomes a complex quantity with a real part of $90^{\circ}$. Consequently, the reflection coefficient $r_{\mathrm{ij}}$ becomes complex as well.

The absolute values of these complex coefficients are $\left|r_{\mathrm{ij}}\right|=1$ describing the total internal reflection. One can also calculate the phase difference between these reflected waves and the incidence wave by $\tan ^{-1}\left(\operatorname{imag}\left(r_{\mathrm{ij}}\right) / \operatorname{real}\left(r_{\mathrm{ij}}\right)\right)$. By substituting the Fresnel equations (Eqs. (2.6) to (2.9)) into Eq. (2.5), we can obtain the total reflection amplitude for both the p- and s-component of the light. In summary, the reflectivity can be obtained by a single expression, given by Eq. (2.5), regardless of whether $\phi_{1}$ is smaller or larger than $\phi_{\mathrm{c}}$.

Let us stress that the preceding analysis shows that, even with an evanescent wave, the Fresnel equations should be satisfied at the interfaces. In Kolinski et al.'s analysis [5355], it is assumed that all of the evanescent light's energy at height $d$ is transmitted into the third medium. In this way one neglects the polarization dependence as described by the Fresnel equations, resulting in a deviation from the correct result under some conditions, as we will show in the following section.

\subsection{Results and discussion}

\subsubsection{Reflectance by FTIR}

The analysis is evaluated for our setup (see Fig. 2.1) and is shown in Fig. 2.6. For comparison, the model proposed by Kolinski et al. [53-55] (K-model) is also shown with the decay length $\beta=\frac{\lambda n}{4 \pi}\left(\sin ^{2} \phi_{1}-n^{2}\right)^{-1 / 2}$, which describes total internal reflection at the interface in the absence of a third medium. Here, $n$ represents the ratio of refractive indices, $n_{2} / n_{1}$. As shown in this figure, the intensity with p-polarized light increases more gradually than the s-polarized one with increasing film thickness. It is also clearly shown that the K-model always underestimates the film thickness for a given intensity.

\subsubsection{Validation and uncertainty analysis with a lens profile}

Let us compare the FTIR model calculation with the profile of the convex lens. The presented analysis is prone to error by uncertainties in the angle of incidence $\phi_{1}$, the wave length of the light source $\lambda$, and the three refractive indices $n_{1}, n_{2}$ and $n_{3}$. In order to estimate the errors of the analysis, the influence of all uncertainties on the intensityheight curve are investigated numerically, as shown in Fig. 2.7. Two bounds can be constructed with the maximum deviation from the intensity-height curve, representing 


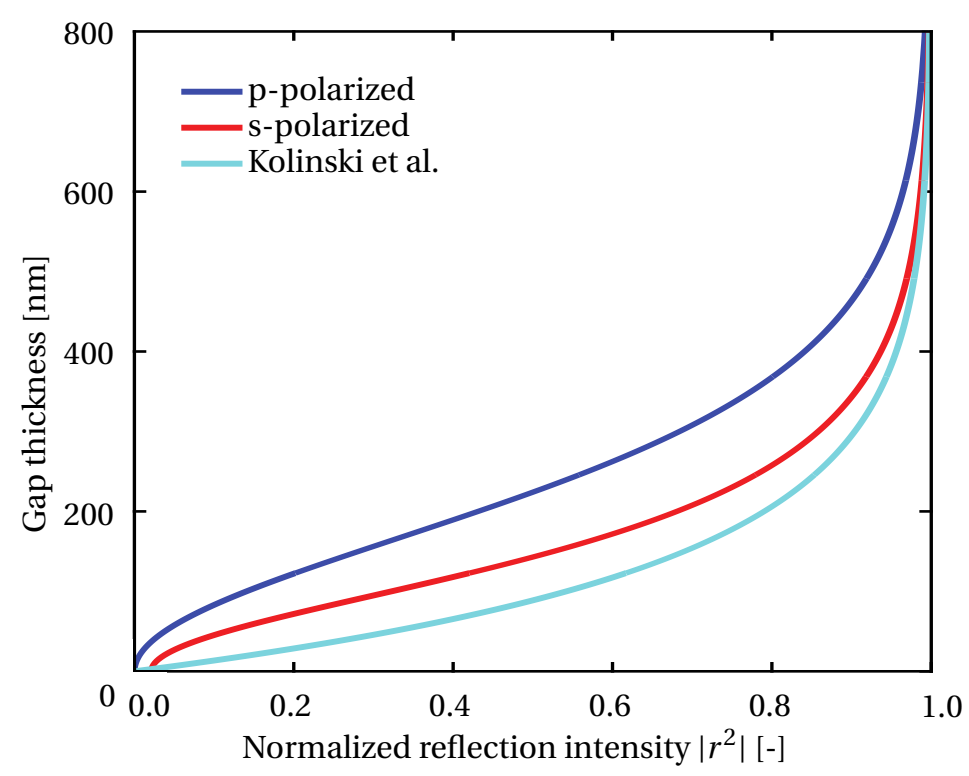

Figure 2.6: Normalized reflection intensity, $|r|^{2}$, as a function of film thickness. $n_{1}=$ 1.765 (sapphire), $n_{2}=1$ (air/vapor), and $n_{3}=1.5$ (glass). The angle of incidence is $\phi_{1}=$ $37.6^{\circ}$. For comparison, the result of the model of Kolinski et al. [53-55] is also shown (lower light blue curve).

the confidence bounds of the analysis: the upper (dashed) and lower (dotted) bounds for both p- and s-polarized light. Next to these uncertainties, the technique is subject to fluctuations in the measured intensity and the total error of the measurements can then be plotted. These fluctuations arise from a finite bit-depth of the camera, thermal fluctuations of the sensor and temporal fluctuation in the laser intensity. In the present case, we measured these to be smaller than $2 \%$ which can result in bias errors. For high intensity, the curves approach the asymptote of infinite film thickness and hence measurements become unreliable.

Figure 2.8 shows the comparison between the measurements and the lens profile. Here, the measurement data were shifted to the lower by $19 \mathrm{~nm}$ and $44 \mathrm{~nm}$ for $\mathrm{p}$ - and s-polarized light, respectively, to fit the lens profile at the gap thickness $\approx 0.1 \mu \mathrm{m}$. The FTIR model shows excellent agreement with the theoretical lens profile in terms of the curvature, which is clearly shown in the semi-log plot. The deviations at both the lower and the higher gap thicknesses arise mainly from the non-linearity of the sensor sensitivity at the extreme intensities. The overall offset is found to be the order of the fluctuations described above, i.e., the temporal fluctuation in the laser intensity, the roughness of the lens and expected surface contaminations. It is also shown that the reconstructed profile by K-model with the average intensity of both p- and s-polarized light does not well describe the curvature of the lens. 


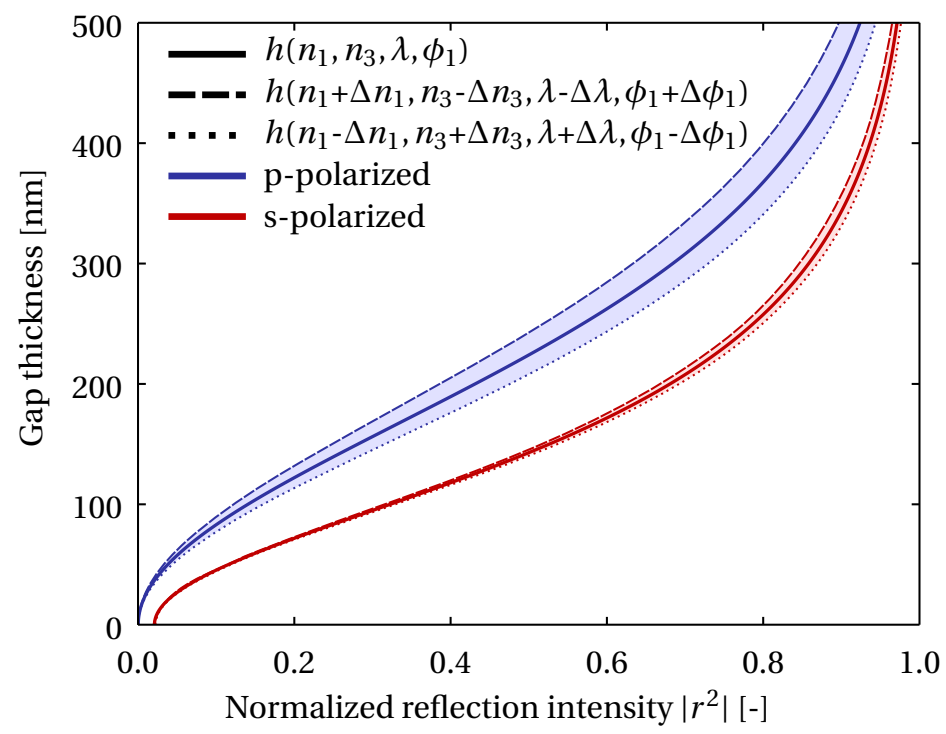

Figure 2.7: Calculated confidence bounds and error analysis for a glass lens ( $n=$ $1.510 \pm 0.001)$ on a sapphire prism $(n=1.765 \pm 0.001)$. Every uncertainty is either added or subtracted to maximize the deviation. Data is shown for red laser light $(\lambda=(643.0 \pm 0.5) \mathrm{nm})$ at an angle of incidence of $(38.0 \pm 0.5)^{\circ}$.

\subsubsection{Validation with the elastic deformation of a lens by load}

Another validation of the FTIR technique was examined by comparing the elastic deformations of the lens with the Hertzian theory [61] of a contact between a sphere and a flat surface without any attractive forces between them.

We applied the external forces by putting weights ranging from 30 to $190 \mathrm{~g}$ on the lens. The Hertzian model predicts the contacting radius $a$ as follows:

$$
a=\left(\frac{R L}{K}\right)^{1 / 3} .
$$

Here $L$ represents the externally applied loading force, $K=4 / 3\left[\left(1-v_{1}^{2}\right) / E_{1}+(1-\right.$ $\left.\left.\left.v_{2}^{2}\right) / E_{2}\right)\right]^{-1}$ is the effective elastic modulus with $E_{1,2}$ and $v_{1,2}$ being elastic moduli and Poisson's ratios of sphere and plane, respectively. As shown in Fig. 2.9, the contacting radius increases with the load weight. With this contacting radius $a$ and the radius of curvature of the lens $R$, the lens profile $y$ can be shifted as follows:

$$
y= \begin{cases}0 & (r \leq a), \\ \sqrt{R^{2}-a^{2}}-\sqrt{R^{2}-r^{2}} & (r>a),\end{cases}
$$

where $r$ is the radial coordinate. The shifted profile with Hertzian model well predicts the contacting radius although it slightly overestimates the curvature. 


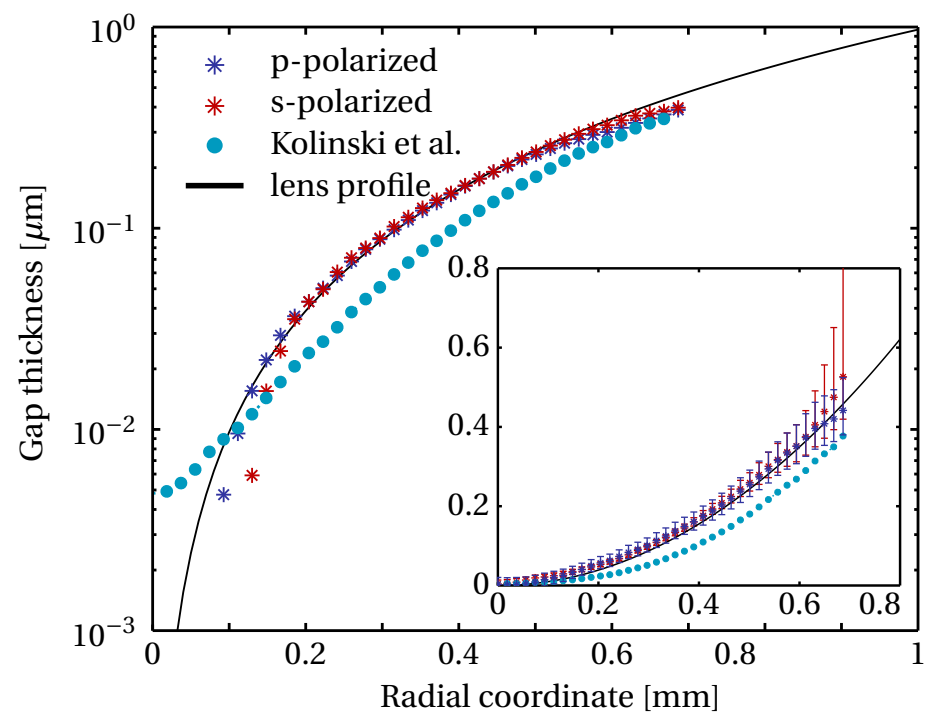

Figure 2.8: Measured film thickness of the air gap between a glass lens $(f=1000)$ and a sapphire prism without forcing. Both the s and p-polarized light show excellent agreement with the theoretical lens profile, while the K-model systematically underestimates it. The measurement data are shifted by $19 \mathrm{~nm}$ and $44 \mathrm{~nm}$ for $\mathrm{p}$ - and s-polarized light, respectively, to compensate for the bias error. For K-model, the average of both p- and s-polarized light intensities is used. Inset shows the same data but in linear-linear plot with the confidence bounds shown in Fig. 2.7.

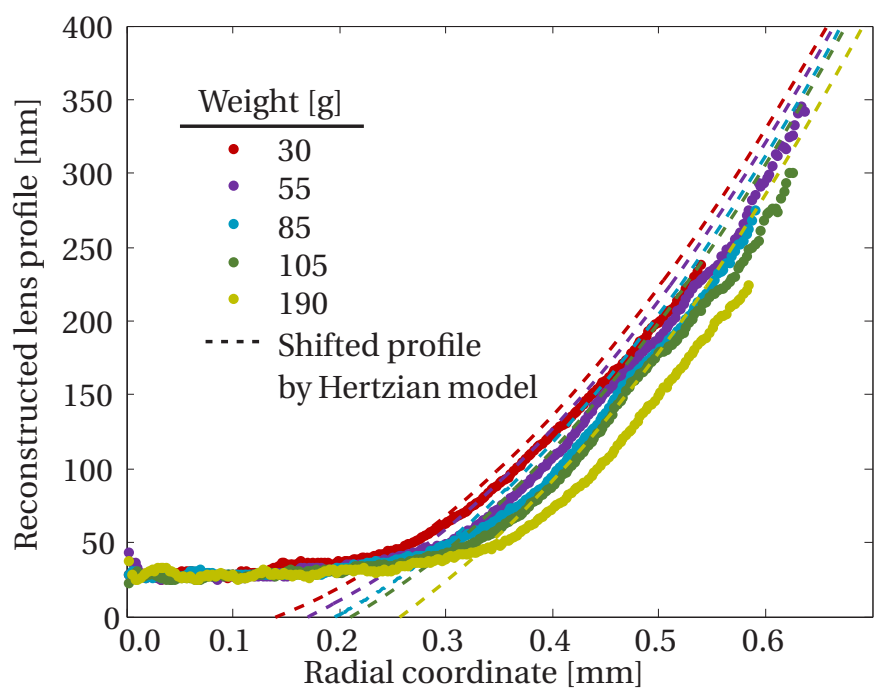

Figure 2.9: Elastic deformations of a $f=1000 \mathrm{~mm}$ convex-plano lens by externally applied forces. Data points of the various colors represent the measured data and the dashed lines of the corresponding colors the results of the Hertzian model. 


\subsection{Conclusion}

We conclude that the FTIR measurement is a reliable technique for a quantitative measurement of thin air layers between two substrates of higher refractive indices, with an accuracy of the order of $10 \mathrm{~nm}$. We have verified the applicability for quantitative thickness measurements of thin air films using frustrated total internal reflection (FTIR) technique, that was originally developed by Kolinski, Rubinstein and coworkers. We extended the analysis and revealed that the Fresnel relations at the top and bottom interfaces of the film should be taken into account, and as a result, that the polarization of the incident light plays an essential role. The successful measurement of the film between a lens and a plate showed good agreement with the analysis presented, proofing that a simple exponential decay of the intensity with film thickness as a model for the evanescent light without a third medium is too approximative. A detailed error analysis was provided to study the measurement reliability and showed that the error diverges only for films thicker than approximately one wave length of the light source.

\section{Acknowledgement}

We thank S.M. Rubinstein and J. Kolinski for helpful discussions. This work was partially supported by Fundamenteel Onderzoek der Materie and by an ERC-Advanced Grant. 


\section{A Step-by-step procedure of the image processing to obtain the gap thickness from an FTIR image}

This supplementary document describes how to obtain the film thickness from an image taken with frustrated total internal reflection (FTIR) imaging. The process consists of four main steps, for which we made use of the commercial software package Matlab [62].

\section{Step 1: Calculation of the angle of incidence $\phi_{1}$}

As explained in the section 2.2 in the main text, we calculate the angle of incidence $\phi_{1}$ from the aspect ratio of the elliptically deformed drop image. The summary of the image processing to obtain the aspect ratio is shown in Fig. A-1. The edge detection algorithm (using the canny method) was first applied. We then fitted the following symmetric function to the outline of the drop described in a polar coordinate with the origin being at the center of the drop:

$$
r(\theta)=R_{0}+R_{2} \cos ^{2}(\theta+\psi)+R_{4} \cos ^{4}(\theta+\psi),
$$

where $\theta$ is the angle measured from the horizontal axis, and $R_{0}, R_{2}, R_{4}$ and $\psi$ were determined by the Matlab function fit with the non-linear least square method. The aspect ratio was then determined in terms of $R_{0}, R_{2}$ and $R_{4}$ :

$$
\frac{D_{\mathrm{S}}}{D_{\mathrm{L}}}=\frac{R_{0}-R_{2}-R_{4}}{R_{0}+R_{2}+R_{4}}
$$

\section{Step 2: Calculation of the transformation function $F\left(\phi_{1}\right)$ and evaluation of $\phi_{1}$}

For a given relative refractive index of the medium 1 to the medium 2, i.e. $n=n_{1} / n_{2}$, we can calculate the transformation function $F$ as a function of the angle of incidence $\phi_{1}$ by using Eq. (1) in the main text:

$$
\frac{D_{\mathrm{S}}}{D_{\mathrm{L}}}=\frac{\cos \phi_{1}}{\cos \left(\phi_{1}-45\right)} \times \cos \left(\sin ^{-1}\left(n \sin \left(\phi_{1}-45\right)\right)\right) \equiv F\left(\phi_{1}\right) .
$$

Note that the function $F$ coincides with the aspect ratio of the drop. If we plot $\phi_{1}$ as a function of $F$, then we obtain the relation as shown in Fig. 2.4, from which we can determine $\phi_{1}$ for a given aspect ratio calculated in the previous step.

\section{Step 3: Calculation of the reflectance $|r|^{2}$}

The intensity of the reflection wave, the so-called reflectance which is determined as the square of the absolute value of the complex reflection amplitude, $|r|^{2}$, can be calculated using Eq. (5) in the main text combined with the Fresnel equations Eqs. (6) and (7) for p-polarized light or Eqs. (8) and (9) for s-polarized light. Note that $r_{12}$ and $r_{23}$ become complex quantities, so does $r$, when the angle of incidence exceeds the critical angle. 
Hence we obtain for the p-component:

$$
\begin{aligned}
r_{12, \mathrm{p}}= & \frac{n_{2} \cos \phi_{1}-n_{1} \sqrt{1-\left(\frac{n_{1}}{n_{2}} \sin \phi_{1}\right)^{2}}}{n_{2} \cos \phi_{1}+n_{1} \sqrt{1-\left(\frac{n_{1}}{n_{2}} \sin \phi_{1}\right)^{2}}} \\
r_{32, \mathrm{p}}= & \frac{n_{2} \sqrt{1-\left(\frac{n_{1}}{n_{3}} \sin \phi_{1}\right)^{2}}-n_{3} \sqrt{1-\left(\frac{n_{1}}{n_{2}} \sin \phi_{1}\right)^{2}}}{n_{2} \sqrt{1-\left(\frac{n_{1}}{n_{3}} \sin \phi_{1}\right)^{2}}+n_{3} \sqrt{1-\left(\frac{n_{1}}{n_{2}} \sin \phi_{1}\right)^{2}}} .
\end{aligned}
$$

Similarly, the s-components are

$$
\begin{aligned}
r_{12, \mathrm{~s}}= & \frac{n_{1} \cos \phi_{1}-n_{2} \sqrt{1-\left(\frac{n_{1}}{n_{2}} \sin \phi_{1}\right)^{2}}}{n_{1} \cos \phi_{1}+n_{2} \sqrt{1-\left(\frac{n_{1}}{n_{2}} \sin \phi_{1}\right)^{2}}} \\
r_{32, \mathrm{~s}}= & \frac{n_{3} \sqrt{1-\left(\frac{n_{1}}{n_{3}} \sin \phi_{1}\right)^{2}}-n_{2} \sqrt{1-\left(\frac{n_{1}}{n_{2}} \sin \phi_{1}\right)^{2}}}{n_{3} \sqrt{1-\left(\frac{n_{1}}{n_{3}} \sin \phi_{1}\right)^{2}}+n_{2} \sqrt{1-\left(\frac{n_{1}}{n_{2}} \sin \phi_{1}\right)^{2}}},
\end{aligned}
$$

where we used the identity $\cos \left(\sin ^{-1}(x)\right)=\sqrt{1-x^{2}}$. Note that $r_{12}$ and $r_{23}$ become complex quantities, when the angle of incidence exceeds the critical angle. They are two sets of geometric coefficients used in:

$$
|r|^{2}=\left|\frac{r_{12}+r_{23} e^{j \delta}}{1+r_{12} r_{23} e^{j \delta}}\right|^{2},
$$

depending on the polarization of the light.

Since the result is not invertible to solve for the gap separation, a lookup table is generated: $\delta=4 \pi d\left(n_{2}^{2}-n_{1}^{2} \sin ^{2} \phi_{1}\right)^{1 / 2} / \lambda$ for the gap thickness $d$ ranging from 0 to approximately one wavelength. One can numerically calculate the reflectance as a function of the gap thickness. An example of the result is shown in Fig. 6, where the gap thickness is plotted against the reflectance. $|r|^{2}$ is then used as a lookup table to convert the measured intensities into the height profile.

\section{Step 4: Evaluation of the averaged intensity for the lens profile}

If the film has an axisymmetric profile, for example the one between a flat solid and a convex lens as examined in the present study, the intensity averaging provides more precise results. In advance of the averaging, the center of the axisymmetric film must be determined. This is easily achieved by fitting a three dimensional quadratic surface to the intensity profile. An example of the surface fitting is shown in Fig. 2.11. From the center, the intensity profiles in radial direction for 100 different angles were extracted using the Matlab function improfile. Finally, the averaged intensity profile was obtained by taking the average of the 100 profiles. 

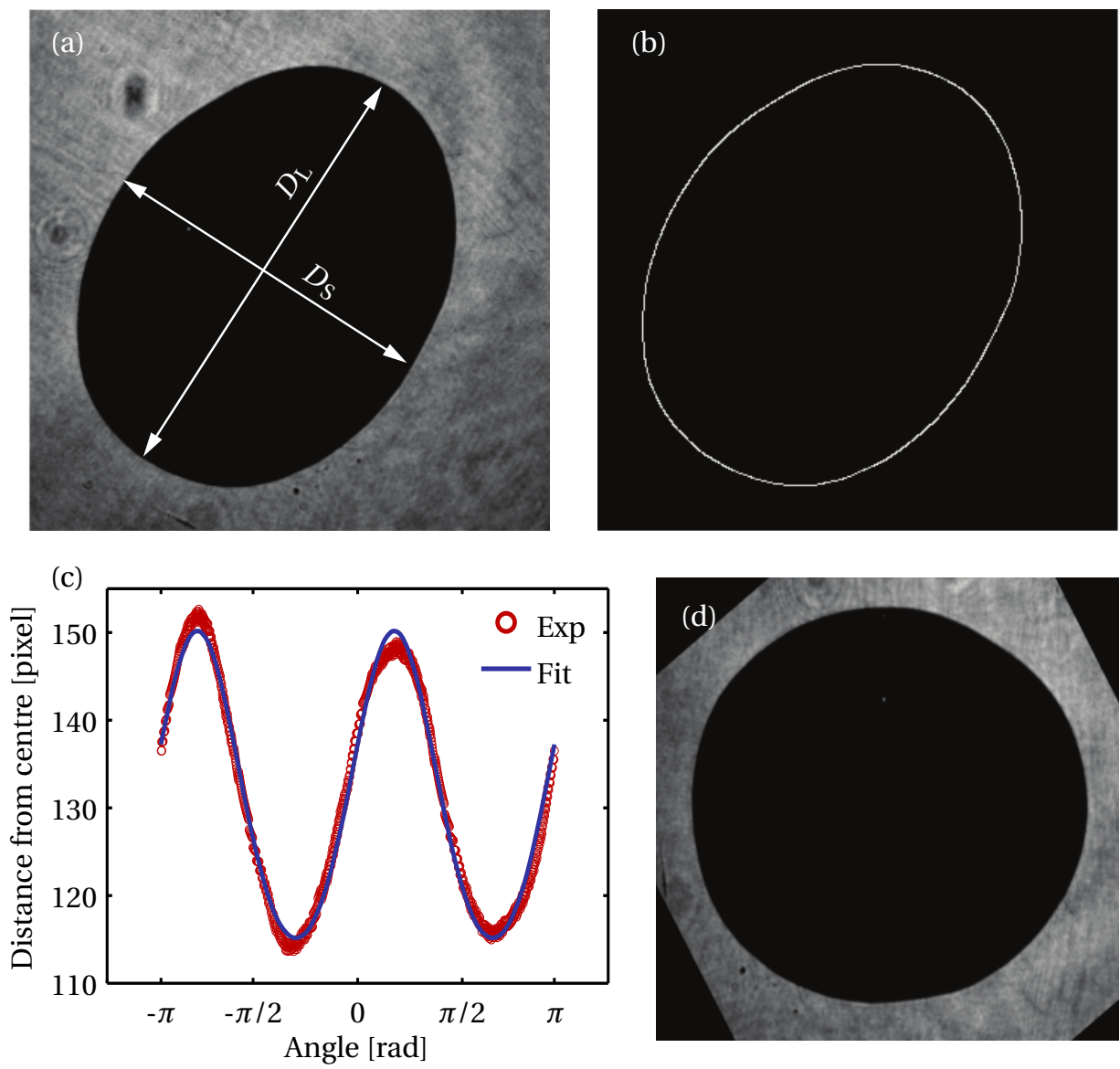

Figure 2.10: Image processing to obtain the aspect ratio of the drop. (a) original image, (b) the outline of the original image, (c) distance from the center to the outline of the drop in a polar coordinate with a fitted curve in red line, (d) transformed image after applying the backward transformation using the aspect ratio and the rotation angle obtained in the process (c). 


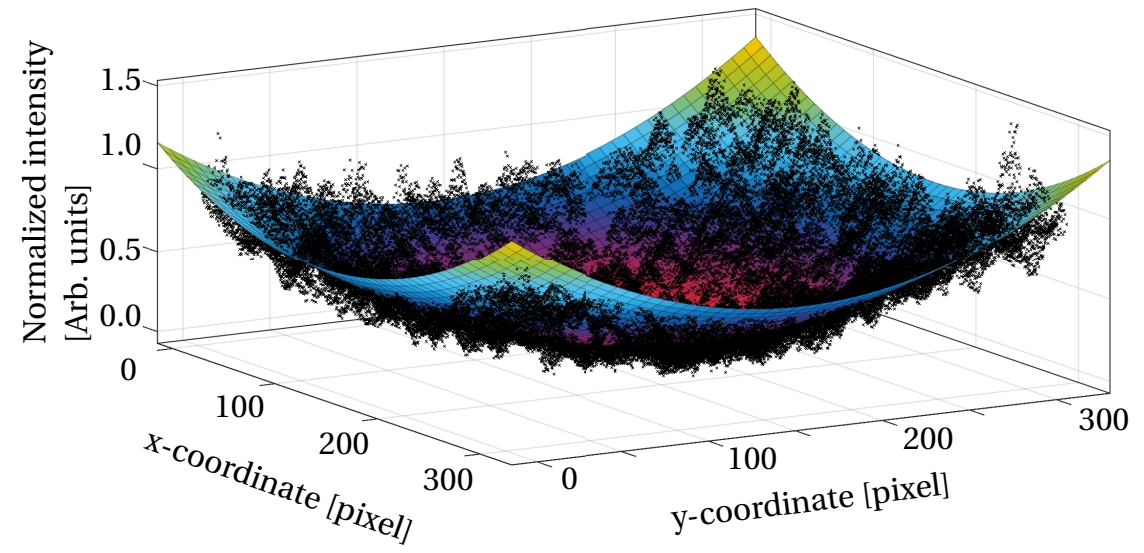

Figure 2.11: An example of a three dimensional quadratic surface fitted to an axisymmetric intensity profile to specify the center. 


\section{Chapter 3}

\section{Dynamic Leidenfrost effect: relevant time and length scales ${ }^{\dagger}$}

When a liquid droplet impacts a hot solid surface, enough vapour may be generated under it as to prevent its contact with the solid. The minimum solid temperature for this so-called Leidenfrost effect to occur is termed the Leidenfrost temperature, or the dynamic Leidenfrost temperature when the droplet velocity is non-negligible. We observe the wetting/drying and the levitation dynamics of the droplet impacting on an (isothermal) smooth sapphire surface using high speed total internal reflection imaging, which enables us to observe the droplet base up to about $100 \mathrm{~nm}$ above the substrate surface. By this method we are able to reveal the processes responsible for the transitional regime between the fully wetting and the fully levitated droplet as the solid temperature increases, thus shedding light on the characteristic time-and length-scales setting the dynamic Leidenfrost temperature for droplet impact on an isothermal substrate.

\footnotetext{
${ }^{\dagger}$ Published as: Minori Shirota*, Michiel A.J. van Limbeek* , Chao Sun, Andrea Prosperetti, and Detlef Lohse, Physical Review Letters 116, 064501 (2016)

* Both authors contributed equally on this publication
} 


\subsection{Introduction}

Boiling and spreading of droplets impacting on hot substrates have been extensively studied since both phenomena strongly affect the heat transfer between the liquid and the solid. Applications include spray cooling [13], spray combustion [63], and others [64].

At room temperature, an impacting droplet spreads on a solid surface, and entraps a bubble under it $[30,53,65]$. At temperatures higher than the boiling temperature $T_{\mathrm{b}}$, vapour bubbles appear, which disturb and finally rupture the free surface, resulting in the violent spattering of tiny droplets $[8,66]$, see also chapter 6 . On even hotter surfaces, however, beyond the so-called Leidenfrost temperature $T_{\mathrm{L}}$, the droplet interface becomes smooth again without any bubbles inside it. In this regime the droplet lives much longer as now it levitates on its own vapour layer: the well-known Leidenfrost effect $[15,36]$.

In order to determine the Leidenfrost temperature $T_{\mathrm{L}}$ and its dependence on the impact velocity $U$, phase diagrams have been experimentally produced for various impacting droplets with many combinations of substrates and liquids: water on smooth silicon [8], water on micro-structured silicon [50], FC-72 on carbon-nanofiber [67], water on aluminium [68], and ethanol on sapphire [69]. All these phase diagrams show a weakly increasing behaviour of $T_{\mathrm{L}}$ with $U$. When theoretically deriving $T_{\mathrm{L}}$, one needs to determine the vapour thickness profile. In the case of a gently deposited droplet, this can be accomplished since the shape of the droplet is fixed except for the bottom surface, which reduces the problem to a lubrication flow of vapour in the gap between the substrate and the free-surface $[14,16,17,21,27,70]$.

For impacting droplets on an unheated surface at high Weber number $W e \equiv$ $\rho U^{2} D_{0} / \sigma$ (here $D_{0}$ is the equivalent diameter of droplet and $\rho$ and $\sigma$ are the density and the surface tension of the liquid, respectively) it is known that the neck around the dimple beneath the impacting droplet rams the surface. In this cold impact case, the neck propagates outwards like a wave [71]. For impact on a superheated surface, however, it is not yet clear whether the neck still forms and rams the surface since the evaporation of the liquid in the neck and the resultant high pressure below the neck might smoothen out the structure, resulting in a circular vapour disk with a roughly homogeneous thickness.

The goal of the present paper is to experimentally clarify how the structure of the droplet base changes with increasing substrate temperature, i.e., how the characteristic time- and length-scales change at the transition from contact to Leidenfrost boiling. In order to explore how these scales change when undergoing the transition from contact to the Leidenfrost regime, we employed total internal reflection (TIR) imaging (see Fig. 3.1), which is a powerful technique to quantitatively evaluate the approach of impacting droplets on an evanescent length scale, typically $100 \mathrm{~nm}$ [53-55], and to clearly distinguish the wetted area from vapour bubbles/patches on heated substrates $[13,56]$.

Next to the impact velocity $U$, a key process that significantly affects $T_{\mathrm{L}}$ is the cooling of the substrate due to its exposure to the cold liquid. $T_{\mathrm{L}}$ thus strongly depends on the thermo-physical properties of both the liquid and the substrate used $[10,67,72]$. For example, a gently deposited ethanol droplet can achieve the Leidenfrost state at $T_{\mathrm{L}, \text { static }}=157^{\circ} \mathrm{C}$ on polished aluminium, whereas on pyrex glass a temperature as high 


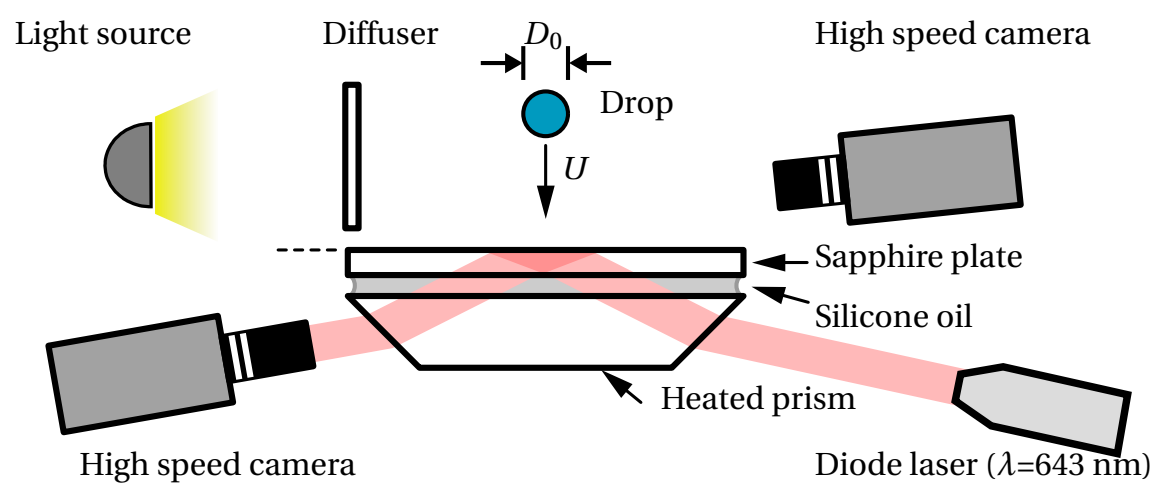

Figure 3.1: Schematic of the experimental setup with synchronized side-view and TIRimaging.

as $360{ }^{\circ} \mathrm{C}$ is required. Water droplets, with a latent heat double that of ethanol, touch down on glass even at $700{ }^{\circ} \mathrm{C}[10]$. A unique wetting pattern was found on a glass substrate heated at temperatures just below $T_{\mathrm{L}}[56]$.

\subsection{Experimental setup}

In this study, to avoid the complexity due to the cooling effects, we chose a combination of substrates and liquids to approximate isothermal conditions during droplet impact. We used sapphire, which has almost the same thermal properties as stainless steel, as heated surface and as liquids we used either ethanol or fluorinated heptane, the latter one of which has very low latent heat. With these materials, FTIR-imaging allowed us to reveal the boiling characteristics at the base of impacting droplets on isothermal substrates ranging from contact boiling to Leidenfrost boiling.

Droplets were released from a needle, which was connected to a syringe pump. We released droplets of two different liquids: ethanol and fluorinated heptane $\left(F_{16} C_{7}\right)$, commonly known as FC-84. The liquids have almost the same boiling temperature $T_{\mathrm{b}}$ $\left(\approx 80^{\circ} \mathrm{C}\right.$ ) but different latent heats $L: 853 \mathrm{~kJ} / \mathrm{kg}$ and $81 \mathrm{~kJ} / \mathrm{kg}$ for ethanol and FC-84, respectively. The generated droplets had a typical diameter $D_{0}$ of $2.8 \mathrm{~mm}$ for ethanol and $1.8 \mathrm{~mm}$ for FC-84. The impact velocity $U$ was varied by adjusting the needle height above the substrate in the range between $0.01 \mathrm{~m}$ and $1.5 \mathrm{~m}$ (measured from the droplet base), spanning the range of $U=0.4 \mathrm{~m} / \mathrm{s}$ and $4.3 \mathrm{~m} / \mathrm{s}$. Both $D_{0}$ and $U$ were measured with a high speed camera (Photron Fastcam SA1.1) at $10000 \mathrm{fps}$ with a macro lens. For bottom view observations, we employed FTIR-imaging by using a high-speed camera (Photron Fastcam SA-X2) at 40000 fps with a long-distance microscope (Navitar 12x Telecentric zoom system). Both side and bottom view images provided fields of view of about $10 \times 10 \mathrm{~mm}^{2}$, and spatial resolution of about $20 \mu \mathrm{m} /$ pixel.

The droplets impacted on a smooth sapphire substrate $(50 \mathrm{~mm}$ in diameter and 
(a) $T_{\mathrm{s}}=150^{\circ} \mathrm{C}$ (Contact boiling)
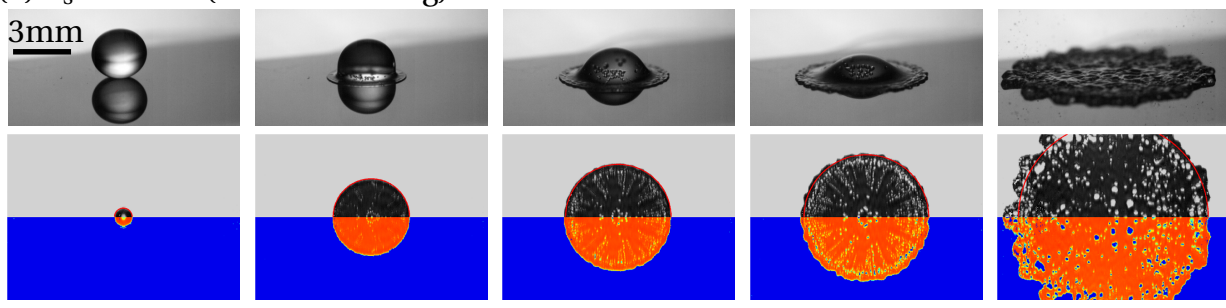

(b) $T_{\mathrm{s}}=170^{\circ} \mathrm{C}$ (Transition boiling)
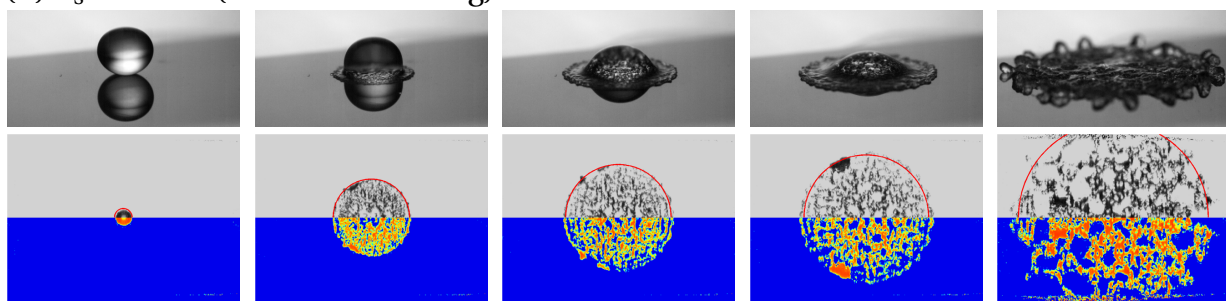

(c) $T_{\mathrm{s}}=180^{\circ} \mathrm{C}$ (Transition boiling)
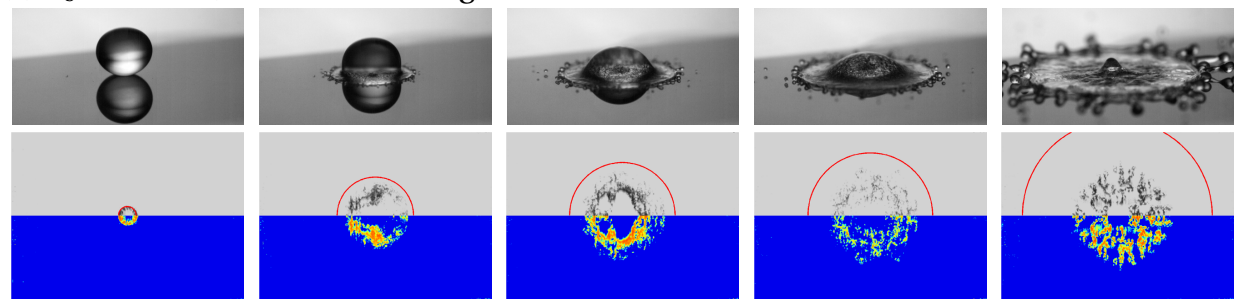

(d) $T_{\mathrm{s}}=220^{\circ} \mathrm{C}$ (Leidenfrost boiling)

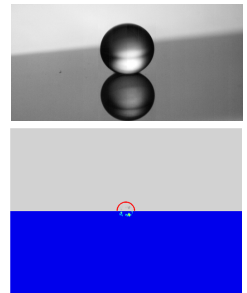

$t=0 \mathrm{~ms}$
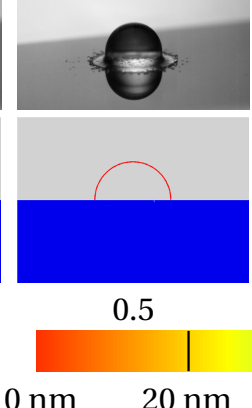
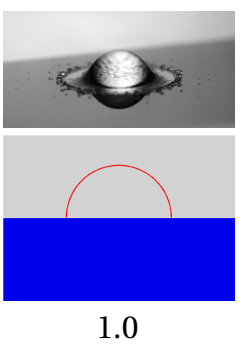

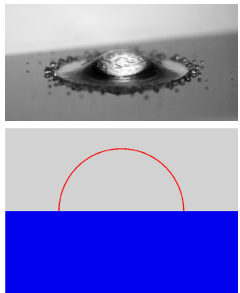

1.5

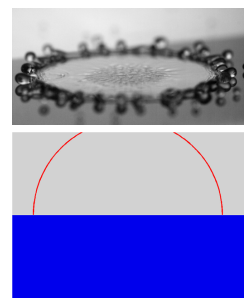

6.5 
Figure 3.2: Sequence of ethanol droplets impacting on a sapphire substrate at $T_{\mathrm{s}}=$ (a) 150, (b) 170 , (c) 180 and (d) $220^{\circ} \mathrm{C}(U=1.3 \mathrm{~m} / \mathrm{s}$ for all cases). The columns show images at different elapsed times after the impact; $t=0,0.5,1.0,1.5$ and $6.5 \mathrm{~ms}$ from left to right. The images in the upper-row in each pair show the side view, while the lower one consists of FTIR-images in original gray scale (upper part) and calculated color height scale (lower part; the image is the same as the upper one but horizontally flipped). The colored FTIR-images show the distance from the substrate surface according to the height map shown in the right bottom. The cut-off height is $91 \mathrm{~nm}$, and any distances more than this is shown in blue corresponding to the largest thickness. The wet area measured from the FTIR-images of $T_{\mathrm{s}}=150^{\circ} \mathrm{C}$ are drawn by red half circles as a measure of the diameter of the spreading front. The corresponding movies are available as supplementary material [73].

$3 \mathrm{~mm}$ in thickness) with a roughness of $10 \mathrm{~nm}$ (measured by AFM). The substrate was placed on a glass dove prism with a high-viscous silicone oil (kinematic viscosity: $0.01 \mathrm{~m}^{2} / \mathrm{s}$ ) between them for optical impedance matching (Fig. 3.1). The prism was mounted in an aluminium heating block whose temperature was PID-controlled to a fixed value ranging from $80^{\circ} \mathrm{C}$ to $590^{\circ} \mathrm{C}$ by two electrical heating cartridges and a thermal probe. The exact temperature on the substrate surface was measured before the experiment with a surface probe. For FTIR-imaging, a diode laser beam (wave length: $643 \mathrm{~nm}$ ) was expanded to about $20 \mathrm{~mm}$ in diameter, and introduced to the dove prism via mirrors at a certain incident angle. Since the intensity of an evanescent light exponentially decays with a distance from the substrate [52], the logarithmic intensity of the droplet image normalized by the one without the droplet is proportional to the distance. The proportionality is determined by the wave length and the incident angle of the laser, and the reflective indices of the substrate and the gas above the substrate. The analysis was corrected for multiple reflections as described in chapter 2 . When the droplet touches the substrate, the corresponding part of laser light transmits through the droplet, and therefore we can clearly distinguish the wetted area from the dry one as a sharp change in gray-scale intensity. The resulting image of the contacting droplet is not a circle but an ellipsoid since the image shrinks only in the direction along the side wall of the prism with the oblateness according to the incident angle. From the oblateness of the droplet image, therefore, we can calculate the incident angle which is the key parameter to quantitatively evaluate the decaying length of an evanescent wave (see chapter 2). The relative uncertainty is found to be $10 \%$ for the ethanol and $7 \%$ for the FC-84 measurements.

\subsection{Results and Discussion}

We observed in detail the behaviour of different boiling regimes with both side view and FTIR images for ethanol. Figure 3.2 shows the side view and FTIR-images. The latter consist of original gray scale images (upper part) and color height images (lower part). Just above the boiling temperature $T_{\mathrm{b}}$, from the color height images, we can clearly see that the droplet completely wets the substrate except for the area of the 
(a) Boiling regimes

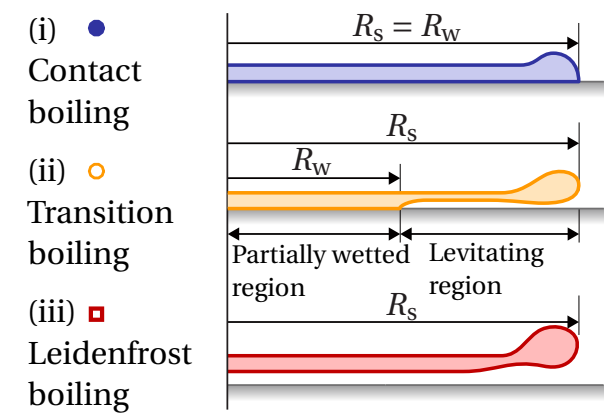

(b) Ethanol

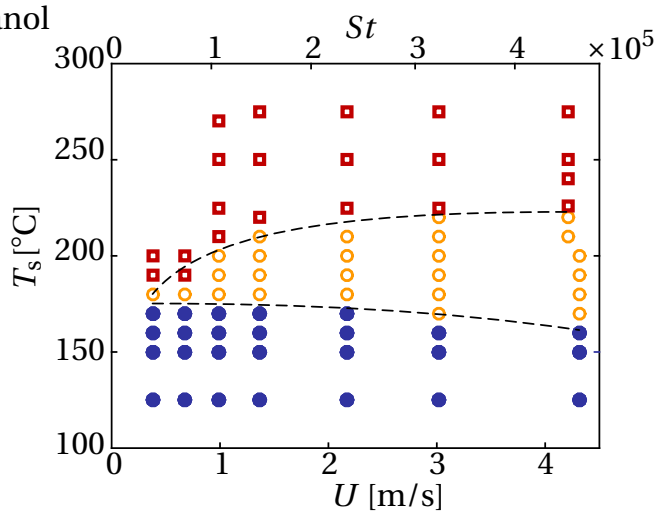

(c) FC-84

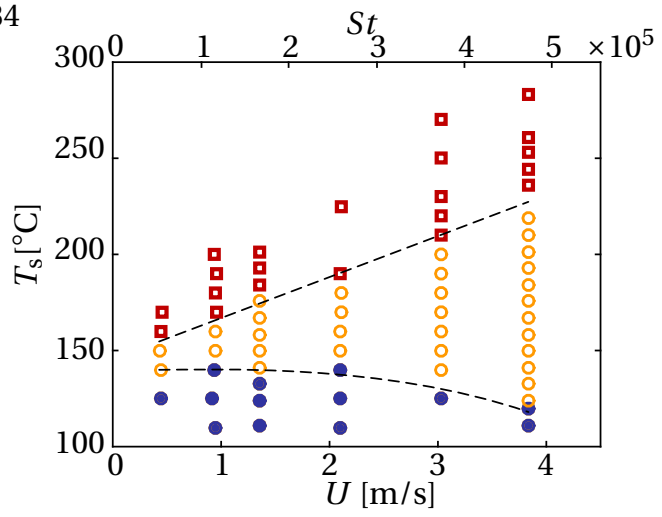

Figure 3.3: (a) Schematic diagrams of the three different boiling regimes identified in the present study, (i) contact, (ii) transition, and (iii) Leidenfrost, showing the differences between the radius of the spreading front $R_{\mathrm{S}}$ and that one of the (partially) wetting region $R_{\mathrm{w}}$. Phase diagram of the boiling regimes for (b) ethanol and (c) FC-84 with its more than ten times lower latent heat (as compared to ethanol). The plots indicate the three different boiling behaviors: the contact boiling regime (solid circle), the transition regime (open circle), and the Leidenfrost regime (square). The dashed lines between different regimes are drawn to guide the eye. For comparison, static Leidenfrost temperatures are $160{ }^{\circ} \mathrm{C}$ and $125^{\circ} \mathrm{C}$ for ethanol and FC-84, respectively. 
nucleated bubbles, see Fig. 3.2a. With increasing substrate temperature $T_{\mathrm{s}}$, the growth and coalescence of the nucleated bubbles are enhanced (Fig. 3.2b). Although at $t=0$ the droplet is in contact with the substrate aside from the central dimple region, only partial contact occurs later as indicated by smaller red areas and larger green areas in comparison with the lower temperature case. The color height images indicate the corrugated surface of the droplet base. However, the droplet surface is still within the length scale of evanescent light, $90 \mathrm{~nm}$ in this case. In addition, the radius of the periphery of the wetted area (red circle) is almost the same as that for $T_{\mathrm{S}}=150{ }^{\circ} \mathrm{C}$ (red circle). We thus categorize this regime still as contact boiling.

A further increase of the substrate temperature drastically changes the boiling behaviour, as sketched by the difference between Fig. 3.3 (a-i) and (a-ii). This regime, to which we refer as transition boiling, sets in at $T_{\mathrm{s}}=180^{\circ} \mathrm{C}$ (Fig. 3.2c). The FTIR images reveal that the wetted area is smaller than the spreading radius of the droplet, as shown by the wetted area being smaller than the red circle. The local evaporation in the outer area of the base causes the levitation of the lamella which becomes unstable and breaks up as shown in the side view images. The onset of the rim instability in the early stage of the impact (at $t=0.5 \mathrm{~ms}$ in Fig. 3.2c and d) is thus a good indicator for the end of the contact boiling regime. A close observation of the change in color height maps reveals that although most of the contact area is not wetted at $t=1.5 \mathrm{~ms}$, some contact (red area) is recovered later at $t=6.5 \mathrm{~ms}$. This can be explained by the cooling by the vapour from the droplet base located at about $50 \mathrm{~nm}$ from the substrate. We also found that there exists no fingering pattern in contrast to what was observed on glass substrates [56]. These results indicate that the fingering pattern is related to the cooling of the substrate by radially flowing vapour and subsequent re-wetting of the substrate.

At higher temperatures, the droplet never touches the substrate during the impact process, either temporarily or spatially: the Leidenfrost boiling regime sets in (Fig. 3.2d). In the side view images, we can also see that the levitation on the vapour layer changes the brightness of the droplet (cf. the four side view images at $t=1.0 \mathrm{~ms}$ in Fig. 3.2): here the backlight used for the side view imaging undergoes a total internal reflection at the top surface of the vapour layer, resulting in the bright color of the droplet in the Leidenfrost state.

In summary, on our nearly isothermal substrates, we have identified three different boiling regimes, i.e., contact-, transition-, and Leidenfrost boiling (Fig. 3.3a). In the contact boiling regime, the radius $R_{\mathrm{S}}$ of the spreading front coincides with that of (partially) wetted region $R_{\mathrm{W}}$, whereas in the transition boiling regime, $R_{\mathrm{W}}$ is smaller than $R_{\mathrm{s}}$. In the Leidenfrost boiling regime, $R_{\mathrm{W}}=0$ during the whole spreading process, i.e. there is no wetting at all. Note that the present classification of the boiling regimes is based on the direct observation of wet respective dry areas, and is thus different from the classification in previous studies $[8,50]$ where the boiling regimes were more superficially classified based on the smoothness of the droplet surface or the ejection of tiny droplets from the impacting droplet. On the basis of the definitions described above, the boiling regimes can be classified into contact, transition and Leidenfrost for impact velocities ranging from about 0.5 to $4 \mathrm{~m} / \mathrm{s}$ for both liquids. The phase diagrams of Fig. 3.3b and c show a considerable temperature range for transition boiling, approximately $50 \mathrm{~K}$ and $100 \mathrm{~K}$ for ethanol and FC-84, respectively.

For ethanol, the lower boundary between contact and transition boiling depends 
very weakly on $U$, if at all. The higher boundary between transition and film boiling, i.e. $T_{\mathrm{L}}$, increases with $U$ only weakly. In addition, our experiments with a sapphire substrate show a $T_{\mathrm{L}}$ lower by about $50 \mathrm{~K}$ as compared with glass [56]. This finding confirms that significant heat is lost from the substrate by cooling even during the short impact time. For FC-84, the contact-to-transition boundary is lower than that for ethanol due to its more than ten times lower latent heat as compared to ethanol. At low impact velocities, the upper $T_{\mathrm{L}}$ boundary is also lower than for ethanol, but it increases with $U$ and reaches almost the same level as that for ethanol at $U \approx 4 \mathrm{~m} / \mathrm{s}$. Several models for the spreading diameter $D_{\max }$ of an impacting drop have been developed [36, 38, 50,74]. For the so-called pancake model $D_{\max }$ and $D_{0} / U$ are considered to be the relevant length- and time-scales [50]. In addition, the model assumes a roughly homogeneous thickness for the vapour layer below the droplet. However, this model, combined with a force balance on the levitating droplet base (shear stress of vapour $\sim$ capillary pressure), does not correctly describe the dependence of $T_{L}$ on $U$, because it predicts a scaling $T_{\mathrm{L}}-T_{\mathrm{b}} \sim U^{-2}$, which is neither consistent with the current experimental results (Fig. $3.3 \mathrm{~b}$ and c) nor with previous ones $[8,50,67,68]$.

Our FTIR-images shown in Fig. 3.4a reveal the crucial deficiencies of the pancake model: just below $T_{\mathrm{L}}$, in the very beginning of the impact process $\left(t U / D_{0}<0.1\right)$ a circular ring of radius $R_{\mathrm{n}}(t)$ that is smaller than the undisturbed droplet radius $\left(R_{\mathrm{n}} / R_{0}<\right.$ 1) becomes visible within the evanescent length scale. Based on this finding as well as on the fact that the ring corresponds to the area of the droplet base closest to the substrate, we conclude that the ring shows the position of the neck around the central dimple, see the sketch shown as an inset of Fig. 3.4b. The pancake model is therefore inappropriate for the modelling of $T_{\mathrm{L}}$ because of its much larger time- and length-scales and the erroneous assumption of a roughly homogeneous vapour film thickness.

In their study of a droplet impacting on a cold surface, Riboux and Gordillo [44] have pointed out the similarity with the classical problem of a solid body impacting a liquid surface first treated by Wagner [32] (see also [33,34]). They show the relevance of Wagner's prediction $\sqrt{3 R_{0} U t}$ for the radius of the liquid-solid contact region for the droplet impact problem. The situation in the present case is different because the formation of the dimple and the associated neck precedes the actual liquid-solid contact. However, a strong similarity can be found in the very high pressure that develops under the droplet due to the vapour formation. This consideration has prompted us to examine the possible connection between the neck radius $R_{\mathrm{n}}$ on the lower surface of the droplet and Wagner's result.

The symbols in Fig. 3.4b are the measured position of the neck around the central dimple for four cases, while the lines show Wagner's result $\sqrt{3 R_{0} U t}$. The striking agreement for the two liquids, and various $T_{\mathrm{s}}, R_{0}$, and $U$ (Fig. 3.4b) suggests that the concept introduced by Riboux and Gordillo [44] for impact on a cold substrate is equally applicable to the present hot substrate case. This finding reveals that the relevant time- and length-scales for the droplet touch-down on a superheated solid surface are the dimple formation time $t_{\mathrm{d}} \sim h_{\mathrm{d}} / U$ and the radius of the neck $R_{\mathrm{n}}$. These scale as $t_{\mathrm{d}} U / R_{0} \sim h_{\mathrm{d}} / R_{0} \sim S t^{-2 / 3}$ and $R_{\mathrm{n}} / R_{0} \sim \sqrt{3 U t_{\mathrm{d}} / R_{0}} \sim S t^{-1 / 3}$, where we used the wellknown scaling for the dimple height (see the inset of Fig. 4 (b)) $h_{\mathrm{d}} \sim R_{0} S t^{-2 / 3}$ in the inertia-dominant regime $[30,71]$ with the Stokes number $S t=\rho R_{0} U / \mu_{\mathrm{v}}$ based on the vapour viscosity $\mu_{\mathrm{v}}$. 


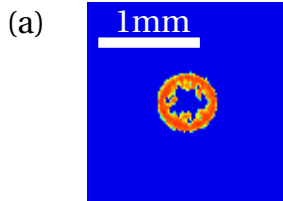

$0 \mathrm{~ms}$

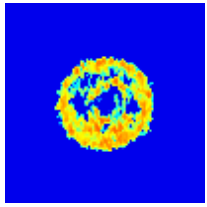

$0.05 \mathrm{~ms}$

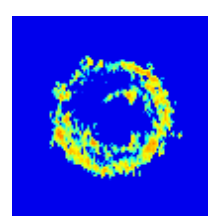

$0.1 \mathrm{~ms}$

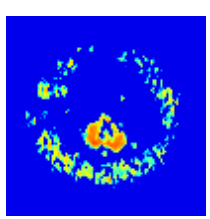

$0.15 \mathrm{~ms}$

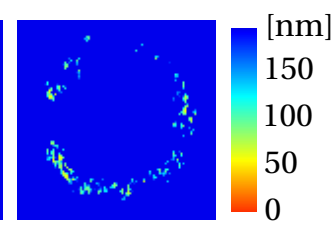

$0.2 \mathrm{~ms}$

(b)

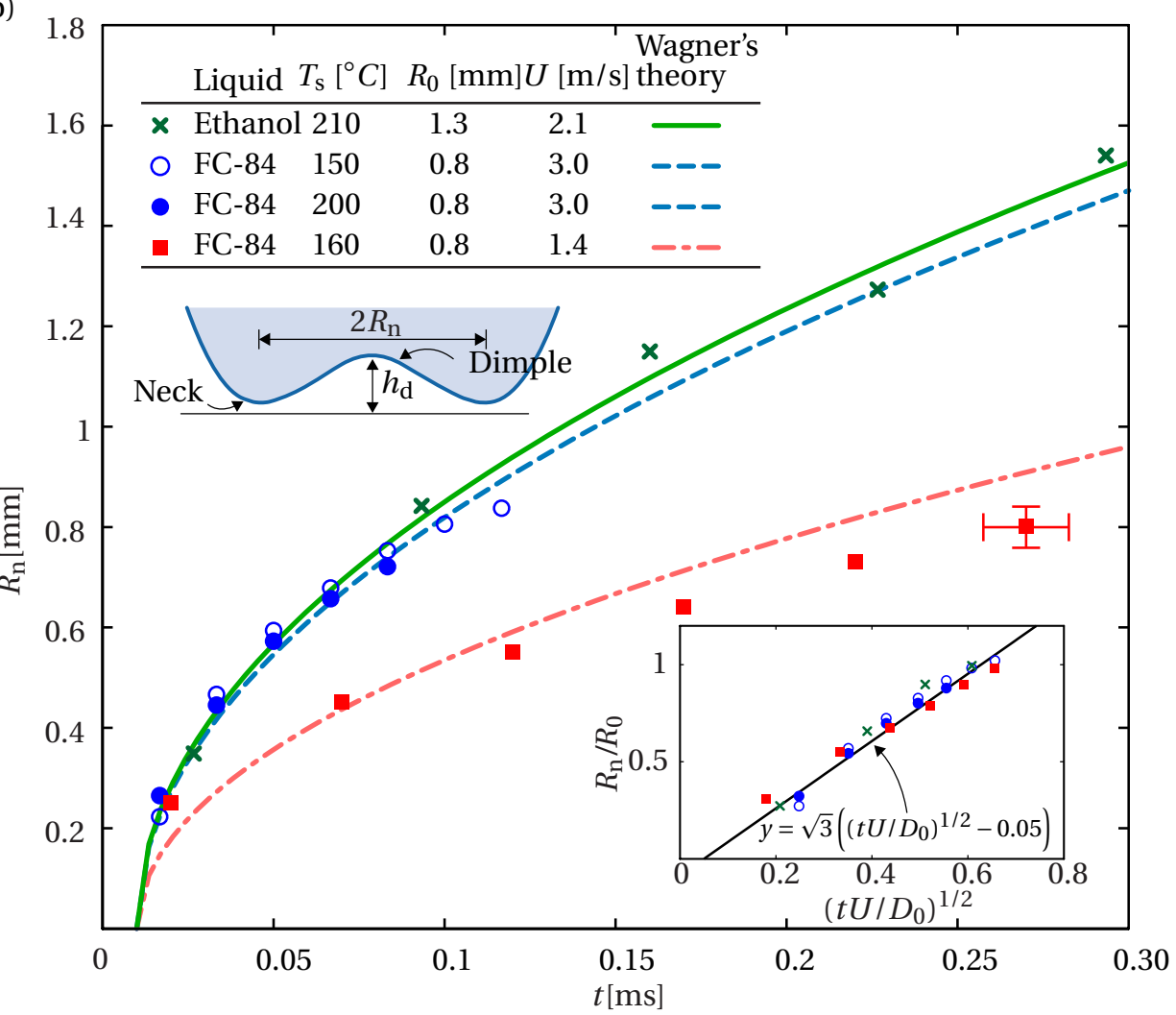

Figure 3.4: Spreading of a circular liquid ring within the evanescent length scale at the beginning of the impact. (a) Colour height images obtained from TIR-images of the droplet base, for FC-84, $T_{\mathrm{s}}=160{ }^{\circ} \mathrm{C}$ and $U=1.4 \mathrm{~m} / \mathrm{s}$. (b) Radius of the circular ring, $R_{\mathrm{n}}$, corresponding to the closest area of the droplet to the substrate. The error bars represent the uncertainty due to the frame interval in the imaging (horizontal) and the pixel resolution of the image (vertical). The curves show the position of the maximum pressure area, estimated by a modified Wagner's theory: $\sqrt{3 R_{0} U t}$. The inset shows the same data but in dimensionless form. The data collapse reveals the universality. 


\subsection{Conclusion}

In conclusion, the use of total internal reflection (FTIR) imaging has permitted us to resolve the processes occurring under an impacting liquid droplet within $100 \mathrm{~nm}$ above an isothermal superheated surface. We for the first time quantitatively evaluated the height of the droplet base, levitating on its vapour within the evanescence length scale in the transition regime between the contact and the Leidenfrost regimes. The data are presented in the form of phase diagrams in the temperature-velocity parameter space. At temperatures just below the Leidenfrost threshold $T_{\mathrm{L}}$, a circular liquid ring is seen to spread within the evanescent length scale at the beginning of the impact process. This observation provided us with the crucial ingredient for future modelling of $T_{\mathrm{L}}$, namely the importance of the dimple and neck structures under the droplet.

\section{Acknowledgements}

We thank Sander Wildeman for illuminating discussion. This work was partially supported by Fundamenteel Onderzoek der Materie and by an ERC-Advanced Grant. 


\section{Chapter 4}

\section{Boiling regimes of impacting drops on heated substrate at reduced pressure ${ }^{\dagger}$}

We experimentally investigated the boiling behaviour of impacting ethanol drops on a heated smooth sapphire substrate at pressures ranging from $P=0.13$ bar to atmospheric pressure. We employ Frustrated Total Internal Reflection (FTIR) imaging to study the wetting dynamics of the contact between the drop and the substrate. The spreading drop can be in full contact (contact boiling), it can partially touch (transition boiling) or the drop can be fully levitated (Leidenfrost boiling). We show that the temperature of the boundary between contact and transition boiling shows at most a weak dependency on impact velocity, but a significant decrease with decreasing ambient gas pressure. A striking correspondence is found between the temperature of this boundary and the static Leidenfrost temperature for all pressures. We therefore conclude that both phenomena share the same mechanism, and are dominated by the dynamics taken place at the contact line.

On the other hand, the boundary between transition boiling and Leidenfrost boiling, i.e. the dynamic Leidenfrost temperature, increases for increasing impact velocity for all ambient gas pressures. Moreover, the dynamic Leidenfrost temperature coincides for pressures between $P=0.13$ and $P=0.54$ bar, whereas for atmospheric pressure the dynamic Leidenfrost temperature is slightly elevated. This indicates that the dynamic Leidenfrost temperature is at most weakly dependent on the enhanced evaporation by the lower saturation temperature of the liquid.

\footnotetext{
${ }^{\dagger}$ Paul B.J. Hoefnagels*, Michiel A. J. van Limbeek*, Olinka J. Ramirez Soto, Chao Sun and Detlef Lohse, submitted for publication

* Both authors contributed equally on this publication
} 


\subsection{Introduction}

Drop impact on heated surfaces has been an interesting research topic due to its numerous applications, such as in spray cooling and fuel injection $[13,63]$. In these processes it is desirable to have a high heat transfer rate between the solid and the liquid which is achieved by a large contact area between the liquid and the substrate. When the substrate is heated above a critical temperature, the drop is hovering above the surface on its own vapour. The low thermal conductivity of the vapour layer insulates the liquid from the substrate and hence the heat transfer from the substrate to the drop is drastically reduced. As a result, the lifetime of a deposited drop increases significantly. The lowest substrate temperature $T_{\mathrm{s}}$ at which this phenomenon occurs is referred to as the Leidenfrost temperature $T_{\mathrm{L}}[4,7,15]$.

For droplets impacting a heated surface it is shown that $T_{\mathrm{L}}$ increases with increasing impact velocity $[8,36,75]$. Prior to impact a high pressure region is formed between the drop and the substrate, a result of the viscosity of the escaping air [76]. This causes a deceleration of the drop and the formation of a dimple in the drop before touchdown $[30,39]$. Touchdown of the drop on the substrate can be prevented completely in the case of a heated substrate, because the escaping air is replenished by the evaporation of the drop $[8,36,45]$.

Since it is known that the ambient gas pressure has a great influence on impact dynamics [41], it is interesting to study how heating the substrate affects touchdown in reduced pressure conditions. A major consequence of lowering the ambient gas pressure in the unheated case is the suppression of splash formation [41]. This phenomenon has been studied extensively both experimentally [31,41,53,77-79] and numerically [71,80], where a crucial role is found for both the wetting of the substrate and the air-liquid interaction [44].

The goal of this study is to find the influence of the ambient gas pressure on the wetting dynamics of impacting ethanol drops on a sapphire substrate.

\subsection{Experimental methods}

To study the impact behaviour of ethanol drops on a heated surface at various pressures, a FTIR (Frustrated Total Internal Reflection) setup was used as shown in Fig. 4.1. Details of the setup and methods described in chapter 2. For self-consitency, here we give the essential aspects. A laser beam (wavelength $\lambda=643 \mathrm{~nm}$, p-polarized) was widened by a beam expander and illuminated the sapphire-air surface. The angle of the incident light was larger than the critical angle to reflect the laser beam. The light was recorded with a high speed camera (Photron SA-X2) with a frame rate of at least $20000 \mathrm{fps}$, and the camera was focused on the top interface of the prism. When a drop was in contact with the surface, a fraction of the laser beam can be transmitted across the interface, resulting in the detection of a dark spot by the camera. Intermediate intensities of the reflected light appeared when the base of the drop was just above the surface within the distance of the decay length of the evanescent wave, typically in the order of hundreds of $\mathrm{nm}$. A sapphire substrate was chosen because of its transparency, its smoothness (surface roughness of $10 \mathrm{~nm})$ and its relatively high thermal conductivity of $k_{\mathrm{s}}=32 \mathrm{~W} /(\mathrm{mK})$. The sapphire prism was positioned in an aluminium holder that was kept at constant 
temperature using a proportional-integral-derivative controller. The temperature of the sapphire surface $T_{\mathrm{s}}$ was calibrated by using a surface probe as well as a Pt-100 sensor for various ambient pressures and heater set points.

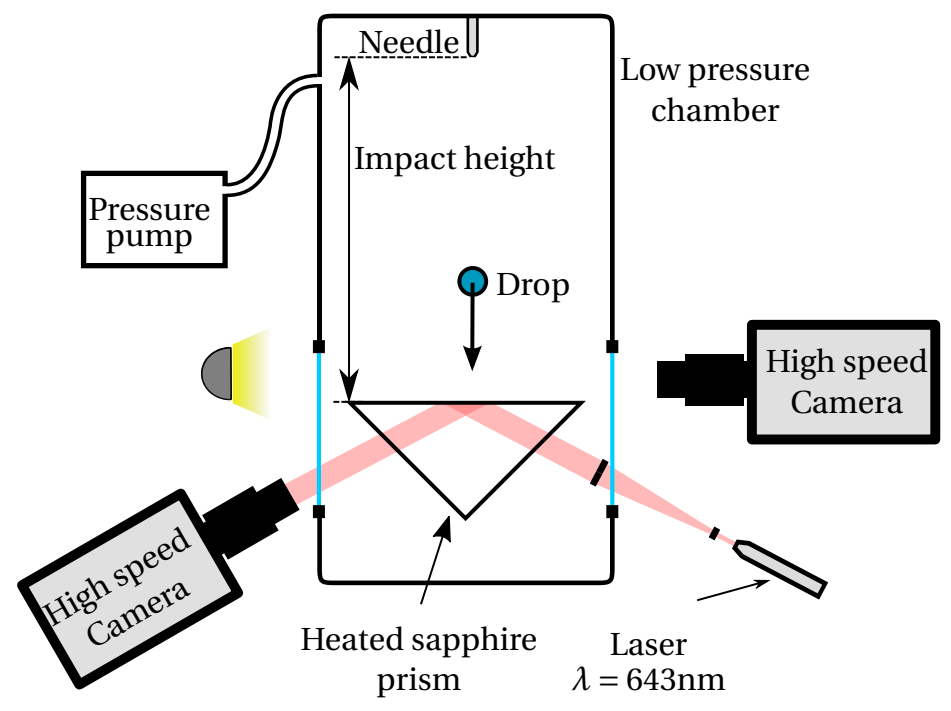

Figure 4.1: Schematic representation of set-up used in our experiments. In addition to a side-view camera, we study the wetting of the drop by a bottom-view camera, using frustrated total internal reflection imaging. The sapphire prism was heated to set the desired surface temperature $T_{\mathrm{s}}$.

The needle and heated surface were situated in a low pressure chamber. The surrounding pressure ranging from $P=0.13 \mathrm{bar}$ to atmospheric pressure, was controlled by a pump and a pressure sensor (JUMO dTrans p30). The chamber had a volume of about $30 \mathrm{~L}$, so the evaporated ethanol drops had a negligible effect on the gas composition. The needle was surrounded by a brass block that was maintained at a constant temperature of $5^{\circ} \mathrm{C}$ to ensure that the ethanol was well below the saturation temperature at all pressures. Single drops of diameter $D_{0}=2.6 \mathrm{~mm}$ were created from the tip of the needle and fell down when the gravitational force on the drop overcame the surface tension. The impact velocity $U$ on the substrate was varied between $U=0.3$ and $1.7 \mathrm{~m} / \mathrm{s}$ by varying the height of the needle. $D_{0}$ and $U$ were measured by tracking the drop $4 \mathrm{~mm}$ before impact using a secondary high speed camera (Photron SA1.1) at $20000 \mathrm{fps}$. The light sources and cameras were placed outside of the low pressure chamber and the images were recorded through a glass window in the chamber.

\subsection{Results and discussion}

\subsubsection{Phase diagram}

We studied in detail the boiling behaviour of the impacting ethanol drops on the heated surface using the FTIR images. These can be categorized into three boiling regimes: 
contact boiling (CB), transition boiling (TB) and Leidenfrost boiling (LF). At sufficiently low substrate temperatures $T_{\mathrm{s}}$, the wetting front of the drop has the same velocity as that of a drop impacting an unheated surface [45]. This is identified as the contact boiling state, see Fig. 4.2a. At certain $T_{\mathrm{s}}$ this is no longer observed: the spreading radius $R_{\mathrm{S}}$ of the drop exceeds the wetting radius $R_{\mathrm{W}}$ of the liquid on the surface. The edge of the drop is levitated above the surface, called the lamella, and this behaviour is categorized as transition boiling [45], see Fig. 4.2b. At even higher values of $T_{\mathrm{s}}$ the impacting drop does not wet the surface at all, hence it is in the Leidenfrost state, as shown in Fig. 4.2c. The temperature at which a change in boiling behaviour occurs between contact and transition boiling will be referred to as the CB-TB boundary. Similarly, the TB-LF boundary refers to the temperature at which drop becomes in the Leidenfrost state.

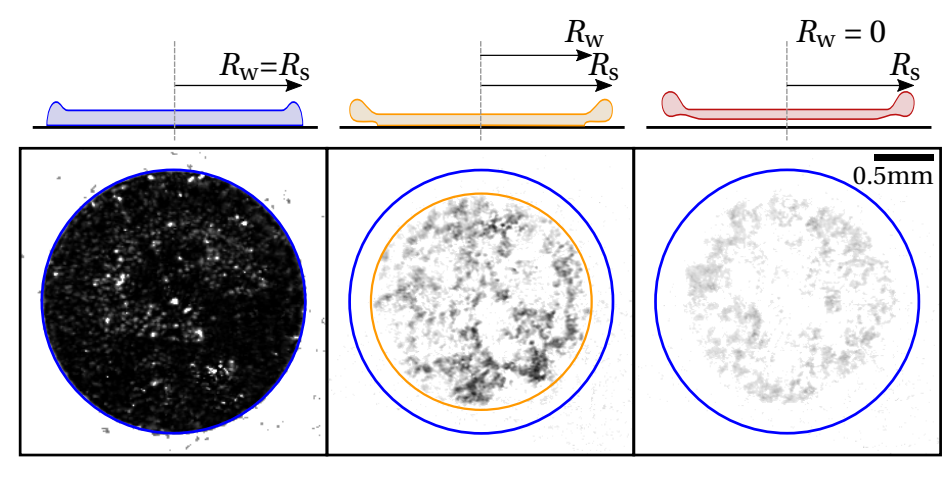
(a) $T_{\mathrm{s}}=138^{\circ} \mathrm{C}$
(b) $T_{\mathrm{s}}=181^{\circ} \mathrm{C}$
(c) $T_{\mathrm{s}}=199^{\circ} \mathrm{C}$

Figure 4.2: FTIR images of ethanol droplets at $t=0.21 \mathrm{~ms}$ after impacting a sapphire prism with impact velocity $U=1.0 \mathrm{~m} / \mathrm{s}$ at atmospheric pressure. For a substrate temperature of $T_{\mathrm{s}}=138^{\circ} \mathrm{C}$ the spreading drop fully wets the surface and is in contact boiling. At $181^{\circ} \mathrm{C}$ the droplet partially wets the surface and identified as transition boiling. At $199^{\circ} \mathrm{C}$ the drop is only visible in gray values: it is fully levitated and hence in the Leidenfrost boiling state. The blue circles correspond to the radius of the wetted area of a drop in the contact boiling state.

A phase diagram for ethanol drops with different impact velocities $U$ and surface temperatures $T_{\mathrm{s}}$ at atmospheric pressure is shown in Fig. 4.3. The temperature for the CB-TB boundary is at most weakly dependent of $U$, while the temperature of the TB-LF boundary, i.e. the dynamic Leidenfrost temperature, increases for higher $U$. This is in agreement with previous results for different liquids $[45,57]$.

It is interesting to study these boiling boundaries at different pressures, since it has been shown that the dynamics during drop impact can change drastically in low pressure conditions $[31,41,53,71,77-80]$. We therefore perform similar experiments at low pressure and the observed CB-TB and TB-LF boundaries are shown in Fig. 4.4. 


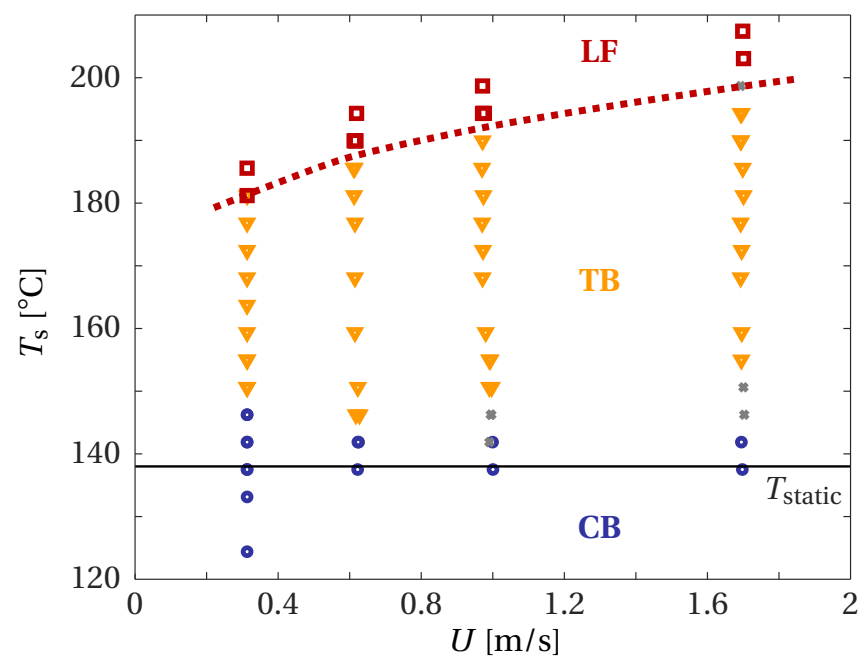

Figure 4.3: Phase diagram for ethanol drops impacting on sapphire substrate at atmospheric gas pressure. The blue diamonds correspond to contact boiling, yellow triangles to transition boiling and red squares to the Leidenfrost state. The grey crosses indicate measurements for which the boiling state is hard to identify. The red dotted line is a guide to the eye for the TB-LF boundary: the dynamic Leidenfrost temperature. The horizontal black line indicates the static Leidenfrost temperature on a silicon substrate for comparison.

\subsubsection{Boundary between the contact and transition boiling regime}

For all pressures the CB-TB boundaries are within the measurement error independent of impact speed, in correspondence with the trend found for measurements at atmospheric pressure. However, the temperature of the CB-TB boundary lowers with decreasing ambient gas pressure. In literature this boundary is correlated with the static Leidenfrost temperature $T_{\mathrm{L}}[45,57]$. Therefore we compare this boundary with $T_{\mathrm{L}}$ for different pressures on a silicon substrate. Because of its high thermal conductivity $k_{\mathrm{s}}=148 \mathrm{~W} /(\mathrm{mK})$ at $T=300 \mathrm{~K}$, the silicon substrate is considered to be isothermal [10] during the evaporation of the drop. The pressure dependence of $T_{\mathrm{L}}=T_{\mathrm{L}}(P)$ is shown in Fig. 4.5 and shows a similar trend as the saturation temperature of ethanol.

Since the saturation temperature is well described by Clausius-Clapeyron equation, we aim to describe $T_{\mathrm{L}}$ by a function of a similar form:

$$
\frac{1}{T_{\mathrm{L}}}=A-B \cdot \log _{10}(P)
$$

as suggested before by Orejon et al. [81]. For our results we find $A=0.0069 \pm 0.0005$ and $B=0.004 \pm 0.001$. Indeed, the agreement between $T_{\mathrm{L}}(P)$ and the CB-TB boundary is striking, suggesting that the latter is determined by the same physical mechanisms as $T_{\mathrm{L}}(P)$. 


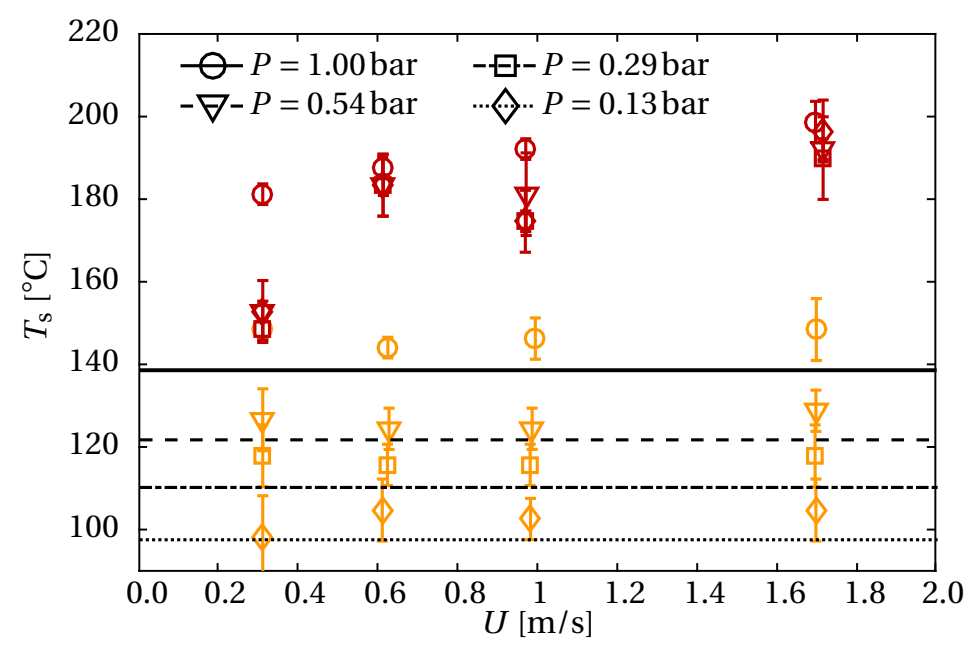

Figure 4.4: The CB-TB boundaries for different pressures are shown in yellow and the dynamic Leidenfrost temperatures are shown in red for various impact velocities while varying the ambient gas pressure. The horizontal black lines show the static Leidenfrost temperature on a silicon substrate at the different pressures, correlating well with the CB-TB boundary.

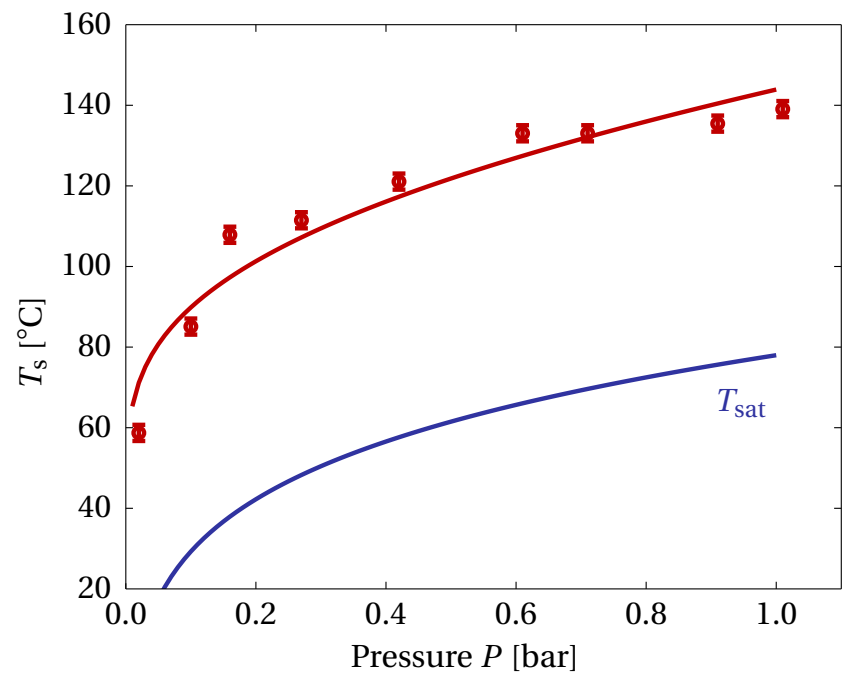

Figure 4.5: The static Leidenfrost temperature $T_{\mathrm{L}}$ of ethanol drops on silicon change with ambient pressure, as shown in red circles. As argued in the text, a logarithmic function (Equation 4.1) is fitted through these points. The dependence of the saturation temperature on the pressure of ethanol is shown as blue solid line. 


\subsubsection{Pressure effect on the dynamic Leidenfrost temperature}

An interesting behaviour of the dynamic Leidenfrost temperature is found when varying the ambient gas pressure: we find a collapse for impacts at ambient gas pressures between $P=0.13$ and $P=0.54$ bar, as shown in Fig. 4 .4. When comparing the dynamic Leidenfrost temperature in reduced pressures with our results at atmospheric conditions (subsection 4.3.1) we find a slightly lower temperature. On the other hand, the dynamic Leidenfrost temperature lowers with decreasing impact velocity for all ambient gas pressures.

It highlights that the impact dynamics are dominated by the high pressure developing in the drop [32-35], rather than the pressure in far field. The complexity of the dynamic Leidenfrost problem is now further increased due to the compressibility of the gas in reduced pressure conditions [71]. Although an enhancement of the evaporation is expected as a result of the lower saturation temperature in reduced pressure conditions, our observations indicate that this does not play a dominant role for the dynamic Leidenfrost temperature. We conclude that the ambient gas pressure has minor influence on the local pressure underneath the drop and thus on the vapour pressure and evaporation rate. Numerical work showed that at lower ambient gas pressures the shape of the impacting drop changes and the neck widens [71]. Since a larger area is close to the wall, an enhanced vapour generation might be expected, compensating the lower content of air at reduced pressure conditions, but the current work does not allow us to draw firm conclusions on this matter.

\subsection{Conclusion and Outlook}

In this study we identified the boiling regimes for ethanol drops on a sapphire substrate for different impact velocities and ambient gas pressures. High speed FTIR imaging is used to observe the wetting of the impacting drops.

The temperature of the boundary between contact boiling and transition boiling systematically decreases when reducing the ambient gas pressure. It showed excellent agreement with the Leidenfrost temperature found here for static drops, from which we conclude that they share the same underlying mechanism. This boundary remained approximately independent of impact velocity, in agreement with previous studies.

On the other hand, we found a collapse of the dynamic Leidenfrost temperature at reduced pressure conditions, slightly below the dynamic Leidenfrost obtained in atmospheric conditions.

Combining these two observations reveals that the temperature range where transition boiling is found strongly increases with decreasing pressure. It would be interesting to extend this study to elevated pressures to investigate how this trend develops above atmospheric conditions. One might find a collapse of both boundaries, resulting in the disappearance of the transition boiling regime.

\section{Acknowledgements}

This work was supported by an ERC-Advanced Grant. 


\section{Chapter 5}

\section{Vapour cooling of poorly conducting hot substrates increases the dynamic Leidenfrost temperature $^{\dagger}$}

A drop impacting a smooth solid surface heated above the saturation temperature can either touch it (contact boiling) or not (film boiling), depending on the surface temperature. The heat transfer is greatly reduced in the latter case by the insulating vapour layer under the drop. In contrast to previous studies, here we use a relatively poor thermally conducting glass surface. Using a total internal reflection method, we visualise the wetting dynamics of the drop on the surface. We discover a new touch-down process, in which liquid-solid contact occurs a few hundred microseconds after the initial impact. This phenomenon is due to the cooling of the solid surface by the generation of vapour. We propose a model to account for this cooling effect, and validate it experimentally with our observations. The model leads to the determination of a thermal time scale (about $0.3 \mathrm{~ms}$ for glass) for the cooling of the solid. We conclude that when the impact time scale of the drop on the substrate (drop diameter/impact velocity) is of the order of the thermal time scale or larger, the cooling effect cannot be neglected and the drop will make contact in this manner. If the impact time scale however is much smaller than the thermal time scale, the surface remains essentially isothermal and the impact dynamics is not affected.

\footnotetext{
${ }^{\dagger}$ Published as: Michiel A.J. van Limbeek* , Minori Shirota*, Pascal Sleutel, Chao Sun, Andrea Prosperetti and Detlef Lohse, International Journal of Heat and Mass Transfer 97, p101-109 (2016)

* Both authors contributed equally on this publication
} 


\subsection{Introduction}

In spray cooling applications and others, it is necessary to maintain contact between the droplets and the heated surface. As has been known for centuries [7,15], contact is abruptly lost once the surface of the hot solid exceeds a rather well defined value, called the Leidenfrost temperature $T_{\mathrm{L}}$. This temperature depends on the thermo-physical properties of the liquid and the solid $[10,18]$, the micro-structure of the surface $[82,83]$ and the impact velocity $U$ [8]. In the static Leidenfrost case $(U=0)$, in which no impact dynamics are present, the lifetime of the drop is much longer than a thermal time scale $\tau_{\text {th }}=k_{\mathrm{s}} \rho_{\mathrm{s}} C p_{\mathrm{s}} h^{-2}[4,10,17]$. Here, $\rho_{\mathrm{s}}$ is the solid density, $k_{\mathrm{s}}$ the thermal conductivity [W/ $(\mathrm{Km})], C p_{\mathrm{s}}$ the specific heat and $h$ the heat transfer coefficient from the solid to the drop. In general, the local cooling of the solid produced by the drop causes the Leidenfrost temperature $T_{\mathrm{L}}$ to depend on the solid's thermal properties $[10,17]$. However, if the drop lifetime is shorter than $\tau_{\text {th }}$, the solid remains essentially isothermal and $T_{\mathrm{L}}$ reaches a lower limiting value.

When the impacting drop has an initial downward velocity $U \neq 0$, the phenomenon is referred to as dynamic Leidenfrost effect. Due to its widespread applications, this dynamic case has drawn much interest [50,56,67,69,73,83-85]. By the dynamic pressure of the impact, the drop partly overcomes the cushioning of the vapour layer and only touches down at plate temperatures higher than the static $T_{\mathrm{L}}$. The dynamic Leidenfrost temperature is found to depend on the drop size, impact velocity, liquid properties, surface roughness [50,67] and solid vibrations [85]. Some studies investigate scaling laws for the maximum radius of the deforming drop $[50,67,86]$ or models for the time evolution of the impact dynamics [73, 74, 87].

The focus of this paper is the study of impacts on the smooth surface of glass, which has a relatively poor thermal conductivity and diffusivity, as little is known about how these features affect $T_{\mathrm{L}}$. We experimentally examine the liquid-solid touch-down mechanism and how it is affected by the limited heat transfer supplied by the glass. The impacting liquid drop consists of ethanol and has an initial size $D_{0}$ and velocity $U$. We measure the thermal time scale $\tau_{\text {th }}$ and compare it with the impact time scale $\tau_{\text {imp }}=D_{0} / U$. At times larger than $\tau_{\text {imp }}$ all downward momentum is transferred into the radial direction and the drop is no longer forced into contact with the substrate. Our data shows that when $\tau_{\text {th }}$ is larger than $\tau_{\text {imp }}$, the substrate remains essentially isothermal. In the opposite case, the surface is appreciably cooled down by the (partial) evaporation of the drop and the cooling caused by the drop cannot be neglected. By using microdroplets instead of millimetre sized drops, we recover an isothermal impact, as $\tau_{\text {imp }}=D_{0} / U$ is then decreased by the decrease of the drop size.

\subsection{Experimental setup and procedure}

As sketched in Fig. 5.1, the experimental setup consists of a drop generator, a smooth and dry glass surface on which the drops impact, and two cameras to study the phenomenon.

Drops with a diameter between 2.1 and $2.6 \mathrm{~mm}$ are released from a needle from a variable height. The resulting impact velocity ranges between $0.4 \mathrm{~m} / \mathrm{s}$ and $3.9 \mathrm{~m} / \mathrm{s}$, with corresponding Weber numbers $W e=\rho_{\mathrm{l}} U^{2} D_{0} / \sigma_{1}$ between 16 and 1480; here $\rho_{\mathrm{l}}$ 


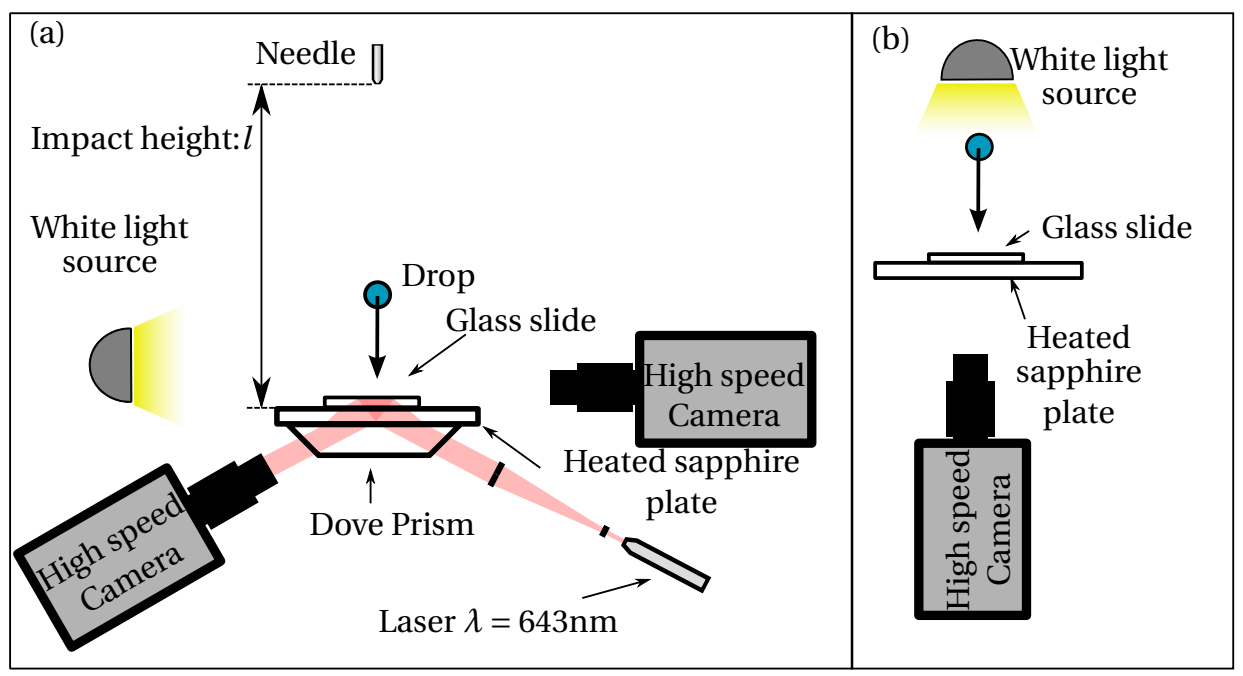

Figure 5.1: Schematic of the experimental setups used: the total internal refraction (TIR) setup (a) and the bottom view setup (b).

and $\sigma_{1}$ are the liquid density and surface tension coefficient. We also study the impact of $300 \mu \mathrm{m}$-diameter microdroplets, which are generated as a continuous stream by an inkjet nozzle, with an impact velocity of $8.9 \mathrm{~m} / \mathrm{s}$. The stream is blocked by mechanical shutters, which can open briefly to allow a few drops to reach the surface. This arrangement also scatters the drops in space, so that they do not interact with each other during impact. In all experiments we measured both the diameter and impact velocity of each drop with a high speed camera (Photron Fastcam SA1.1) at $10000 \mathrm{fps}$.

The ethanol drops impact a smooth glass microscope slide, the properties of which are listed in Tab. 5.1. In order to ensure constant temperature on the opposite side of the glass slide, we place a sapphire disk, which has a much higher thermal conductivity than glass, between the slide and the heater. The thermal penetration depth in the glass during the entire impact process can be estimated to be $\sqrt{\alpha_{\mathrm{s}} \tau_{\text {imp }}} \approx 10 \mu \mathrm{m}$, well below the $1 \mathrm{~mm}$ thickness of the slide. Before the impact, the slide surface is set at a temperature $T_{\text {sur }, 0}$ from $80^{\circ} \mathrm{C}$ to $590^{\circ} \mathrm{C}$ by an electric heater; $T_{\text {sur }, 0}$ was measured by a surface probe before each experiment. In view of the importance of the surface roughness for the Leidenfrost temperature (and the splashing threshold [55]), we measured by atomic force microscopy the RMS roughness of the glass slide, finding it smaller than $10 \mathrm{~nm}$.

Next to the aforementioned side-view camera, we use two different imaging techniques to view the phenomena occurring during impact from below. In both techniques, the bottom view cameras (Photron SAX-2) were synchronized with the side view camera. The transparency of the setup enables us to use back light imaging through the substrate to obtain the bottom view (Fig. 5.1b). To measure the wetted area we use total internal reflection imaging (TIR), which was used previously in literature in both impact $[53,55]$ and boiling studies $[13,56]$. Here, the light is coupled into the glass slide by a dove prism and, when the surface is dry, reflects from the top surface, so that 
Table 5.1: Relevant properties of ethanol and glass, and for comparison sapphire

\begin{tabular}{|l|l|l|l|l|}
\hline Property & units & Ethanol & Glass & Sapphire \\
\hline $\begin{array}{l}\text { Density } \rho \\
\text { Latent heat } L\end{array}$ & {$\left[\mathrm{~kg} / \mathrm{m}^{3}\right]$} & 789 & 2520 & 3970 \\
$\begin{array}{l}\text { Specific heat } C p \\
\text { Surface tension } \sigma\end{array}$ & {$[\mathrm{kJ} / \mathrm{kg}]$} & 837 & & \\
$\begin{array}{l}{[\mathrm{kJ} / \mathrm{kg} \mathrm{K}]} \\
{[\mathrm{mN} / \mathrm{m}]}\end{array}$ & 2.4 & 0.8 & 0.8 \\
$\begin{array}{l}\text { Thermal conductivity } k \\
\text { Thermal diffusivity } \alpha\end{array}$ & $\begin{array}{l}{[\mathrm{W} / \mathrm{K} \mathrm{m}]} \\
{\left[\mathrm{m}^{2} / \mathrm{s}\right]}\end{array}$ & & 1 & 32 \\
$k \rho C p$ & {$\left[J^{2} / \mathrm{K}^{2} \mathrm{~s} \mathrm{~m}^{4}\right]$} & & $4 \times 10^{-7}$ & $1 \times 10^{-5}$ \\
& & & $2 \times 10^{6}$ & $1 \times 10^{8}$ \\
\hline
\end{tabular}

all incident light enters the camera. However, if the surface is wetted by the drop, the refractive index changes from 1 (air) to 1.4 (ethanol) and the light can exit the slide through the drop, resulting in a dark spot on the camera. Just before the drop contacts the slide, it traverses the thickness of the evanescent wave, resulting in loss of intensity on the camera. As a consequence of the Fresnel equations, the intensity decreases exponentially with the distance from the slide surface, $I(y) / I(\infty)=1-\exp (-\beta y)$, where $\beta=4 \pi /(\lambda n) \sqrt{\sin ^{2} \theta-n^{2}}$ [52]. In our case, $\beta=80 \mathrm{~nm}^{-1}$ depending on wavelength $\lambda$, refractive index ratio $n=n_{\mathrm{t}} / n_{\mathrm{i}}$ (with $t$ and $i$ indicate transmitted and incident) and the incident angle $\theta$ of the laser beam with the normal of the surface. This circumstance permits us to map the local height of the drop surface over the slide from the light intensity measured by each pixel within $12.5 \%$ uncertainty (see section 5 .B). We correct for light reflections from the droplet interface, according to Court and von Willisen [59]. By this TIR technique we can clearly distinguish whether or not portions of the drop under-surface are in direct contact with the slide. Using a long-distance microscope (Navitar 12x Telecentric variable zoom system) we obtained a field of view of $15 \mathrm{~mm}$ and $1 \mathrm{~mm}$ for the study of millimetric and microdrops respectively. The side view and bottom view are illuminated by a white light source, while for the TIR recordings a continuous red diode laser $(\lambda=643 \mathrm{~nm})$ is used. The glass slide is optically coupled to the prism and sapphire disk by silicon oil to allow the laser light to propagate through all materials. The frame rate for the TIR imaging was $40000 \mathrm{fps}$ and we used a shutter speed of $300 \mathrm{~ns}$ to prevent motion blurring. In the microdroplet case, the TIR frame rate was increased to $100000 \mathrm{fps}$ for higher temporal resolution.

\subsection{Results and discussion}

\subsubsection{Observations}

Figure 5.2 shows four examples of $2.6 \mathrm{~mm}$-diameter drops impacting the glass slide at various temperatures with a velocity of $U=1.3 \mathrm{~m} / \mathrm{s}(W e=1293)$. For each example, the sequence on the left shows the side view of the impact. The sequence on the right is a composite of the TIR recording (left half), which shows the wetted area, and the bottom view (right half). These images are recorded in different experiments. However, we are confident that both can be considered as showing essentially the same event due to the repeatability of the experiment and to the fact that both recordings are synchronised 
with the side view.

For impacts from room temperature (Fig. 5.2a) up to $186^{\circ} \mathrm{C}$ we observe the spreading of the drops on the solid surface to proceed at velocities similar to those reported in previous studies of the impact on cold surfaces $[44,65,80]$. The radius from the TIR data corresponds to that of the moving contact line, represented by a red circle, in both the bottom and side views. Heating the substrate further changes this correspondence: at $210^{\circ} \mathrm{C}$ we see that the growth of the wetted area is suppressed: the drop liquid is now partially levitating above the solid surface, forming a lamella which hovers over its own vapour (Fig. 5.2b). As the drop is now partly levitating and partly touching the solid, it is in a transitional state between contact and Leidenfrost boiling. With increasing temperature, the wetted area shrinks further (Fig. 5.2c) until at $280^{\circ} \mathrm{C}$ the surface is never wetted by the drop, which is thus in the Leidenfrost state (Fig. 5.2d). This sequence of behaviours with increasing temperature is sketched in Fig. 5.3a.

In the transition boiling regime, the TIR recordings show a fingering pattern (Fig. 5.2b and c), as reported for earlier experiments on glass [56]. However, preliminary results (not shown here) for impacts on a sapphire plate with a comparable smoothness do not exhibit this fingering pattern [73]. As sapphire is a better thermal conductor than glass, these observations suggest that the thermal properties of the substrate play a role in the appearance of this pattern.

As exemplified in Fig. 5.2, for each surface temperature $T_{\text {sur, } 0}$, the bottom views (rightmost images) provide information on the spreading front of the drop and on the wetted area. We define the radius $R_{\mathrm{S}}(t)$ of the spreading front as the radius of the dark circle marking the edge of the drop in the bottom view images. The radius $R_{\mathrm{W}}(t)$ of the wetted area is defined as the radius of the smallest circle encompassing the wetted area, as shown by the red circle in the figure. The data obtained in this way are shown in Fig. 5.3 for various surface temperatures up to the incipience of the Leidenfrost state. We find that $R_{\mathrm{S}}(t)$ (Fig. 5.3b) increases only slightly with increasing $T_{\mathrm{sur}, 0}$. At the lower temperatures, $R_{\mathrm{W}}(t)$ (Fig. 5.3c) keeps pace with $R_{\mathrm{S}}(t)$. However, from the start of the transition regime at $203^{\circ} \mathrm{C}$, this correspondence between the two quantities is lost and $R_{\mathrm{W}}(t)$ grows much less than $R_{\mathrm{S}}(t)$. At the end of the transition regime, $R_{\mathrm{W}}$ settles at about $0.75 D_{0}$. Above $266^{\circ} \mathrm{C}$, no wetting is found and the drops are in the Leidenfrost state. It is also noteworthy that a non-monotonic increase of $R_{\mathrm{S}}$ with $T_{\text {sur }, 0}$ is found in Fig. $5.3 \mathrm{~b}$ for $t / \tau_{\mathrm{imp}}>1$. This is caused by the lamella breakup which is strongest at the beginning of the transition boiling as shown in Fig. 5.2.

We performed experiments for different impact velocities as well. The observed boiling behaviours are plotted in the phase space $\left(U, T_{\text {sur }, 0}\right)$ in Fig. 5.4. Three main regions can be identified: for low substrate temperatures we find stable contact boiling, represented by blue circles. Here, the growth of the wetted area keeps pace with the spreading as for impact on unheated surfaces. In orange we indicate the aforementioned transition region where a lamella is ejected. Here, the wetting velocity of the contact line lags behind the expansion rate of the lamella. This regime is characterised by two different behaviours, indicated by $\triangle$ and $\diamond$, to be discussed later. For high temperatures we find no wetted area and the impacts are in the Leidenfrost state, shown as red $\square$. 


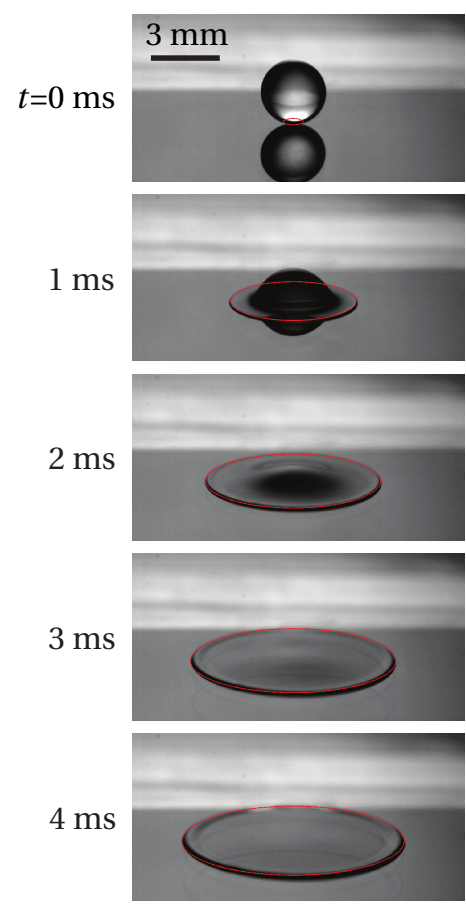

(a) $20^{\circ} \mathrm{C}$
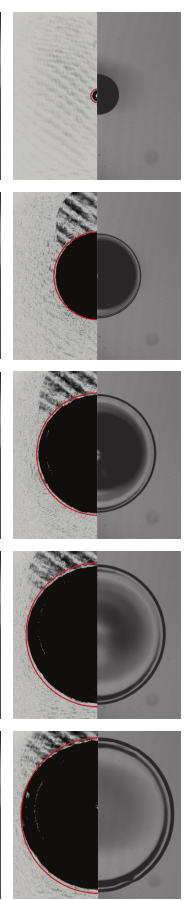
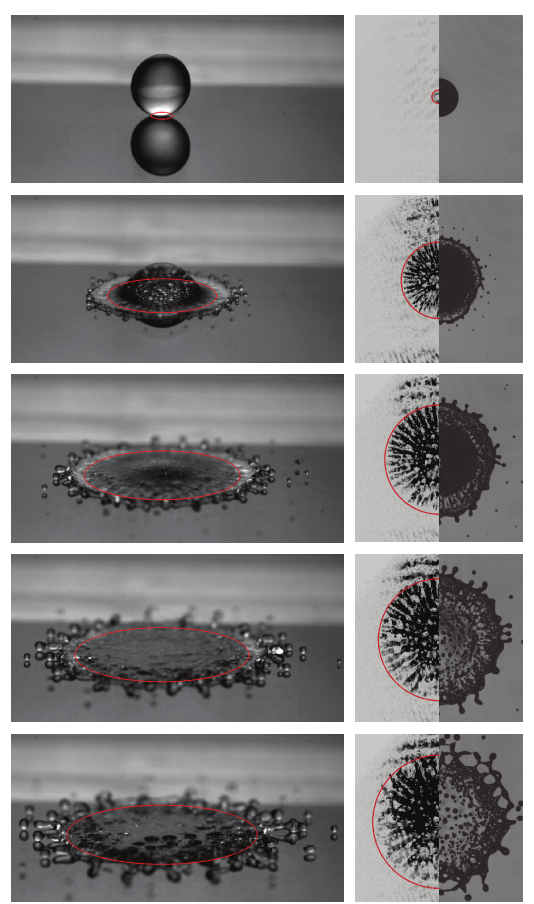

(b) $210^{\circ} \mathrm{C}$

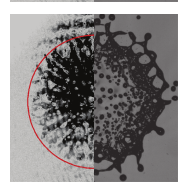



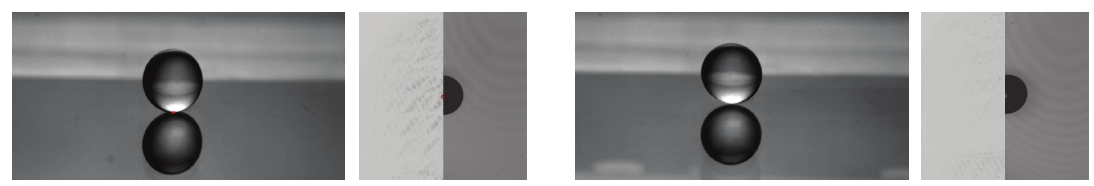

$t=0 \mathrm{~ms}$
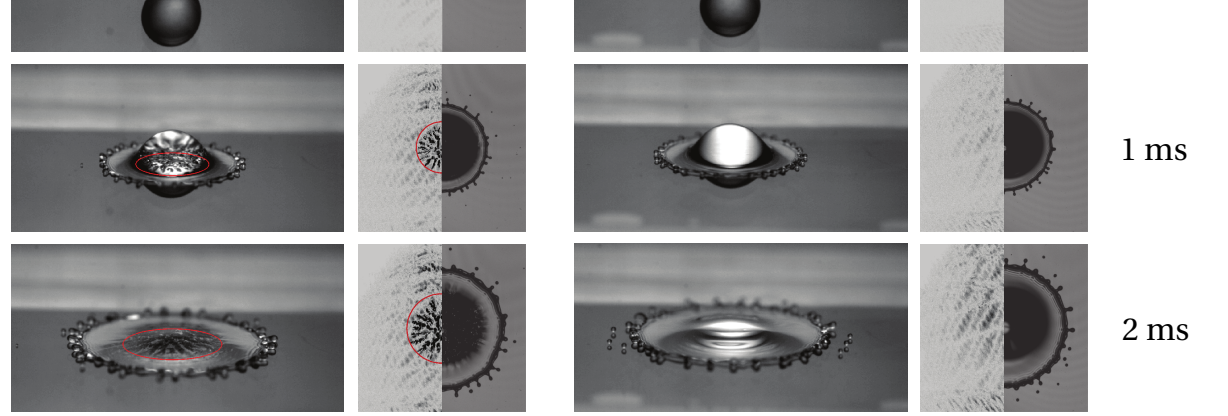

$1 \mathrm{~ms}$
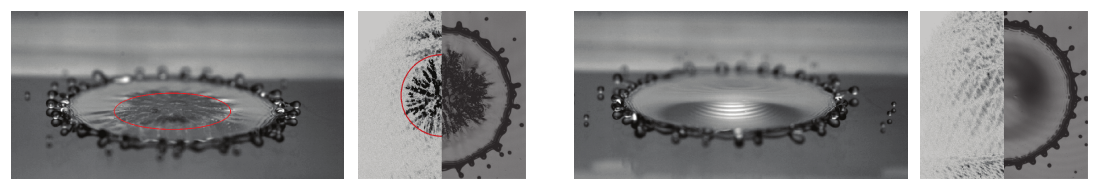

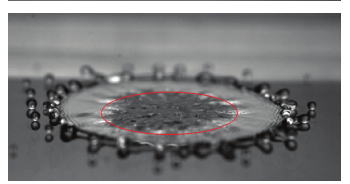

(c) $245^{\circ} \mathrm{C}$
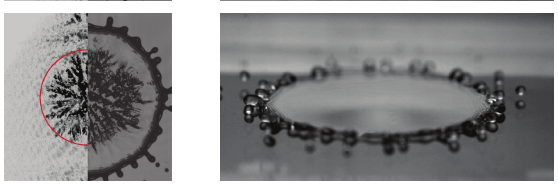

(d) $280^{\circ} \mathrm{C}$

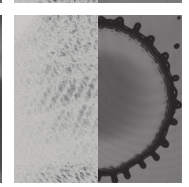

$4 \mathrm{~ms}$

Figure 5.2: Sequences of ethanol drops impacting on a glass substrate with velocity of $1.3 \mathrm{~m} / \mathrm{s}$. The substrate is at an initial temperature of $T_{\text {sur }, 0}=20$ (a), 210 (b), 245 (c) and $280^{\circ} \mathrm{C}(\mathrm{d})$. In each sequence, the left images in each pair show the side-view, while the right ones are a composite of the bottom view (right part) and the total internal reflection TIR (left part) recordings. The red circles in the side-view represent the measured wetted area from the TIR recordings. Sequence (a) shows a typical cold case, where all three recordings reveal the same wetted area. Impacts in the transition regime are shown in sequences (b) and (c), where the contact line spreading is suppressed (TIR), while a lamella spreads and fragments as visible in the bottom view. Sequence (d) shows an impact in the Leidenfrost regime, with no touch-down, which cannot be deduced from the bottom view but is revealed by the TIR. Some 'shadows' in the TIR images are artefacts of the optical system. The movies can be found online [57]. 
(a)

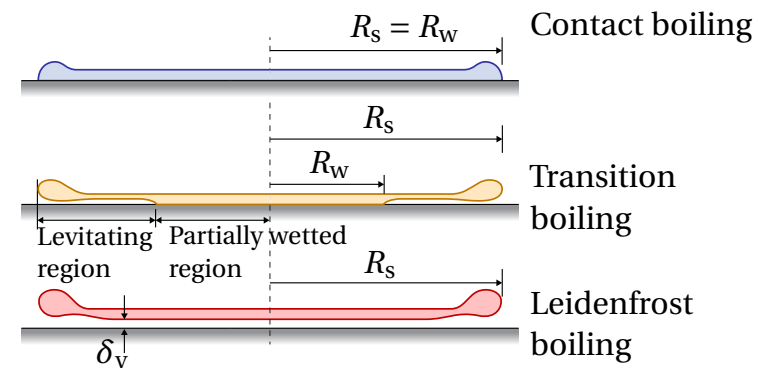

(b) 5
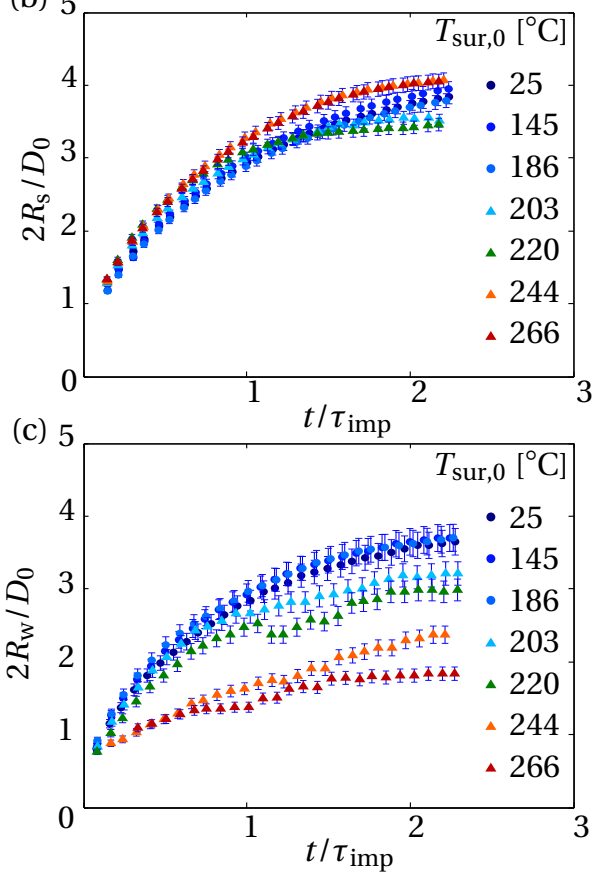

Figure 5.3: (a) Schematic of the three different spreading states: contact, transition and Leidenfrost boiling, indicating the difference between the radius of the wetted area $R_{\mathrm{W}}$ and spreading front $R_{\mathrm{S}}$ (within 5 and $10 \%$ uncertainty, see section 5.A). Observed time evolution of $R_{\mathrm{s}}(\mathrm{b})$ and $R_{\mathrm{w}}(\mathrm{c})$ of ethanol drops impacting at $U=1.3 \mathrm{~m} / \mathrm{s}$ for increasing surface temperatures. Data for cold and contact boiling is indicated by $\circ$. For contact boiling $R_{\mathrm{W}}=R_{\mathrm{S}}$, while the lamella speed increases in the transition regime $\triangle$; the wetting speed instead decreases, as compared to $\circ$. Later $(t=2 \mathrm{~ms})$, the lamella spreading stalls due to fragmentation (see Fig. 5.2b). The absence of wetting above $266^{\circ} \mathrm{C}$ shows the drops to be in the Leidenfrost state. 


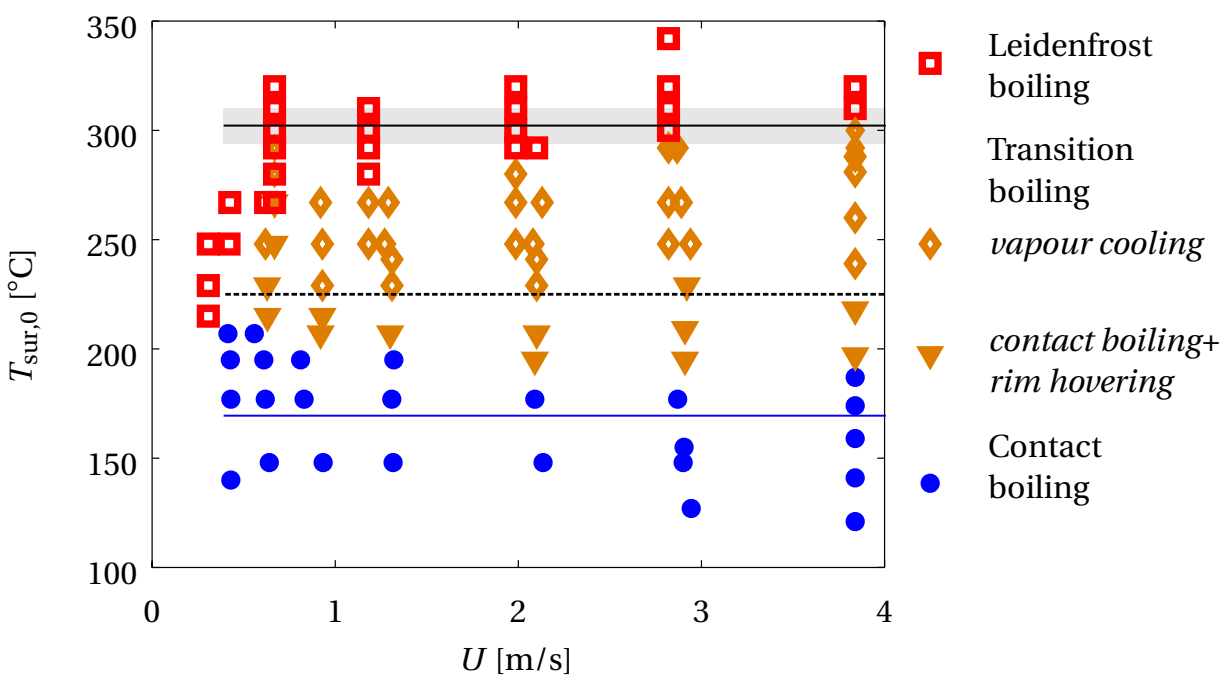

Figure 5.4: Phase space with an indication of the different boiling behaviours. The dashed line is a guide to the eye, indicating the boundary between contact boiling + rim hovering and vapour cooling. The solid black line denotes the transition to the Leidenfrost state, according to Equation 5.2; the shaded area indicates the estimated error. The Leidenfrost transition for isothermal impacts on sapphire is shown for comparison by the blue line at $T_{\mathrm{sur}, 0}=170^{\circ} \mathrm{C}$ (data from H.J.J. Staat et al. [69]).

\subsubsection{Subcooling effect causes touch-down}

The points denoted by $\triangle$ in the phase space of Fig. 5.4 indicate drops that, even though partially levitated, immediately exhibit stable boiling on the wetted area; this regime is indicated as contact boiling + rim hovering in the legend of the figure. This behaviour is strikingly different from that of drops that levitate at the very beginning of the impact, but then touch down at a later time. This regime, to which we refer as vapour cooling, is marked by $\diamond$ in Fig. 5.4. A detailed example of the drop behaviour in this case is shown in Fig. 5.5; the drop diameter is $2.3 \mathrm{~mm}$, the impact velocity $U=3.84 \mathrm{~m} / \mathrm{s}$ and the glass slide temperature $T_{\text {sur }, 0}$ is $292^{\circ} \mathrm{C}$. For each frame in this figure, the upper half shows the TIR image, while the lower half is the reconstructed height profile of the underside of the drop in tens of nm resolution. Here the drop first remains levitated (i-iv), but in frames (v) and (vi) it starts to randomly touch the surface, exhibiting what may be called unstable boiling. This behaviour is different from what we earlier referred to as stable boiling in which the liquid-solid contact does not fluctuate appreciably. Later, the drop wets the surface and a stable fingering pattern is observed, as discussed before. It is also interesting to note that no drop is visible in frame (ii), although it reappears in frame (iii). This behaviour implies that the pressure under the drop is initially so large as to briefly push it back. We have encountered this phenomenon only in the upper range of the temperatures investigated.

This delayed touch-down mechanism is due to the cooling that the vapour generation induces in the solid. Due to the small thermal conductivity of glass, $T_{\text {sur }}(t)$ drops 
significantly during the early stages of the impact to such an extent that the drop levitation cannot be maintained. Such effects of the solid thermal properties are frequently encountered in heat transfer phenomena. Examples are the influence of the thermal conductivity of a hot metal being wetted by the advancing front of a cold liquid [88, 89], the similar effect of the substrate influencing the waiting time between two successive bubbles in boiling [90] and the reduction of the Nusselt number in turbulent thermal convection [91].

The situation that is established during the drop impact is characterised by the very short distance (less than $1 \mu \mathrm{m}$ ) that separates the liquid surface from the solid and a much longer scale parallel to the solid surface. This large scale separation justifies the use of a one-dimensional conduction model in the solid. We assume the drop surface to remain at the saturation temperature $T_{\mathrm{b}}$ and model the heat flux out of the solid as $h \cdot\left(T_{\text {sur }}(t)-T_{\mathrm{b}}\right)$, where $h$ is a heat transfer coefficient. We may expect $h$ to be enhanced beyond the pure conduction value $\left(k_{\mathrm{v}} / \delta_{\mathrm{v}}\right.$, with $k_{\mathrm{v}}$ the thermal conductivity of the vapour and $\delta_{\mathrm{v}}$ the thickness of the vapour layer) by the vapour flow between the drop and the surface, although the order of magnitude should not be altered. This expectation is based on the well known relation of the Nusselt number for fully developed laminar flow in ducts [1]. The solution of the conduction problem thus posed is well known $[10,36,72,92]$. The temperature of the solid surface $T_{\text {sur }}(t)$ is found to be given by:

$$
\frac{T_{\text {sur }}(t)-T_{\mathrm{b}}}{T_{\text {sur }, 0}-T_{\mathrm{b}}}=\exp \left(\frac{t}{\tau_{\text {th }}}\right) \operatorname{erfc}\left(\sqrt{\frac{t}{\tau_{\text {th }}}}\right),
$$

in which the characteristic thermal time scale is given by $\tau_{\text {th }}=k_{\mathrm{s}} \rho_{\mathrm{s}} C p_{\mathrm{s}} h^{-2}$.

The thermal time scale depends on the heat transfer coefficient $h$, which is unknown for our system. Therefore, in order to determine $\tau_{\text {th }}$, we measure for each experiment the cool-down time $t_{\mathrm{cd}}$, i.e., the time before stable contact boiling is observed. Then we apply Equation 5.1 to fit our data and obtain $\tau_{\text {th }}$ from which we calculate $h$. The cooldown time $t_{\mathrm{cd}}$ is obtained by studying the temporal correlation coefficient between image pairs. This coefficient is a measure of the degree of similarity between two successive frames. For $t<t_{\mathrm{cd}}$ the drop is either still levitating or randomly touching the substrate. The wetted area is then rapidly varying and the resulting correlation between successive images is poor. When the surface is cooled down by the evaporation, on the other hand, the wetted patches become more stable, resulting in a high correlation coefficient.

We used sequences such as that of Fig. 5.5 to create a time series of correlation coefficients, an example of which is shown in Fig. 5.6a. Here, the sudden increase in correlation is readily identifiable as occurring for $t \approx 0.7 \mathrm{~ms}$, where two straight lines fitted to the correlation data intersect. Skipping frames (inset) reveals that the technique is fairly insensitive to the frame rate (provided, of course, it is not too short). We apply this technique to measure $t_{\mathrm{cd}}$ for various impact velocities and initial plate temperatures $T_{\text {sur, } 0}$. The results are presented in Fig. 5.6c and show that, for higher temperatures, it takes more time for the surface to cool down to the temperature level required for stable boiling. Furthermore, the data for different impact velocities exhibit a near-collapse. This is in agreement with the dashed line in Fig. 5.4 being independent of velocity as well. Both observations indicate that the cooldown time is at most weakly dependent on 


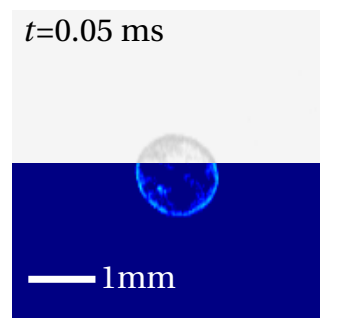

(i)

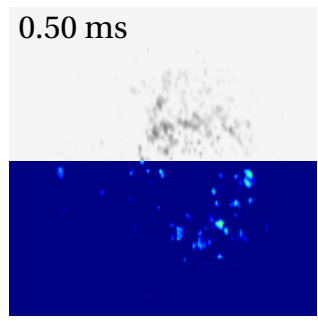

(iv)

$0.95 \mathrm{~ms}$

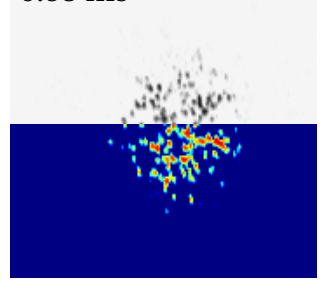

(vii)
$0.20 \mathrm{~ms}$

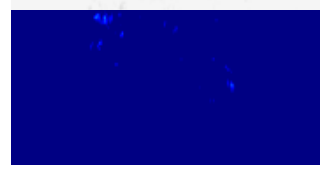

(ii)

\section{$0.65 \mathrm{~ms}$}

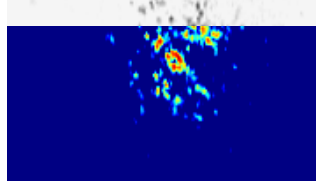

(v)

\section{$1.10 \mathrm{~ms}$}

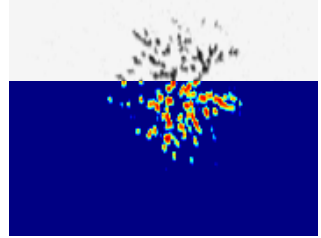

(viii)

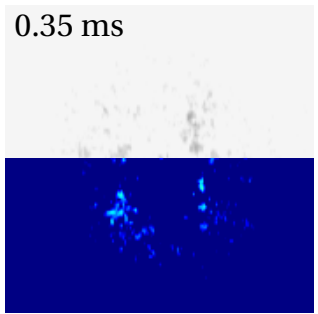

(iii)

$0.80 \mathrm{~ms}$

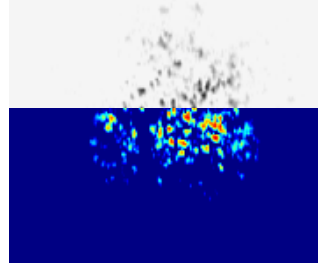

(vi)

$1.25 \mathrm{~ms}$

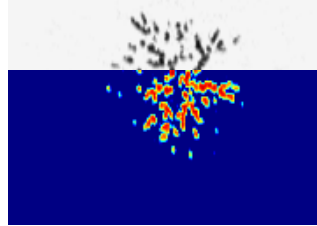

(ix)

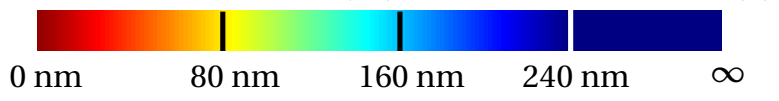

Figure 5.5: TIR data of an ethanol drop, with a diameter of $2.3 \mathrm{~mm}$, impacting at $U=$ $3.8 \mathrm{~m} / \mathrm{s}$ a heated glass surface of $T_{\mathrm{sur}, 0}=292^{\circ} \mathrm{C}$. The upper half of each frame shows the measured light intensity. As explained in the text, from these data we deduce the local height of the drop surface above the solid, i.e., the local thickness of the vapour layer, which is shown in the lower half (with $9 \%$ uncertainty, see section 5.B). The sequence shows the change from levitation (i-iv) to unstable contact (v,vi) to eventually stable boiling (vii-ix). The absence of the signal in (ii) results from a brief upward motion of the drop surface, only observed for high surface temperatures. 


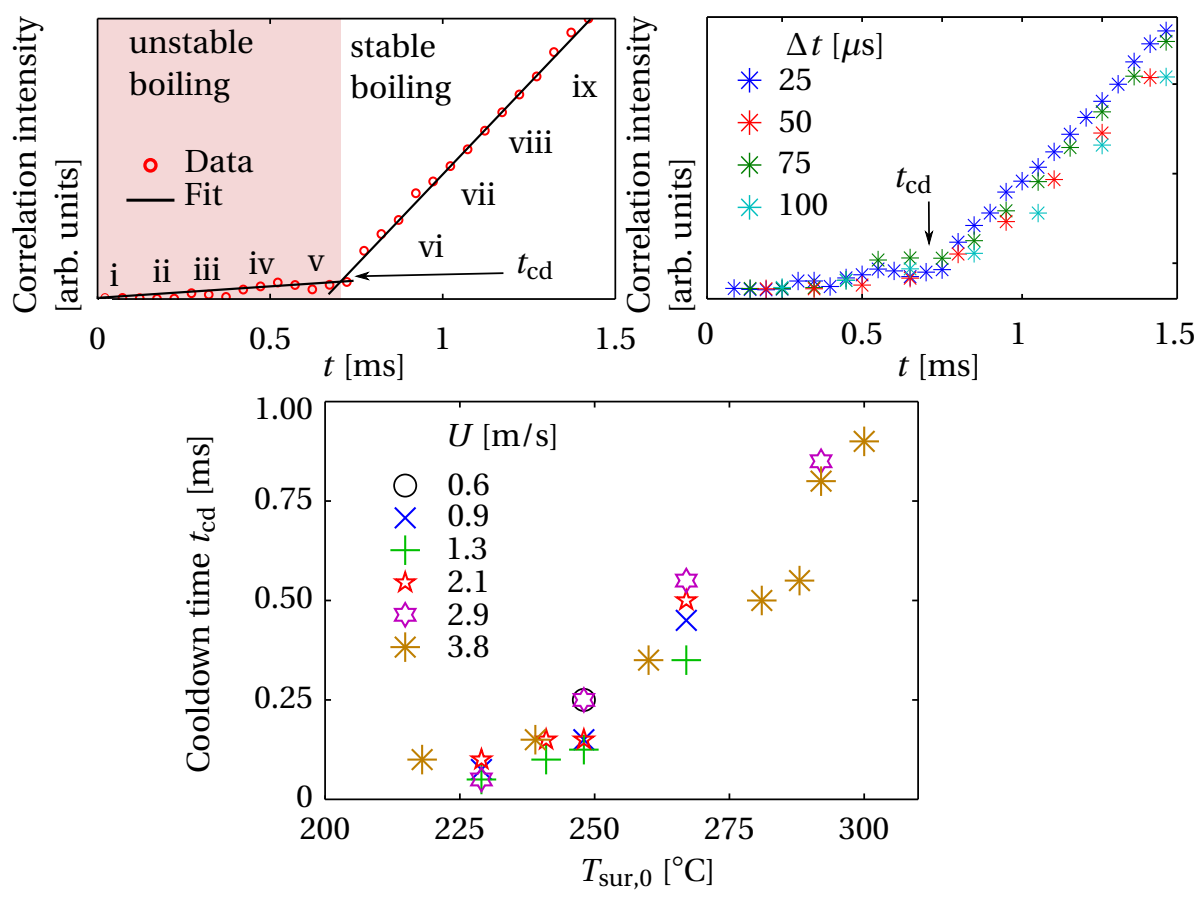

Figure 5.6: (a): Time series of the correlation coefficient for the data shown in Fig. 5.5 with matching roman numerals. Figure (b) shows the effect of correlating pairs of nonconsecutive images separated by 1 to 3 frames. (c): Measured cool-down times (defined in the text) for various impact velocities as a function of initial substrate temperature. For clarity, only one set of error bars is shown.

the impact velocity. However we do observe for high initial surface temperatures only stable boiling for high impact velocities. This is due to the higher dynamic pressure, forcing the drop more strongly towards the surface [44,93].

Once $t_{\mathrm{cd}}$ has been determined, we can proceed to use the information to estimate the thermal time scale $\tau_{\text {th }}$ appearing in Equation 5.1. Since for $t=t_{\mathrm{cd}}$ the surface temperature becomes too low to support the drop we deduce that, at this instant, the surface temperature equals the static Leidenfrost temperature for isothermal surfaces, i.e., $T_{\text {sur }}\left(t=t_{\mathrm{cd}}\right)=160^{\circ} \mathrm{C}$ [10]. We plot in Fig. 5.7 for each experiment $\left(160^{\circ} \mathrm{C}-T_{\mathrm{b}}\right) /\left(T_{\mathrm{sur}, 0}-T_{\mathrm{b}}\right)$ against $t_{\mathrm{cd}}$. The black lines correspond to various solutions of Equation 5.1 and the best fit of the data is the blue line with $\tau_{\text {th }}=0.3 \mathrm{~ms}$. The fit is not very sensitive in this range either to the precise value of $t_{\mathrm{cd}}$ or to the value assumed for the static isothermal Leidenfrost temperature. Although the fit is reasonably close to the data, there seems to be some difference between large and small values of $t_{\mathrm{cd}}$, which corresponds to a slight increase of the thermal time scale with $t_{\mathrm{cd}}$ and, therefore, with $T_{\text {sur }, 0}$. This results implies that the average heat transfer coefficient decreases somewhat with increasing surface temperature. Possible causes can be a larger initial vapour layer thickness as would be implied by the behaviour described before in connection with 


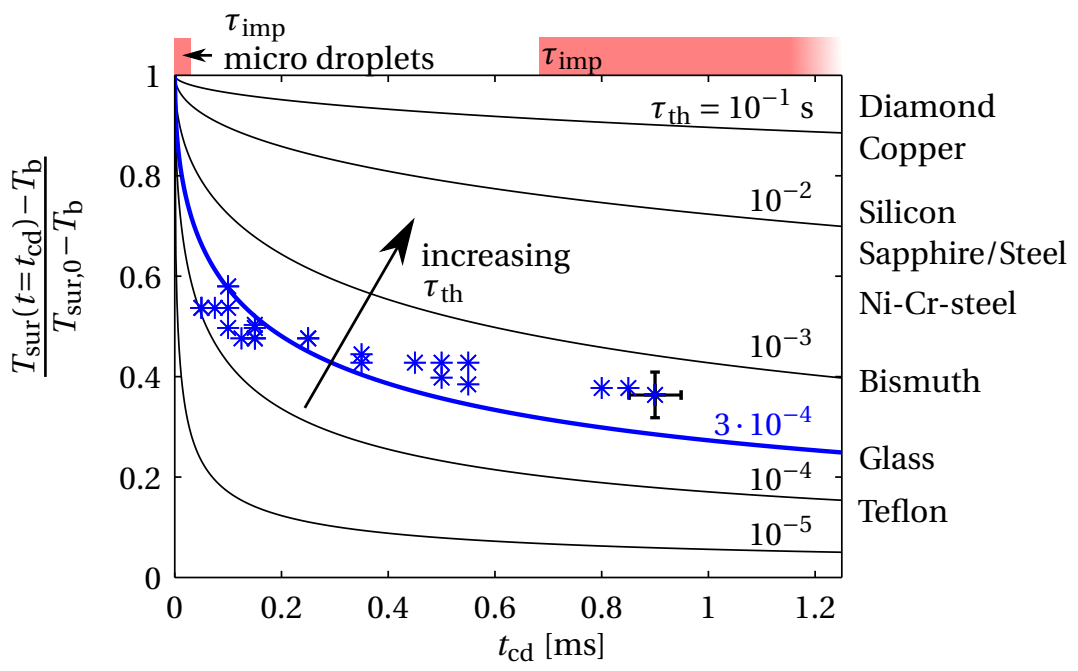

Figure 5.7: As argued in the text, for $t=t_{\mathrm{cd}}$, the surface temperature $T_{\mathrm{sur}}\left(t_{\mathrm{cd}}\right)$ should equal $160^{\circ} \mathrm{C}$. The asterisks show the value $\left(T_{\text {sur }}\left(t_{\mathrm{cd}}\right)-T_{\mathrm{b}}\right) /\left(T_{\mathrm{sur}, 0}-T_{\mathrm{b}}\right)$ for various values of $T_{\mathrm{sur}, 0}$, in correspondence of the $t_{\mathrm{cd}}$ measured in each experiment, as presented in Fig. 5.6c. For clarity, only one set of error bars is shown. The best fit of the data by Equation 5.1 is presented by the thick blue line; the remaining black lines present other solution corresponding with different thermal time scales. These solutions match various materials listed on the right, assuming that for impacts on smooth materials the heat transfer coefficient through the vapour layer has the constant value measured in the present study (here $8 \times 10^{4} \mathrm{~W} /\left(\mathrm{Km}^{2}\right)$ ). The red areas at the top of the figure indicate the impact time scales examined here for micro- and millimetric drops.

frame (ii) of Fig. 5.5 and increased convection cooling by the vapour.

It is interesting to compare the thermal time scale $\tau_{\text {th }}$ with the time $\tau_{\text {imp }}=D_{0} / U$ characteristic of the impact, by which all the downward momentum is converted into the radial direction and the dynamic pressure vanishes [71]. This is also the longest time the residual momentum can maintain the drop against the hot surface. Thus, if $\tau_{\text {th }}$ is equal or smaller than $\tau_{\text {imp }}$, the surface will cool down significantly. This is the case for all the impacts of millimetric drops studied here, for instance: in Fig. $5.5 \tau_{\text {imp }}=0.6 \mathrm{~ms}$ which is approximately $\tau_{\mathrm{th}}$.

From the thermal time scale $\tau_{\text {th }}$ we can deduce an estimate for the heat transfer coefficient $h=\sqrt{\left(k_{\mathrm{s}} \rho_{\mathrm{s}} C p_{\mathrm{s}} / \tau_{\mathrm{th}}\right)}$, which is therefore found to be $8 \times 10^{4} \mathrm{~W} /\left(\mathrm{Km}^{2}\right)$. For pure conduction through the vapour layer, this result corresponds to a thickness $\delta_{\mathrm{v}} \approx$ $100 \mathrm{~nm}$. This estimate is in agreement with our measurements of the minimum vapour thickness (for example Fig. 5.5) obtained from the evanescence wave attenuation length. Furthermore, assuming that this thickness is typical for impacts on smooth surfaces, one can now predict whether or not an impact can be considered as isothermal: the impact conditions yield $\tau_{\text {imp }}$ and one estimates $\tau_{\text {th }}=\left(100 \mathrm{~nm} / k_{\mathrm{v}}\right)^{2} k_{\mathrm{s}} \rho_{\mathrm{s}} C p_{\mathrm{s}}$ from the surface properties. The surface cooling for various materials based on their estimated $\tau_{\text {th }}$ is shown in Fig. 5.7. 
Table 5.2: Observed residence time of the drop within $100 \mathrm{~nm}$ from the solid surface for impact on a glass slide. The last column is the estimated Leidenfrost temperature obtained similarly to (5.2), using for the residence time the value listed in column four in place of $0.51 \mathrm{~ms}$.

\begin{tabular}{|l|l|l|l|r|}
\hline$U[\mathrm{~m} / \mathrm{s}]$ & $\begin{array}{l}W e= \\
\rho_{\mathrm{l}} U^{2} D_{0} /\left(2 \sigma_{1}\right)\end{array}$ & $\begin{array}{l}\text { Lowest } T_{\mathrm{L}} \\
{\left[{ }^{\circ} \mathrm{C}\right]}\end{array}$ & $\begin{array}{l}\text { Residence } \\
\text { time }[\mathrm{ms}]\end{array}$ & $\begin{array}{c}T_{\mathrm{L}} \text { by } \\
\text { Eq. } 5.2\left[{ }^{\circ} \mathrm{C}\right]\end{array}$ \\
\hline 0.4 & 8 & 267 & 0.4 & $285 \pm 10$ \\
0.6 & 27 & 280 & 0.45 & $294 \pm 10$ \\
1.3 & 111 & 280 & 0.6 & $318 \pm 10$ \\
2.1 & 279 & 290 & 0.55 & $310 \pm 10$ \\
2.9 & 538 & 300 & 0.5 & $303 \pm 10$ \\
3.8 & 740 & 310 & 0.6 & $318 \pm 10$ \\
\hline
\end{tabular}

Focussing in more detail on the phase space of Fig. 5.4, we now aim to predict the boundary between the transition region (orange) and the Leidenfrost region (red). For all impact velocities we select the lowest surface temperature resulting in the Leidenfrost state and measure the time that the drop is within $100 \mathrm{~nm}$ distance of the substrate, see Tab. 5.2. The average time observed is $0.51 \mathrm{~ms}$ without any significant velocity dependence, in accordance with our previous observations (Fig. 5.6c). This 'residence time' is typically shorter than $\tau_{\text {imp }}$. Using these observations, we can calculate $T_{\text {sur, } 0}$ by solving Equation 5.1 with $T_{\text {sur }}(t=0.51 \mathrm{~ms})=160^{\circ} \mathrm{C}$, the static isothermal $T_{\mathrm{L}}$ for ethanol:

$$
T_{\text {sur }, 0}=\frac{160^{\circ} \mathrm{C}-80^{\circ} \mathrm{C}}{\exp (0.51 / 0.3) \operatorname{erfc}(\sqrt{0.51 / 0.3})}+80^{\circ} \mathrm{C}=304^{\circ} \mathrm{C} \text {. }
$$

The obtained prediction is plotted in Fig. 5.4 by a black solid line; the shaded area indicates the estimated error. We see that including this correction for the non-isothermal behaviour of the solid yields a good prediction for the dynamic Leidenfrost temperature.

\subsubsection{Micro droplets show isothermal behaviour}

The previous analysis assumed the elevated Leidenfrost temperature found for impacts on a glass surface to originate from the thermal properties of the substrate. In order to strengthen this conclusion, we also studied the case in which $\tau_{\text {imp }} \ll \tau_{\text {th }}$ by considerably shortening the impact time scale $\tau_{\text {imp }}$. This objective is achieved by using microdroplets, as the smaller diameter $D_{0}=300 \mu$ m lowers $\tau_{\text {imp }}=D_{0} / U$ to $30 \mu \mathrm{s}$, satisfying $\tau_{\text {imp }} \ll$ $\tau_{\text {th }}$. In this case we expect even a glass substrate to behave as isothermal with no observable cool-down effect. A sequence showing the full impact and spreading phases of a microdrop impacting at $U=8.9 \mathrm{~m} / \mathrm{s}$ a glass surface with $T_{\text {sur }, 0}=265^{\circ} \mathrm{C}$ (just below $T_{\mathrm{L}}$ ) is shown in Fig. 5.8.

Comparing the results with the millimetric drops shown in Fig. 5.5, we first observe a levitating droplet as well. From $t=50 \mu \mathrm{s}$ on, the drop touches the surface randomly. However, in contrast with the millimetric case, no stable boiling is observed for the microdroplet impact. Varying the impact velocity between 2 and $8.9 \mathrm{~m} / \mathrm{s}$, we did not observe any cool-down effect. In this case, the touch-down mechanism then is purely governed by the impact dynamics, as expected from the estimate of the time scales. We 


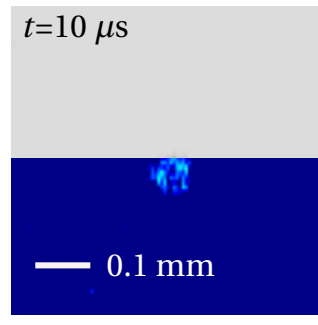

(i)

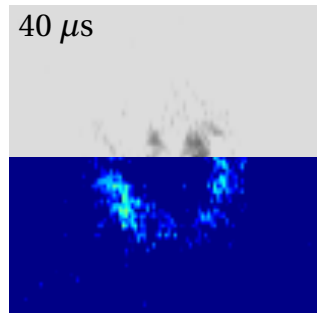

(iv)

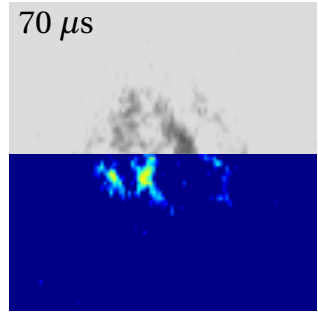

(vii)

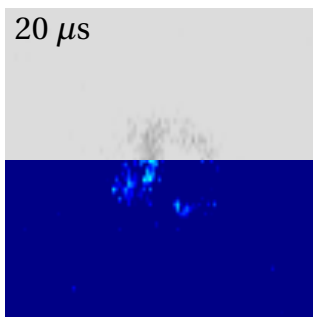

(ii)

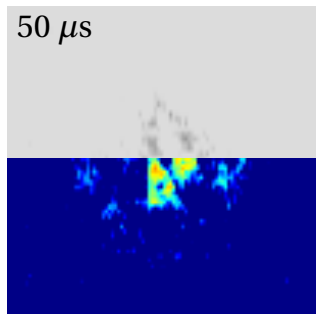

(v)

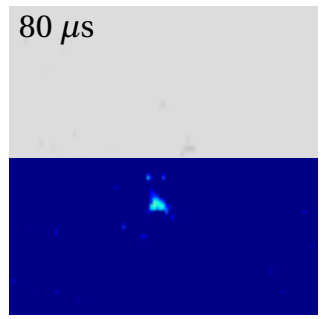

(viii)

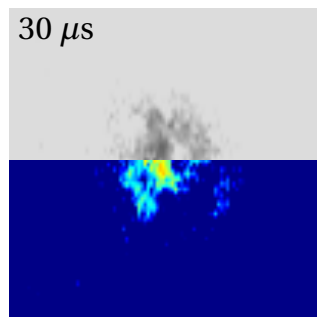

(iii)

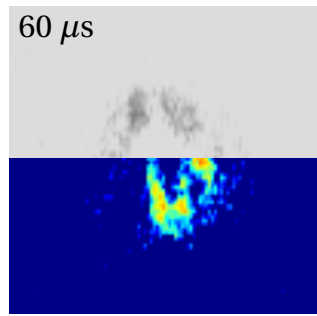

(vi)

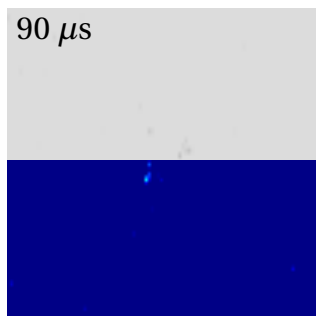

(ix)

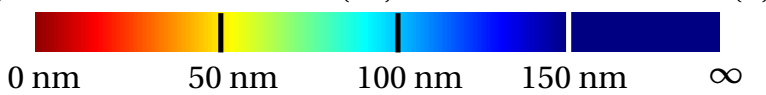

Figure 5.8: Similarly to Fig. 5.5, each frame is a composite of the TIR-measured light intensity and the corresponding thickness (with $12.5 \%$ uncertainty, see section 5.B) of the vapour film for an $300 \mu \mathrm{m}$-diameter ethanol droplet impacting a glass substrate just below the Leidenfrost temperature $T_{\text {sur }, 0}=265^{\circ} \mathrm{C}$. The impact velocity is $8.9 \mathrm{~m} / \mathrm{s}$ ( $W e=1874$ ). No stable boiling is observed as $\tau_{\mathrm{imp}} \approx 30 \mu \mathrm{s}$, which is much shorter than $\tau_{\text {th }}$. 
conclude therefore that the same surface that exhibits cooling with large drops, behaves as isothermal when the time scale of the impact dynamics is shorter than the thermal time scale.

\subsection{Conclusions}

We studied the impact of ethanol drops on superheated smooth glass substrates. We used high speed total internal reflection imaging to study the dynamics of the wetted area of the surface. We observed a new touch-down mechanism for millimetric drops caused by the cooling of the solid surface. By comparing the time scale $\tau_{\text {th }}$ for the cooling of the glass surface with the impact time scale $\tau_{\text {imp }}=D_{0} / U$ we concluded that a millimetric drop causes a significant cooling the glass surface. While the drop remains levitated during the early stages of the impact, this cooling causes it to eventually touch the solid. By means of a one-dimensional conduction model, we developed an estimate of the dynamic Leidenfrost temperature for a poorly conducting solid. We calculated $\tau_{\text {th }}$ for glass to be $0.3 \mathrm{~ms}$, which is well below $\tau_{\text {imp }}$ for the impact events studied here. For impact velocities in the range of 0.6 to $3.8 \mathrm{~m} / \mathrm{s}$, investigated in this study, the vapour thickness calculated from $\tau_{\text {th }}$ is approximately $100 \mathrm{~nm}$, consistent with our measurements. Using this information, together with the substrate properties one can thus a priori estimate the thermal time scale.

The previous conclusions are consistent with the results of another set of experiments on the impact of micro-droplets for which $\tau_{\text {imp }}$ is much shorter than $\tau_{\text {th }}$. In this case, indeed, we do not observe any cooling effect and the surface remains essentially isothermal.

To summarise, therefore, for $\tau_{\mathrm{imp}} \ll \tau_{\mathrm{th}}$, the solid remains essentially isothermal and the touch-down of the drop is governed by the dynamic pressure of the impact. For $\tau_{\text {imp }} \geq \tau_{\text {th }}$, on the other hand, the substrate is cooled during the spreading of the drop, which eventually touches the solid surface.

\section{Acknowledgements}

This work was partially supported by Fundamenteel Onderzoek der Materie, NanoNextNL and by an ERC-Advanced Grant. 


\section{A Image Processing}

The three types of recordings we obtain in our experiments are processed in different ways, using Matlab programming [62]. The side-view images are used for the measurements of the diameter and impact velocity by a simple edge detection technique. The classification of the boiling regimes are done manually by comparing all three image types by characteristics as explained in details in subsection 5.3.1.

The image analysis of the TIR data consists mainly of two processes: firstly, the image transformation and secondly the detection of the wetted area, as shown in Fig. 5.9 (a-f). Even though a droplet wets the substrate in an axisymmetric manner, the TIR image gives an ellipsoid as shown in Fig. 5.9a, due to the optical transformations in the setup. The original image is first binarized after background subtraction (b). From the binary image, the angle of rotation and aspect ratio are measured; for this measurement, the frame corresponding to $t / \tau_{\text {imp }}=0.25$ of room temperature experiment was typically used. Using the linear transformation, the circular droplet (c), is reconstructed.

The linear transformation obtained from the room temperature experiment was also used in the analysis of different temperature cases. A typical example of impact on a heated surface is shown in Fig. 5.9d-f, where the initially ellipsoidal fingering pattern in (d) is well transformed back to the circular one in (f). Note here that the envelope circle shown as the red line in (f) was determined from the average-intensity profile, such as shown in (g), as the point of abrupt decrease in mean value (solid line) and standard deviation (shaded area); the intensity profile was calculated from intensities along 50 to 100 lines spreading radially outward from the droplet centre, see Fig. 5.9e.

The radius of spreading front $R_{\mathrm{S}}$ was also determined from the bottom-view image as in the same manner for TIR analysis except for the linear transformation. We set the thresholding condition in such a way that the foot of the fingers at the rim of the droplet was detected as shown by the red circle in Fig. 5.9h.

The main cause of the uncertainty of the radius of the spreading front $R_{\mathrm{s}}$ arises from the non-uniform growth rate of the fingers pinched off from the peripheral rim; such non-uniform behaviour result in an uncertainty for defining the spreading front from the image intensity profile, a typical example of which is shown by the arrow in Fig. 5.9g. Similarly, for the radius of the wetting area $R_{\mathrm{W}}$, the non-uniform wetting rate of the fingering pattern causes the largest uncertainty. In summary, the uncertainty is, at most, $5 \%$ for $R_{\mathrm{S}}$ and $10 \%$ for $R_{\mathrm{W}}$.

\section{B Error estimate of the measured height from TIR mea- surement}

Starting from the equation for the height $h$ and the normalised intensity [59]: $I^{\prime}=\frac{I}{I_{\infty}}=$ $E E^{*}$, with $E$ being the electric field and $E^{*}$ its complex conjugate.

$$
\begin{aligned}
h & =-\beta \ln \left(\frac{r_{12}-E}{r_{32}-r r_{12} r_{32}}\right), \\
\text { where } \beta & =\frac{\lambda n}{4 \pi} \frac{1}{\sqrt{\sin \left(\theta_{\mathrm{i}}\right)^{2}-n^{2}}}
\end{aligned}
$$



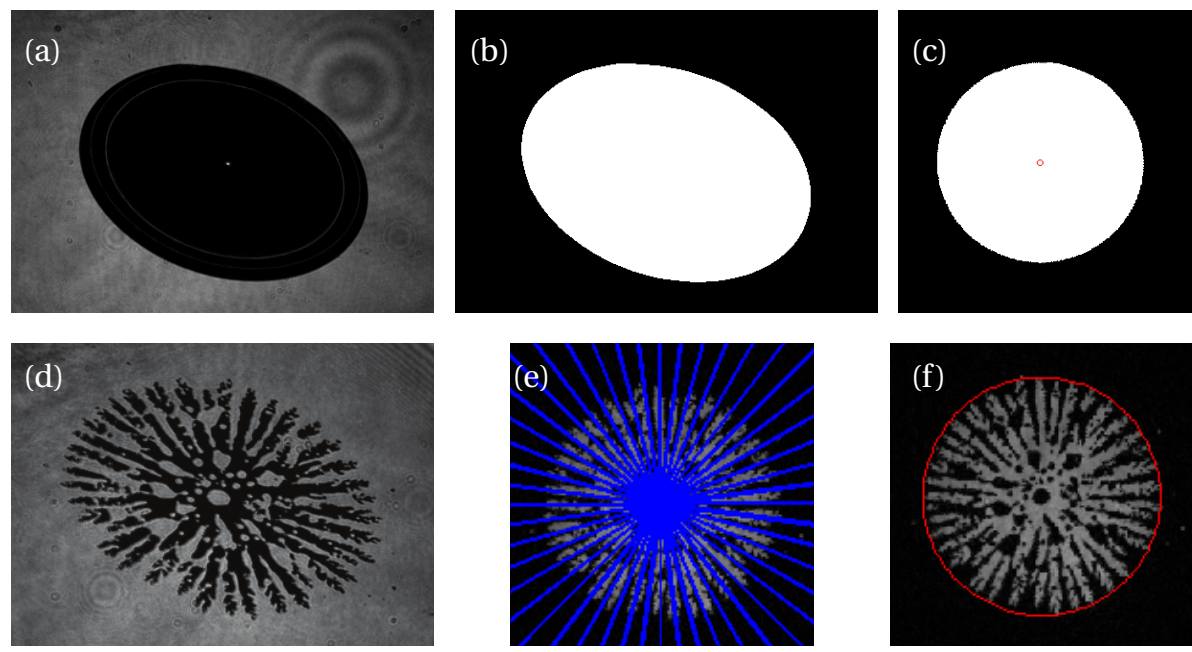

(g)
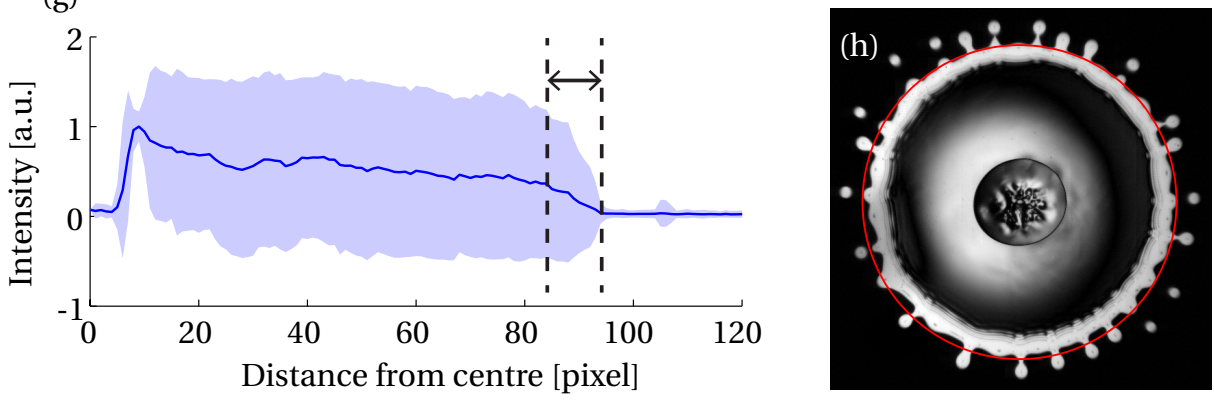

Figure 5.9: Typical examples of (a-c) images used for calibration of TIR image transform, (d-f) TIR images in transition boiling regime showing the transformation with the rate given in (a-c), (g) average intensity (solid line) together with standard deviation (shaded area) along the lines radially spread outward from the centre of wetted area (blue lines in (e)), and (h) an image of bottom view with backlighting after the background subtraction. The red circles in (f) and (h) show the detected wetting and spreading front, respectively. The uncertainty caused by the non-uniform growth rate of the fingers is shown by the arrow in (g). 
(a)

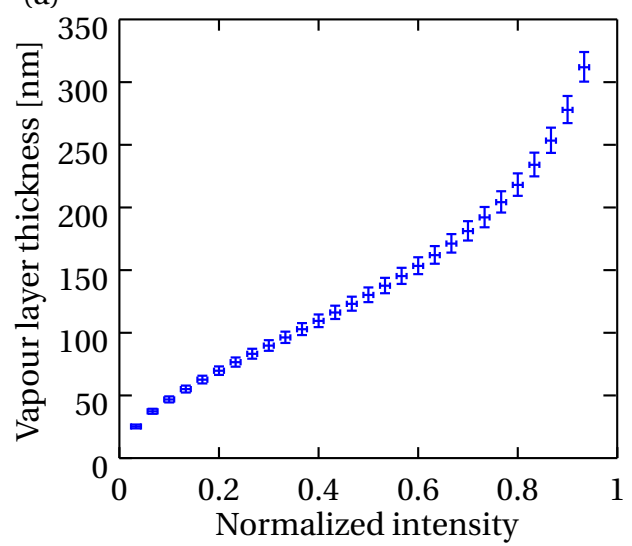

(b)

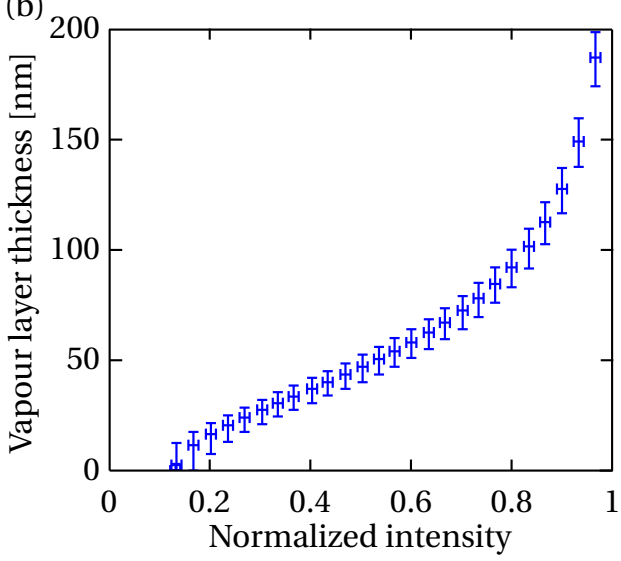

Figure 5.10: Error estimate for the millimetric (a) and micro droplet (b) measurements. The vertical errorbars the combined uncertainties in refractive indices, laser wavelength and angle of incidence of the laser.

Here $\lambda$ is $643 \mathrm{~nm}$, the wavelength of the light source, $n=1 / 1.51$ the ratio of refractive indices of the air and glass and $\theta_{\mathrm{i}}$ is the angle between the laser light and the normal of the glass surface, resulting in $\beta=81$ and $59 \mathrm{~nm}^{-1}$, with $\theta_{\mathrm{i}}=52^{\circ}$ and $61^{\circ}$, for the millimetric drops and micro droplet measurements respectively. The functions $r_{12}$ and $r_{32}$ are the reflectivity coefficients as given by the Fresnel equations for the solid-air and air-liquid interface for $\mathrm{p}$-polarized light respectively . These functions depend on $n, \theta_{\mathrm{i}}$ and $n_{\mathrm{d}}$, the refractive index of the droplet liquid. We then calculate numerically the error which is presented in 5.10:

Here the following uncertainties are used to estimate the total error: $\Delta \lambda=0.5$ $\mathrm{nm}, \Delta n_{\mathrm{ti}}=0.001$ where the refractive index was evaluated from room temperature to $400{ }^{\circ} \mathrm{C}$ [94], and $\Delta \theta_{\mathrm{i}}=0.5^{\circ}$ from our analysis uncertainty. We estimate the error in the measured intensity to be $2 \%$ originating from fluctuations in the laser intensity. From this analysis, we can approximate the error $\Delta h$ to increases linearly with height. The average relative error $\Delta h / h$ is $9 \%$ for millimetric drops and $12.5 \%$ for the micro droplet measurements. 
72 CHAPTER 5. COOLING EFFECTS IN HOT SUBSTRATES DURING DROP IMPACT 


\section{Chapter 6}

\section{Origin of spray formation during impact on heated surfaces ${ }^{\dagger}$}

In many applications it is crucial to control the heat transfer rate of impacting drops on a heated plate. When the solid exceeds the so-called Leidenfrost temperature, an impacting drop is prevented from contacting the plate by its own evaporation. As the decrease in the resulting cooling efficiency of the impacting drop is yet not quantitatively understood, here we experimentally study the impact of such water drops on smooth heated surfaces of various substances. We demonstrate that, in contrast with previous results for other liquids, water exhibits spray in vertical direction when impacting sapphire and silicon. We show that this typical spray formation during impact is a result of the local cooling of the plate. This is surprising since these two materials were considered to remain isothermal during impact. As a result, we conclude that the thermal time scale of the system is not solely determined by the solid thermal properties, but also by those of the liquid.

\footnotetext{
${ }^{\dagger}$ Michiel A. J. van Limbeek* , Paul B.J. Hoefnagels* , Chao Sun and Detlef Lohse, submitted for publication * Both authors contributed equally on this publication
} 


\subsection{Introduction}

Many spray-cooling applications potentially risk the so-called burn-out phenomenon: at a certain temperature the cooling efficiency drops significantly, potentially damaging expensive equipments or products. In this situation, direct contact between the solid and liquid is prevented by an insulating vapour layer, originating from the evaporating drop and separating it from the solid. The temperature at which this occurs is called the Leidenfrost temperature $T_{\mathrm{L}}[4,7,15]$ and depends on thermo-physical properties of both the solid and the liquid, surface roughness and the impact velocity of the drop $U[45,50,67]$. For obvious reasons, the prediction of $T_{\mathrm{L}}$ and the stability of the vapour film is of great importance.

For large scale cooling applications, water is used frequently since it is omnipresent, inexpensive and has a large heat capacity. Therefore, it might not be a surprise that the phenomenon was first reported for water. By varying pressure, impact velocity, plate material, etc. $[8,10,36,81,95,96]$, many studies were focussed on the prediction of $T_{\mathrm{L}}$. Most studies however make use of side-view observations, which is unable to image the liquid-solid interface, while it is at this interface, where heat is transferred between the drop and the plate.

The present study aims to unite the existing literature by studying changes of the solidliquid interface during the impact process for different velocities and plate temperatures, using the recent development of frustrated total internal reflection imaging (FTIR). This technique enables us to clearly discriminate between wetted areas with both high spatial and temporal resolution. By identifying the various boiling regimes we shed new light on the gradual change from contact boiling to Leidenfrost boiling. We will present evidence of cooling effects on a sapphire plate, in contrast with previous claims of sapphire being isothermal.

\subsection{Methods}

Water drops were dispensed from a fine needle by a syringe pump from a height between $0.5 \mathrm{~cm}$ and $40 \mathrm{~cm}$. Gravity and surface tension controlled the drop size $D_{0}$ at pinch off to be $3.8 \mathrm{~mm}$ and accelerated the drop to impact the plate with velocities $U$ ranging from $0.3 \mathrm{~m} / \mathrm{s}$ to $2.7 \mathrm{~m} / \mathrm{s}$. In most experiments the plate was a sapphire right-angle prism (with side surfaces of $\left.(25 \times 25) \mathrm{mm}^{2}\right)$ of refractive index $n=1.76$ placed in a aluminium heating block. The plate was kept at a constant temperature $T_{\mathrm{s}}$ by an electrical heater with a PID-control unit. For additional experiments a silicon wafer of thickness $0.5 \mathrm{~mm}$ and a silicon plate of thickness $5 \mathrm{~mm}$ (ThorLabs WG81050 silicon window) was used for comparison with existing literature. Prior to impact, the top surface temperatures were measured by a surface probe. All impacts were recorded by a side-view camera (Photron Fascam APX) at $5000 \mathrm{fps}$, while the transparency of the sapphire also enabled the study of the liquid-solid interface using bottom view. The technique used is based on frustrated total internal reflection (FTIR) imaging, where monochromatic parallel light (wavelength: $643 \mathrm{~nm}$ ) is reflected internally on the rectangular phase of the prism, see Fig. 6.1 and chapter 2 . The angle of incidence is chosen such that it exceeds the critical angle $\phi>\tan (1 / n)^{-1}$. Wherever a drop touches the prism, the refractive index changes locally, enabling light to propagate into the drop, resulting in a black 


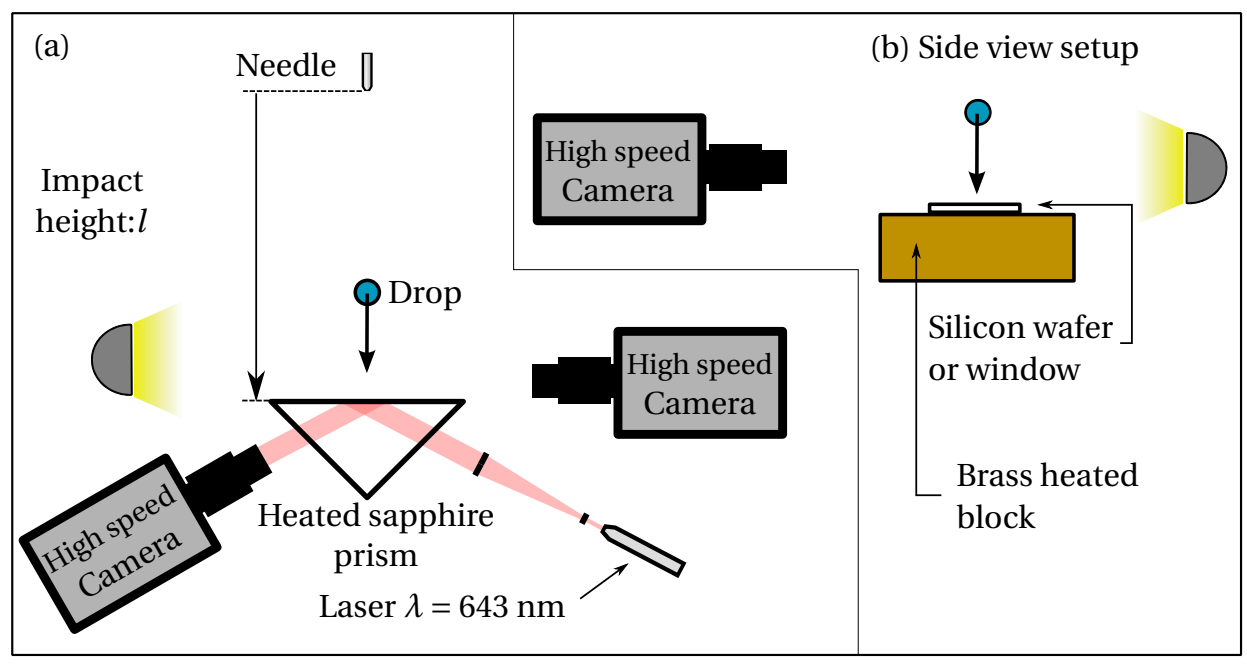

Figure 6.1: Schematic of the two experimental setups used, depending on the plate material. In addition to the side view observation we employ in the case of the transparent sapphire prism (a) a measurement technique based on frustrated total internal reflection (FTIR) to study the liquid-solid interface. Since silicon is non-transparent for light, only a side view is possible (b).

area on the camera $[45,53]$, allowing for a detailed study of dynamics present at the liquid-solid and liquid-vapour interface. To record the FTIR images, we used a high speed camera (Photron Fastcam SA1.1) at 10000 fps, equipped with a long-distance microscope (Navitar 12X Telecentric zoom system). Although both the prism and silicon surfaces have optically smooth, their thermal conductivity $k_{\mathrm{s}}$ differ significantly: 32 to $153 \mathrm{~W} /(\mathrm{mK})$ respectively, though the product of the densities and specific heats are of the same order: 3080 to $1634 \mathrm{~kJ} /\left(\mathrm{Km}^{3}\right)$.

\subsection{Results and Discussion}

A comparison of water drops impacting smooth silicon surfaces of different thicknesses can be seen in Fig. 6.2. Based on the criteria by Tran et al. [8], such spray shown in Fig. 6.2b indicates that the dynamic Leidenfrost temperature $T_{\mathrm{L}}$ has yet not been reached. The absence of the water spray in the case of a thick silicon window, shown in Fig. 6.2a, indicates that the finite plate thickness plays an important role for $T_{\mathrm{L}}$ here. Indeed, when estimating the thermal boundary layer $\delta_{\text {th }}$ developing inside the material, one finds it to be approximately $\sqrt{\frac{k_{\mathrm{s}}}{\rho C p_{\mathrm{s}}} \frac{D_{0}}{U}} \approx 0.5 \mathrm{~mm}$, where we use the impact time scale $\tau=D_{0} / U$ as the typical exposure time for the surface to the cooling of the drop. Since this is of the same order as the thickness of the silicon wafer, we propose that during the impact, the cooling front penetrates through the thin wafer and as a consequence, the heat transport towards the top surface is reduced. Hence, to maintain a drop levitated, a higher $T_{\mathrm{L}}$ is expected for such plates. In the thick silicon plate on the 
other hand, being $5 \mathrm{~mm}$ thick, heat can still be transported from the bulk of the plate, keeping the drop levitated during the full exposure time, see the sketches (c) and (d), resulting in a lower $T_{\mathrm{L}}$.

The spray observed is often related to the bursting of bubbles [66,97], see the sketch of Fig. 6.3. Two mechanisms are here considered to be of importance: the top surface of the drop should reach the bubble and therefore, a lower bound on the impact velocity can be expected. Secondly, the bubble should be a closed pocket, not connected to the surroundings.

The presence of a spray during the impact of drops was also found when impacting ethanol drops on a glass plate. A direct comparison between impacts on glass and sapphire of similar smoothness $(<10 \mathrm{~nm})$ is presented in Fig. 6.4. It was found $[45,57]$ that the difference in thermal conductivity caused the glass plate to cool down during the impact. Dendritic fingering structures of wetted areas with entrapped bubbles inside were observed in the case of glass $[56,57]$ by frustrated total internal reflection (FTIR) imaging. Since spray was only found in the case of cooling of the plate, it strengthens the suggestion that the spray in the present study in Fig. 6.2 is therefore also related to cooling of the solid. Cooling effects can therefore be observed by either a finite thickness of the plate or the plate lacking the ability to transport heat fast enough, originating from its low thermal diffusivity.

To further understand how the cooling of the plate affects the dynamic Leidenfrost temperature of water we employ a sapphire prism which, due to its transparency to light, enables us to study the wetting of the plate by the use of FTIR. This way we can investigate the development of the solid-liquid interface and investigate its dynamics over time.

\subsubsection{Phase Diagram}

Using our FTIR measurements we can discriminate the spreading radius $R_{\mathrm{S}}$ from the wetting radius $R_{\mathrm{W}}$ (see the sketches in Fig. 6.5) and study their behaviours over time. Based on this, three main regimes can be identified for the impact dynamics [45]: at low surface temperature the drop is in direct contact with the surface, i.e. $R_{\mathrm{s}}=R_{\mathrm{w}}$, referred to as the contact boiling regime. At high temperature no contact is observed, hence $R_{\mathrm{W}}=0$, called the Leidenfrost boiling regime. In between the transition boiling regime exists where partial touchdown is observed and $R_{\mathrm{S}}>R_{\mathrm{W}}$. Based on these classifications, we obtain the phase diagram shown Fig. 6.5, together with the schematics of the different regimes. The phase diagram shows the observed boiling behaviour when varying the initial plate temperature $T_{\mathrm{s}}$ and impact velocity $U$. The shaded area represents conditions where a spray was observed by the side-view camera. With increasing temperature of the plate, more vapour is generated, until the Leidenfrost regime is reached. Since here no touch-down is observed, no bubbles are formed to pierce the flattening drop and release a spray by bursting. Re-examination of the dataset from van Limbeek et al. [57] for ethanol drops impacting on glass (thickness of $1 \mathrm{~mm}$ ) revealed the presence of a spray for all impacts in the transition boiling regime as well, though it was not observed for ethanol impacts on sapphire. 
(a) Thick silicon plate $T_{\mathrm{s}}=365^{\circ} \mathrm{C}$

$0 \mathrm{~ms}$

$1 \mathrm{~ms}$

$3 \mathrm{~ms}$

$5 \mathrm{~ms}$

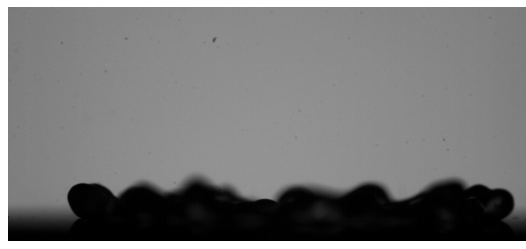

(c)

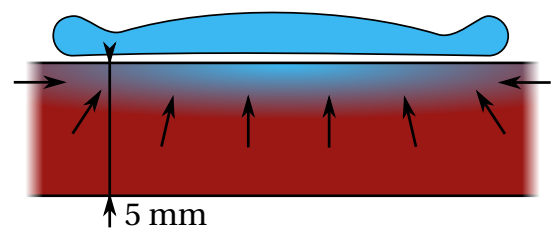

(b) Thin silicon wafer $T_{\mathrm{s}}=365^{\circ} \mathrm{C}$
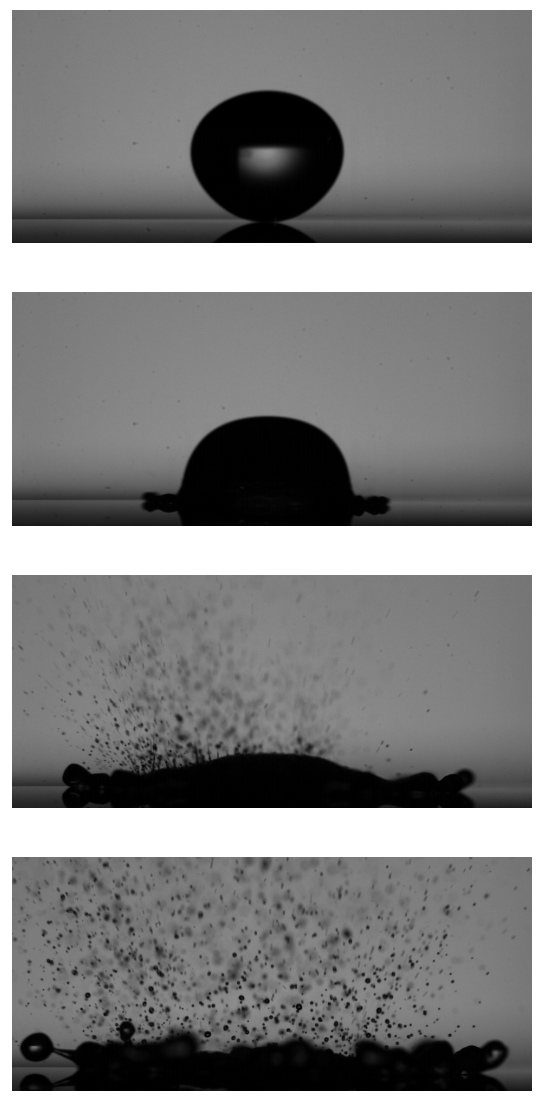

(d)

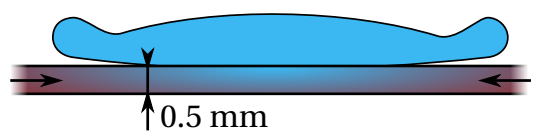

Figure 6.2: Series of images of water drops impacting a silicon window of thickness $5 \mathrm{~mm}$ (a) and a $0.5 \mathrm{~mm}$ thick silicon wafer (b), initially at $T=365^{\circ} \mathrm{C}$. The striking difference is the ejection of a droplet spray for the latter one, indicating an elevation in the Leidenfrost temperature for this system. In the sketches (c) and (d) the development of the thermal boundary layer is indicated in blue, where the arrows show the dominant heat fluxes. For a thin plate, only heat can be provided from the periphery of the impact area, while a thick window is able to also provide heat from below. 
(a)

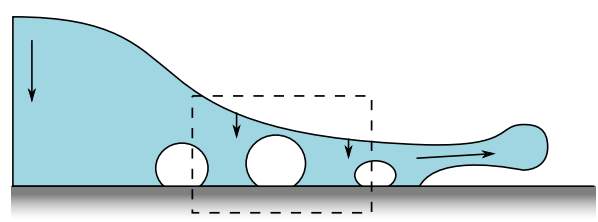

(b)

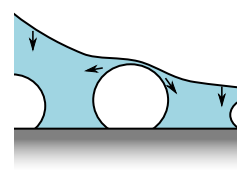

(c)
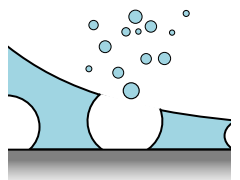

(d)

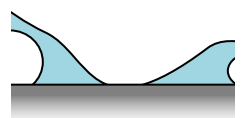

Figure 6.3: Schematic of bubble bursting mechanism. While the drop spreads as a result of the impact, the top of the drop approaches the with bubbles covered wall (a). The drainage of the thin film at the top of the bubble (b) causes the bubble to burst, emitting a spray (c). Further fragmentation of the drop can start from this location (d).

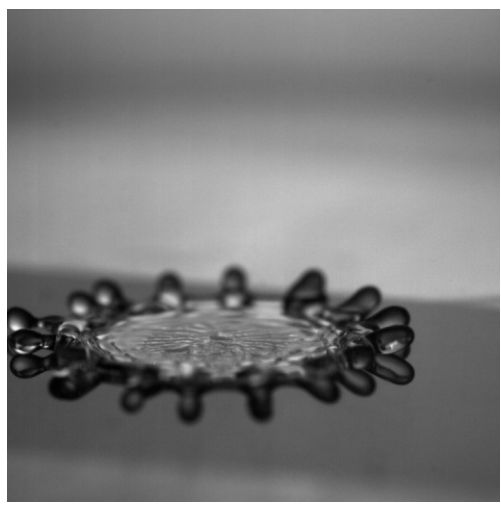

(a)

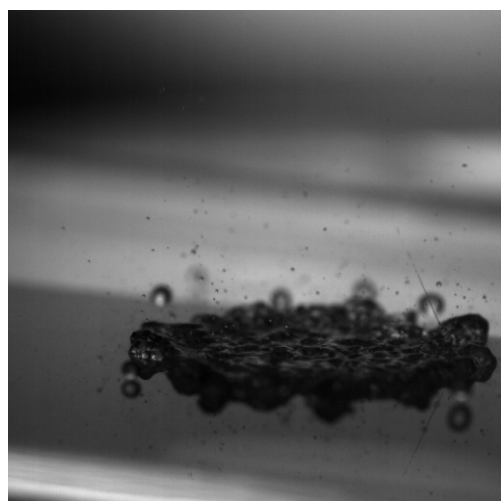

(b)

Figure 6.4: Snapshots of ethanol drops impacting on sapphire (a) and a glass plate (b), initially at a temperature of $200^{\circ} \mathrm{C}$ and $207^{\circ} \mathrm{C}$, respectively. The presence of spray in the latter correlates with the cooling of the plate. Note that this spray disturbs the top surface: the ethanol surface is much more smooth. 


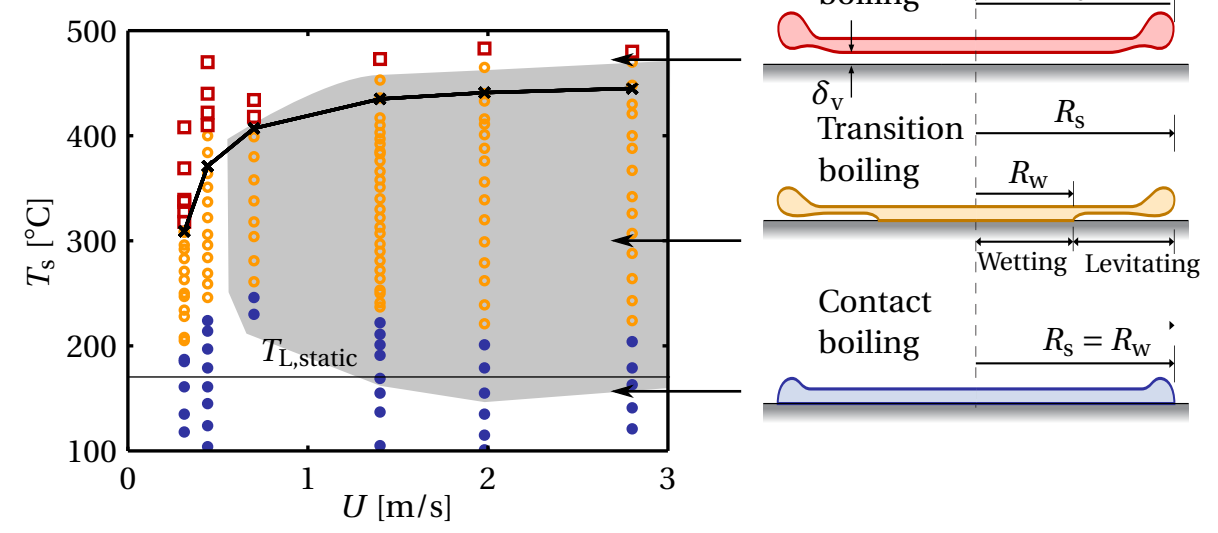

Figure 6.5: Phase diagram of the observed boiling behaviours for water drops impacting a heated sapphire prism. Three regimes can be identified, based on the difference in wetting and spreading radius, see the sketches on the right. The shaded area denotes the conditions for which a vertical spray was observed.

\subsubsection{Cooling effect}

A more in-depth analysis of the FTIR data reveals a variety of phenomenon in the transition boiling regime, as shown in Fig. 6.6. Here three different stages of the evaporative process are shown: over time, the drop is first levitated above the plate, similar to the Leidenfrost case. This situation however cannot be sustained and the drop begins to touch the plate at random locations for a short period of time, which is referred to as unstable boiling. After a certain time after the drop impact, the cool-down time $t_{\mathrm{cd}}$, some of these locations remain wetted for a long time as the plate is cooled down below the static Leidenfrost temperature [57], referred to as stable boiling.

Whether or not all three phases are observed and at which time after the impact depends strongly on the plate temperature and impact velocity $U$ of the drop. For low plate temperatures, only stable boiling is observed, previously identified as contact boiling + rim hoovering [57]. The highest temperatures for which contact still is observed only exhibits a change from Leidenfrost boiling into unstable boiling: due to the finite residence time of the drop near the plate, the plate cannot cool down enough to reach stable boiling. Since no cooling time can be observed, these measurements are not included in Fig. 6.8 and subsection 6.3.3.

In between, the boiling changes from unstable to stable boiling or even through all three phases: from an initially Leidenfrost state via unstable boiling into stable boiling. One can expect the lowest plate temperature at which initially the drop remains separated from the plate (levitating) to be close to the dynamic Leidenfrost temperature $T_{\mathrm{L}}$ for an perfect thermal conducting solid. All states, but the contact boiling + rim hoovering state, are a result of the finite heat transfer rate through the solid towards the surface of the prism, referred to as vapour cooling [57]. The downward momentum forces the drop within a few hundred nanometer from the plate and the resulting heat 
$\mathrm{t}=0.8 \mathrm{~ms}$

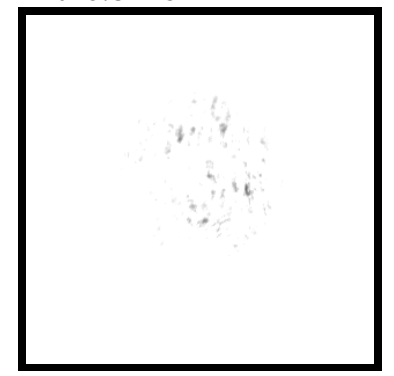

$\mathrm{t}=2.6 \mathrm{~ms}$

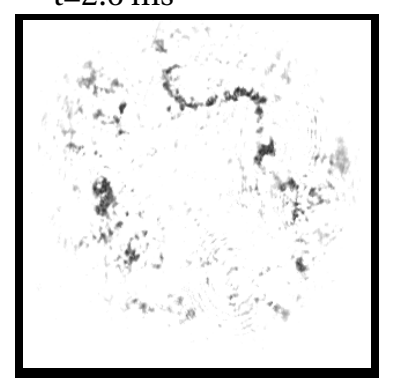

$\mathrm{t}=5.0 \mathrm{~ms}$

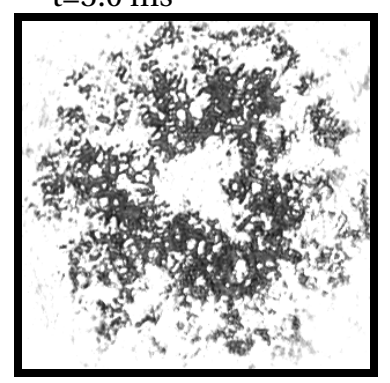

$\mathrm{t}=0.9 \mathrm{~ms}$

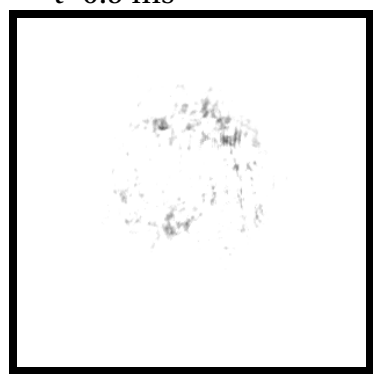

$\mathrm{t}=2.7 \mathrm{~ms}$

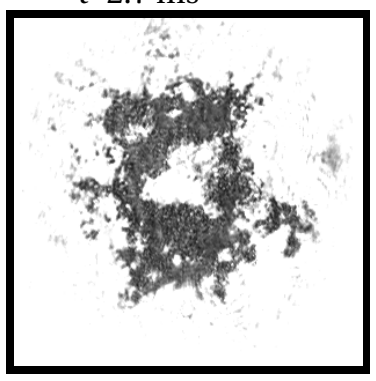

$\mathrm{t}=5.1 \mathrm{~ms}$

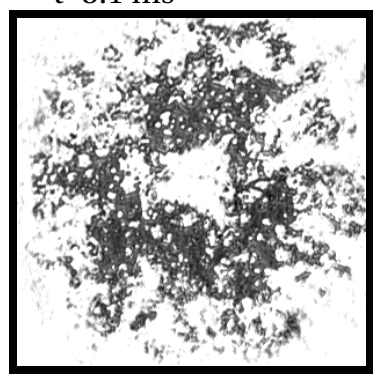

$\mathrm{t}=1.0 \mathrm{~ms}$

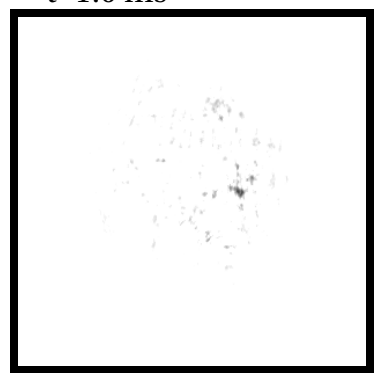

$\mathrm{t}=2.8 \mathrm{~ms}$

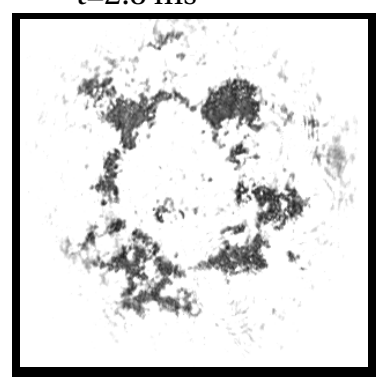

$\mathrm{t}=5.2 \mathrm{~ms}$

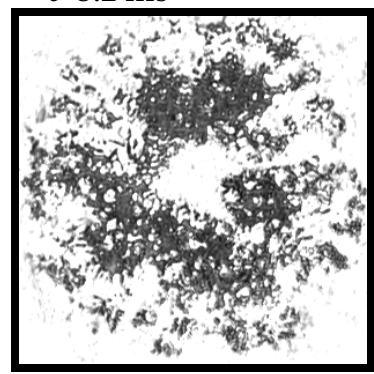

Figure 6.6: Recordings of a water drop impacting a sapphire plate initially at $T_{\mathrm{s}}=350^{\circ} \mathrm{C}$ with a velocity $U=0.4 \mathrm{~m} / \mathrm{s}$. Three stages can be identified: first the drop is (temporary) in Leidenfrost state (top row), as the drop is only visible in gray-scale (see chapter 2). This stage is succeeded by unstable boiling (middle row) where the drop touches the plate for a very short period. In the final stage, the drop is in stable boiling (bottom row) as the wetting pattern show little change in time. The indicated time is the time after impact, as identified by the side-view camera. The displayed measurement corresponds to the point in the parameter space which is marked with a star. 


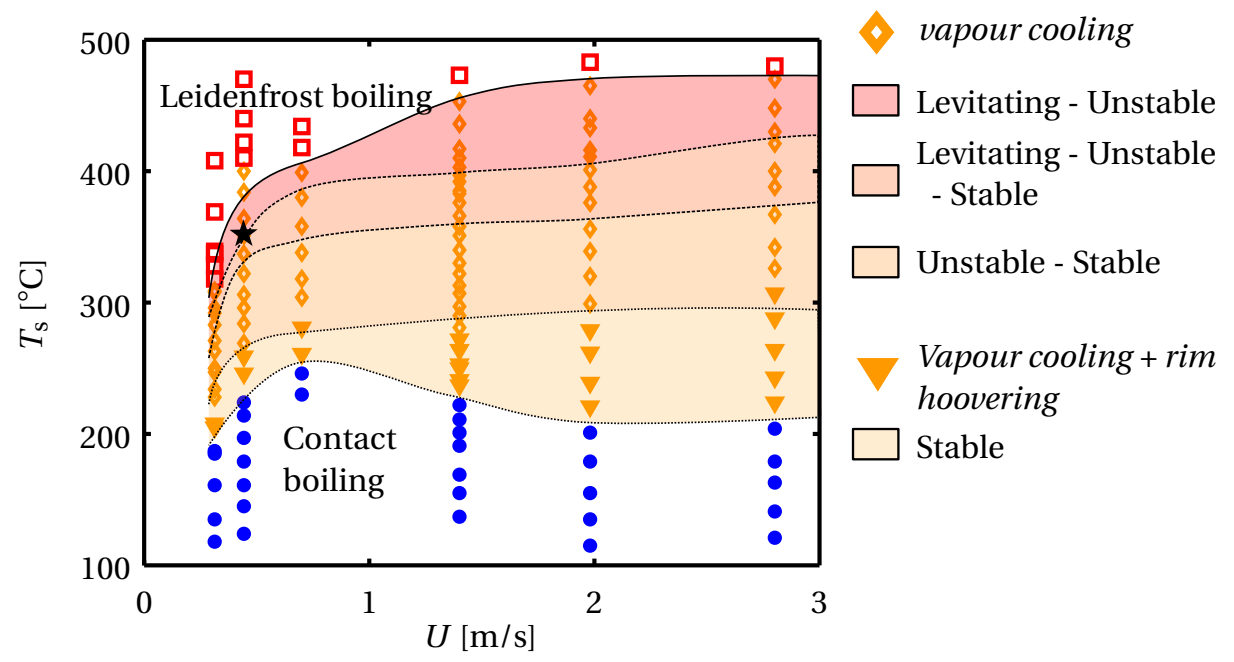

Figure 6.7: Detailed phase diagram displaying the gradual change in boiling behaviour from contact to Leidenfrost boiling. The FTIR-images of Fig. 6.6 correspond to the point in the parameter space which is marked with a star.

flux consumed by the evaporation of the drop is large enough to locally cool the prism significantly.

Figure 6.7 shows the inclusion of the new subregimes into the former phase diagram of Fig. 6.5, where the conditions exhibiting only stable boiling are represented by inverted triangles. Our observations however are in contrast to previous works using ethanol and FC-84 $\left(C_{7} F_{16}\right)$ drops, where sapphire was shown by FTIR to remain isothermal during the impact $[45,57]$. A possible explanation might be the difference in enthalpy of vaporization, but from the current work it is yet unclear to make a conclusive explanation.

\subsubsection{Thermal timescale}

The cool down time $t_{\mathrm{cd}}$ can be measured using the correlation between two successive frames, see Fig. 6.8. In this example, the drop is initially only visible in gray values as the drop is not in direct contact with the plate and hence the correlation coefficient is almost zero. After $1.4 \mathrm{~ms}$ the drop start to touch the plate at random locations, but their rapid disappearance over time result still in a low coefficient. As a result of the cooling of the plate, from $3 \mathrm{~ms}$ the wetted areas tend to stabilize, the correlation between successive images increases, resulting in stable boiling at $4.2 \mathrm{~ms}$, which is identified as the cool down time $t_{\mathrm{cd}}$.

Using this method, we obtain $t_{\mathrm{cd}}$ for various initial plate temperatures and impact velocities, presented in Fig. 6.8(b). Two trends are clearly visible: first the cool-down time increases with increasing plate temperature, in agreement with previous results [57]. However, $t_{\text {cd }}$ is found to strongly depend on $U$, decreasing with increasing impact 

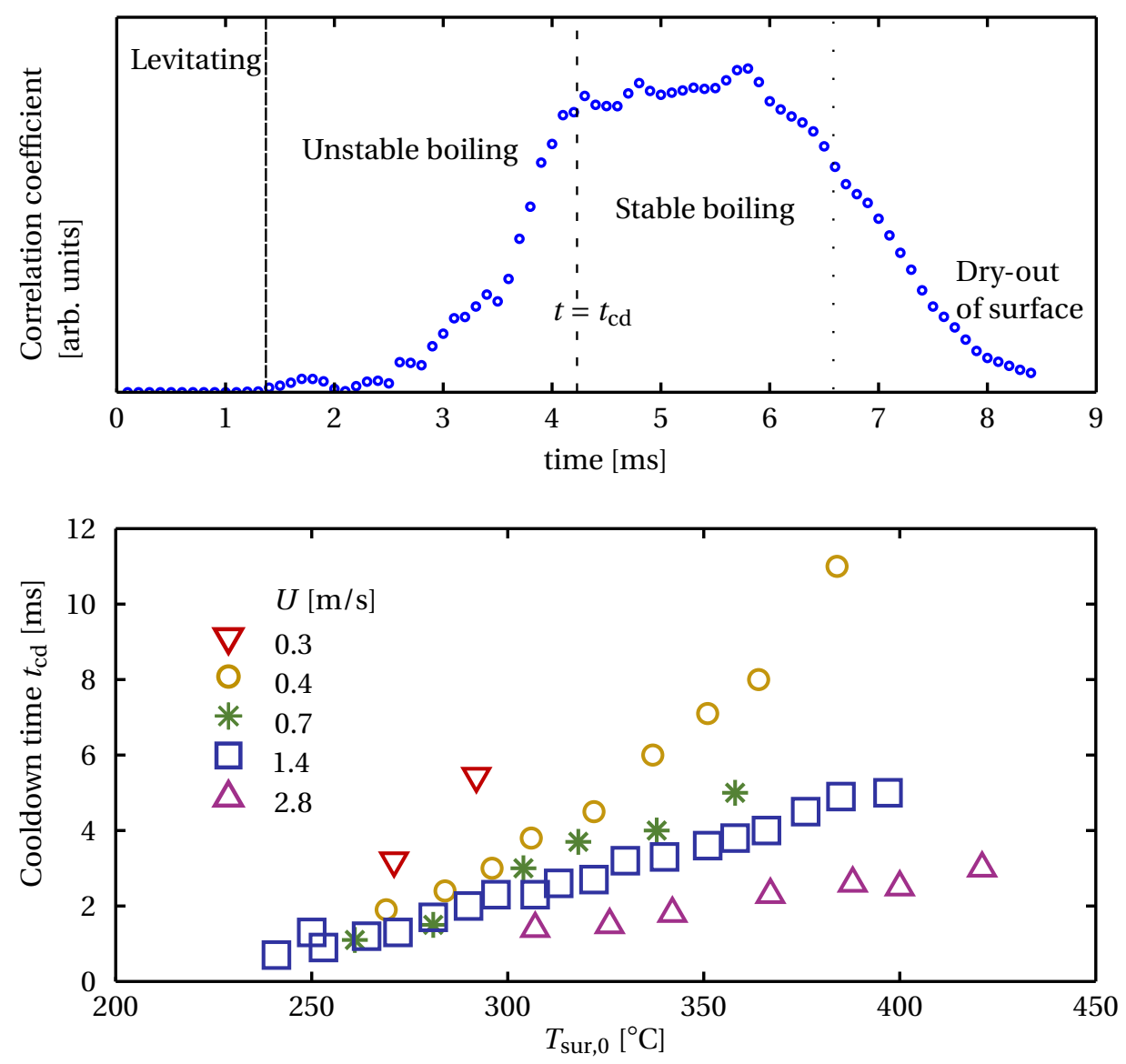

Figure 6.8: Evolution of the correlation coefficient between two successive frames (a), corresponding to the measurement shown in Fig. 6.6. A low correlation is found while the drop is still hovering above the plate during the (temporary) Leidenfrost boiling. Once (brief) contact is established (dashed line), the drop is in the unstable boiling state. The coefficient increases only slightly, while a sharp increase can be found when wetted patches remain for a long period on the surface (stable boiling). We define the cool-down time $t_{\mathrm{cd}}$ at the moment when the coefficient reaches its saturation (dashdotted line). Eventually the drop boils away from the plate, starting from the dashed line. In (b) we show the cool-down time $t_{\mathrm{cd}}$ for different impact velocities and initial plate temperatures. 
velocity.

To quantify the cooling of the plate using our measurements of $t_{\mathrm{cd}}$, we adopted the analysis method from van Limbeek et al. [57] to obtain the thermal time scale $\tau_{\text {th }}$ for the cooling in the plate. Since we have seen that $t_{\mathrm{cd}}$ depends on $U$, we expect $\tau_{\text {th }}(U)$ and hence the analysis will be performed for every $U$ separately . The separation of length scales between the vapour thickness and the drop radius justifies the use of a one-dimensional conduction model $\partial_{t} T=\frac{k_{\mathrm{s}}}{\rho_{\mathrm{s}} C p_{\mathrm{s}}} \partial_{x x} T$, where the plate of thermal conductivity $k_{\mathrm{s}}$, density $\rho_{\mathrm{s}}$ and specific heat $C p_{\mathrm{s}}$ is initially at $T=T_{\mathrm{s}}$. The top surface of the plate is subject to the constant flux boundary condition $\left.k_{\mathrm{s}} \partial_{x}\right|_{x=0}=\bar{h}\left(T_{\text {sur }}-T_{\text {sat }}\right)$, where $\bar{h}$ is the time-averaged heat transfer coefficient and $T_{\text {sur }} \equiv T(x=0)$. The analytic solution to the problem is well known $[1,10,72,92]$ and yields for the cooling of the top surface:

$$
\frac{T_{\text {sur }}(t)-T_{\text {sat }}}{T_{\text {sur }, 0}-T_{\text {sat }}}=\exp \left(\frac{t}{\tau_{\text {th }}}\right) \operatorname{erfc}\left(\sqrt{\frac{t}{\tau_{\text {th }}}}\right),
$$

where $\tau_{\text {th }}=k \rho_{\mathrm{s}} C p_{\mathrm{s}} \bar{h}^{-2}$. We have seen that at $t=t_{\mathrm{cd}}$, the plate has cooled down to the static Leidenfrost temperature [57], $T_{\mathrm{sur}}\left(t=t_{\mathrm{cd}}\right)=170^{\circ} \mathrm{C}$, which we measured separately. This enables Equation 6.1 to be solved for the left unknown: the thermal time scale $\tau_{\text {th }}$ :

$$
\frac{T_{\text {sur }}\left(t_{\mathrm{cd}}\right)-T_{\text {sat }}}{T_{\text {sur }, 0}-T_{\text {sat }}}=\frac{170^{\circ} \mathrm{C}-100^{\circ} \mathrm{C}}{T_{\text {sur }, 0}-100^{\circ} \mathrm{C}}=\exp \left(\frac{t_{\mathrm{cd}}}{\tau_{\text {th }}}\right) \operatorname{erfc}\left(\sqrt{\frac{t_{\mathrm{cd}}}{\tau_{\text {th }}}}\right),
$$

By using measurements of $t_{\text {cd }}$ for impacts at various initial plate temperature we establish a fit as shown in Fig. 6.9a. Here, every symbol represent the measured $t_{\mathrm{cd}}$, depending $T_{\text {sur, } 0}$ and grouped by impact velocity. The curves shows Equation 6.2, using the thermal time scale $\tau_{\text {th }}$ as fitting parameter. The impact velocity dependence of $\tau_{\text {th }}$ can be observed when plotting $\tau_{\text {th }}(U)$ (Fig. 6.9b), inversely scaling with $U$ (inset). The velocity dependency of $\tau_{\text {th }}$ arises from the fact that, for higher impact velocities, the drop is forced more strongly onto the plate for early times [30,35,39]. Since $\bar{h} \approx \frac{1}{t} \int_{0}^{t} k_{\mathrm{v}} \frac{\Delta T(\tilde{t})}{\delta(\tilde{t})} \mathrm{d} \tilde{t}$, a higher initial forcing reduces $\delta$ and hence decreases $\tau_{\text {th }}$.

\section{Exposure time to cooling: van Limbeek number}

To quantify the significance of cooling, it is interesting to compare $\tau_{\text {th }}$ with typical exposure time of phenomenon. In the present case of an impacting drop, we therefore use the residence time of the drop near the plate, for which we take the impact time scale $\tau=D_{0} / U$. For this ratio we can define the following dimensionless number:

$$
L i=\frac{\tau}{\tau_{\text {th }}} .
$$

Two extremes can be addressed easily: for $L i \ll 1$, the plate remains isothermal during all stages of the impact process. This is usually the case for good thermal conducting materials. Shortly after the drop touch-down due to the high pressure zones arising from the impact, the wetted areas are boiled away and the drop becomes levitated for the remainder of the impact time. For $L i \gg 1$ however, the limitation in heat transfer lowers 

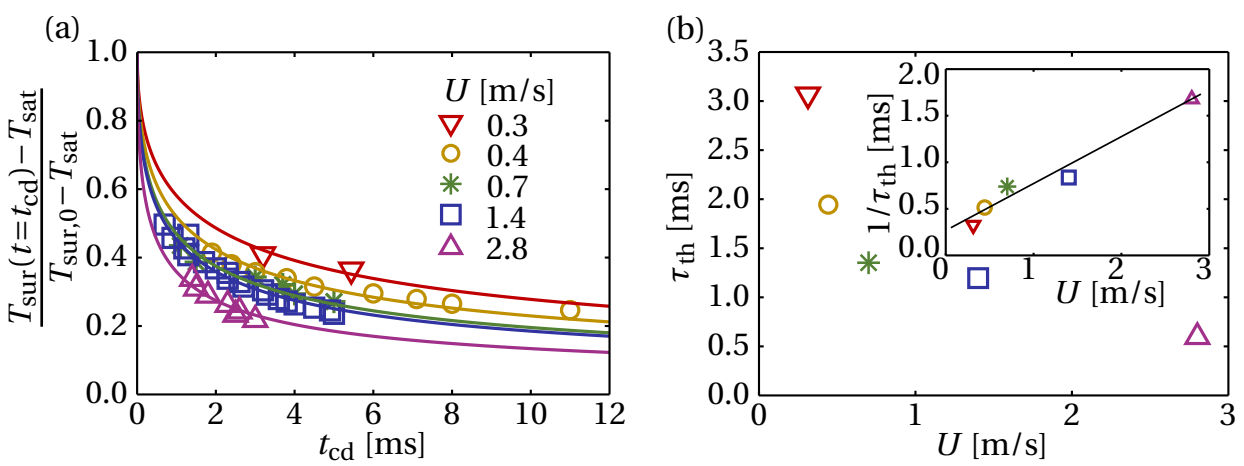

Figure 6.9: Over time, the (nondimensionalized) plate temperature decreases with time. At $t=t_{\mathrm{cd}}$, the plate has cooled down to the static Leidenfrost temperature: $T_{\text {sur }}\left(t=t_{\mathrm{cd}}\right)=$ $170^{\circ} \mathrm{C}$. The data points are plotted in the left panel for various initial plate temperature $T_{\text {sur, } 0}$ and impact velocities $U$ representing separate experiments. By fitting Equation 6.1 (see main text). the thermal time scale $\tau_{\text {th }}$ is obtained and presented in (b) as a function of the impact velocity. The inset reveals $\tau_{\text {th }}^{-1} \propto U$.

the surface temperature below the static Leidenfrost temperature [57] significantly. Even drops which remain levitated in the initial stages can touch-down eventually due to the insufficient heat transfer towards the drop interface. This is the case of solids of poor thermal conductivity or for plate of finite thickness as we have seen in the case of a water drop impacting on a silicon wafer (Fig. 6.2b).

In the present case of water impacting on sapphire we obtained for all impact velocities $L i$ to be between 1.7 and 3, and the $\tau_{\text {th }}$ has become a function of $U$ itself. Since both time scales are comparable, it is not surprising that we observed a smooth transition from the contact boiling regime into the Leidenfrost regime for increasing initial plate temperature. It is expected that all stages are present for all situations where $L i \gg 1$, but the shorter $\tau_{\text {th }}$, the faster the various stages succeed one another. In those cases, to still observe them, the frame rate of the camera must allow for a good temporal resolution.

\subsubsection{Reflection on existing literature}

If we compare our measurements with the results by Tran et al. [8], we obtain good agreement for the dynamic Leidenfrost temperature of water, see Fig. 6.5. We found that the occurrence of spray from side-view observation strongly correlates with the presence of wetted areas with entrapped bubbles on the solid surface, as observed by FTIR. Since the surface to cools down as a result of the presence of the drop, the wetted areas with their entrapped bubbles can survive on the surface, resulting in the spray formation by the bursting of the bubbles at the top surface of the drop, in agreement with previous studies on spray formation $[66,97]$. Furthermore, we find the boundary between contact boiling and transition boiling to be weakly dependent on the impact velocity, similarly as reported for ethanol drops impacting on glass [57] and on sapphire [45,69]. Since no cooling was found in the latter two studies, it is not surprising that no spray is formed: 
the wetted area in the transition regime is boiled away long before the top interface of the drop has come close to the bubbles. Because of the absence of the spray, Staat et al. [69] identified all impacts above this boundary to be film boiling, though in later studies the lower part of this regime was named and identified as the transition boiling regime $[57,57]$.

\subsection{Conclusion}

Our study of water drops impacting on smooth surfaces has revealed that the presence of a spray is a signature of cooling effects in the plate. We have shown that water drops cool down the plate at a rate which depends on the impact velocity. Utilizing side view imaging we found that the dynamic Leidenfrost temperature was found only when the plate is subject to cooling as on a good thermal conductor, the wetted area from the impact boils away before bubbles can generate a spray by bursting at the top surface of the drop. Our study indicates that sapphire cannot always behave isothermally, as the difference in liquid properties could also play a fundamental role. This deserves more systematic studies in the future.

\section{Acknowledgements}

This work was supported by an ERC-Advanced Grant. 


\section{Chapter 7}

\section{Interferometric measurement of surface cooling by Leidenfrost drops $^{\dagger}$}

When a liquid drop is placed on a highly superheated surface, it can be levitated by its own vapour. This remarkable phenomenon is referred to as the Leidenfrost effect. The thermally insulating vapour film results in a severe reduction of the heat transfer rate compared to experiments at lower surface temperatures, where the drop is in direct contact with the solid surface. A commonly made assumption is that this solid surface is isothermal, which is at least questionable for poor thermal conducting materials, resulting in an over-estimation of the surface temperature and heat transfer for such systems. Here we aim to obtain more quantitative insight in how surface cooling affects the Leidenfrost effect. We develop a technique based on Mach-Zehnder interferometry to investigate the surface cooling of a quartz plate by a Leidenfrost drop. The threedimensional plate temperature field is reconstructed from interferometric data by a novel Abel inversion method. By this method we are able to quantitatively measure the local cooling inside the plate, which can be as strong as $80 \mathrm{~K}$. We develop a numerical model which shows good agreement with experiments and enables us to extend the analysis beyond the experimental parameter space. Based on the numerical and experimental results we quantify the effect of surface cooling on the Leidenfrost phenomenon. By focusing on the role of the solid surface we provide new insights on the Leidenfrost effect and demonstrate how to adjust current models to account for non-isothermal solids and recover the isothermal scaling laws for the neck thickness and evaporation rate.

\footnotetext{
${ }^{\dagger}$ Michiel A.J. van Limbeek, Martin H. Klein Schaarsberg, Benjamin Sobac, Alexey Rednikov, Chao Sun, Pierre Colinet and Detlef Lohse. Submitted for publication
} 


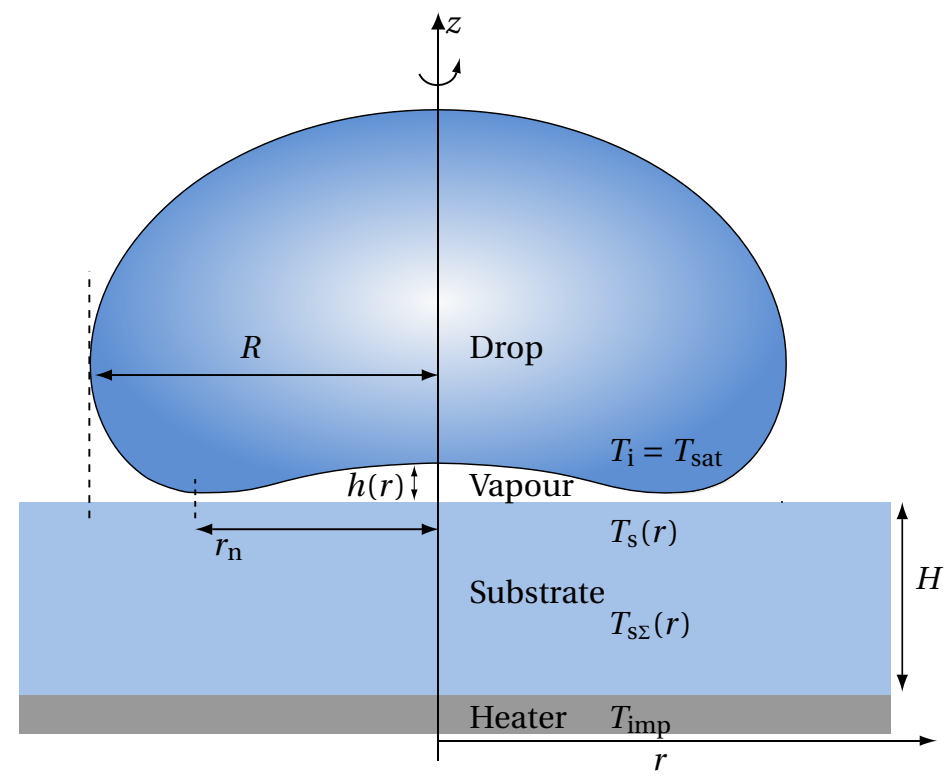

Figure 7.1: Schematic illustration of a Leidenfrost drop on a heated plate. The drop remains levitated by the vapour generated in the vapour film under the drop and escaping radially. Here $T_{\mathrm{s}}$ is the temperature in the substrate and $T_{\mathrm{s} \Sigma}$ denotes specifically the temperature distribution of the top surface. The heater maintains the imposed temperature $T_{\mathrm{imp}}$.

\subsection{Introduction}

The evaporation time of a drop brought into contact with a hot solid plate depends strongly on the solid temperature. For drops in contact with the plate (sessile drops), this time decreases with increasing plate temperature, until a sudden increase is observed. The temperature at which this increase happens is referred to as the Leidenfrost temperature $T_{\mathrm{L}}[5,7]$. The increase is caused by the formation of a vapour film under the drop. The film separates the drop from the hot plate and the generated vapour is escaping from underneath the drop (see Fig. 7.1). The viscous flow induces an overpressure, strong enough to lift the drop above the plate $[14,15,17]$. The film also limits the heat transfer towards the drop, resulting in a strongly reduced evaporation and thus an increased lifetime of the drop [4]. Elevating the plate temperature further above $T_{\mathrm{L}}$ results then again in a decrease in the lifetime of the drop. In many cooling applications, like quenching processes, superconductor cooling, heat exchangers and metal processing, the manifestation of the Leidenfrost effect is rather unfavourable as the intended cooling performance becomes inadequate. Therefore, the study of how $T_{\mathrm{L}}$ depends on both the liquid and the solid material $[10,98]$ and roughness $[18,82]$ is of great importance.

The evaporation of a Leidenfrost drop requires (latent) heat, which is taken from the plate. To estimate whether or not this influences the plate temperature, one can 
compare the residence time with the characteristic cooling time-scale of the plate [10], ranging from milliseconds to seconds. When the residence time of the drop is short (impacting drops), most solids exhibit isothermal conditions and remain at to the initial plate temperature $[8,45,69,82]$. Some plate materials however do show a signature of local cooling, despite the short exposure time to the evaporating drop [50,57,67]. However, if the residence time is of the order of seconds (sessile drops), these materials are subject to a more substantial cooling by the evaporating drop and only good thermal conductors like copper still behave isothermally [10]. When constantly feeding the drops, the system becomes time-independent, i.e. all dynamical processes reach their steady-state regime and even a good thermal conducting plate undergo a cooling of a few degrees. In the study of the Leidenfrost effect, the cooling of the plate is often neglected as little quantitative data is available. Therefore, assuming the heater setpoint to be the actual temperature under the drop results in those cases in a nonnegligible temperature over-estimation. For proper modelling of Leidenfrost drops and the interpretation of experiments, the understanding of the cooling of the plate is therefore of great importance.

Some studies focused on measuring the cooling by integrating thermocouples into the solid plate [99]. However, this technique is intrusive and limited by the finite size of the thermocouples. Furthermore, the technique only results in a local (point) measurement and does not provide the complete temperature field.

In this study we use interferometry to measure the three-dimensional temperature field in a quartz plate which is cooled by an evaporating Leidenfrost drop. The technique is an indirect method to measure temperature, concentration or pressure fields by detecting minute varies in the refractive index [100,101]. Our measurements are based on the fact that the refractive index of a solid changes with temperature, which yields a change in the optical path length. From the generated fringe patterns, obtained by a Mach-Zehnder interferometer, we can measure these changes and obtain the entire temperature field in a non-intrusive way.

To reconstruct the three-dimensional temperature field from a single interferometric projection, we develop a novel Abel inversion method, which presumes the temperature field to satisfy the steady-state heat equation (i.e. the Laplace equation) inside the plate. The complete (axisymetric) temperature field inside the plate can thereby be obtained. On the one hand, these experimental data, and in particular the measured temperature distribution at the plate surface, can directly be used in Leidenfrost drop modelling, which then amounts to the gas phase only, as for an isothermal substrate [16]. This enables us to calculate the distribution of heat (and evaporation) fluxes and vapour film thicknesses. On the other hand, these experimental data are also crucial for validating a more complete Leidenfrost model, which includes heat conduction in the solid, and which is developped and validate here. It allows us to explore the role of solid material properties beyond experimental possibilities. Based on our findings we distinguish four different limiting regimes, characterized by two non-dimensional numbers: a Biot-like number indicating whether or not the cooling of the plate is significant, and a geometric parameter describing the global shape of the fields. Finally, we give insight into how the scaling laws proposed for isothermal substrates are modified in the case of poor thermal conducting plates. 
(a)

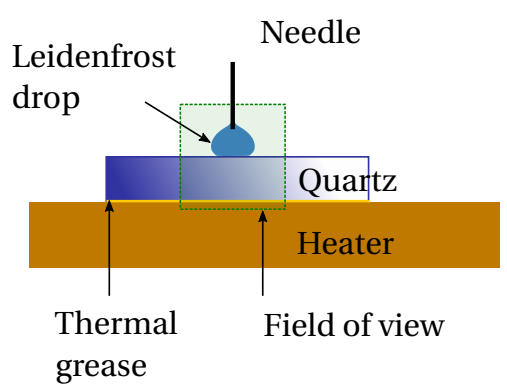

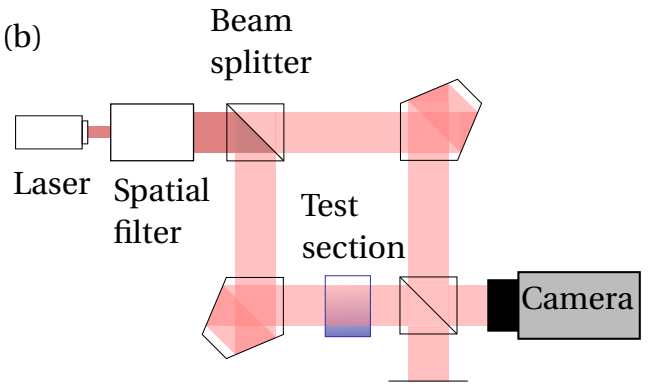

Viewing screen

Figure 7.2: Schematic illustration of the experimental setup (a) and the top view of all the optical parts of the Mach-Zehnder interferometer (b). The laser beam in (a) is orthogonal to the drawing plane and points towards the reader.

\subsection{Experimental setup and methods}

\subsubsection{Experimental setup}

Figure 7.2 shows a schematic illustration of the experimental setup. A Leidenfrost drop is obtained by placing a drop of ethanol on top of a hot solid substrate with a temperature well above the boiling point of ethanol $\left(T_{b}=79^{\circ} \mathrm{C}\right)$. The substrate is a rectangular quartz plate of dimensions $30 \times 15 \times 4.5 \mathrm{~mm}^{3}$ (length $\times$ width $W, \times$ height $H_{\mathrm{s}}$ ) placed above a brass element, which coupled with a heater, a PID controller and a thermocouple, ensures a constant imposed temperature at the bottom of the plate $T_{\mathrm{imp}}$. It is worth noting that quartz is a relatively poor thermal conductor, with a thermal conductivity $k_{\mathrm{s}}=1.4 \mathrm{~W} /(\mathrm{mK})$. The drop is fed at a constant rate from a glass capillary connected to a syringe pump. The feeding rate allows to control the drop radius $R$ (as viewed from the top). Since quartz is transparent, one can recur to a Mach-Zehnder interferometer (see the right sketch of Fig. 7.2) in order to visualize and measure the three-dimensional temperature field inside the plate, making use of the temperature dependence of its refractive index. This interferometer reveals optical path-length differences between the reference beam and the measurement beam, the latter one passing through our plate. The fringes resulting from combining the two beams are recorded using a camera. A He-Ne laser $(\lambda=633 \mathrm{~nm})$ is used as a collimated light source. The beam quality is improved by a spatial filter, which also acts as a beam expander. The interferometric setup operates in the finite-fringe width mode (see section 7.A) to reduce the influence of noise and eliminate ambiguities in post-processing. The steps to obtain the temperature field from the interferograms will be explained in the following subsection.

\subsubsection{Data processing}

Figure 7.3 shows a typical interferometry experiment with an ethanol Leidenfrost drop of size $R_{\max }=1.4 \mathrm{~mm}$ and heater temperature $T_{\mathrm{h}}=330^{\circ} \mathrm{C}$. The various figures show different steps in the data processing, to be discussed in this subsection. 


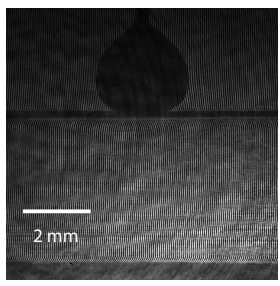

(a) $I_{\mathrm{t}}(y, z)$

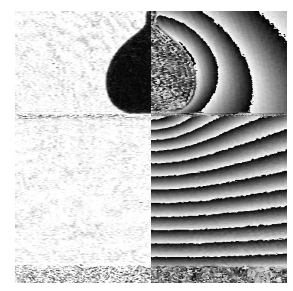

(c) $M(y, z), \psi(y, z)$

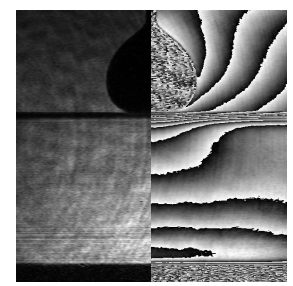

(b) $M_{\mathrm{t}}(y, z), \psi_{\mathrm{t}}(y, z)$

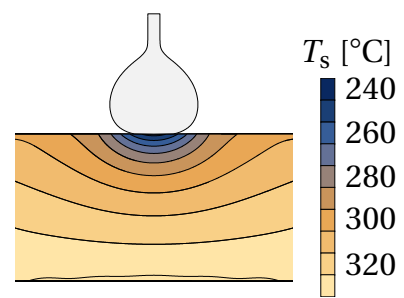

(d) $T(r, z), R(z)$

Figure 7.3: Leidenfrost interferometry experiment with an ethanol drop $\left(R_{\max } \approx \lambda_{\mathrm{c}}\right.$, $T_{\mathrm{h}}=330^{\circ} \mathrm{C}$ ), with interferogram $I_{\mathrm{t}}(\mathrm{a})$, magnitude $M_{\mathrm{t}}$ and phase $\psi_{\mathrm{t}}(\mathrm{b})$, background corrected magnitude $M$ and phase $\psi$ (c) and the reconstructed temperature field $T(r, z)$ and drop contour $R(\mathrm{~d})$. All images are on the same scale.

The interferogram $I_{\mathrm{t}}$ Fig. 7.3a is decomposed into its phase $\psi_{\mathrm{t}}$ and magnitude $M_{\mathrm{t}}$ $7.3 \mathrm{~b}$ with a Fourier transform technique [102-104]. Since all interferograms resulting from experiments are subject to some noise which distort the analysis process, a band pass filter is used to remove low frequency background intensity variation of the laser beam and high frequency noise (section 7.A). A second advantage over the infinite-fringe-width mode is its ability of detecting smaller phase variations and the unambiguous determination of the slope of phase changes.

The temperature gradient in the substrate also makes the substrate appear to be displaced slightly (on the order of $0.1 \mathrm{~mm}$ ) due to the refraction of the light. This apparent translation is determined by cross-correlating the magnitude image $M_{\mathrm{t}}$ with a magnitude image from a reference interferogram taken at room temperature. The magnitude is divided by the reference magnitude to get the magnitude image magnitude image $M$ Fig. 7.3c. The drop contour is obtained from this image with the Canny edge detection algorithm [105], from which the drop size $R_{\max }$ and the vapour film radius $R_{\mathrm{C}}$ is extracted.

The phase from the reference interferogram is subtracted to isolate the phase change $\psi$ due to the temperature change. This removes disturbances by the optical system such as inhomogeneities of the substrate width $W$ (along the direction of the laser beam), and other optical distortions.

To obtain the absolute phase difference $\phi$ we need to unwrap the wrapped phase $\psi \in[-\pi, \pi)$. We use the unwrapping algorithm from Herraez et al. [106] to unwrap the phase images, which excels in preventing the propagation of errors from local noise. 
Let $x, y$ and $z$ be the Cartesian coordinates along the length, width and height of the quartz plate, respectively. The laser beam propagates in the $x$ direction, while $(y, z)$ is the plane of the field of view. The interferometric phase $\phi(y, z)$ then depends on the refractive index $n(x, y, z)$ and the laser wavelength $\lambda$ as

$$
\phi(y, z)=\frac{2 \pi}{\lambda} \int_{-\infty}^{\infty} \Delta n(x, y, z) d x
$$

Here $\Delta n=n-n_{0}$, while $n_{0}$ is chosen to be the refractive index value at the ambient temperature $T_{0}$. Since the refractive index of fused quartz increases linearly with temperature, $\Delta n=\mathrm{d} n / \mathrm{d} T \Delta T$, we can thus relate the change in phase to the change in temperature, such that (7.1) becomes

$$
\phi(y, z)=\frac{2 \pi}{\lambda} \frac{\mathrm{d} n}{\mathrm{~d} T} \int_{-\infty}^{\infty} \Delta T_{s}(x, y, z) d y
$$

where $T_{s}(x, y, z)$ is the temperature field in the solid substrate (quartz plate), while $\Delta T_{\mathrm{s}}=T_{\mathrm{S}}-T_{0}$. A detailed description of our calibration experiment can be found in section 7.B, where we obtained $\mathrm{d} n / \mathrm{d} T=(1.20 \pm 0.01) \times 10^{-5} / \mathrm{K}$, which is in within the range of values measured for fused quartz with the method of minimum deviation at $\lambda=633 \mathrm{~nm}[94,107]$. This experiment was also used to determine how the temperature field depended on the heater temperature in the absence of a drop for different heater set points.

The largest source of deviation in our measurements is due to the heating of air around the plate, which results in an underestimation of the change in refractive index in the quartz. However, since the refractive index of air decreases with increasing temperature, with $\mathrm{d} n / \mathrm{d} T$ on the order of $-10^{-7} / \mathrm{K}$ [108], which is two orders of magnitude smaller than the value of $\mathrm{d} n / \mathrm{d} T$ for quartz, we judge this influence to be negligible.

The final step is to reconstruct the three-dimensional temperature field $\Delta T_{\mathrm{s}}(x, y, z)$ from the two-dimensional phase image $\phi(y, z)$ by Abel inversion (Fig. 7.3d) assuming the field to be essentially axisymmetric, i.e. $\Delta T_{\mathrm{s}}(r, z)$ with $r=\sqrt{x^{2}+y^{2}}$. The temperature field $\Delta T_{\mathrm{s}}(r, z)$ is then usually obtained using the inverse Abel transform of $\phi(y, z)$. However, since this procedure is sensitive to noise and exhibits diverging behaviour near the origin, it is not quite suitable to be used on our experimental data. Therefore, we propose a new inversion method based on the basis function expansion method [109] for expressing the experimentally obtained phase field $\phi(y, z)$ as a series expansion in terms of axisymmetric modes of $\Delta T_{\mathrm{S}}(r, z)$. The two expansions will obviously be related by means of Equation 7.2. $\Delta T_{\mathrm{s}}(r, z)$ is assumed to be axisymmetric and governed by the steady-state heat (Laplace) equation $\nabla^{2}\left(\Delta T_{\mathrm{s}}\right)=0$. Thus, a set of Fourier-Bessel functions can be used for $\Delta T_{\mathrm{s}}(r, z)$, see section 7.C, which when integrated according to Equation 7.2 yields the corresponding set of functions for $\phi(y, z)$. The coefficients of the series expansions for $\Delta T_{\mathrm{s}}(r, z)$ and $\phi(y, z)$ herewith obviously coincide. They are determined by fitting the experimental data for $\phi(y, z)$. Once the coefficients are known, the temperature field $\Delta T_{\mathrm{s}}(r, z)$ gets fully determined too from its own series expansion up to a constant. We fix this constant such that the average temperature on the bottom of the substrate is equal to the heater set point temperature $T_{\mathrm{imp}}$. 


\subsection{Formulation of the theoretical model}

In order to model the experimental situation, we consider an axisymmetric Leidenfrost drop levitating over a hot solid substrate. The present model is aimed at predicting the quasi-steady state of such a drop (the slowest process being its evaporation), including both its geometry and a possible cooling of the substrate. The drop geometry is modelled by numerically matching the hydrostatic equilibrium shape of a superhydrophobic drop (for the upper part) with the lubrication equation solution for the vapour film underlying the drop (for the bottom part), quite similarly to Sobac et al. [16]. However, unlike Sobac et al. [16], the substrate is no longer considered isothermal, and its cooling is accounted for by solving a heat transfer problem therein. As in the experiment, the substrate is assumed to consist of a horizontal plate (thickness $H_{\mathrm{s}}$ ) with the temperature kept constant at its bottom surface.

Let us first focus on the shape of the drop , following Sobac et al. [16]. The upper part of the drop, above the vapour film, is assumed to be an equilibrium shape, for which the Laplace pressure locally balances (up to a constant) the hydrostatic pressure. This simply reads

$$
\gamma \kappa+\rho_{\ell} g\left(z-z_{\text {top }}\right)=\gamma \kappa_{\text {top }}
$$

where $\rho_{\ell}$ is the liquid density, $\gamma$ is the surface tension, and $g$ is the gravitational acceleration. Here, $\kappa$ is the local curvature of the drop surface (a function of the drop shape and its derivatives), while $\kappa_{\text {top }}$ (curvature at the top of the drop, $z=z_{\text {top }}$ ) is a free parameter controlling the drop size. For a given value of the top curvature $\kappa_{\text {top }}$, numerically integrating this differential equation from the symmetry axis yields the corresponding equilibrium shape and in particular the value of the drop radius $R=R\left(\kappa_{\mathrm{top}}\right)$. Inversely, for any given $R$, one unambiguously finds the corresponding equilibrium shape and $\kappa_{\text {top }}$. However, the shape is hereby determined just up to a vertical shift, for the value of $z_{\text {top }}$ can fully be calculated only upon the consideration of the underlying vapour film in what follows.

This "upper equilibrium drop" solution is assumed to be valid up to a (patching) point located at $r=R_{\mathrm{p}}$ near the exit from the vapour film, where non-equilibrium effects of evaporation and viscous pressure losses in the vapour flow are deemed to progressively come into play. In this "vapour layer region" $0<r<R_{\mathrm{p}}$, the thickness of the vapour film $h(r)$ and its slopes $h^{\prime}(r)$ are assumed to be small enough so as to use the lubrication approximation (in particular, $h \ll R$ and $\left|h^{\prime}\right| \ll 1$ ). The gas itself is assumed to be composed of pure vapour (no air) and incompressible. Then, discarding possible motions inside the drop, which is a feature of all similar models developed thus far $[14,16,17,21]$, the excess pressure (over the ambient one) in the vapour film is found from the normal stress balance at the drop surface as $P_{\mathrm{v}}=\gamma \kappa_{\text {top }}+\rho_{1} g\left(z_{\text {top }}-h\right)-\gamma \kappa$. This excess pressure drives a Stokes flow with a volumetric flux $\vec{q}_{\mathrm{v}}=-\vec{\nabla} P_{\mathrm{v}} h^{3} /\left(12 \mu_{\mathrm{v}}\right)$, where $\mu_{\mathrm{v}}$ is the vapour dynamic viscosity. Note the coefficient $1 / 12$ in the mobility factor, reflecting of no-slip conditions imposed at both the drop and solid interfaces. Assuming that heat is predominantly transferred by conduction across the film (for an $a$ posteriori estimation of the Péclet number see section 7.D), the local evaporation flux at the interface is expressed as $\mathscr{J}=L^{-1} k_{\mathrm{v}} \Delta T / h$, where $k_{\mathrm{v}}$ is the vapour thermal conductivity, $L$ is the latent heat of vaporisation, $\Delta T=T_{\mathrm{s} \Sigma}-T_{\mathrm{sat}}$ is the superheat, and $T_{\mathrm{s} \Sigma}$ is the temperature field of the substrate surface underneath the drop. Finally, the steady-state 
vapour mass conservation under the thin-film hypothesis reads $\vec{\nabla} \cdot\left(\rho_{\mathrm{v}} \vec{q}_{\mathrm{v}}\right)-\mathscr{J}=0$ (at steady state), where $\rho_{\nu}$ is the vapour density. Combining these results and assuming the axial symmetry yields the following lubrication equation for the film thickness

$$
\frac{1}{12} \frac{1}{r} \frac{\partial}{\partial r}\left(\frac{\rho_{\mathrm{v}}}{\mu_{\mathrm{v}}} h^{3} r \frac{\partial}{\partial r}\left(\rho_{\mathrm{l}} g h+\gamma \kappa\right)\right)-\frac{k_{\mathrm{v}} \Delta T}{L h}=0,
$$

where the curvature $\kappa$ is given by

$$
\kappa=\frac{\frac{\partial^{2} h}{\partial r^{2}}+\frac{1}{r}\left(1+\left(\frac{\partial h}{\partial r}\right)^{2}\right) \frac{\partial h}{\partial r}}{\left(1+\left(\frac{\partial h}{\partial r}\right)^{2}\right)^{3 / 2}},
$$

expressed in full form to patch Equation 7.4 with the top part of the drop, as expressed by Equation 7.3.

The system is characterised by large spatial temperature variations, the typical values of $\Delta T$ being generally rather comparable with the absolute temperature itself. As the liquid and gas properties are temperature-dependent, a relevant question is then at what temperature they should be evaluated in our formulation. This is more straightforward for the liquid properties: as the drop supposedly remains at (or near to) $T_{\text {sat }}$, they are just taken at $T_{\text {sat }}$. As for the vapour properties, a simplified treatment similar to Sobac et al. [16] will be used, evaluating physical properties locally at the mean temperature of the vapour film, $\left(T_{\mathrm{s} \Sigma}(r)+T_{\mathrm{sat}}\right) / 2$. Thus, the effect of a temperature variation at the top of the substrate due to the cooling enters here not only through a position-dependent superheat $\Delta T(r)$, but also through the vapour properties which vary along $r$. The vapour properties themselves are calculated as in Sobac et al [110]. For example, at a temperature of $200^{\circ} \mathrm{C}$, the values of the vapour mass density, dynamic viscosity and thermal conductivity of ethanol vapour are $\rho_{\mathrm{v}}=1.187 \mathrm{~kg} / \mathrm{m}^{3}, \mu_{\mathrm{v}}=1.436 \times 10^{-5}$ Pa.s and $\lambda_{\mathrm{v}}=0.023 \mathrm{~W} / \mathrm{m} . \mathrm{K}$, respectively.

Four boundary conditions are needed to supplement Equation 7.4 and 7.5: the symmetry conditions at $r=0$, i.e. $h^{\prime}(0)=0$ and $\kappa^{\prime}(0)=0$, while at $r=R_{\mathrm{p}}$ the solution must match with the earlier obtained upper equilibrium shape of the drop, i.e. we require the continuity of $h^{\prime}(r)$ and of $\kappa(r)$. The continuity of $h(r)$ itself here merely amounts to finding the appropriate vertical shift of the upper equilibrium shape, i.e. to determining the value of $z_{\text {top }}$.

The temperature distribution $T_{\mathrm{S} \Sigma}(r)$ at the top of the solid substrate is obtained by solving the heat conduction equation in the plate coupled with Equation 7.4. Assuming quasi-steadiness and a constant thermal conductivity $k_{\mathrm{s}}$ of the solid , the temperature field $T_{\mathrm{s}}(r, z)$ in the solid is governed by the axisymmetric Laplace equation

$$
\frac{1}{r} \frac{\partial}{\partial r}\left(r \frac{\partial T_{\mathrm{s}}}{\partial r}\right)+\frac{\partial^{2} T_{\mathrm{s}}}{\partial z^{2}}=0
$$

The following boundary conditions close the problem finally: the symmetry condition $\partial_{r} T_{\mathrm{s}}=0$ at $r=0$, an imposed temperature at the substrate bottom, i.e. $T_{\mathrm{s}}=T_{\mathrm{imp}}$ at $z=$ $-H_{\mathrm{s}}$, and heat fluxes continuity at $r=R_{\mathrm{s}}$ and $z=0$. At the plate sides, an experimental 
evaluation of heat losses to the air due to convection proved them to be negligible. Consequently, an insulating condition will be imposed thereat, i.e. $\partial_{\mathrm{r}} T_{\mathrm{s}}=0$ at $r=R_{\mathrm{s}}$. For mathematical simplicity, the plate is assumed to be cylindrical with radius $R_{\mathrm{s}}$ in the model, while it is rectangular in the experiment. $R_{\mathrm{S}}$ is taken as half the plate width, i.e. $R_{\mathrm{S}}=7.5 \mathrm{~mm}$. This is expected to have just a minor impact on the results given that the drop remains small enough compared to the horizontal extent of the plate.

At the top of the plate ( $z=0$, see Fig. 7.1), two zones are distinguished depending on the position with respect to the patching point. Right below the drop $\left(0<r \leqslant R_{\mathrm{p}}\right)$, the heat flux lost by the substrate is equal to the heat flux consumed by the drop for its evaporation, $\mathscr{J} L=k_{\mathrm{v}}(r)\left(T_{\mathrm{S} \Sigma}(r)-T_{\mathrm{sat}}\right) / h(r)$. Outside the "patching perimeter" $\left(R_{\mathrm{p}}<r<R_{\mathrm{S}}\right)$, the heat loss by the substrate is due to natural convection in the air, here described by Newton's law of cooling $\alpha_{\mathrm{conv}}\left(T_{\mathrm{s} \Sigma}(r)-T_{\infty}\right)$, where $T_{\infty}$ is the ambient temperature far from the plate and $\alpha_{\text {conv }}$ is the convective heat transfer coefficient. The value of $\alpha_{\text {conv }}$ is determined from the experiments in the absence of the drop as $\alpha_{\text {conv }}=28 \mathrm{~W} / \mathrm{m}^{2} \mathrm{~K}$ (see subsection 7.4.2). Clearly, the heat distribution defined in this way flux generally proves to be discontinuous at $r=R_{\mathrm{p}}$, and is thus heuristically smoothened by adopting the following continuous form:

$$
-\left.k_{\mathrm{s}} \partial_{z} T_{\mathrm{s}}\right|_{z=0}=\left\{\begin{array}{cc}
k_{\mathrm{V}}(r) \frac{T_{\mathrm{s}}(r)-T_{\mathrm{sat}}}{h(r)} & \text { for } r \leq R_{p} \\
\alpha_{\text {conv }}\left(T_{\mathrm{s}}(r)-T_{\infty}\right)\left(1-e^{B\left(R_{\mathrm{p}}-r\right)}\right) \cdots & \\
\quad+\left(k_{\mathrm{v}}\left(R_{\mathrm{p}}\right) \frac{T_{\mathrm{s}}\left(R_{\mathrm{p}}\right)-T_{\mathrm{sat}}}{h\left(R_{\mathrm{p}}\right)}\right) e^{B\left(R_{\mathrm{p}}-r\right)} & \text { for } r>R_{p}
\end{array}\right.
$$

where $B$ is a parameter whose value is determined by demanding in addition continuous differentiability at $r=R_{\mathrm{p}}$.

The problem given by Equation 7.4-7.6 with the formulated boundary conditions is discretized in a standard way by second-order finite differences. The resulting nonlinear algebraic system of equations for the values of $h, \kappa, T_{\mathrm{S}}$ at the grid points as well as the values of $z_{\text {top }}$ and $B$ is solved by the Newton-Raphson method. It is checked a posterior $i$ that the choice of the patching point $R_{\mathrm{p}}$ has no significant influence on the results.

Finally, one has to note that, aside the above presented "full" model, a "partial" modelling will also be tested here. It consists in skipping the computation of the substrate temperature field and rather borrowing the measured substrate temperature distribution $T_{\mathrm{S} \Sigma}(r)$ from the experiment, using it directly in in order to be used in Equation 7.4 for the calculation of the vapour film thickness profile.

\subsection{Results and Discussion}

\subsubsection{Experimental observations and numerical validation}

Figure 7.4 shows the temperature fields inside the quartz plate for four different drop sizes and for an imposed temperature at the plate bottom $T_{\mathrm{imp}}=330^{\circ} \mathrm{C}$. For each size, the left half of the diagram shows the numerical results while the right half corresponds to the experimental data. A strong cooling of the substrate underneath the Leidenfrost drop is observed. The cooling proves to be roughly one third of the ideal superheat $T_{\mathrm{imp}}-T_{\mathrm{sat}} \simeq 150 \mathrm{~K}$, and generally depends on the heater temperature. The temperature 
field in the plate also depends on the drop size, as observed in Fig. 7.4. As the drop becomes larger, the global shape of the temperature field appears to change from a rather spherical shape in the drop vicinity into a rather one-dimensional field (as will be discussed in more detail in subsection 7.4.2).

The experimental and numerical results appear to compare rather well as highlighted by the iso-temperature contours in Fig. 7.4 and the temperature profiles at the top of the plate in Fig. 7.5a. In the latter figure, even if the agreement is overall quite satisfactory, the data reveal a systematic mismatch of $5 \%$ between experimental and numerical results and further deviate in the centre. In particular, the numerically predicted maximum substrate cooling (with respect to $T_{\mathrm{imp}}=330^{\circ} \mathrm{C}$ ) is here about $80 \mathrm{~K}$, while the measured one is about $95 \mathrm{~K}$. On the one hand, the mentioned mismatch can partly be attributed to the uncertainty in our calibration. On the other hand, the deviation in the center may further be related to oscillations of the Leidenfrost drops, which are actually always present in our experiments and likely to give rise to higher evaporation rates, and hence to stronger substrate cooling. Besides, evaporation modelling based only on the vapour film (ignoring the contribution of the upper part of the drop), even if it indeed remains the principal contribution, is nonetheless known to systematically underestimate the global evaporation cooling rates $[4,111]$.

At the same time, this moderate discrepancy is not deemed to be due to the presence of the needle in the experiment (and the absence thereof in the modelling), for a numerical study revealed that the bottom shape of the drop is barely affected by the presence of the needle for a given drop radius (as viewed from the top) in the range considered here (see section 7.E). It is interesting to note that in our studied parameter regime the minimum substrate temperature due to the cooling is practically not affected by the drop size, neither experimentally nor theoretically. Moreover, the theoretical results highlight that the substrate surface temperature drop is maximal right below the neck, i.e. at the location where the vapour film is the thinnest. This feature is apparently gummed out in the extraction of the experimental temperature profiles at the substrate surface but can still be observed from Fig. 7.4 in terms of the heat flux, which at the top of the substrate indeed proves to be maximum just at the neck location.

The cooling of the substrate due to the presence of a Leidenfrost drop is expected to affect the vapour film underneath the drop as compared to the corresponding isothermal substrate situation. As the cooling implies $T_{\mathrm{s} \Sigma}<T_{\mathrm{imp}}$, with the local superheat $T_{\mathrm{s} \Sigma}-T_{\mathrm{sat}}$ then being lower than its ideal value $T_{\mathrm{imp}}-T_{\mathrm{sat}}$, one can expect a reduction in both the evaporation rates and vapour film thicknesses (and hence an increase in the lifetime of the drops). This can already be qualitatively confirmed based on the scaling laws established for isothermal substrates [16], according to which $h \sim\left(T_{\mathrm{s} \Sigma}-T_{\mathrm{sat}}\right)^{1 / 3}$ in the neck region and $h \sim\left(T_{\mathrm{s} \Sigma}-T_{\mathrm{sat}}\right)^{1 / 6}$ in the main vapour pocket, both tending to decrease with reducing the superheat indeed. The global evaporation rate of the drop, $(-\dot{M})$, as determined principally by evaporation through the vapour film, is given by $(-\dot{M})=\frac{2 \pi}{L} \int_{0}^{R_{\mathrm{p}}} \frac{k_{\mathrm{v}}\left(T_{\mathrm{s} \Sigma}-T_{\mathrm{sat}}\right)}{h} r \mathrm{~d} r$, and is then also expected to decrease. The relatively small exponents in the mentioned scaling laws indicate that the vapour film thickness is apparently not modified too drastically as compared to the isothermal-substrate case, but a more essential effect can be expected, quite conversely, in terms of the evaporation rates. These speculations are partly confirmed in Fig. 7.5b that shows the numerically predicted profiles of the vapour layer for the cases considered in Fig. 7.4. The results 


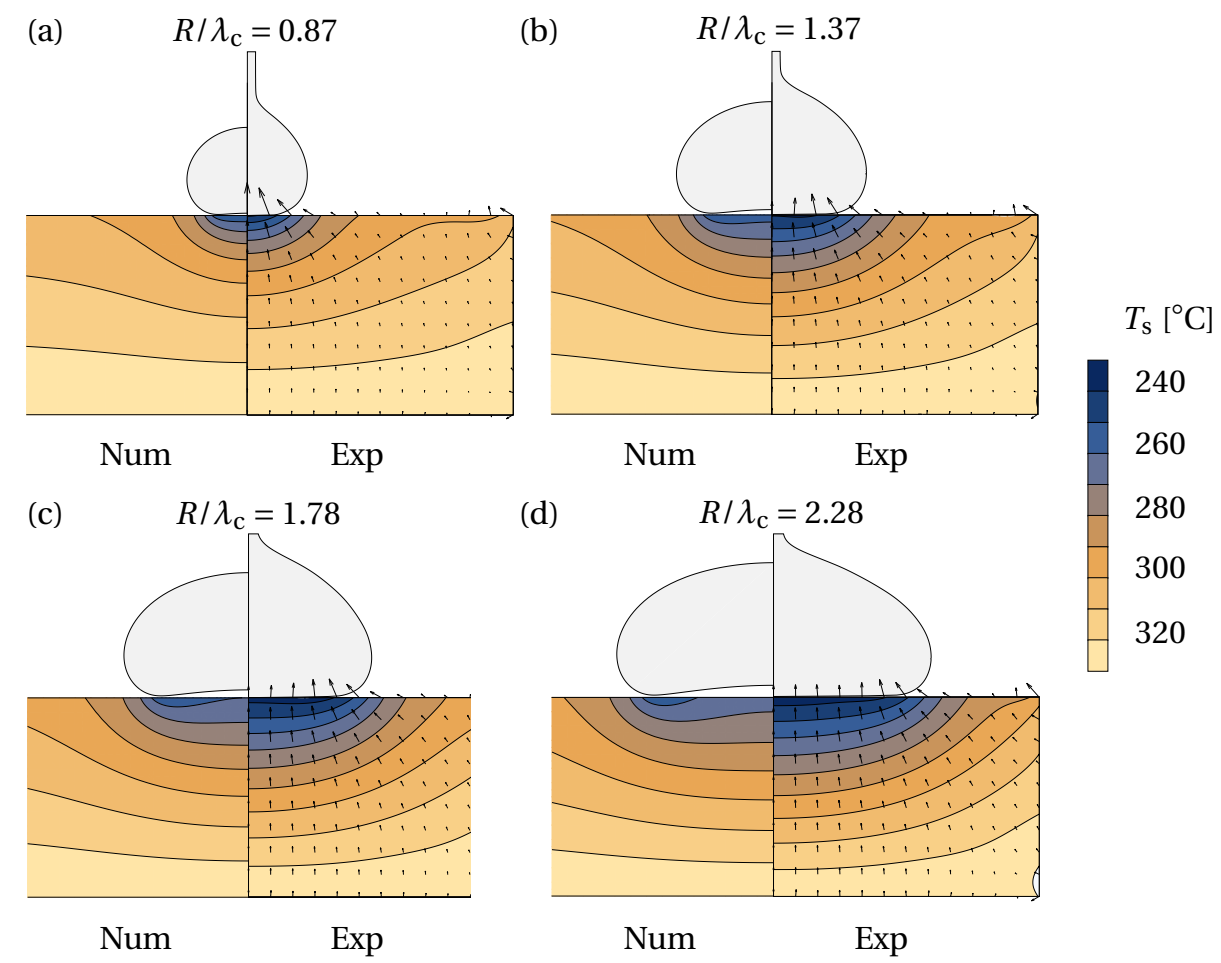

Figure 7.4: Temperature field inside the quartz plate under static Leidenfrost drops of ethanol of different sizes. For each presented cases, the numerical result (left half part) is juxtaposed with the experimental measurement (right half part) for comparison. On the latter, the arrows indicate the direction of the heat flux. The imposed temperature at the bottom of the substrate is $T_{\mathrm{imp}}=330^{\circ} \mathrm{C}$. The lengths are normalised with the capillary length $\lambda_{\mathrm{c}}$, i.e. $1.56 \mathrm{~mm}$ for ethanol at $T_{\mathrm{sat}}$. 
of the full model (dot-dashed lines) are compared to the corresponding results for an isothermal substrate with $T_{\mathrm{s} \Sigma} \equiv T_{\mathrm{imp}}$ (dashed lines). The results obtained by considering in the model the experimental temperature profile at the top of the substrate instead of fully solving for the temperature field in the plate are also provided (solid lines). One observes a close agreement between the full model and the one based on $T_{\mathrm{s} \Sigma}$ adopted from the experiment. In contrast, these numerical and "experimental" vapour film thicknesses manifest quite an appreciable reduction of about $17 \%$ relative to the isothermal-substrate case. As far as the global evaporation rates are concerned, the reduction is estimated to be about $26 \%$, which points to the potential importance of incorporating the effect of cooling into Leidenfrost modelling for not so perfectly thermally conductive substrates.

\subsubsection{Cooling strength criterion}

As already mentioned in section 7.3, the measurement of the temperature field in our quartz plate in the absence of any drop enables estimating the value of the heat transfer coefficient $\alpha_{\text {conv }}$ assuming heat losses at the top of the plate to be describable by Newton's cooling law $\alpha_{\text {conv }}\left(T_{\mathrm{s} \Sigma}-T_{\infty}\right)$. Whether or not any significant temperature gradient emerges in the plate as a result can be characterized by an appropriate Biot number (essentially a dimensionless form of the heat transfer coefficient). The heat balance across the plate can be written as

$$
T_{\mathrm{imp}}-T_{\mathrm{s} \Sigma}=\frac{\alpha_{\mathrm{conv}} H_{\mathrm{s}}}{k_{\mathrm{s}}}\left(T_{\mathrm{s} \Sigma}-T_{\infty}\right) \equiv \mathscr{B} i_{\infty}\left(T_{\mathrm{s} \Sigma}-T_{\infty}\right),
$$

where $\mathscr{B} i_{\infty}$ is the Biot number due to heat losses to the surroundings. Hence

$$
T_{\mathrm{s} \Sigma}=\frac{T_{\mathrm{imp}}+\mathscr{B} i_{\infty} T_{\infty}}{1+\mathscr{B} i_{\infty}}
$$

The smaller $\mathscr{B} i_{\infty}$, the more the heat transfer through the plate is limited by heat exchange with the surroundings without any noticeable temperature difference in the plate. It is interesting to note that, in principle, one cannot here go too far into the opposite limit of extremely poor thermal conducting substrates $\left(\mathscr{B} i_{\infty} \gg 1\right)$ due to the risk of $T_{\mathrm{S \Sigma}}$ actually falling below $T_{\mathrm{sat}}=79^{\circ} \mathrm{C}$ (for ethanol) and thus effectively killing the superheating. In our experimental setup, with $k_{\mathrm{s}}=1.4 \mathrm{~W} /(\mathrm{m} \mathrm{K}), H_{\mathrm{s}}=4.5 \mathrm{~mm}, T_{\mathrm{imp}}=330^{\circ} \mathrm{C}$ and $T_{\infty}=22^{\circ} \mathrm{C}, T_{\mathrm{imp}}-T_{\mathrm{s} \Sigma}=30 \mathrm{~K}$ was measured (in the absence of any drop). Thus, we obtain $\alpha_{\text {conv }} \approx 28 \mathrm{~W} /\left(\mathrm{m}^{2} \mathrm{~K}\right)$, and hence $\mathscr{B} i_{\infty} \approx 0.1$.

Following the same reasoning as above, but now in a largely estimative way for the case when the plate is exposed to an evaporating Leidenfrost drop, $\alpha_{\text {conv }}$ must be replaced by $k_{\mathrm{v}} / h, T_{\infty}$ by $T_{\mathrm{sat}}$, and $H_{\mathrm{s}}$ by $\min \left(H_{\mathrm{s}}, R\right)$. Here $\min \left(H_{\mathrm{s}}, R\right)$ is used to refleFct the fact (cf. Fig. 7.6) that in the vicinity of smaller drops $\left(R \ll H_{\mathrm{S}}\right)$ a more spherical temperature field is found, with the typical conduction length scale $R$. In contrast, for larger drops $\left(R>H_{\mathrm{s}}\right)$ the temperature field becomes predominantly one-dimensional in the vertical direction, with the typical length scale $H_{\mathrm{s}}$. Thus, we obtain an estimation

$$
T_{\mathrm{s \Sigma}} \sim \frac{T_{\mathrm{imp}}+\mathscr{B} i_{\mathrm{d}} T_{\mathrm{sat}}}{1+\mathscr{B} i_{\mathrm{d}}},
$$


(a)

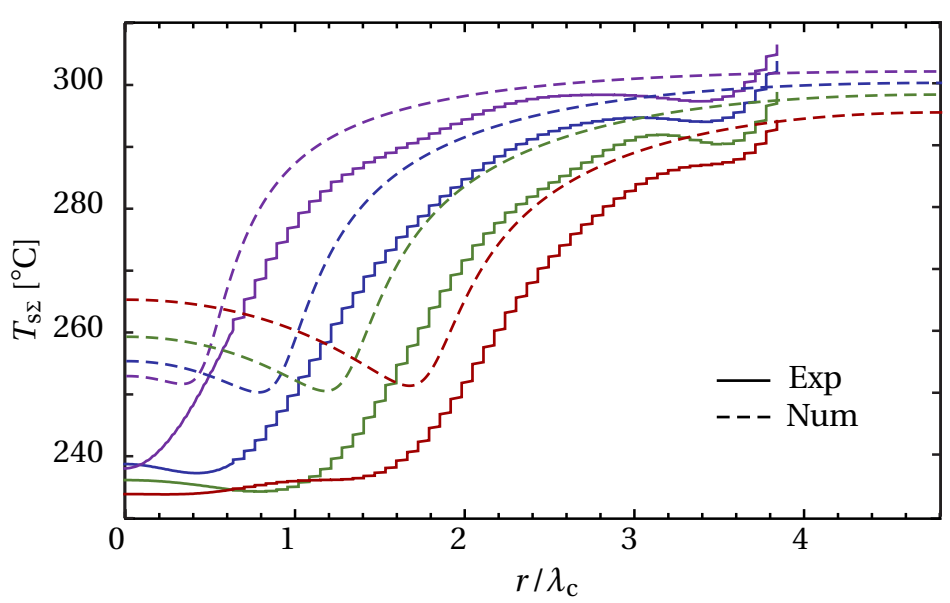

(b)

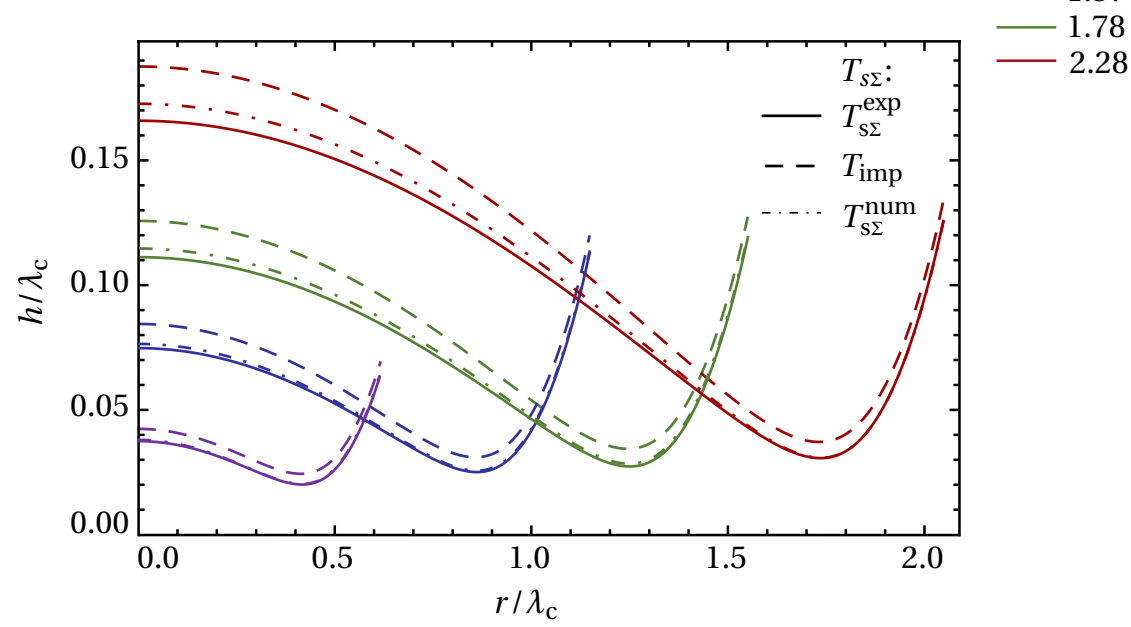

Figure 7.5: Profiles of the substrate surface temperature (a) and of the vapour film thickness (b) for $T_{\mathrm{imp}}=330^{\circ} \mathrm{C}$ and four different drop sizes. Three cases are shown in panel (b) for each drop size according to the way the substrate surface temperature profile is handled in the model: fully isothermal substrate at $T_{\mathrm{imp}}$ (dashed lines), borrowed from the experiment (solid lines), and calculated in the framework of the full model (dot-dashed lined). Panel (b) presents both experimental and numerical results, the latter calculated from the full model. 


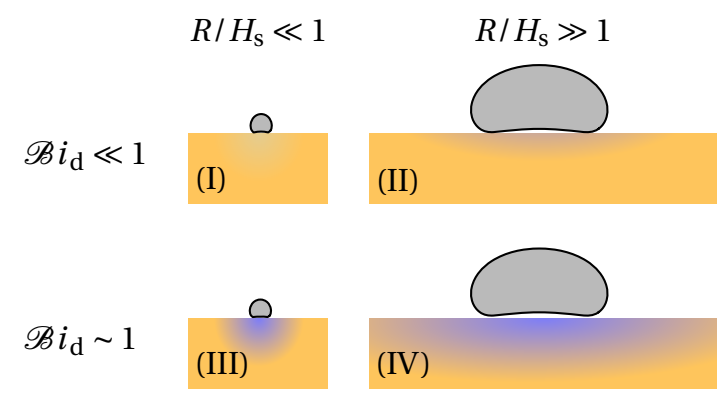

Figure 7.6: Schematic illustrating the four different regimes for the temperature field in the substrate underneath a Leidenfrost drop, which depend on the ratio between the drop radius $R$ and the plate thickness $H_{\mathrm{s}}$ as well as on the Biot number $\mathscr{B} i_{\mathrm{d}}$ (incorporating in itself the substrate thermal conductivity). Regimes (II) and (IV) are characterized by a more one-dimensional profile, while in the other limit, regimes (I) and (III) resemble locally a spherical profile from a point source. Since for regimes (III) and (IV) the Biot number is not small, a significant cooling by the evaporating drop is expected, while for regimes (I) and (II) the substrate remains largely isothermal.

for the substrate surface temperature underneath the drop, with

$$
\mathscr{B} i_{\mathrm{d}}=\frac{k_{\mathrm{v}} \min \left(H_{\mathrm{s}}, R\right)}{k_{\mathrm{s}} h},
$$

where $\mathscr{B} i_{\mathrm{d}}$ is the Biot number associated with the cooling due to the presence of the drop, which once again indicates whether a significant temperature variation in the substrate is present $\left(\mathscr{B} i_{\mathrm{d}} \sim 1\right)$ or not $\left(\mathscr{B} i_{\mathrm{d}} \ll 1\right)$, the isothermal substrate limit being obviously attained for $\mathscr{B} i_{\mathrm{d}} \rightarrow 0$.

Given the estimative character of $\mathscr{B} i_{d}$, we shall just use a typical vapour layer thickness $h_{\text {mean }}$ for $h$ in Equation 7.7, all the more so as these values do not vary very strongly with the superheat in the typical working range. For instance, in our setup, we have $h_{\text {mean }} \sim 50 \mu \mathrm{m}$ for $R=0.87 \lambda_{\mathrm{c}}$ and $h_{\text {mean }} \sim 130 \mu \mathrm{m}$ for $R=2.28 \lambda_{\mathrm{c}}$ at $T_{\mathrm{imp}}=330^{\circ} \mathrm{C}$. Thus, the ratio $R / h_{\text {mean }}$ is seen to be roughly constant and $\mathscr{B} i_{d} \sim 0.45$ for our quartz substrate irrespective of the drop size. On account of Fig. 7.4.2, this goes well along with the fact that the substrate cooling under the Leidenfrost drop proves to be almost identical in Fig. 7.5a whatever the drop size. Furthermore, with $\mathscr{B} i_{d}=0.45, T_{\text {sat }}=79^{\circ} \mathrm{C}$ and $T_{\mathrm{imp}}=330^{\circ} \mathrm{C}$, Fig. 7.4.2 yields $T_{s \Sigma} \sim 250^{\circ} \mathrm{C}$, which turns out to be a good estimation indeed (cf. Fig. 7.5a). At last, note that the substrate cooling due to the Leidenfrost drop is here greater than that due to heat losses to the environment quite in agreement with the estimation $\mathscr{B} i_{d}>\mathscr{B} i_{\infty}$ obtained in terms of the Biot numbers.

The four different regimes are now explored numerically. The temperature profile at the top surface of the substrate is presented in Fig. 7.7 for ethanol drops of $R=$ $0.4 \lambda_{\mathrm{c}}$ (solid lines) and 3.75 $\lambda_{\mathrm{c}}$ (dashed lines) and different thermal conductivities of the substrate $k_{\mathrm{s}}$. The temperature variation is seen to become less pronounced indeed for decreasing $\mathscr{B} i_{\mathrm{d}}$, while decreasing the radius results in a more spherical profile since $R / H_{\mathrm{s}}$ becomes smaller. 


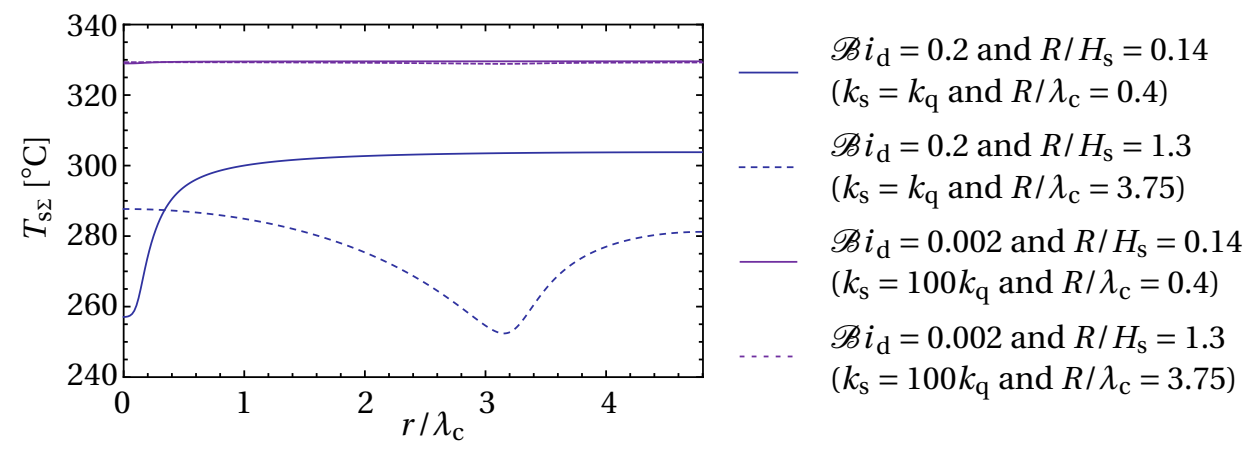

Figure 7.7: Numerically determined temperature profile at the top of a substrate underneath a Leidenfrost drop for different drop sizes and thermal conductivities of the substrate. $H_{\mathrm{s}}$ is taken at the value of the quartz plate thickness used in the experiment. $\mathscr{B} i_{\mathrm{d}}$ is calculated numerically using the area- averaged values of $h(r)$ and $k_{\mathrm{v}}(r)$ in the vapour layer.

\subsubsection{Exploring the influence of the substrate thermal conductivity}

We have seen that the strength of substrate cooling due to a Leidenfrost drop is greatly influenced by the thermal conductivity of the substrate $k_{\mathrm{s}}$. Therefore, to further explore this effect, we here carry out a parametric study by varying $k_{\mathrm{s}}$ beyond the constraints of a concrete experimental setup (in this sense modelling various substrate materials). As earlier in Fig. 7.5, we shall be interested in the profiles of both the substrate surface temperature and the vapour film thickness. It is natural to expect the cooling of the plate to be reduced as $k_{\mathrm{s}}$ is increased so that the isothermal limit is recovered for relatively high substrate thermal conductivities. Numerical results to test this hypothesis are shown in Fig. 7.8 for an ethanol drop of $R=1.37 \lambda_{\mathrm{c}}$. Indeed, increasing the thermal conductivity of the substrate is seen to rapidly lead to the isothermal regime. For $k_{\mathrm{s}}=10 k_{\mathrm{q}}$, the maximum cooling is reduced from about $75 \mathrm{~K}$ to only about $10 \mathrm{~K}$, while for $k_{\mathrm{s}} \gtrsim 100 k_{\mathrm{q}}$, no discernible cooling with respect to the imposed bottom temperature of the plate can be found within our numerical precision (panel a). With the increase in the substrate surface temperature, the evaporation rate increases as well, resulting in a thicker vapour layer as shown in the panel (b).

\subsubsection{Regression to the isothermal model}

We have seen that the finite thermal conductivity of the quartz plate has a significant influence on the temperature field under the drop. It is therefore interesting to compare the temperature fields and the vapour layer thickness with the results for an isothermal plate. We will compare the scaling behaviour for the vapour layer thickness with the scaling laws as established for the isothermal case. We give insight how to extends the framework of isothermal substrates to the case where the cooling of the plate has a significant influence on the evaporation process.

Figure 7.9 shows the numerically predicted profiles of the vapour layer thickness for several drop sizes and several imposed temperature. The results of the full model 
(a)

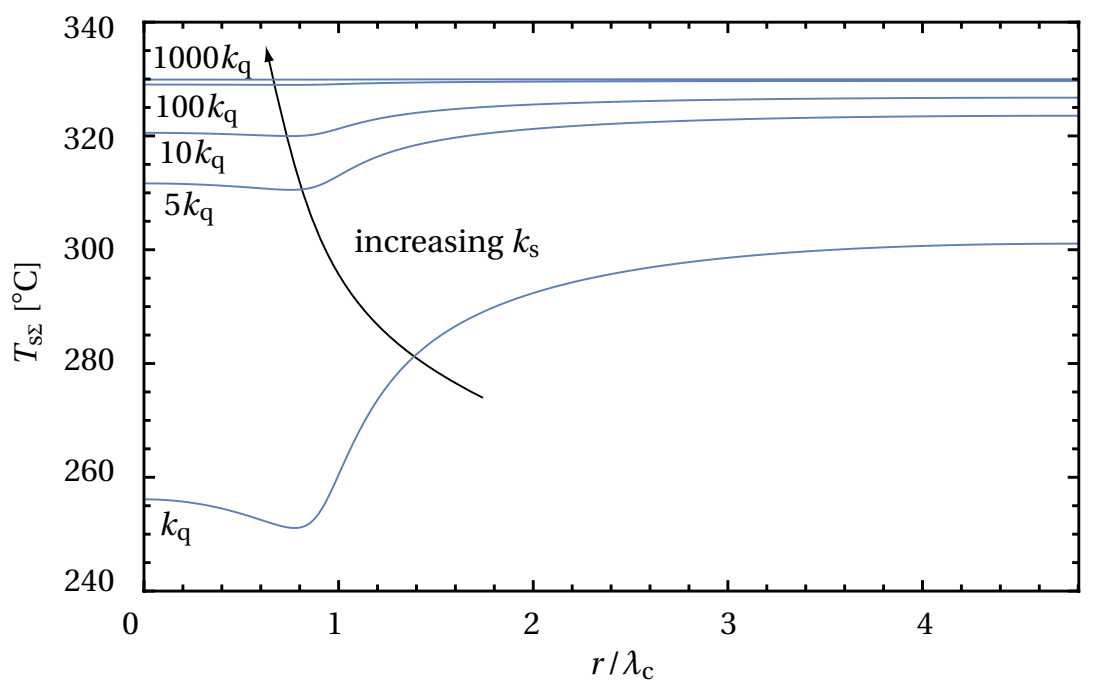

(b)

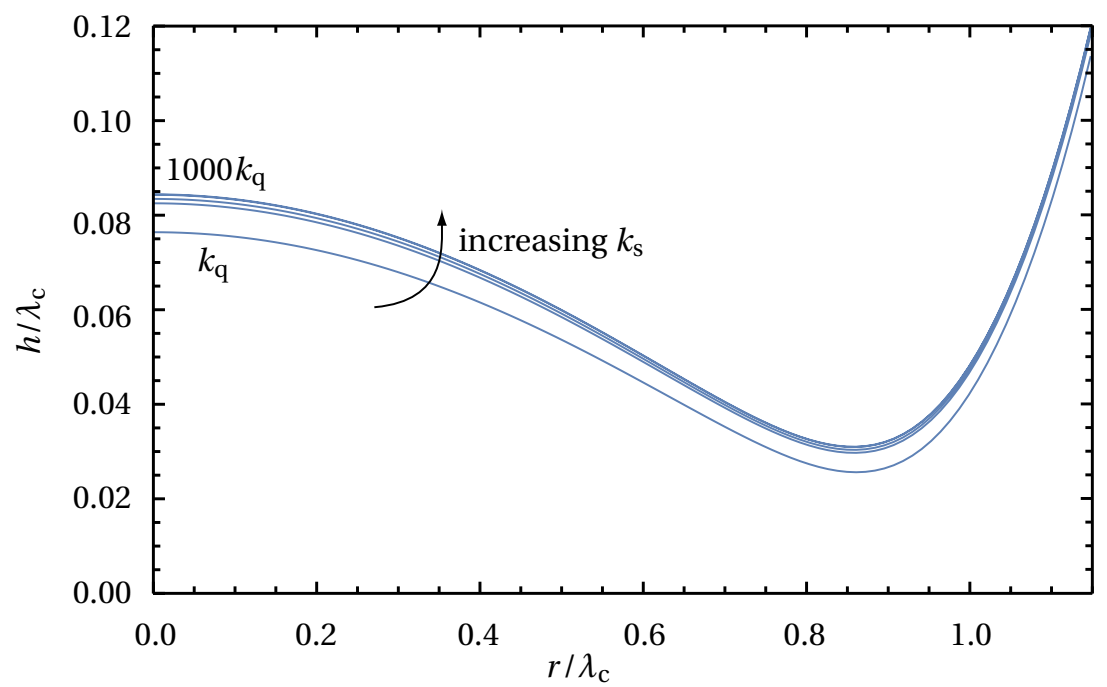

Figure 7.8: Effect of the variation of the substrate thermal conductivity $k_{\mathrm{s}}$ on the numerically predicted profiles of the substrate surface temperature (a) and the vapour film thickness (b) underneath a Leidenfrost ethanol drop of $R=1.37 \lambda_{\mathrm{c}}$ with $T_{\mathrm{imp}}=330^{\circ} \mathrm{C}$ and the plate thickness $\left(H_{\mathrm{s}}=4.5 \mathrm{~mm} \approx 3 \lambda_{\mathrm{c}}\right)$ as in the experiment. The value of $k_{\mathrm{s}}$ for the quartz used in the experiment is denoted by $k_{\mathrm{q}}=1.4 \mathrm{~W} /(\mathrm{mK})$. 


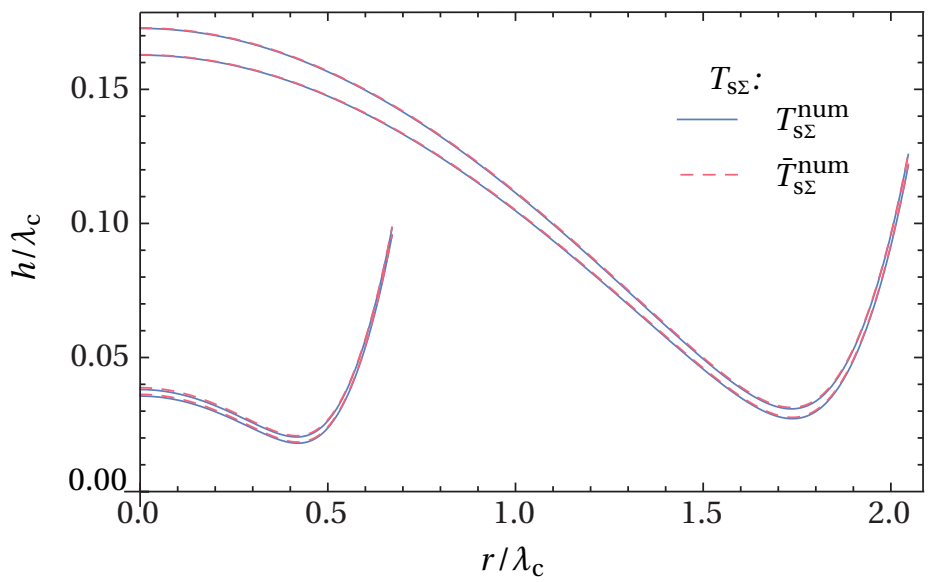

Figure 7.9: Numerically predicted profiles of the vapour film thickness for two drop sizes, $R=0.87 \lambda_{\mathrm{c}}$ and $R=2.28 \lambda_{\mathrm{c}}$, and two imposed temperature, $T_{\mathrm{imp}}=330^{\circ} \mathrm{C}$ (top) and $T_{\mathrm{imp}}=275^{\circ} \mathrm{C}$ (bottom). The results of the full model (solid lines) are compared to the corresponding results for an isothermal substrate at the average plate surface temperature below the drop calculating from the full model, i.e. $T_{\mathrm{s} \Sigma}=\bar{T}_{\mathrm{s} \Sigma}$ (dashed line).

(solid lines) are compared to the corresponding results for an isothermal substrate at the average plate surface temperature below the drop calculating from the full model, i.e. $T_{\mathrm{s} \Sigma}=\bar{T}_{\mathrm{s} \Sigma}$ (dashed line). One observes that the predicted shapes of the vapour layer are quasi-identical. It means that instead of solving the full model, it would be possible to solve just the isothermal-substrate model if the mentioned mean temperature could somehow be evaluated (e.g. like with Fig. 7.4.2 in subsection 7.4.2). This is attributed to relatively small (compared to the superheat) radial variations of $T_{\mathrm{s} \Sigma}(r)$ underneath the drop actually occurring in the present setup. Consequently, the results by Sobac et al. [16], obtained for isothermal substrates (viz. perfectly thermal conducting plates in the present context), are expected to be valid with a reasonable precision here even for poor thermal conducting substrates in terms of the mean temperature $\bar{T}_{\mathrm{s} \Sigma}$.

For instance, a number of scaling laws have been established by Sobac et al. [16] as far as the vapour film thicknesses and the evaporation rates are concerned, expressed as functions of an evaporation number $\mathscr{E}$. In particular, $h_{\text {neck }} \sim \mathscr{E}^{1 / 3}$ for the neck thickness, the neck being identified as the narrowest point of the vapour film, see e.g. Fig. 7.8b. In the present context, such an evaporation number can just be defined in a modified way, based on $\bar{T}_{\mathrm{s} \Sigma}$, as follows:

$$
\mathscr{E}=\frac{\bar{k}_{\mathrm{v}} \bar{\mu}_{\mathrm{v}}\left(\bar{T}_{\mathrm{s} \Sigma}-T_{\mathrm{sat}}\right)}{\gamma \bar{\rho}_{\mathrm{v}} \lambda_{\mathrm{c}} L},
$$

The vapour properties are here evaluated at $\left(\bar{T}_{\mathrm{s} \Sigma}+T_{\mathrm{sat}}\right) / 2$ (i.e. in a manner similar to section 7.3 [16]) to yield in particular $\bar{k}_{\mathrm{v}}, \bar{\mu}_{\mathrm{v}}$ and $\bar{\rho}_{\mathrm{v}}$. For example, as shown in Fig. 7.10, the neck thickness, computed generally from a non-isothermal substrate model (full or partial) observes well the mentioned $1 / 3$ power law in terms of the hereby 


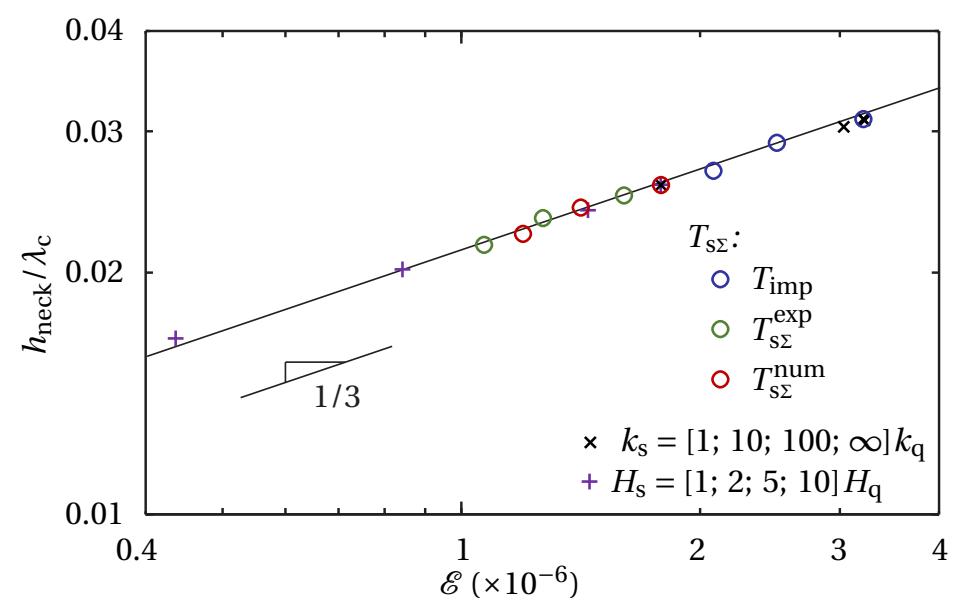

Figure 7.10: The 1/3 power law in terms of the evaporation number defined in Equation 7.8 for the numerically determined neck thickness. The three circles within each (blue, black and red) set correspond, in an increasing order of $\mathscr{E}$, to $T_{\text {imp }}=220^{\circ} \mathrm{C}$ and $R=1.39 \lambda_{\mathrm{c}}$, to $T_{\mathrm{imp}}=275^{\circ} \mathrm{C}$ and $R=1.314 \lambda_{\mathrm{c}}$, and to $T_{\mathrm{imp}}=330^{\circ} \mathrm{C}$ and $R=1.37 \lambda_{\mathrm{c}}$, a slight scattering in the chosen $R$ values being due to the one in corresponding experimental realisations. The colours: blue for the overall isothermal substrates at $T_{\mathrm{imp}}$, black for the partial model (with experimentally measured temperature profiles), and red for the full model. Crosses and pluses all correspond to the full model at $T_{\mathrm{imp}}=330^{\circ} \mathrm{C}$ and $R=1.37 \lambda_{\mathrm{c}}$. The crosses are for $H_{\mathrm{s}}=H_{\mathrm{q}}$ and the indicated values of $k_{s}$, while the pluses are for $k_{\mathrm{s}}=k_{\mathrm{q}}$ and the indicated values of $H_{\mathrm{s}}$. Here $H_{q}=4.5 \mathrm{~mm}$ and $k_{\mathrm{q}}=1.4 \mathrm{~W} /(\mathrm{mK})$ are the $H_{\mathrm{s}}$ and $k_{\mathrm{s}}$ values of the quartz plate used in the experiments.

defined parameter $\mathscr{E}$ whatever the substrate conductivity, the substrate thickness and the imposed temperature. A slight scattering of points about the straight line is mainly attributed to slightly different values of $R$ used in the computation following different experimental runs (for which it is difficult to reproduce exactly the same $R$ ), for the proportionality factor is actually $R$-dependent [16].

\subsection{Conclusions}

The present study has focused, both experimentally and theoretically, on the temperature field in the solid substrate underneath a Leidenfrost drop in the case of relatively poor thermal conducting solids such as quartz. A non-trivial feedback between substrate cooling and Leidenfrost characteristics has been highlighted. In the experiments, the drop is continuously fed from a needle so as to achieve an overall stationary situation. The temperature field in a quartz plate substrate is experimentally visualised by means of a Mach-Zehnder interferometer. Quite a strong local cooling of the substrate caused by a Leidenfrost (ethanol) drop was detected, which comes alongside with a non-negligible cooling due to natural convection in the air already present in the absence of any drop. For instance, a local temperature drop as large as $80 \mathrm{~K}$ was measured 
in the range of parameters investigated in this study (viz. a $4.5 \mathrm{~mm}$ thick quartz plate with its bottom surface maintained at $330^{\circ} \mathrm{C}$ ). This can be compared with a $30 \mathrm{~K}$ upper surface cooling measured in the absence of any drop, and with the intended "ideal" superheat of $251 \mathrm{~K}\left(=330^{\circ} \mathrm{C}-79^{\circ} \mathrm{C}\right)$, the ethanol boiling temperature being equal to $79^{\circ} \mathrm{C}$ ). Quite remarkably, such a maximum local cooling proved to depend only weakly on the drop size in a capillary-length range. A theoretical model has been developed in order to rationalise the experimental data and it appears to fairly well compare with the experiments, despites its simplicity. Interestingly enough, the simulations revealed the maximum substrate temperature drop to occur right underneath the neck of the vapour film, roughly coinciding with the expected maximum of heat (and evaporation) fluxes, although the experimental resolution did not prove to be sufficient to conclusively capture such a fine effect in terms of the temperature drop (though it did in terms of the local heat flux). On the other hand, the modelling somewhat underestimates (by some $15 \mathrm{~K}$ ) the value of the temperature drop, which apparently partly goes along with a long-standing problem of underestimating the overall evaporation rates when not accounting for the contribution of the upper part of the drop, even if the contribution of the lower part (i.e. the vapour film) is indeed predominant. The simulations showed the substrate cooling to be associated with a reduction of about $17 \%$ in the vapour film thickness and $26 \%$ in the evaporation rate as compared with the case of an isothermal substrate (at $330^{\circ} \mathrm{C}$ ) under otherwise equal settings. Thus, the Leidenfrost effect is subject to an appreciable influence of substrate non-isothermia. A parametric study was carried out in order to explore what happens for substrates with properties (thermal conductivity, size) beyond the constrains of the present experimental settings. Quite expectedly, as the thermal conductivity is increased, the isothermal substrate case is recovered, for which a Leidenfrost model like the earlier one by Sobac et al. [16] becomes fully sufficient. Simple criteria in terms of Biot numbers were formulated permitting to $a$ priori estimate the expected importance of substrate cooling for any particular settings. Finally, whatever the value of the Biot number, the scaling laws for the vapour film thickness as a function of the evaporation number established elsewhere in the case of an isothermal substrate prove to remain valid here with a good accuracy if for the isothermal substrate temperature one substitutes therein the mean substrate surface temperature underneath the Leidenfrost drop. 


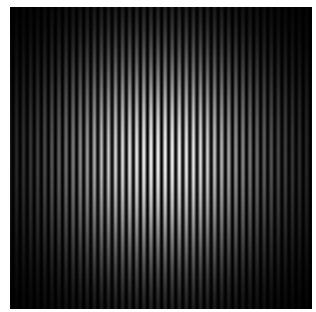

(a)

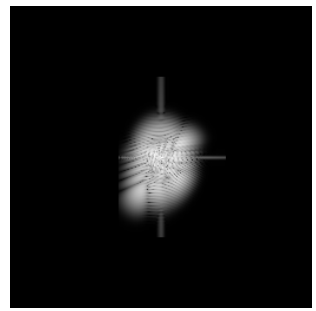

(d)

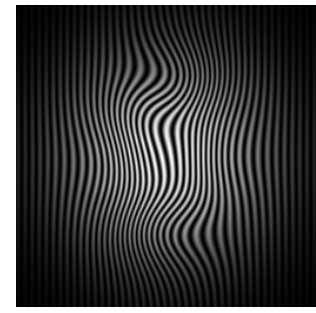

(b)

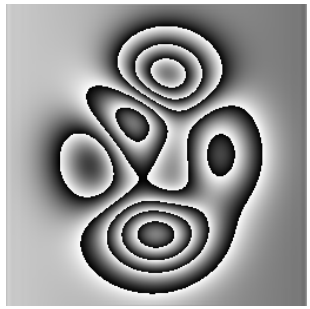

(e)

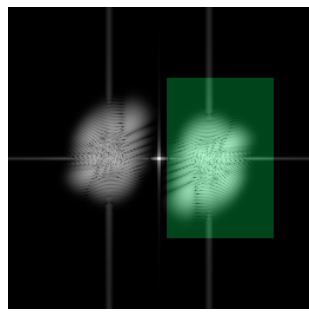

(c)

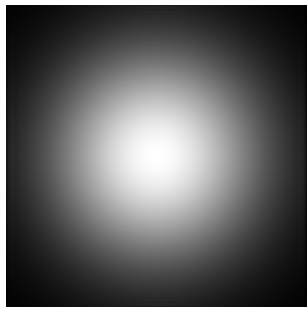

(f)

Figure 7.11: Demonstration of the phase extraction process with a computer generated interferogram. The generated finite fringe inteferogram (a) is distorted (b) and analysed by a Fourier filtering method (c) and (d), recovering the phase (e) and magnitude (f) of the distortion.

\section{A Phase extraction}

The extraction of the phase from our experimental involves several steps which will be explained here. We will highlight the advantages of using the finite-fringe method in conjunction with Fourier filtering to reduce influences from experimental noise.

The first processing step is the decomposition of the interferogram in its (wrapped) phase and magnitude components using a Fourier transform technique (Fig. 7.11), which eliminates low frequency background variations and high frequency noise from the interference phase $[112,113]$. For a uniform refractive index field, the finite fridge width interferogram consists of evenly spaced interference fringes with a Gaussian intensity modulation 7.11a. Additional phase variations result in distortion of this fringe pattern 7.11b. To extract the phase, the two-dimensional Fourier spectrum is computed 7.11c and a rectangular window is applied (the green shaded area). The window only keeps a part of one of the half planes corresponding with the modulation of carrier fringes. The carrier phase is then removed by recentering the windowed spectrum to the carrier phase frequency $7.11 \mathrm{~d}$. The same spectrum recentering translation has to be used for the reference and test interferograms. Therefore, we recenter the spectrum for the reference interferogram first and use the same translation for subsequent interferograms. Finally, an inverse Fourier transformation yields the interference phase modulo $2 \pi 7.11 \mathrm{e}$ and magnitude $7.11 \mathrm{f}$ corresponding with the interferogram $7.11 \mathrm{~b}$. 
(a)

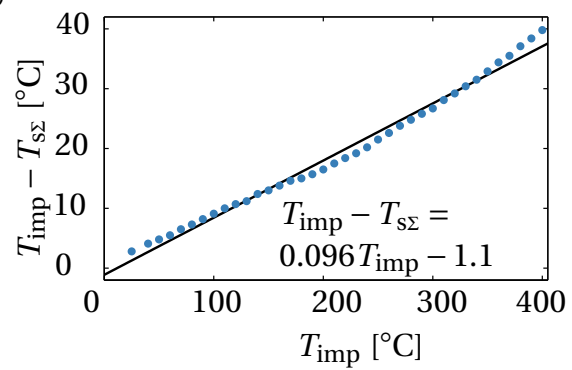

(b)

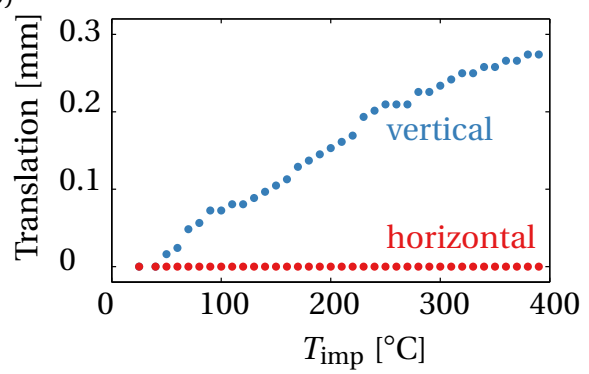

Figure 7.12: Temperature difference $\Delta T=T_{\mathrm{imp}}-T_{\mathrm{s} \Sigma}$ across the quartz substrate (a) and the apparent translation relative to the reference interferogram (b).

\section{B Calibration of $\mathrm{d} n / \mathrm{d} T$ and refraction correction}

Since the conversion from the optical path length difference into temperature fields involves careful calibration, here we will describe the steps taken to ensure the reliability of our experimental results.

The temperature at the top and bottom of the substrate were measured with a surface probe (Anritsu Meter Co., Ltd. N-141K-02-1-TC1-ANP) and a thermocouple inside the heater, respectively. First, a reference interferogram was recorded at room temperature $T_{0}=22^{\circ} \mathrm{C}$ to extract phase and magnitude corresponding with an isothermal substrate. Next, for every increase of $10^{\circ} \mathrm{C}$ in heater temperature setpoint $T_{\mathrm{imp}}$, an interferogram was recorded and the temperature was measured at the top of the quartz top obtain $\Delta T=T_{\mathrm{imp}}-T_{\mathrm{s} \Sigma}$, which is plotted in Fig. 7.12a. With this increasing temperature gradient the apparent translation due to refraction increases too (Fig. 7.12b). This translation is corrected for before phase subtraction and magnitude division.

Fig. 7.13 shows phases extraction of interferograms recorded at room temperature and with the heater at $400{ }^{\circ} \mathrm{C}$. The phase map in $7.13 \mathrm{c}$ shows that the index gradient in the air above the quartz is negligible compared to the index gradient inside the quartz. This supports the assumption that the refractive index change due to the heating of air is negligible compared to the refractive index change inside the quartz. Next, the phase is unwrapped ( $7.13 \mathrm{~d})$. 7.13e shows the vertical profiles corresponding with the region indicated by the rectangle in $7.13 \mathrm{~d}$. An offset $\phi_{\mathrm{h}}$ is added such that the phase at the heater surface corresponds with the temperature difference between the heater temperature and room temperature,

$$
\phi_{\mathrm{h}}=\frac{2 \pi d\left(T_{\mathrm{h}}-T_{0}\right)}{\lambda} \frac{d n}{d T} .
$$

Unwrapping errors are encountered near the top and bottom edge of the quartz. Therefore, the phase profiles are extrapolated towards the top and bottom surface using a linear fit between 0.5 and $1 \mathrm{~mm}$ from either surface. The phase difference $\Delta \phi=\phi_{\mathrm{h}}-\phi_{\mathrm{s}}$ is then computed from the extrapolated values, $\phi_{\mathrm{h}}$ at the heater surface and $\phi_{\mathrm{s}}$ at the quartz surface. From (7.1), it follows that the index difference $\Delta n$ (relative to the reference beam, averaged over the substrate thickness $d$ in the line-of-sight 


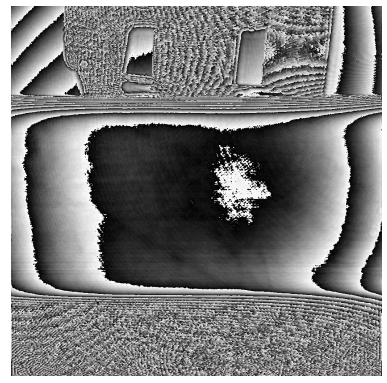

(a)

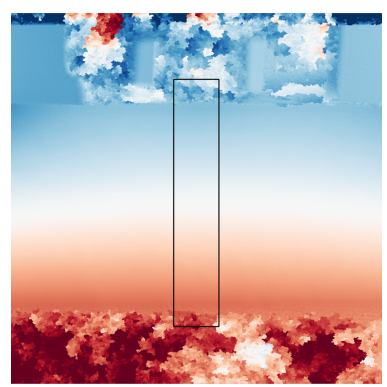

(d)

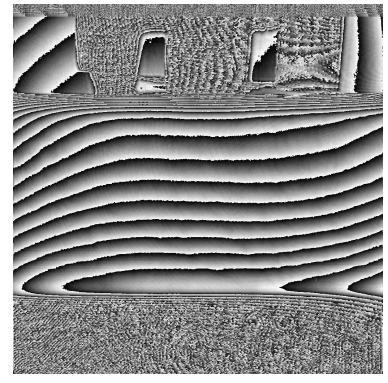

(b)

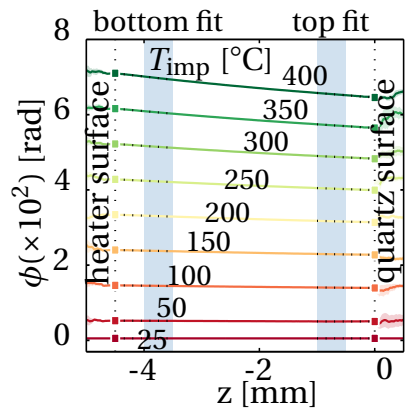

(e)

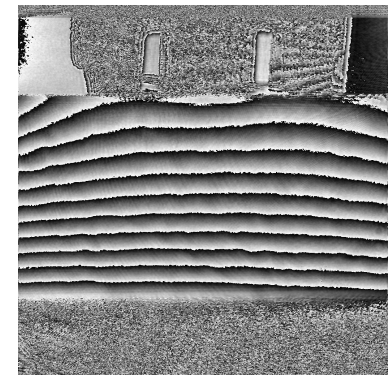

(c)

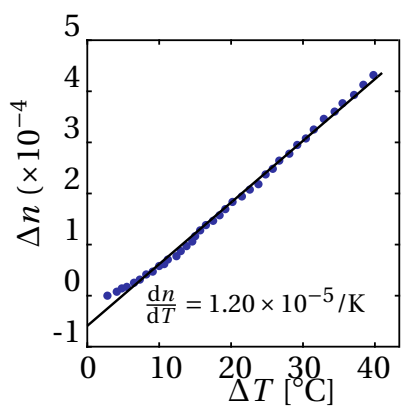

(f)

Figure 7.13: Calibration phase and magnitude extraction. Top row: phase modulo $2 \pi$ taken from a reference interferogram at $22^{\circ} \mathrm{C}$ (a) and at $400{ }^{\circ} \mathrm{C}$ (b) together with the their difference (c) (black and white corresponds to $-\pi$ and $\pi$, respectively). Image (d) shows the image after unwrapping (blue and red corresponds with a phase difference of $40 \pi$ ), where the rectangle corresponds with the area that is used to determine the vertical phase profile. Results for $\phi(z)$ for different $T_{\text {imp }}(\mathrm{e})$ to obtain $\Delta n(\Delta T)$ and the linear fit for $\mathrm{d} n / \mathrm{d} T$ (f). The lines and shading in (e) correspond with the mean and standard deviation in the horizontal direction of the rectangle in (d), respectively. The phase in the shaded area is extrapolated towards the heater and the quartz surface. 
direction) is

$$
\Delta n=\frac{\lambda \Delta \phi}{2 \pi d} .
$$

Now, $\mathrm{d} n / \mathrm{d} T$ can be determined from a linear fit of $\Delta n$ as function of $\Delta T$ ( 7.13f). The result, $\mathrm{d} n / \mathrm{d} T=(1.20 \pm 0.01) \times 10^{-5} / \mathrm{K}$, is consistent with the values reported in the literature [94, 107].

For the Leidenfrost experiments, the heater can now be set to any temperature $T_{\mathrm{h}}$ to record an interferogram with and without drop recorded. The phase difference between these two then gives the amount of cooling due to the drop. The phase contribution due to the convective cooling of the substrate without a drop is added from an interpolation of the phase profiles from 7.13e.

\section{C Abel inversion}

Details on our novel Abel inversion are presented in the following section. Our method is based on fitting the data using the projection on a basis function expansion. The experimental data is thus fitted in Abel-space where the found constants thus describe also the temperature field.

The interferometric phase $\phi(y, z)$ is a $2 \mathrm{D}$ projection of the refractive index field $\Delta n(x, y, z)$, The underlying field must therefore be determined from the projection by tomographic reconstruction. If the underlying field is axisymmetric, a single projection $\phi(y, z)$ is sufficient to reconstruct $\Delta n(r, z)$ (in cylindrical coordinates). The projection is then related to the field by the Abel transform:

$$
\begin{aligned}
F(y, z) & =\int_{-\infty}^{\infty} f(r, z) d x, \quad r=\sqrt{x^{2}+y^{2}} \\
& =2 \int_{y}^{\infty} \frac{f(r, z) r}{\sqrt{r^{2}-y^{2}}} d r
\end{aligned}
$$

where in our case

$$
\begin{aligned}
F(y, z) & =\Delta \phi(y, z), \\
f(r, z) & =\frac{2 \pi \Delta n(r, z)}{\lambda} .
\end{aligned}
$$

The analytic Abel inversion is given by

$$
f(r, z)=-\frac{1}{\pi} \int_{r}^{\infty} \frac{\partial F(y, z)}{\partial y} \frac{1}{\sqrt{y^{2}-r^{2}}} d y .
$$

However, this integral is sensitive to noise, especially near the axis of symmetry [114]. In order to reliably extract the local heat flux from the temperature field, we propose an inversion method based on the basis function expansion method (BASEX) which imposes that the reconstructed temperature field satisfies the Laplace equation for steady heat conduction. With BASEX, the unknown distribution $f(r, z)$ is approximated by a complete set of functions with linear coefficients [109]. The phase projection of this expansion solution (the forward Abel transform) is computed by numerical integration. 
The coefficients are then obtained by matching coefficients of the projection to the measured projection data $F(y, z)$ using linear regression. Based on physical arguments given in subsection 7.2.1, our domain is restricted to the width $W$ of the quartz plate, thus (7.11) becomes a definite integral:

$$
F(y, z)=\int_{-W / 2}^{W / 2} f(r, z) d x
$$

The axi-symmetric solution to the Laplace equation $\nabla^{2} f=0$ in cylindrical coordinates is given by the family of functions [115]

$$
f_{m k}(r, \theta, z)=\sum_{p=1}^{N}\left(A_{\mathrm{p}} J_{0}(k r)+B_{\mathrm{p}} Y_{0}(k r)\right) e^{ \pm k z}
$$

where $A_{\mathrm{p}}$ and $B_{\mathrm{p}}$ are constants, $k$ is a real or imaginary number and $J_{0}(r)$ and $Y_{0}(r)$ are Bessel functions of first and second kind, respectively. Since $Y_{0}(k r)$ diverges for $r \rightarrow 0$, we must have $B=0$. The remaining functions $f_{k}(r, z)=\sum_{p=1}^{N} A_{\mathrm{p}} J_{0}(k r) e^{ \pm k z}$ can be divided in two families of solutions. The first family provides a complete orthogonal set of functions on a disc of radius $a$ in the form of a Fourier-Bessel series:

$$
f(r, z)=\sum_{p=1}^{\infty}\left[A_{\mathrm{p}} \sinh \left(x_{\mathrm{p}} z / a\right)+B_{\mathrm{p}} \cosh \left(x_{\mathrm{p}} z / a\right)\right] J_{0}\left(x_{\mathrm{p}} r / a\right)
$$

where $k=\zeta_{\mathrm{p}} / a$ is real, with $\zeta_{\mathrm{p}}$ the $p$ th root of the zeroth order Bessel function of the first kind. The second family provides a complete orthogonal set of functions on a surface at constant $r$ with $0 \leq z \leq c$ in the form of a Fourier series:

$$
f(r, z)=\sum_{p=1}^{\infty}\left[C_{\mathrm{p}} \sin (p \pi z / c)+D_{\mathrm{p}} \cos (p \pi z / c)\right] I_{0}(p \pi r / c)
$$

where $k=i p \pi r / c$ is imaginary with $p=1,2, \ldots$ and $I_{0}(k r)=J_{0}(i k r)$ is the zeroth order modified Bessel function of the first kind. In our case, we take $c=H_{s}$, and add a translation in the $\mathrm{z}$-direction such that $\mathrm{z}=0$ at the top of the substrate for the measured data.

To approximate the 3D field $\Delta n(r, z)$, we use a linear combination of both families plus an offset term $f_{0}$ :

$$
\begin{aligned}
f(r, z)=f_{0}+\sum_{p=1}^{N}\left[A_{\mathrm{p}} \sinh \left(\zeta_{\mathrm{p}} z / a\right)\right. & \left.+B_{\mathrm{p}} \cosh \left(\zeta_{\mathrm{p}} z / a\right)\right] J_{0}\left(\zeta_{\mathrm{p}} r / a\right) \\
& +\sum_{p=1}^{M}\left[C_{\mathrm{p}} \sin (p \pi z / c)+D_{\mathrm{p}} \cos (p \pi z / c)\right] I_{0}(p \pi r / c) .
\end{aligned}
$$

Next, we compute the phase projection by numerical integration of (7.11), taking into 
account that $f$ is zero for $x<-W / 2$ and $x>W / 2$ :

$$
\begin{aligned}
F\left(y_{\mathrm{k}}, z_{\mathrm{k}}\right)=f_{0} d+\sum_{p=1}^{N}[ & A_{\mathrm{p}} \int_{-W / 2}^{W / 2} \sinh \left(\zeta_{\mathrm{p}} z_{\mathrm{k}} / a\right) J_{0}\left(\zeta_{\mathrm{p}} r_{\mathrm{k}} / a\right) d x \\
& \left.+B_{\mathrm{p}} \int_{-W / 2}^{W / 2} \cosh \left(\zeta_{\mathrm{p}} z_{\mathrm{k}} / a\right) J_{0}\left(\zeta_{\mathrm{p}} r_{\mathrm{k}} / a\right) d x\right] \\
& +\sum_{p=1}^{M}\left[C_{\mathrm{p}} \int_{-W / 2}^{W / 2} \sin \left(p \pi z_{\mathrm{k}} / c\right) I_{0}\left(p \pi r_{\mathrm{k}} / c\right) d x\right. \\
& \left.\quad D_{\mathrm{p}} \int_{-W / 2}^{W / 2} \cos \left(p \pi z_{\mathrm{k}} / c\right) I_{0}\left(p \pi r_{\mathrm{k}} / c\right) d x+f_{0} d\right] .
\end{aligned}
$$

The integration is carried out numerically for every point $(y, z)=\left(y_{\mathrm{k}}, z_{\mathrm{k}}\right)$, with $r_{\mathrm{k}}=$ $\sqrt{x^{2}+y_{\mathrm{k}}^{2}}$, corresponding with the measured $F_{\exp }\left(y_{\mathrm{k}}, z_{\mathrm{k}}\right)$. The parameters $A_{\mathrm{p}}, B_{\mathrm{p}}, C_{\mathrm{p}}$, $D_{\mathrm{p}}$ and $f_{0}$ are determined by a least squares fit to a measured projection $F_{\exp }\left(y_{\mathrm{k}}, z_{\mathrm{k}}\right)$ by means of linear regression. The reconstructed field then follows by substitution of the parameter values in (7.20). In our analysis we used $M=N=10$ to obtain high accuracy, without amplifying high-frequency noise Feature scaling is applied to improve the numerical stability of the linear regression. Since the integrals for the forward Abel transform only depend on the geometry of the problem, the method excels for large data sets with an identical coordinate system: The integrals have to be computed only once in that case and the inversion from any experimentally observed projection reduces to a few matrix multiplications to solve the regression problem.

\section{D Reynolds and Péclet number estimation in the vapour film}

The values of the Reynolds number, comparing the inertial and viscous effects in the vapour film, and of the Péclet number, comparing heat transfer by convection and conduction, are evaluated $a$ posteriori. It is reasonable to assess them above all in the neck region, where the vapour flow velocity attains its maximum [116]. The Reynolds number is then defined as $\operatorname{Re}=\left(\rho_{\mathrm{v}} U_{\text {neck }} h_{\text {neck }}^{2}\right) /\left(\mu_{\mathrm{v}} \ell_{\text {neck }}\right)$, where $U_{\text {neck }}$ is the velocity in the middle of the vapour film cross-section at the neck location, $h_{\text {neck }}$ is the neck thickness (cf. subsection 7.4.4) and $\ell_{\text {neck }}$ is the length of the neck region as measured at the height $2 h_{\text {neck }}$ of the vapour film profile. The maximum Re value for the parameters of the present study is thereby found to be of the order of 0.5 , justifying the dominant role of viscous forces in the vapor film assumed here (Stokes flow). Indeed, for an ethanol drop of $R=3.56 \mathrm{~mm}$ with $T_{s \Sigma}=330^{\circ} \mathrm{C}$, one can obtain $U_{\text {neck }}=1.75 \mathrm{~m} / \mathrm{s}$, $h_{\text {neck }}=58 \mu \mathrm{m}$ and $\ell_{\text {neck }}=906 \mu \mathrm{m}$ giving rise to the mentioned Re value. The Péclet number Pe $=\operatorname{PrRe}$ is then estimated to be of the order of 0.4 , as well, supporting $a$ posteriori the hypothesis of a mostly conductive heat transfer in (across) the vapour film. Here $\operatorname{Pr}=\nu_{\mathrm{v}} / \alpha_{\mathrm{v}} \sim 0.8$ is the Prandtl number, and $\nu_{\mathrm{v}}=\mu_{\mathrm{v}} / \rho_{\mathrm{v}}$ and $\alpha_{\mathrm{v}}$ are the kinematic viscosity and the thermal diffusivity of the vapour, respectively. 
(a)

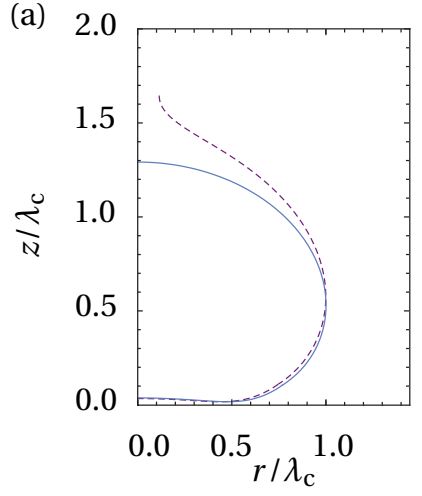

(c)

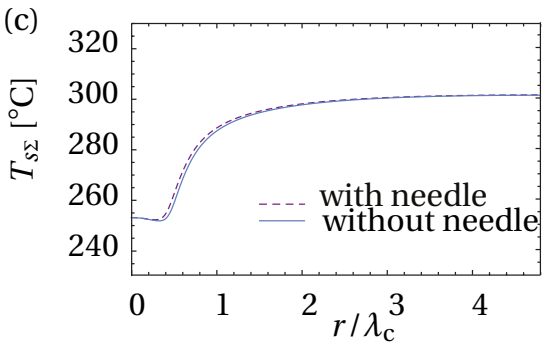

(b)
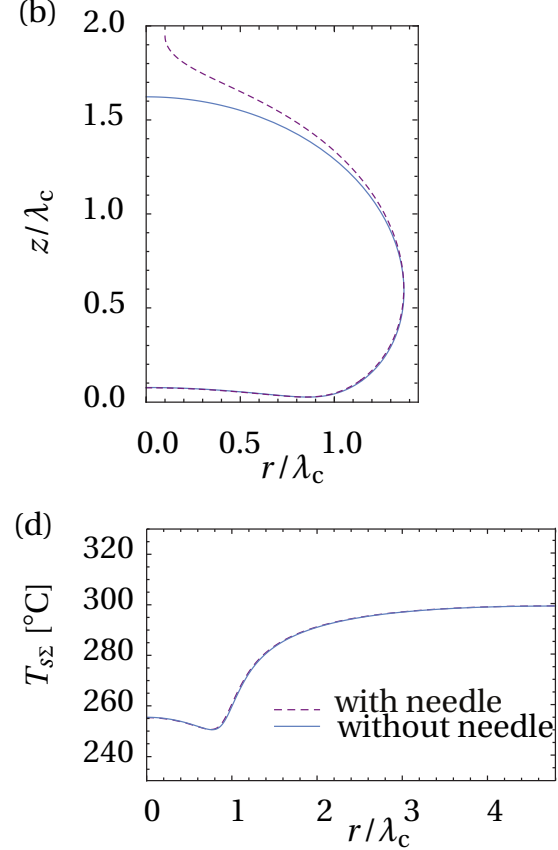

Figure 7.14: Numerically determined shape of an ethanol Leidenfrost drop with or without the presence of a needle (a-b), as well as the related temperature profiles at the plate surface (c-d) for $R=0.87 \lambda_{\mathrm{c}}$ and $R=1.37 \lambda_{\mathrm{c}}$, respectively, and $T_{\mathrm{imp}}=330^{\circ} \mathrm{C}$.

\section{E Influence of the needle}

While in the experiments the Leidenfrost drop is kept attached to a needle, the theory assumes a free Leidenfrost drop. In the present appendix, we numerically assess the actual influence of the needle on the Leidenfrost drop shape as well as on the temperature profile in the plate. Mathematically, incorporating the presence of a needle just consists in imposing as a boundary condition for the upper shape of the drop, governed by Equation 7.3, a vertical slope at the external needle radius (assuming complete wetting) rather than merely no singularity at the symmetry axis. Fig. 7.14 reports the results for an ethanol Leidenfrost drop over the quartz plate used in the experiments, as described in subsection 7.2.1, with an imposed temperature of $330^{\circ} \mathrm{C}$. As one can see, it is just the upper shape that is mostly affected by the presence of a needle. At the same time, the effect of the needle on the vapour film thickness and on the substrate temperature field is really minor, although it proves to be stronger for smaller drops as it could be expected. However, even for $R=0.87 \lambda_{\mathrm{c}}$, the smallest drop dealt with in the present setup, the neck thickness is affected only by $0.5 \%$, while the maximum substrate cooling only by $0.5 \mathrm{~K}$. Thus, we conclude that the presence of a needle is not really essential here. 


\section{Chapter 8}

\section{Theory for Leidenfrost drops on a liquid pool ${ }^{\dagger}$}

Droplets can be levitated by their own vapour when placed onto a heated plate, owing the to Leidenfrost effect. Here we study Leidenfrost drops levitated above a liquid pool, using an asymptotic analysis at small evaporation rates. The global shapes are found to resemble "superhydrophobic drops", that follow from a hydrostatic equilibrium. However, the morphology of the thin vapour layer between the drop and the pool is very different from the classical Leidenfrost drops, and exhibits different scaling laws. We determine analytical expressions for the vapour thickness as function of temperature and material properties, which are confirmed by numerical solutions. Finally, we show that deformability of the pool suppresses the chimney instability of Leidenfrost drops.

\footnotetext{
${ }^{\dagger}$ Michiel A.J. van Limbeek, Benjamin Sobac, Alexei Rednikov, Pierre Colinet and Jacco H. Snoeijer. To be submitted to Journal of Fluid Mechanics
} 


\subsection{Introduction}

A drop can be prevented from merging with a liquid bath when the bath is heated above the saturation temperature. Recently, such Leidenfrost drops have regained attention [111] after the first reports by Hickman [117], who more than half a century ago referred to these drops as "boules". Figure 8.1 gives an example of such a large water drop that is prevented from contacting a pool of water. The rapid evaporation induces thin vapour layer between the drop and the pool, and the corresponding vapour flow induces a pressure that keeps the drop separated from the pool.

Naturally, one tries to compare these "boules" to drops levitated above a heated plate. The latter have been studied in great detail $[4,15,17,18]$ since the first reports by Boerhaave and Leidenfrost $[5,7]$ centuries ago. Since then various studies have focussed on features as shape oscillations [22-26,28], drop mobility [118-120], dynamics during drop impacts $[8,36,45]$, and the Leidenfrost temperature [10,57].

Of particular interest is the shape of such Leidenfrost drops. When viewed from the side, a Leidenfrost drop above a plate resembles a sessile drop that makes a contact angle of $180^{\circ}$ with the substrate $[4,14-16]$. The vapour layer prevents a direct contact so that the droplet is maintained in a perfectly non-wetting, "superhydrophobic" state. Intriguingly, it is only fairly recently that the morphology of the thin vapour layer below the drop has been revealed. Experimentally, the shape was characterised by interferometry by Burton et al. [27], showing that the thickness of the vapour layer is not uniform. This is sketched in the left panel of Fig. 8.2, where one observes a large vapour pocket near center of the drop and a thin "neck" near its edge. For large drop radii, the base of the drop can even penetrate the top and the vapour escapes by a "chimney instability" [4,14]. Even prior to experiments, the details of the layer below levitated drops were predicted from a hydrodynamic analysis $[14,121,122]$, with a more complete description of the evaporation developed by Sobac et al. [16]. In the limit of small evaporation one indeed finds an asymptotically thin neck, and scaling laws for the thickness were established. One of the salient features is that the vapour pressure that carries the weight of the drop is nearly uniform below the drop: owing to the viscous vapour flow, the pressure falls abruptly across the thin neck to reach the atmospheric pressure.

Remarkably, Leidenfrost drops on a pool exhibit very different morphologies [111]. A typical numerical result is shown in the right panel of Fig. 8.2. Globally the drop can still be considered in a "superhydrophobic" state, but now on a deformable pool rather than on a substrate. The shape of the vapour layer, however, is very different from Leidenfrost drops on a substrate: the vapour layer is nearly uniform and exhibits oscillations before passing a thin neck. Also, the numerical analysis of [111] showed no indication of a chimney instability for Leidenfrost drops on a pool.

In this paper we investigate Leidenfrost drops on a pool based on a matched asymptotics analysis in the limit of small evaporation. We compute the detailed structure of the vapour layer as in Fig. 8.2, and establish the scaling laws for the thickness as a function of material properties and temperature difference. In section 8.2 we formulate the problem, sketch the asymptotic structure, and describe the numerical method used to validate our findings. Section 8.3 then focusses on the "boules" of Hickman [117], for which drops are levitated above a pool of the same liquid. Analytical results are obtained in the limit of large drops, explaining why, indeed, there is no chimney-instability 


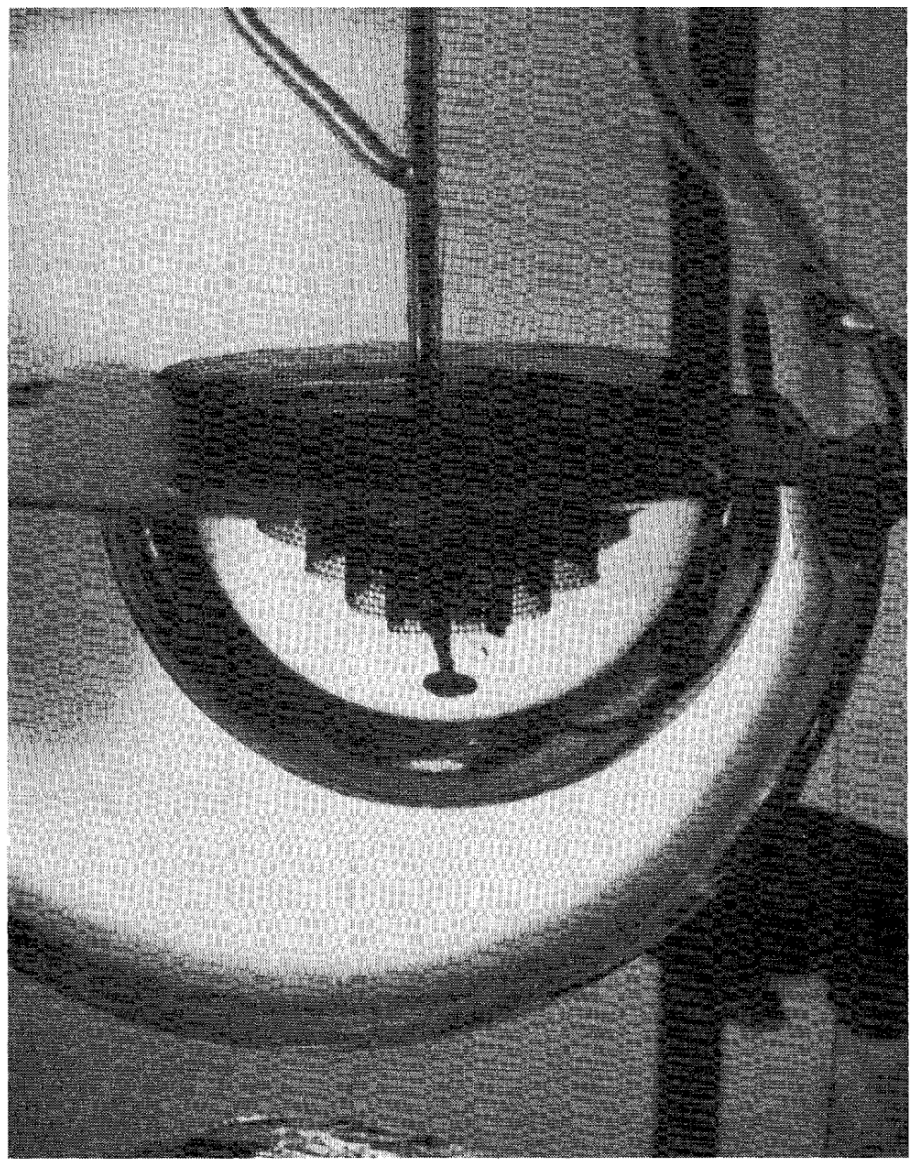

Figure 8.1: A Leidenfrost drop of water floating on a water bath that is heated a few degrees above the saturation temperature. The drop, also referred to as "boule" has a radius of $7 \mathrm{~cm}$, which is 25 times the capillary length. (Reprinted with permission from Hickman [117] 'Floating drops and liquid boules'. Copyright 2016 American Chemical Society.) 

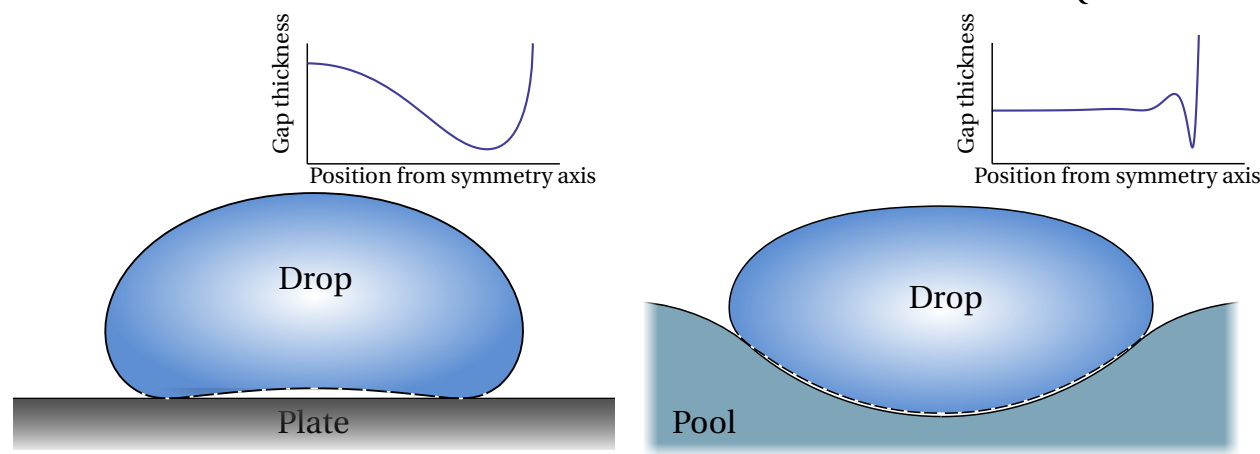

Figure 8.2: Sketches of a Leidenfrost drop levitated above a hot plate (left) and above a hot pool (right) based on numerical simulations. The resulting shapes are essentially "superhydrophobic drops" (solid lines), surrounded by a thin vapour layer (dashed lines). The insets provide a detailed zoom of the geometry of the vapour layer, revealing a striking difference between the two cases.

above a pool. The results are generalised in Section 8.4 for the case of smaller drops and differing liquids, showing that the scaling laws are robust. The paper closes with a conclusion in section 8.5.

\subsection{Formulation}

In this section we first present the equations that describe a steady Leidenfrost drop levitated above a liquid pool. This part follows the ideas presented in Maquet et al. [111], though the problem is formulated such that it is amenable to detailed analysis. We then sketch how the equations are solved by a matched asymptotic expansion. Also, we provide a brief description of numerical implementation that is used to validate analytical results.

\subsubsection{Model}

We first need to establish a convenient representation of the drop-on-pool geometry shown in the right panel of Fig. 8.2. The problem consists of two axisymmetric liquid domains, the drop and the pool, which we describe by the position of their respective liquid-vapour interfaces $h$ (for the drop) and $e$ (for the pool). These are defined in more detail in Fig. 8.3. While $h$ and $e$ in principle provide a full description of the geometry, we also introduce the thickness of the vapour layer $t$. This is convenient for describing the flow inside the thin vapour layer below the drop, where the two interfaces are essentially parallel.

Following previous theoretical developments on levitated drops [14, 16, 111, 122], we consider the liquid drop and pool to be at hydrostatic equilibrium while the flow of the produced vapour is treated in the lubrication limit. We first compute the vapour pressure $P_{\mathrm{v}}$ by approaching it from the side of the pool, 
2d outer solution: $\gamma, g$ $2 p$ outer solution: $\gamma, g$

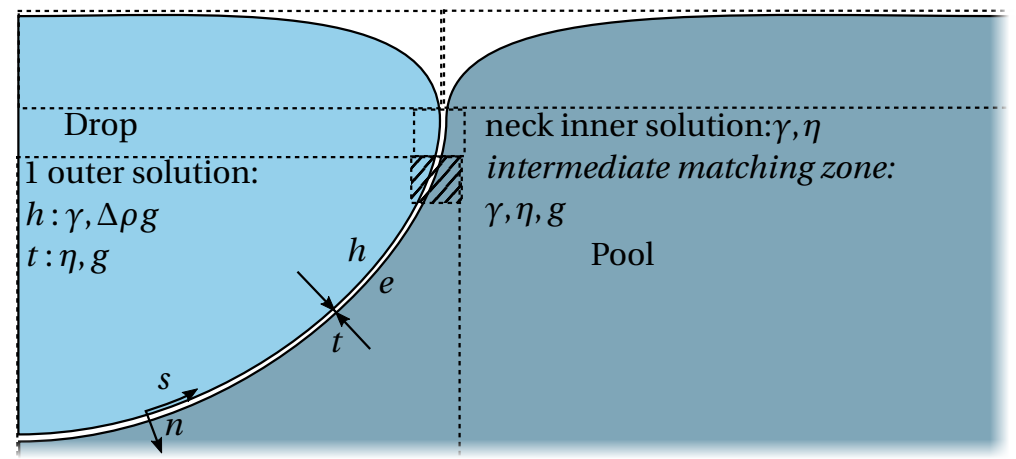

Figure 8.3: Sketch indicating different zones used for the asymptotic analysis for $\mathscr{E} \ll 1$, $\mathscr{R} \gg 1$ for identical fluid properties $(\Gamma=1, \mathrm{P}=1)$. The dominant force balances in the inner/outer regimes are indicated as capillary $(\gamma)$, viscous $(\eta)$, and gravitational ( $g$ ). Also defined are the vapour layer thickness $t$, which is the separation between the drop interface $h$ and the pool interface $e$, while $s$ and $n$ denote the orientation of the curvilinear coordinate system.

$$
P_{\mathrm{v}}=-\rho_{\mathrm{p}} g e+\gamma_{\mathrm{p}} \kappa_{\mathrm{e}} .
$$

Here we introduced the pool density $\rho_{\mathrm{p}}$ and surface tension $\gamma_{\mathrm{p}}$, while the $\kappa_{\mathrm{e}}$ is the curvature of the pool interface. The first term represents the hydrostatic pressure inside the pool, while curvature term gives the Laplace pressure jump due surface tension. Since the hydrostatic pressure inside the pool was taken $-\rho_{\mathrm{p}} g e$, the atmospheric pressure was set to zero. Similarly, we obtain an expression for the $P_{\mathrm{v}}$ from the side of the drop

$$
P_{\mathrm{v}}=k-\rho_{\mathrm{d}} g h-\gamma_{\mathrm{d}} \kappa_{\mathrm{h}},
$$

where $\rho_{\mathrm{d}}, \gamma_{\mathrm{d}}, \kappa_{\mathrm{h}}$ represent the droplet density, surface tension and curvature. In comparison to Equation 8.1, there is an extra constant $k$ due to the finite volume of the drop. For example we will find below that for very large drops $k \sim 1 / R$, where $R$ is the maximum droplet radius. Equations 8.1 and 8.2 give two separate expressions for $P_{\mathrm{v}}$, which in the lubrication approximation for thin layers must be identical. Therefore $(8.1,8.2)$ can also be seen as a relation between $h$ and $e$.

We now turn to the flow inside the vapour layer. It will be shown that for small evaporation rates the gap thickness $t$ is asymptotically small, justifying the use of the lubrication theory. We therefore consider the reduced Stokes equation for the parallel velocity $u$,

$$
\partial_{s} P_{\mathrm{v}}=\eta \partial_{n n} u,
$$

where $s$ is the curvilinear coordinate along the layer, while $n$ is the coordinate perpendicular to $h$ (see Fig. 8.3). Owing the small gas viscosity $\eta$, we can assume that no flow is induced inside the drop and the pool. As a consequence, we can solve Equation 8.3 with no-slip boundary conditions at $n=0$ and $n=t$, yielding a parabolic profile 


$$
u=6 \bar{u}\left(\frac{n}{t}-\frac{n^{2}}{t^{2}}\right)
$$

where introduced the thickness-averaged velocity

$$
\bar{u}=-\frac{t^{2} \partial_{s} P_{\mathrm{v}}}{12 \eta} .
$$

The lubrication problem is closed using the axisymmetric continuity equation,

$$
r \partial_{t}+\partial_{s}(r t \bar{u})=r \frac{\epsilon}{t}
$$

where $r$ is the distance from the axis of symmetry and $\partial_{t}$ is a time-derivative; in the remainder we will look for steady states so that time derivatives can be omitted. The source term on the right hand side of Equation 8.6 is due to the flux of vapour generated by evaporation, which modelled by Fourier's law can be expressed as [111]

$$
\epsilon=\frac{k_{\mathrm{v}} \Delta T}{L \rho_{\mathrm{v}}} .
$$

In this expression $k_{\mathrm{V}}$ and $\rho_{\mathrm{V}}$ respectively are the vapour thermal conductivity and density, $L$ the latent heat of evaporation and $\Delta T$ the temperature difference between the pool and the drop. The dimensions of $\epsilon$ are $\mathrm{m}^{2} / \mathrm{s}$, and thus represents the volumetric vapour flux exiting the gap per unit length.

In summary, Leidenfrost drops on a pool are described by:

$$
\begin{aligned}
-\frac{1}{12 \eta} \partial_{s}\left(r t^{3} \partial_{s} P_{\mathrm{v}}\right) & =r \frac{\epsilon}{t}, \\
P_{\mathrm{v}} & =k-\rho_{\mathrm{d}} g h-\gamma_{\mathrm{d}} \kappa_{\mathrm{h}}, \\
P_{\mathrm{v}} & =-\rho_{\mathrm{p}} g e+\gamma_{\mathrm{p}} \kappa_{\mathrm{e}},
\end{aligned}
$$

which are three coupled equations for the vapour pressure $P_{\mathrm{v}}$ and respectively for the droplet and pool interface profiles $h$ and $e$. These equations need to be complemented by geometric expressions for the interface curvatures $\kappa_{\mathrm{e}, \mathrm{h}}$ and the thickness of the vapour layer. Below the drop, the latter can be expressed as

$$
t(s)=\frac{h-e}{\left(1+\left(\partial_{s} h\right)^{2}\right)^{1 / 2}} .
$$

For the time being we will keep the equations in dimensional form. As will be explained below, the various regions indicated in Fig. 8.3 require different balances, each of which will call for different rescalings. The appropriate dimensionless forms will be introduced when solving for these different regions. In the end, the results are governed by four dimensionless parameters, namely

$$
\Gamma=\frac{\gamma_{\mathrm{p}}}{\gamma_{\mathrm{d}}}, \quad \mathrm{P}=\frac{\rho_{\mathrm{p}}}{\rho_{\mathrm{d}}}, \quad \mathscr{R}=\frac{R}{\lambda_{\mathrm{c}}}, \quad \mathscr{E}=\frac{6 \eta R \epsilon}{\rho_{\mathrm{d}} g \lambda_{\mathrm{c}}^{4}} .
$$


The first two are ratios of surface tension and density of the pool and the liquid. The third parameter $\mathscr{R}$ represents the size of the drop, here quantified by the maximum radius $R$ (implicitly varied by $k$ ) scaled by the capillary length $\lambda_{\mathrm{c}}=\left(\gamma_{\mathrm{d}} / \rho_{\mathrm{d}} g\right)^{1 / 2}$. Finally, the evaporation-induced viscous vapour flow is quantified by $\mathscr{E}$, made dimensionless in a combination that appears naturally in the lubrication flow.

\subsubsection{Structure of asymptotic analysis}

The analysis carried out in section 8.3 corresponds to the limit of small evaporation, $\mathscr{E} \ll 1$, for which the vapour thickness is expected to be asymptotically small. It turns out that the problem can be split into several regions, indicated schematically in Fig. 8.3. At large scales, there are two outer regions: 1 . below the drop, and 2. above the drop and pool. In the limit of vanishing $t$, these outer solutions will precisely correspond to "superhydrophobic drops" on a liquid pool. These are equilibrium solutions that can be computed from the static balance between surface tension and gravity.

Interestingly, the two outer solutions exhibit a mismatch in curvature, which implies a mismatch in the vapour pressure. As a consequence, the two outer regions must be connected by a small inner region where pressure gradients are important. In this "neck" region, of typical thickness $t_{\mathrm{n}}$, the viscous effects due to gas flow are balanced by surface tension (see Fig. 8.3). Matching the inner zone to the two outer solutions gives a closed problem that allows one to identify the relevant scaling laws. Most of our analysis will be carried out for drops of very large volume, such that the maximum radius $R$ is much larger than the capillary length $\lambda_{\text {c }}$. In this doubly asymptotic limit, $\mathscr{E} \ll 1$ and $\mathscr{R} \gg 1$, we anticipate and exploit the following hierarchy of length scales:

$$
t_{\mathrm{n}} \ll t_{0} \ll \lambda_{\mathrm{c}} \ll R
$$

Here $t_{0}$ is the typical film thickness below the drop outside the very thin neck region.

This structure resembles that of the Leidenfrost drop on a rigid solid substrate. However, the deformability of the pool brings in several intricate new features. First, it will turn out that the gap thickness below the drop $t_{0}$ emerges from a balance between gas viscosity and gravity. This is in stark contrast to the hydrostatic balance for normal Leidenfrost drops. Second, large $R$ drops do not even exist above on a rigid solid, since above a critical volume the droplets develop "chimneys". Hence, the deformability of the supporting phase allows for a completely new type of solution.

\subsubsection{Numerical solution}

To validate our analysis, we also present numerical solutions of the system (Equation 8.8, 8.9 and 8.10 ), providing the shapes of both the pool and the drop. The problem is solved in curvilinear coordinates to avoid numerical instabilities arising from the small slope approximation in a axisymmetric coordinate system. The solutions are to satisfy a zero slope at the symmetry axis, and a flat bath for the pool.

Since we expect the film thickness to approach zero thickness for vanishing flux, we also solve the problem without any vapour film. In this situation, the problem reduces to that of a "superhydrophic" drop, described by Equation 8.9 and 8.10, where $e=h$. Here we impose a interfacial tension between the drop and the pool $\gamma_{\mathrm{dp}}=\gamma_{\mathrm{p}}+\gamma_{\mathrm{d}}$, resulting 
in a contact angle of $180^{\circ}$. Once the droplet shape is known, an extension can be made by solving Equation 8.8 based on the shape and hydrostatic pressure obtained by the solution of the superhydrophic drop.

\subsection{A large drop on a pool of the same liquid}

Here we perform a detailed analysis for the "boules" reported by Hickman [117], shown in Fig. 8.1. In this case the pool and the bath are of the same liquid, and hence have equal density and surface tension, i.e. $\Gamma=\mathrm{P}=1$. The boules formed are much larger than the capillary length, $\mathscr{R} \gg 1$, and below we will consider the asymptotics for small evaporation, $\mathscr{E} \ll 1$.

\subsubsection{Outer region 1: below the drop}

\section{The droplet shape.}

The outer shape of the drop and pool can be obtained by combining (Equation 8.9) and 8.10):

$$
0=k-\rho g(h-e)-\gamma\left(\kappa_{\mathrm{h}}+\kappa_{\mathrm{e}}\right),
$$

where we used the identical material properties $\gamma=\gamma_{\mathrm{d}}=\gamma_{\mathrm{p}}$ and $\rho=\rho_{\mathrm{d}}=\rho_{\mathrm{p}}$. We anticipate the gap thickness, $t \sim h-e$, to be much smaller than the radius, in which case $\kappa_{\mathrm{h}} \simeq \kappa_{\mathrm{e}}$ and also the hydrostatic term can be neglected. Equation 8.14 then further simplifies,

$$
0=k-2 \gamma \kappa_{\mathrm{h}},
$$

imposing a shape of constant curvature. Below we will find that the matching condition requires the outer solution to be a perfect hemisphere. Hence, we can identify $k=2 \gamma / R$, where $R$ is the maximum radius of the drop.

\section{The vapour thickness.}

The spherical geometry of the outer solution, and thus of the vapour layer, suggests that the analysis of the lubrication flow will be most easily expressed using spherical coordinates (see Fig. 8.4a). In this coordinate system, the lubrication Equation 8.8 reads

$$
-\frac{1}{R \sin \theta} \partial_{\theta}\left[\sin \theta \frac{t^{3}}{12 \eta R} \partial_{\theta} P_{\mathrm{v}}\right]=\frac{\epsilon}{t} .
$$

This equation needs to be complemented by an equation for $P_{v}$, for example Equation 8.9, which in spherical coordinates simplifies to $P_{\mathrm{v}}=\rho g R \cos \theta-2 \gamma / R$. Importantly, away from the neck region around $\theta=\pi / 2$, the pressure is dominated by gravity owing to the hierarchy of scales $\lambda_{\mathrm{c}} \ll R$. This means that $P_{\mathrm{v}} \simeq \rho g R \cos \theta$, and the lubrication equation can then be expressed as 
(a)

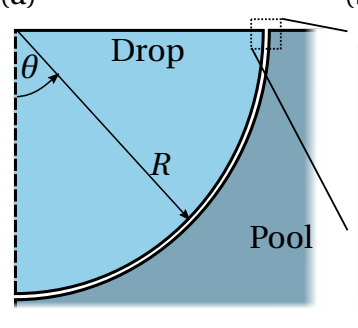

(b)

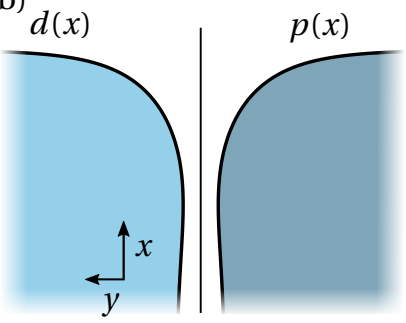

(c)

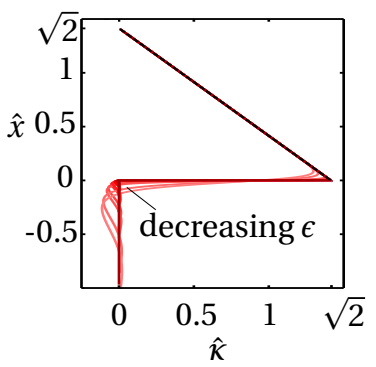

Figure 8.4: (a): Sketch of outer region 1. Since the shape below the drop reduces to a hemisphere, we adapt a spherical coordinate system where the gap thickness $t$ depends on the angle $\theta$. Panel (b) shows the local Cartesian coordinate system around $\theta=\pi / 2$, which is used for the analysis of the thin neck region at the exit of the vapour layer. The drop and pool interfaces are expressed as $d(x)$ and $p(x)$ respectively, while the vapour thickness reads $t=d-p$. (c): The interface curvature $\kappa$ in the neck region, obtained numerically from Equation 8.26 for various evaporation rates $\mathscr{E}$. A distinctive jump in curvature can be seen, indicating a sharp pressure jump across the thin neck. The dashed line shows the puddle solution for outer region 2 .

$$
-\frac{1}{t^{3} \sin \theta} \partial_{\theta}\left[\frac{1}{2} t^{3} \sin \theta \partial_{\theta}(\cos \theta)\right]=\frac{1}{t^{3} \sin \theta} \partial_{\theta}\left[\frac{1}{2} t^{3} \sin ^{2} \theta\right]=\frac{6 \eta \epsilon R}{\rho g t^{4}} .
$$

In this expression we collected all dimensional parameters on the right hand side, to form a dimensionless ratio.

The above expression invites us to introduce a dimensionless thickness $\tilde{t}=t / t_{0}$, where the characteristic scale for the thickness reads

$$
t_{0}=\left(\frac{6 \eta R \epsilon}{\rho g}\right)^{1 / 4}=\lambda_{\mathrm{c}} \mathscr{E}^{1 / 4} .
$$

Inserting this rescaling in Equation 8.17 and working out the derivatives gives

$$
\tilde{t}^{3} \partial_{\theta} \tilde{t}=\frac{2\left[1-\tilde{t}^{4} \cos \theta\right]}{3 \sin \theta},
$$

which is a first order ODE for the profile of the vapour layer $\tilde{t}(\theta)$. The solution to this equation can be cast in closed form

$$
\begin{aligned}
\tilde{t}^{4} & =\frac{8 \int_{0}^{\theta} d x(\sin x)^{5 / 3}}{3(\sin \theta)^{8 / 3}} \\
& =\frac{8}{3} \frac{1}{\sin \theta}\left(\frac{\pi^{\frac{1}{2}} \Gamma\left(\frac{1}{3}\right)}{5 \Gamma\left(\frac{5}{6}\right)}-\frac{1}{5} \cos \theta\left(2 \mathrm{H}_{2} \mathrm{~F}_{1}\left[\frac{1}{2}, \frac{2}{3}, \frac{3}{2},(\cos \theta)^{2}\right]+3(\sin \theta)^{\frac{2}{3}}\right)\right)
\end{aligned}
$$


where we imposed the boundary condition $\tilde{t}(0)^{\prime}=0$ to comply with the axisymmetric geometry. Here $\Gamma(x)$ is the Gamma function, $\mathrm{H}_{2} \mathrm{~F}_{1}$ is the Hypergeometric2F1 function and this expression was obtained using Mathematica. When plotting the solution (Equation 8.20) one observes that the vapour thickness is nearly constant. It very mildly increases from $\tilde{t}=1$ at $\theta=0$, to a slightly larger value at $\theta=\pi / 2$ (the exit of the gap):

$$
\tilde{t}_{\text {exit }}=2^{\frac{3}{4}} 15^{-\frac{1}{4}} \pi^{\frac{1}{8}}\left(\frac{\Gamma\left(\frac{1}{3}\right)}{\Gamma\left(\frac{5}{6}\right)}\right)^{\frac{1}{4}} \approx 1.22386 \cdots .
$$

\section{Summary and comparison to numerical solution.}

An important set of analytical results has thus been obtained. First, we found that the immersed part of the drop takes a hemispherical shape. Second, the vapour layer thickness at the axis below the drop is given by $\tilde{t}_{0}=1$. Third, the profile of the vapour layer in the outer zone is characterised by a single, universal profile, given by the expression (Equation 8.20). For the matching to the neck region (see the zoom of Fig. 8.4b), an important result is the thickness at the "gap exit" at $\theta=\pi / 2$, for which the analytical expression (Equation 8.21) is found. In dimensional form, we thus obtain the relevant thicknesses

$$
t_{0}=\lambda_{\mathrm{c}} \mathscr{E}^{1 / 4}, \quad t_{\text {exit }}=1.22386 \cdots \lambda_{\mathrm{c}} \mathscr{E}^{1 / 4} .
$$

These results were obtained under the assumption of the hierarchy of scales (Equation 8.13), which is self-consistent as long as

$$
\mathscr{E}^{1 / 4} \ll 1 \ll \mathscr{R} .
$$

To validate these findings, we compared the results to those obtained from the full numerical solution of Equation 8.8-8.10. Indeed, it is observed that for large drop volumes the immersed part of the droplet approaches a perfect hemisphere. In Fig. 8.5 we provide a further quantitative test, by plotting the central thickness $t_{0}$ for a droplet of radius $R=10 \lambda_{\mathrm{c}}$. Upon reducing the vapour generation parameter $\mathscr{E}$, the numerical result perfectly approaches the prediction (Equation 8.18). Moreover, evaluating $t_{0}$ for the experimental conditions of Fig. 8.1, we obtain $t_{0}=70 \mu \mathrm{m}$, which is of the same order as measured experimentally for an isopropanol boule: $25 \mu \mathrm{m}$ [123].

\subsubsection{Outer region 2: puddle solutions}

\section{Puddle solutions.}

We now turn to the second outer region, describing the top of the droplet and of the pool. In this range the vapour is no longer confined to a thin gap, so that viscous effects are completely negligible here. By consequence, the pool and drop are described by Equation 8.9 and 8.10 with $P_{\mathrm{v}}=0$. We further note that the constant $k \rightarrow 0$ for large drops, so that the profiles of the drop and the pool become each others mirror images. The solutions that result from these equations are the classical puddle solutions, for which the curvature increases linearly with depth. For explicit forms we refer to de Gennes et al. and Landau and Lifshitz [20, 124]. 


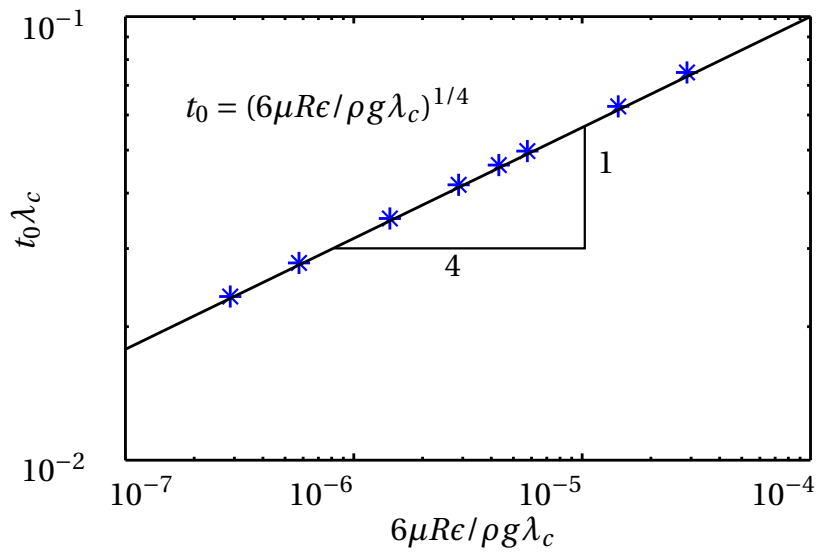

Figure 8.5: Numerical results of for the vapour thickness $t(0)$ by varying the vapour generation $\epsilon$. Results for a drop of $R=10 \lambda_{\mathrm{c}}$ on a pool having the same properties as the drop. The data points approach the black curve $t_{0}=(6 \eta R \epsilon / \rho g)^{1 / 4}$ for vanishing flux.

\section{Mismatch with outer solution 1.}

Importantly, the puddle solution exhibit a finite curvature at the droplet's edge, namely $\kappa=2^{1 / 2} / \lambda_{\mathrm{c}}$. This value of the curvature is obtained since, by symmetry, the puddle approaches the neck region vertically. This is to be contrasted with the curvature in the other outer region, below the drop, for which the curvature was found $1 / R$. For large drops, we thus find a "mismatch" in curvature near the exit of the vapour layer. This implies that the problem requires and inner zone that smoothly joins the two outer regions. The matching is illustrated in Fig. 8.4c, where we present a numerical solution to the problem (details are given in the paragraphs below). Panel b provides a detailed view of the droplet and pool profiles, and reveal a thin neck that is connected to the outer vapour layer. The right panel shows the corresponding curvatures, for various values of $\mathscr{E}$. The dashed line corresponds to the puddle solution; the numerical profiles smoothly join the puddle to the vapour film of vanishing curvature.

\subsubsection{Inner region: the neck profile}

\section{Matching conditions and numerical solution}

By inspection of Fig. 8.4, the thin neck region represents a small vertical zone around $\theta=\pi / 2$. We therefore adopt a local Cartesian coordinate system, as sketched in Fig. 8.4b, where gravity acts along the $x$-axis. In this coordinate frame, we describe the drop interface as $d(x)$ and the pool interface as $p(x)$. The gap thickness is then expressed as $t(x)=d(x)-p(x)$ and its curvature $\kappa_{\mathrm{t}}(x)=\kappa_{\mathrm{d}}(x)-\kappa_{\mathrm{p}}(x)$. The expressions for the pressure in the film from Equation 8.9 and 8.10), for equal liquid properties, can be written as 


$$
\begin{gathered}
P_{\mathrm{V}}=-\rho g x-\gamma \kappa_{\mathrm{d}} \\
P_{\mathrm{V}}=-\rho g x+\gamma \kappa_{\mathrm{p}} .
\end{gathered}
$$

Taking the difference of these equations, one finds $\kappa_{\mathrm{d}}=-\kappa_{\mathrm{p}}=\frac{1}{2} \kappa_{\mathrm{t}}$, resulting in a symmetric deformation for both $d$ and $p$. Equation 8.8 then becomes

$$
\frac{1}{12 \eta} \partial_{x}\left[t^{3} \partial_{x}\left[\operatorname{R\rho g} x+\frac{1}{2} \gamma \partial_{x x} t\right]\right]=\frac{\epsilon}{t} \approx 0,
$$

where we used the small slope representation of the curvature $\kappa_{\mathrm{t}}=t^{\prime \prime}$ for consistency with the lubrication approximation. The primes indicate spatial derivatives. Since we expect the local vapour generation in the small inner region to be negligible to the total generated flux, we can also drop the right hand side. Ultimately the gap profile needs to be matched to that of the puddle shape $d(x)$. It is therefore convenient to express the lubrication profile by $d(x)=t(x) / 2$, and non-dimensionalize all lengths by $\lambda_{\mathrm{c}}$ :

$$
\hat{d}=\frac{d}{\lambda_{\mathrm{c}}}=\frac{t}{2 \lambda_{\mathrm{c}}}, \quad \hat{x}=\frac{x}{\lambda_{\mathrm{c}}} .
$$

With this, we obtain after integrating Equation 8.24:

$$
\hat{d}^{\prime \prime \prime}=\frac{c^{3}}{\hat{d}^{3}}-1,
$$

where $c$ is an integration constant. This equation describes the shape of the drop interface inside the neck region.

The problem is closed by the matching conditions to the outer region 1 and 2, respectively corresponding to negative and positive limits of $\hat{x}$. The curvature of outer region 1 scales as $1 / R$, which in dimensionless variables give $\hat{d}^{\prime \prime} \sim 1 / \mathscr{R}$ and is thus asymptotically small for large drops. Therefore, we require $\hat{d}(-\infty)$ approaches a constant value, that according to Equation 8.26 can be equated to $c$. This thickness must ultimately match the "exit" thickness of outer zone 1, leading to the condition

$$
\frac{t_{\text {exit }}}{2}=d(-\infty) \quad \Longrightarrow \quad c=\frac{1}{2} \cdot 1.22386 \cdots \mathscr{E}^{1 / 4} .
$$

The boundary condition for positive $\hat{x}$ comes from matching the curvature of the puddle solution, which in scaled units reads $\hat{d}^{\prime \prime}=\sqrt{2}$ (see subsection 8.3.2). We numerically solved Equation 8.26 subject to these boundary conditions.

The resulting neck profiles are presented Fig. 8.6 for different values of $\mathscr{E}$, corresponding to different gap thickness according to Equation 8.27. One can observe that reducing the flux $\mathscr{E}$ leads to a localisation of the neck region, both in terms of thickness and lateral extent. To highlight these trends we have reported the profiles on two panels of double logarithmic scales, centred around the minimal neck thickness (see caption for details). The same data were used to show the mismatch in curvature in Fig. 8.4c. It is interesting to note that the profiles are strongly reminiscent to dimple profiles observed in dip-coating [125], which are indeed governed by an equation similar to Equation 8.26. Apart from the dimple, the dip-coating solutions also exhibit the oscillations seen in the left panel of Fig. 8.6, which were analysed in detail by Benilov et al. [126]. 

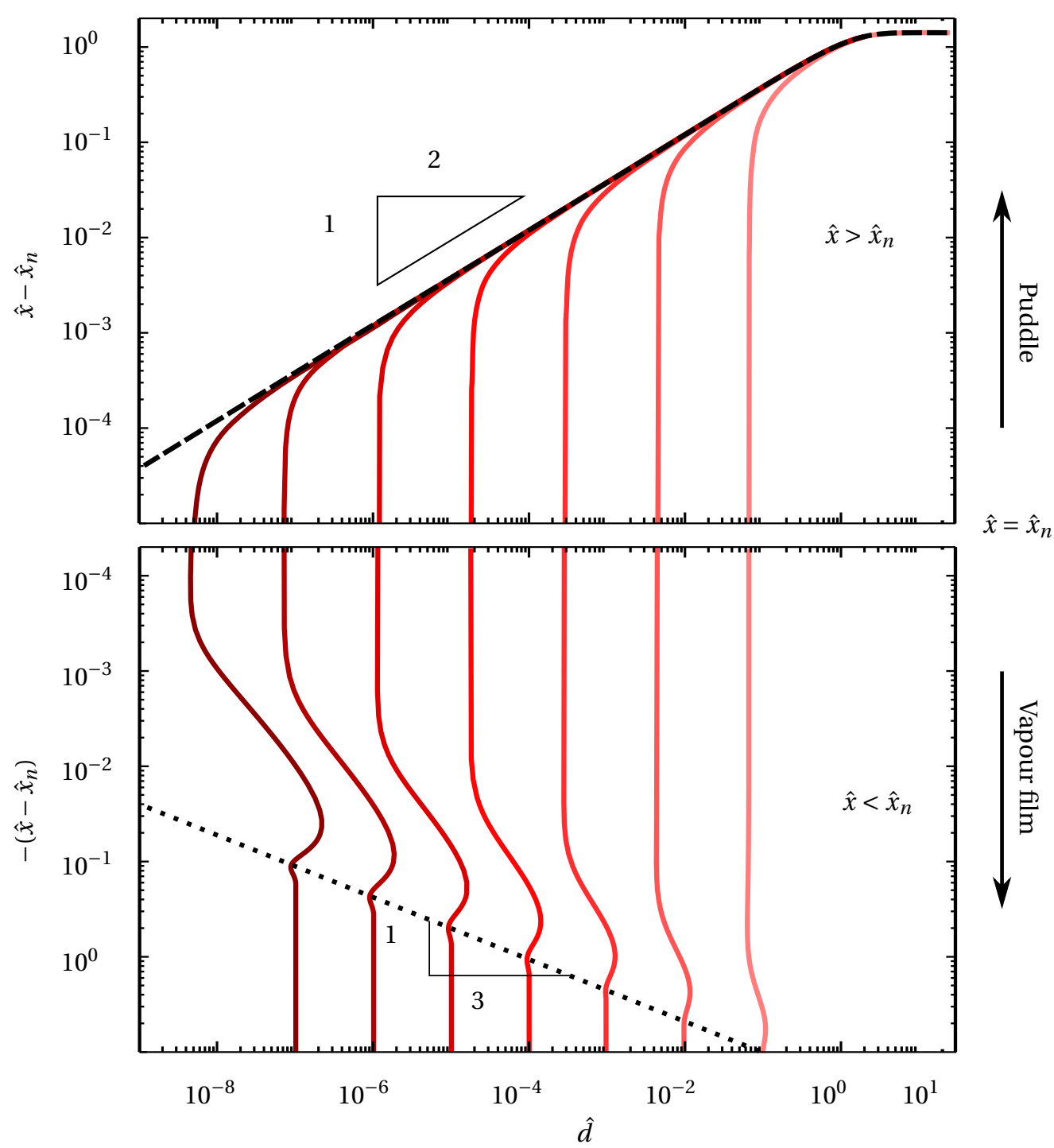

Figure 8.6: Neck profiles obtained from numerical solution of (8.26) for various vapour layer thicknesses $\hat{d}(-\infty) \sim \mathscr{E}^{1 / 4}$. The double logarithmic representation on the two panels, with inverse log-scale centered around $\hat{x}=\hat{x}_{\mathrm{n}}$, reveals the details of the thin neck region and the oscillations upon approaching the vapour film. The dotted line indicates the location of the oscillations, scaling as $\hat{d} \sim\left(\hat{x}_{\mathrm{n}}-\hat{x}\right)^{3}$. The dashed line shows the puddle solution for outer region 2 , exhibiting a $\hat{d} \sim\left(\hat{x}-\hat{x}_{\mathrm{n}}\right)^{2}$ upon approaching the neck. 


\section{Self-similar solution for the neck region}

Based on our numerical results we observe that the neck region near position $\hat{x}_{\mathrm{n}}$ becomes increasingly localized for small $\mathscr{E}$, while the neck thickness $\hat{d}_{\mathrm{n}}$ is found to decrease. This is in direct analogy to the neck region for normal Leidenfrost drops, above a rigid surface $[14,16]$. Owing to the smallness of $\hat{d}$ inside the neck, Equation 8.26 reduces to

$$
\hat{d}^{3} \hat{d}^{\prime \prime \prime}=c^{3}
$$

which means that the gravity is subdominant with respect to viscosity and surface tension. Indeed, Equation 8.28 is identical to the neck equation studied by Snoeijer et al. [14], which admits similarity solutions

$$
\hat{d}(x)=c^{\alpha} T(\zeta), \text { with } \zeta=\frac{\hat{x}-\hat{x}_{\mathrm{n}}}{c^{\beta}} .
$$

Inserting this Ansatz in Equation 8.28 this gives

$$
T^{3} T^{\prime \prime \prime}=1 \text {, and } 4 \alpha-3 \beta=3 .
$$

The exponents $\alpha, \beta$ can be determined from a matching condition for $\zeta \gg 1$, for which the shape of the pool $\hat{d}(x) \simeq \sqrt{2}\left(\hat{x}-\hat{x}_{\mathrm{n}}\right)^{2}$ must be approached, regardless of the value of $c$. This implies for large $\zeta$,

$$
c^{\alpha} T(\zeta) \simeq \sqrt{2} c^{\alpha} \zeta^{2}=\sqrt{2} c^{\alpha-2 \beta}\left(\hat{x}-\hat{x}_{\mathrm{n}}\right)^{2},
$$

hence, $\alpha-2 \beta=0$. Combined with Equation 8.30, this gives the exponents $\alpha=6 / 5$ and $\beta=3 / 5$.

While for large positive $\zeta$ we can impose the asymptotic boundary condition $T^{\prime \prime} \simeq$ $\sqrt{2}$, we still need to provide the asymptotics for negative $\zeta$. In principle, this could be obtained from a detailed analysis of the oscillatory approach to the thin film, in the spirit of the work on dip-coating [126]. Here we argue that the approach of the film $\hat{d} \sim c$ involves all terms in (8.26), such that $\hat{d}^{\prime \prime \prime}$ must be of order unity. In terms of scaling implies $\hat{d} \sim\left(\hat{x}_{\mathrm{n}}-\hat{x}\right)^{3}$. Indeed, this gives a good estimation for the position of the first oscillations in the left panel of Fig. 8.6 (dotted line). For the purpose of our analysis, this scaling law implies $\hat{d}^{\prime \prime} \sim\left(\hat{x}_{\mathrm{n}}-\hat{x}\right) \sim c^{1 / 3}$, which is asymptotically small. In terms of the similarity Ansatz (Equation 8.29), this means $T^{\prime \prime}(-\infty) \sim c^{1 / 3} \rightarrow 0$.

In summary, we expect the neck to be governed by a similarity solution $T(\zeta)$, which can be computed from Equation 8.30 subject to boundary conditions $T^{\prime \prime}(-\infty)=0$ and $T(\infty)=\sqrt{2}$. The numerical solution is given in Fig. 8.7, represented as a dashed line. The other curves correspond to the profiles of Fig. 8.6, scaled according to Equation 8.29. We observe a collapse onto the similarity solution as the value of $c \sim \mathscr{E}^{1 / 4}$ is reduced. The full relation for the minimum neck thickness can now be found by determining the minimum of the similarity function, which we numerically find to be $T_{\mathrm{n}}=1.147 \cdots$. Hence, we find

$$
\hat{d}_{\mathrm{n}}=1.147 \cdots c^{6 / 5},
$$

which provides the minimum thickness at the neck, as confirmed in Fig. 8.7b. 
(a)

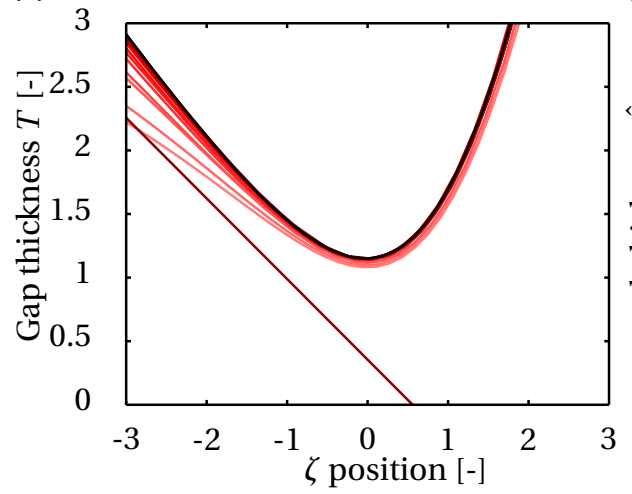

(b)

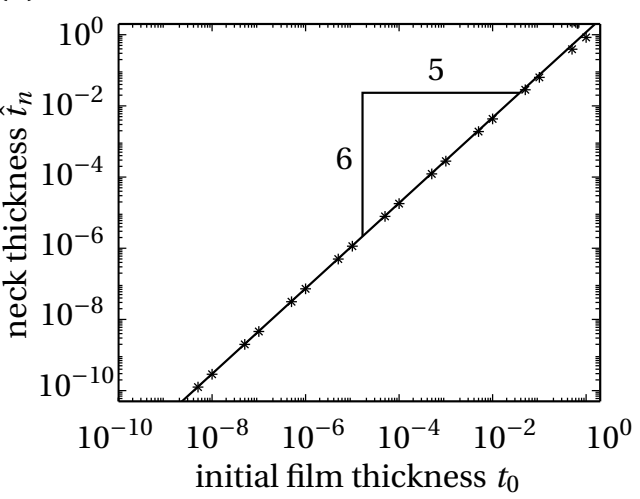

Figure 8.7: The neck region exhibits a self-similar structure, captured by the similarity function $T(\zeta)$ (dashed line). The other curves represent numerical profiles of the neck shown in Fig. 8.6, scaled according to $T=\hat{d} / c^{6 / 5}$ and $\zeta=\left(\hat{x}-\hat{x}_{\mathrm{n}}\right) / c^{3 / 5}$. The scaling is also found when solving Equation 8.8-8.10.

\subsubsection{Summary}

Let us now conclude the analysis for $\mathscr{E} \ll 1, \mathscr{R} \gg 1$ for the case the drop and the pool consist of the same liquid $(\Gamma=1, \mathrm{P}=1)$. We first recall the expressions for the vapour layer thickness below the center of the drop $\left(t_{0}\right)$ and the vapour thickness as it approaches the neck $\left(t_{\text {exit }}\right)$ :

$$
t_{0}=\lambda_{\mathrm{c}} \mathscr{E}^{1 / 4}, \quad t_{\text {exit }}=1.22386 \cdots \lambda_{\mathrm{c}} \mathscr{E}^{1 / 4} .
$$

These can now be complemented by the minimum thickness of the neck

$$
t_{\mathrm{n}}=2 \lambda_{\mathrm{c}} \hat{d}_{\mathrm{n}}=1.272 \cdots \lambda_{\mathrm{c}} \mathscr{E}^{3 / 10},
$$

which was obtained using Equation 8.27 and 8.32. The hierarchy of scales (Equation 8.13) is indeed satisfied as long as

$$
\mathscr{E}^{3 / 10} \ll \mathscr{E}^{1 / 4} \ll 1 \ll \mathscr{R}
$$

which completes the analysis.

\subsection{Finite drop sizes and differing liquids}

Until now we have studied the behaviour of infinitely large Leidenfrost drops on a liquid bath of equal physical properties. It is of course interesting to extend the results to smaller sized drops and to systems of different liquids. In the limit of small evaporation, $\mathscr{E} \ll 1$, one still finds that the vapour layer is asymptotically thin. Hence, the global shape of the drop is expected to be a "superhydrophobic" drop on a pool, governed by hydrostatics. Exploiting this idea, we demonstrate that the various scaling laws for the vapour layer are robust, as is confirmed by solving the full problem numerically. 


\subsubsection{Finite drop size}

Let us first focus on finite sized drops, while keeping $\Gamma=\mathrm{P}=1$. The size of the drop can be tuned by $k$ appearing in Equation 8.9, and a numerical example is presented in blue in Fig. 8.8. In this particular case the droplet radius $\mathscr{R}=3$; in general a relation $\mathscr{R}(k)$ can be established numerically. Comparing the droplet shape to the very large drops in Fig. 8.3, one finds that the immersed part of the drop still resembles a spherical cap, but the position of the neck has clearly shifted. The inset shows details of the vapour layer, which also has a similar structure as for large drops at small $\mathscr{E}$.

These features can be understood in detail. First, we compare the full numerical solution to the reduced (hydrostatic) calculation for the superhydrophobic drop, as described in subsection 8.2.3. The latter is shown as the red dashed curve in Fig. 8.8, indeed giving an excellent description of the global shape. As a second step, one can use this global shape to predict the gap thickness. Namely, the superhydrophobic drop provides $P_{\mathrm{v}}$ assuming a negligible influence of the lubrication flow in the vapour film; this is valid except for the thin neck region where viscous effects are important. Inserting this pressure profile in the lubrication Equation 8.8, one can obtain the vapour layer profile. The result is shown as the red dashed curve in the inset of Fig. 8.8, indeed giving an excellent quantitative agreement outside the oscillatory neck region.

How the scaling for the length scale $l_{\mathrm{n}}$ of the oscillations depends on $\mathscr{E}$ can be estimated using the previously obtained result $d \propto-\left(\hat{x}-\hat{x}_{\mathrm{n}}\right)^{3}$. This leads to $l_{\mathrm{n}} \propto \mathscr{E}^{1 / 12}$, where all prefactors are of order 1 , and for the further analysis of the outer zone of the gap to hold we obtain the restriction $\mathscr{R} \ll l_{\mathrm{n}}$.

Based on these observations we can now revisit the analysis for the vapour layer. For equal material properties and small evaporation, Equation 8.15 is still valid so that the immersed part of the drop has a constant curvature. For the numerical example in Fig. 8.8 we find $\lambda_{\mathrm{c}} \kappa_{\mathrm{h}}=0.29=1 / \mathscr{R}_{\mathrm{c}}$, where we define $\mathscr{R}_{\mathrm{c}}$ as the dimensionless radius of curvature of the spherical cap. Also, the lubrication Equation 8.19 is still valid. However, since the expression for $P_{\mathrm{v}}$ involves $\mathscr{R}_{\mathrm{c}}$ rather than $\mathscr{R}$, we need to adapt the expression for $t_{0}$ accordingly. With this Equation 8.18 simply becomes

$$
t_{0}=\lambda_{\mathrm{c}}\left(\frac{\mathscr{R}_{\mathrm{c}}}{\mathscr{R}}\right)^{1 / 4} \mathscr{E}^{1 / 4}
$$

where the ratio $\mathscr{R}_{\mathrm{c}} / \mathscr{R}$ can be inferred from the superhydrophobic drops. When computing the thickness of the very thin neck, one should also take into account that the neck is no longer positioned at $\theta=\pi / 2$ : in the example in Fig. 8.8 we find $\theta_{\mathrm{n}}=0.98$, which will lead to a small change in the thickness at the "exit" of the outer region, $t_{\text {exit }}$. Carrying through this minor modification, we find the neck thickness to be

$$
t_{\mathrm{n}}=2 \cdot 1.147\left(\frac{\tilde{t}\left(\theta_{\mathrm{n}}\right)}{2}\right)^{6 / 5} \lambda_{\mathrm{c}}\left(\frac{\mathscr{R}_{\mathrm{c}}}{\mathscr{R}}\right)^{3 / 10} \mathscr{E}^{3 / 10},
$$

where $\tilde{t}\left(\theta_{\mathrm{n}}\right)$ is known analytically, see (8.20). Note that the limit of very large drops is recovered by setting $\mathscr{R}_{\mathrm{c}}=\mathscr{R}$ and $\theta_{\mathrm{n}}=\pi / 2$.

Since two prefactors of Equation 8.37 vary strongly for $\mathscr{R} \approx 1$, it is expected that $\hat{t}_{\mathrm{n}} \sim$ $\mathscr{R}^{3 / 10}$ is only observed for large $\mathscr{R}$. This is in agreement with results from [111], where the results of the neck thickness were unfortunately identified to scale as $t_{\mathrm{n}} \propto \mathscr{R}^{1 / 4}$ : 


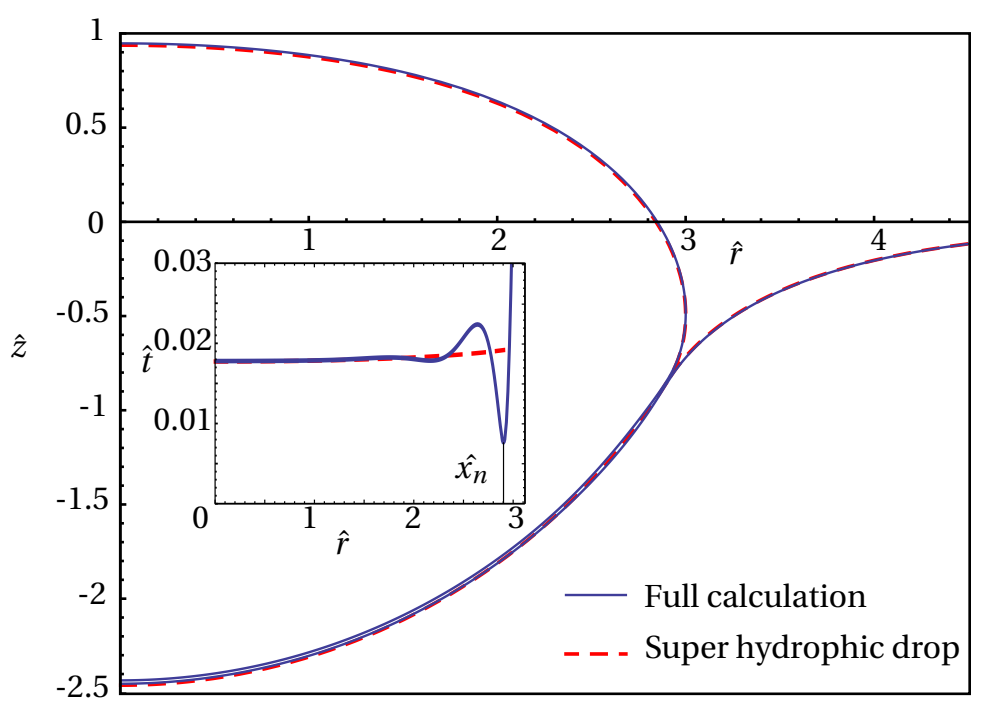

Figure 8.8: Profiles calculated for equal property liquids, where $\mathscr{E}=10^{-7}$. Both the super-hydrophobic drop calculation and the numerical simulation yield a spherical cap solution of curvature $\frac{1}{\mathscr{R}_{\mathrm{c}}}$ for the gap geometry. Note that $\mathscr{R}_{\mathrm{c}} \neq \mathscr{R}$ and the neck is positioned at $\theta_{\mathrm{n}}=0.98$.

The $\mathscr{R}$ dependence of the prefactors enshroud the limiting scaling for large $\mathscr{R}$ for the range of radii studied.

As we have seen, for the scalings of the outer zone to be properly shown, it is required that $\hat{l}_{\mathrm{n}} \ll \mathscr{R}$. As a consequence, since $t_{0} \sim \mathscr{E}^{1 / 12}$, in experimental conditions this condition is practically impossible to satisfy for $\mathscr{R} \approx 1$. Indeed, it is not surprising that the scaling laws for $t_{0}$ in the limit of small $\mathscr{R}$ [111] are strongly influenced by oscillations and only for $\mathscr{R}=4$ the scaling $t_{0} \propto \mathscr{E}^{1 / 4}$ is found. We therefore postulate a new hierarchy of lengthscales for which Equation 8.37 holds in the case of finite drop sizes.

$$
\begin{aligned}
t_{\mathrm{n}} & \ll t_{0} \ll l_{\mathrm{n}} \ll R, \quad \text { resulting in : } \\
\mathscr{E}^{3 / 10} & \ll \mathscr{E}^{1 / 4} \ll \mathscr{E}^{1 / 12} \ll \mathscr{R} .
\end{aligned}
$$

In summary, we conclude that the structure of the asymptotic analysis and the resulting scaling laws remain the same for finite sized drops, provided that the oscillations near the neck do not penetrate a large fraction of the gap length. Provided this is satisfied, in the case of equal liquid properties one can even compute the prefactors, provided that $\mathscr{R}_{\mathrm{c}}$ and $\theta$ are determined from the corresponding superhydrophobic drop. Note that the (dimensional) drop size $R$ appears both in $\mathscr{E}$ and in the prefactors of Equation 8.36 and 8.37. Hence, in terms of $R$ one observes a pure scaling relation only in the large drop limit. 


\subsubsection{Differing liquids}

We close the discussion by considering cases when $\Gamma=\gamma_{\mathrm{p}} / \gamma_{\mathrm{d}} \neq 1$ and $\mathrm{P}=\rho_{\mathrm{p}} / \rho_{\mathrm{d}} \neq 1$, i.e. when the drop and pool consist of a different liquid. This is for example the case in the experiments of ethanol drops on a silicone oil bath by Maquet et al. [111], who also provided a direct comparison between theory and experiment. Here we briefly represent typical numerical results of Fig. 8.9. The profiles in Fig. 8.9a are for varying $\mathrm{P}$ (keeping $\Gamma=1$ ), while $\Gamma$ is varied in $b$ (for fixed $P=1$ ).

At moderate values of the ratio of density (or surface tension), the droplets and vapour layer exhibit the expected morphological features: the vapour layer is of nearly uniform thickness below the drop, while the global shape is that of a superhydrophobic drop. Note that the immersed shape is no longer a spherical cap due to the difference in capillary length of the bath and droplet, due to different densities or surface tensions. A consequence of this is that we could no longer find a closed analytical expression for the vapour layer thickness. However, since outside the thin neck $P_{v}$ is of the order $\rho_{\mathrm{d}} g R$, the lubrication Equation 8.8 will still give rise to the scaling $t_{0} \sim \lambda_{\mathrm{c}} \mathscr{E}^{1 / 4}$. Consequently, the neck thickness scaling $t_{n} \sim \lambda_{\mathrm{c}} \mathscr{E}^{3 / 10}$ will also reappear.

In Fig. 8.9 we also consider the limit where the density or the surface tension of the bath is very large. While perhaps not physically relevant, the interest of this limit is that the bath becomes essentially undeformable: hence we should recover the Leidenfrost drop above a solid. Indeed, one observes that the drop is rising above the pool for increasing $\Gamma$ or $P$. Secondly, one observes the gap thickness at the symmetry axis increase as well. We thus find a gradual change from the "uniform thickness" that is typical for the pool, towards the characteristic "dimple" shape of Leidenfrost drops on a solid. This provides an explanation for why a chimney instability is suppressed by the bath's deformability. In addition, the morphological change has important consequences for the structure of the problem, since it changes the balances described in Fig. 8.3. This is the origin of the rather different scaling laws for the Leidenfrost drop on a pool and on a solid. 

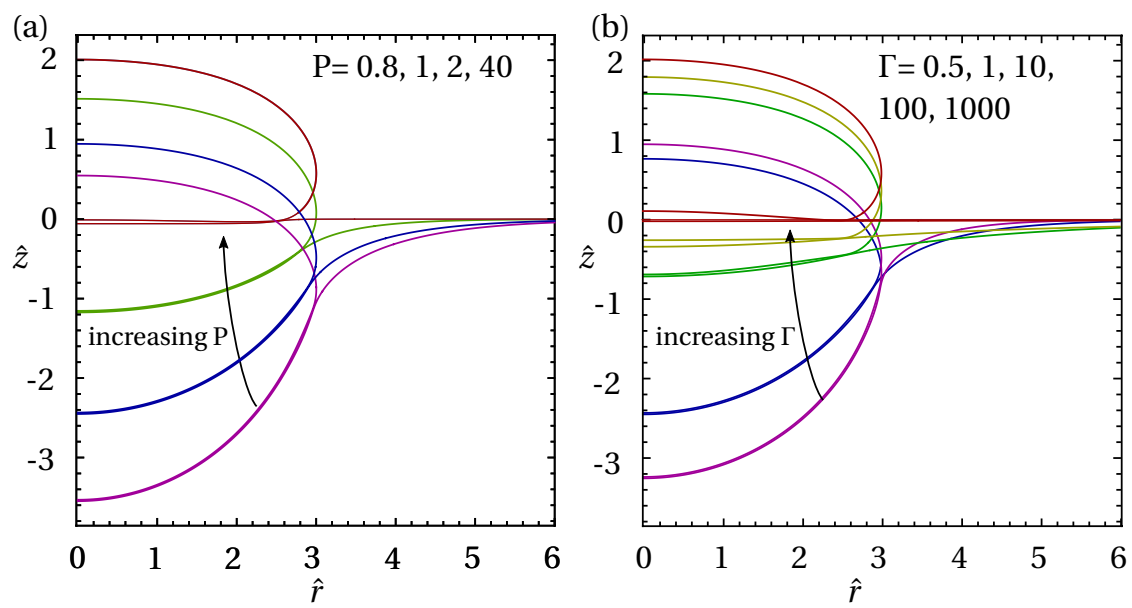

Figure 8.9: Numerical results for drops of radius $\mathscr{R}=3$ when varying the density (left) and the surface tension ratio (right) between the drop and the pool. The results indicate that in both situations, the limiting case of $P, \Gamma \rightarrow \infty$ the configuration resemble that of a Leidenfrost drop on a solid plate.

\subsection{Conclusion}

Leidenfrost drops on a superheated liquid bath were studied and their dynamics compared to that of Leidenfrost drops deposited on a superheated plate. Whereas the rigidity of the plate widens the vapour gap with increasing evaporation, the vapour gap here adjusts itself by submerging into the bath revealed. An outer zone is identified which only marginally increases in thickness with respect to the thickness at the lowest point, for which we found an analytic solution in the case of the liquid being of equal properties. We identified an inner zone, similar to drops on a plate, which is proceeded by stationary capillary waves. We developed scaling laws for both the closest point of the waves, called the neck, and the penetration depth of the waves into the outer region. A self-similar structure was found for the neck thickness, which showed excellent agreement with the full numerical solution of the problem. All these features depend strongly on the smallness of the evaporation number, which contains a mixture of both thermal and hydrodynamic properties of the system.

We tested the robustness of our analysis in the case of a small drop and differing liquids, and found that the limit of the evaporation number being zero, the system can be well described by a superhydrophobic drop on a liquid bath. Substitution of the pressure field obtained this way into the lubrication equation predicts the thickness of the vapour film very well, up to the oscillatory behaviour. Finally we found that the configuration of the plate can be recovered in the case of strongly differing liquid properties and hence a gradual change is expected between the two systems. 


\section{Conclusion and Outlook}

In this thesis we have discussed various aspects of drop impact on a hot surface, which at one hand is a paradigmatic system for the study of transient heat transfer phenomena, at the other hand also has its applications in the context of spray cooling. We have focussed on understanding how the heating of the impact target changes the touch-down and drop dynamics.

To study the touch-down and wetting dynamics of impacting drops we adopted frustrated total internal reflection (FTIR). In addition to the ability to discriminate between wetted and dry areas on the surface of the impact-target, the technique can measure the separation between the impacting drop and the plate within the evanescence-wave length scale. We have extended in chapter 2 the current model to convert the measured gray-values into a separation distance and showed that it is essential to take the polarisation of the light into account. The successful measurement of the air film between a lens and a plate showed good agreement with the analysis presented, proofing that modelling the film thickness as a simple decay of the measured intensity is too approximative.

\section{Impact dynamics on isothermal heated plates}

In chapter 3 we successfully employed the FTIR-technique to measure the separation of impacting drops on a superheated plate. We measured for the first time quantitatively the height of the droplet base while levitating on its on vapour film. We identified a new boiling regime in the velocity-temperature phase space, between contact and Leidenfrost boiling. This 'transition boiling regime' is characterized by the drop only partially touching the heated plate. At temperatures just below $T_{L}$ we have shown the existence of a circular ring within the evanescence length scale at the beginning of the impact process. This ring moves out radially with time, well described by Wagner's analogy. From this observation we obtain a crucial insight into the relevant length- and time scales, enabling future modelling of the dynamic Leidenfrost temperature.

In reduced ambient pressure conditions we found that the temperature range of the transition boiling regime changes: With decreasing ambient pressure, we observed that the lowest temperature for which the drop is in the transition regime decreases (see chapter 4). This observation correlates strongly with the static Leidenfrost temperature in the same reduced pressure condition and is at most weakly dependent of the impact velocity. The study provided strong indication that the two phenomena are the result of 
the same physical origin. Further study should indicate whether or not this behaviour is universal for all liquids and ambient gas pressures.

\section{Cooling effects during impact}

In the first chapters of the thesis we discussed the impacts on good thermal conducting plates. However, for some systems the touchdown is not dominated by the impact dynamics, but rather by thermal effects. In the later chapters the study of impacts on glass plates revealed that the plate cools down significantly as a result of the evaporation of the drop (chapter 5). Under those conditions, a drop can be initially in a Leidenfrost state. As a result of the low heat flux however, this situation can not be maintained and a delayed touch down is observed.

A secondary cooling mechanism was discovered in chapter 6: When impacting on thin, good thermal conducting plates, still cooling can be observed. In every impact scenario, the drawn from the plate results in the development of a thermal boundary layer. For impacting drops on good thermal conductors cooling effects can be observed when the thickness of the plate is of the order of the thermal boundary layer. In those situations, heat can only be supplied in the lateral direction of the plate, rather then from the 'infinite body', i.e. when the plate is much thicker than the boundary layer.

We developed a theory to estimate the thermal time scale of the heat transfer process which is strongly dependent on the plate thermal conductivity. Depending on the ratio between the impact time scale and the thermal time scale of the system, different behaviours can be observed: for good thermal conductors, the thermal time scale is much longer than the impact time scale and the plate is found to be isothermal during the impact process.

For poor thermal conductors, a direct way to study the cooling of the plate during impact can be achieved by operating the FTIR-system where FTIR is also obtained while the solid is in contact with the drop. The cooling is expected to lower the refractive index, thus changing the optical path length. When combining this with are reference beam, a fringe pattern is created from which the change in path length can be measured. The measurements will then give the (integrated) temperature change along the light path.

For systems where cooling is relevant, a spray can be observed (chapter 6). This spray is emitted from the top surface of the drop. The addition of FTIR-measurements, in addition to the commonly used side-view observations, revealed that entrapped bubbles remain on the top interface of the plate during the full duration of the impact process. For poor thermal conducting plates, the wetted area cannot be boiled away when the inertial forcing diminishes. When the drop flattens, these bubbles burst into the spray. We found that the spray is thus a signature of direct contact between the plate and the drop. For drops impacting an isothermal plate, the sprays can only be observed in the contact boiling regime. However, in the case of cooling effects, the sprays emerges the transition boiling regime as well.

In this thesis we focussed mainly on the properties of the plate. however, the way liquid properties affect the thermal time scale is still an open question. It is suspected that the enthalpy of evaporation might play a dominating role, especially for systems 
where the thermal time scale is of the order of the impact time scale.

\section{Cooling in solids exposed to evaporating static Leidenfrost drops}

The role of cooling was further investigated in the situation of the static Leidenfrost effect as well, see chapter 7 . We found that the finite conductivity of a quartz plate limits the heat transfer towards the evaporation front. As a result, less vapour is generated, resulting in a longer lifetime of the drop and a reduced vapour layer thickness. We proved both experimentally and numerically that the local temperature field under such a drop can be tens of Kelvin lower than expected and often reported from the set point temperature of the heater. The significance of the cooling is found to depend on the thermal conductivity ratio and thickness ratio of both the plate and the vapour layer. In experiments, the ratios are most easily altered by changing the properties of the plate.

\section{Leidenfrost drops on a deformable body}

A Leidenfrost drop on a deformable body was studied analytically in the context of a superheated pool of equal liquid properties (chapter 8). In this configuration, the balance between the gravitational, viscous and capillary forces changes completely. As a result, different scaling laws for the vapour thickness are found. Up to one numerical constant, we showed that the full system can be explored analytically and we obtained good agreement with existing literature. We found the existence of stationary capillary waves underneath the drop, which in many experimental conditions, can dominate the full geometry of the system. As a result, we conclude that no scaling law can be developed in those cases.

\section{A boiling curve for impacting drops?}

Finally it is interesting to a look again at the boiling curve introduced in chapter 1. Based on the studies described in this thesis, we can expect that the heat transfer rate of impacting drops drastically decreases when the drop is separated from the plate by the insulating vapour layer, which should therefore coincides with the onset of the transition boiling regime, reaching a local maximum when no touchdown occurs: the dynamic Leidenfrost temperature. The resulting boiling curve is sketched in Fig. 9.10, where the exact shape is expected to depend (partly) on the liquid and plate material properties, the drop size and impact velocity. We found that the onset of the transition boiling depends strongly on the ambient pressure and seems to correlate with the static Leidenfrost temperature. A more systematic study should investigate the universality of this observation for all liquids and ambient gas pressures. The dynamic Leidenfrost temperature was found to depend strongly on the impact velocity, as well as the thermal conduction of the plate. The relative localized and short duration of liquid-solid contact makes it difficult to determine the dynamic Leidenfrost temperature experimentally. However, from an application point of view the transition boiling regime is of much 


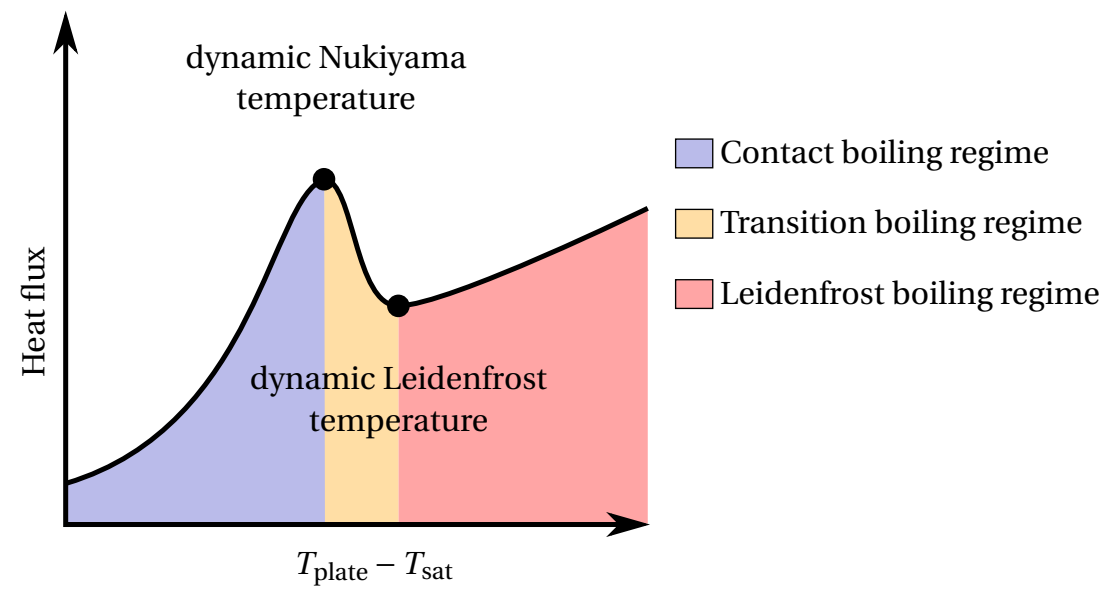

Figure 9.10: Boiling curve for impacting drops. Both the dynamic Nukiyama and Leidenfrost temperature are liquid-specific. The dynamic Nukiyama temperature is found to correlate with the static Leidenfrost temperature, decreasing with diminishing ambient gas pressure. The dynamic Leidenfrost temperature is elevated with increasing impact velocity and decreasing thermal conductivity properties. As a result the temperature range of the transition regime can vary a lot between different systems.

greater importance. Direct assessment of the heat transfer rate should give a conclusive outcome whether or adopting the boiling curve in the context of drop impact is valid and secondly, whether or not in addition to the dynamic Leidenfrost temperature, a dynamic Nukiyama temperature can be defined, coinciding with the onset of the temperature for the onset of the transition boiling regime. 


\section{Summary}

In this thesis we have discussed various aspects of drop impact on a hot surface, which at one hand is a paradigmatic system for the study of transient heat transfer phenomena, at the other hand also has its applications in the context of spray cooling. We have focussed on understanding how the heating of the impact target changes the touchdown mechanism and drop dynamics.

The spreading dynamics are strongly influenced by the solid-liquid interface. We therefore used for most of the studies frustrated total internal reflection (FTIR) as this method can discriminate between wetted and dry areas at this interface. The method also allows for a quantitative thickness measurement of thin air or vapour films up to a few hundreds of nanometre. We proposed and calibrated a new analysis model to improve the reliability of the method.

We applied the FTIR method for the study of the dynamic Leidenfrost effect, where a drop remains separated from a superheated plate during impact due to its own evaporation. We demonstrated the existence of a boiling regime, found in between the contact boiling and Leidenfrost boiling regime. This regime is referred to as transition boiling regime, in which a reduction of contact area is observed. At temperatures just below the dynamic Leidenfrost temperature, a circular ring of liquid-solid contact can be observed for a limited amount of time. This ring is a result of local high pressure peaks as a result of the decelerating drop, forcing the drop in contact with the plate. The outward spreading of said ring was found to be well described by theory for drop impact in the unheated case.

In reduced ambient pressure conditions we found that temperature range of the transition boiling regime changes: With decreasing ambient pressure, we observed that the lowest temperature for which the drop is in the transition regime decreases. This observation correlates strongly with the static Leidenfrost temperature in the same reduced pressure condition and is at most weakly dependent of the impact velocity. The study provided strong indication that the two phenomena are the result of the same physical origin.

In the first chapters of the thesis we discussed the impacts on good thermal conducting plates. In the later chapters we found that the plate cools down significantly as a result of the evaporation of the drop, a result of either the finite thickness of a thin plate or a low thermal conductivity of the plate material. In those conditions, a drop can be initially in a Leidenfrost state, but as a result of the cooling of the plate, the heat flux decreases and a delayed touch down is observed. We developed a theory to estimate the thermal time scale of the heat transfer process which is strongly dependent on the 
plate material.

For systems where cooling is relevant, a spray can be observed. This spray is emitted from the top surface of the drop. Our FTIR measurements, revealed that bubbles are entrapped remain there during the full impact process. When the drop flattens, these bubbles burst into the spray. The spray is thus a signature of direct contact between the plate and the drop and is observed in the contact boiling regime and the transition boiling as a result of cooling effects.

The role of cooling was also investigated in the situation of the static Leidenfrost effect. Here, a sessile drop is gently deposited on a superheated plate. We found that the finite conductivity of a quartz plate limits the heat transfer towards the evaporation front. As a result, less vapour is generated, resulting in a longer lifetime of the drop and a reduced vapour layer thickness. We have shown that taking the heater set-point as the Leidenfrost temperature can result in a large over-estimation. In the case of an ethanol drop on a glass substrate, the temperature difference between the two can be several tens of Kelvin. The significance of the cooling is found to depend on the thermal conductivity ratio and thickness ratio of both the plate and the vapour layer. In experiments, the ratios are most easily altered by changing the properties of the plate.

A Leidenfrost drop on a deformable body was studied analytically in the context of a superheated pool of equal liquid properties. In this configuration, the balance between the gravitational, viscous and capillary forces changes completely. As a result, different scaling laws for the vapour thickness are found. Op to one numerical constant, the full system can explored analytically and showed good agreement with existing literature. We found the existence of stationary capillary waves underneath the drop, which in many experimental conditions, can dominate the full geometry of the system. As a result, we conclude that no scaling law can be developed in those cases.

We have shown that when studying systems involving transient heat transfer phenomena it is of great importance to determine the relevant length and time-scales at play. Generally speaking one need to compare the duration of heat exchange between two bodies with the relevant thermal time scale of the system. When the thermal time scale is much longer, the system can be treated as isothermal, while in the other extreme, the process cannot remain isothermal and cooling effects dominate the process. The studies presented here shed new light on the influence of the plate temperature on the drop impact dynamics, leading to a better understanding of heat transfer processes in cooling applications. 


\section{Samenvatting}

In dit proefschrift worden verschillende aspecten bediscussiëerd omtrent inslaande druppels op hete oppervlaktes, hetgeen aan de ene kant een paradigmatisch system is om tijdsafhankelijke warmteoverdrachtsfenomenen te bestuderen, en aan de andere kant ook zijn toepassing heeft gevonden in de context van spray cooling. We focussen de aandacht op het doorgronden hoe de initiële temperatuur van een verhit oppervlakte het mechanisme van contact-maken en de druppel dynamica beïnvloed.

Het spreidingsmechanisme van de druppel na inslag wordt sterk beïnvloed door het vloeistof-plaat grensvlak. We gebruiken voornamelijk gefrustreerde totale interne reflectie (FTIR), omdat deze meetmethode verschil meet tussen natte en droge plekken op de plaat. Verder kan met FTIR de dikte van de damplaag tussen de druppel en de plaat meten tot een maximum afstand van een paar honderd nanometer. We stellen een nieuw analyse model voor en testen deze met kalibratiemetingen om de betrouwbaarheid van de methode te verbeteren.

Vervolgens passen we de FTIR methode toe in de studie naar het dynamische Leidenfrost effect, waarbij een druppel gescheiden blijft van een oververhitte plaat tijdens de inslag als gevolg van zijn eigen verdamping. We demonstreren het bestaan van een kook-regime, wat gevonden kan worden tussen het contact boiling regime en het Leidenfrost boiling regime. Dit nieuwe kook-regime wordt transition boiling regime gedoemd, wat gekarakteriseerd wordt doordat de druppel slechts gedeeltelijk contact maakt met de plaat. Wanneer de plaat temperatuur net onder de dynamische Leidenfrost temperatuur is kan tijdelijk een cirkelvormige ring van contact worden waargenomen. De ring is een gevolg van hoge drup pieken als gevolg van de afremmende druppel, wat resulteert in direct contact tussen de druppel en de plaat. De manier van hoe deze ring uitspreidt kan goed worden beschreven met modellen voor druppel inslag waar verhitting geen rol speelt.

Uit onze experimenten blijkt dat de omgevingsdruk een sterke invloed heeft op het temperatuursgebied waarin transition boiling gevonden kan worden. De dynamische Leidenfrost temperatuur veranderd slechts weinig wanneer de omgevingsdruk wordt verlaagd. Echter, met afnemende druk wordt een lagere begin temperatuur van dit regime gevonden. Dit gedrag heeft een sterke correlatie met de statische Leidenfrost temperatuur op deze druk. Dit suggereert dat de twee fenomenen vanuit eenzelfde fysisch mechanisme zijn te verklaren.

In de eerste hoofdstukken van het proefschrift hebben we ons beperkt tot studies van druppel inslagen op goede thermische geleiders. In de latere hoofdstukken beschrijven we dat de plaat sterk kan afkoelen tijdens de inslag als gevolg van de beperkte dikte van 
de plaat, ofwel de relatief lage warmteoverdrachtscoëfficiënt van het materiaal van de plaat. In deze gevallen kan de druppel in eerste instantie in een Leidenfrost staat zijn, maar, als een gevolg van de beperkte warmte stroom, op een later tijdstip de damplaag niet voldoende aangevuld kan worden en de druppel toch contact maakt. Met behulp van theorisch model kunnen we een karakteristieke thermische tijdsschaal bepalen van het systeem, welke sterk af hangt van de thermische eigenschappen van de plaat.

In het geval dat de koelingseffecten een rol spelen, kan vanaf de bovenkant van de druppel een spray aan druppeltjes worden waargenomen. Onze FTIR metingen laten zien dat bubbels die ingesloten zijn op het oppervlak van de plaat hier blijven gedurende het volledige inslagproces. Wanneer de bovenkant van de druppel vlakker en vlakker wordt door de inslag zullen deze bubbels hiermee versmelten. Dit resulteert in de spray die vanaf de bovenkant van de druppel komt en is dus een vingerafdruk voor het bestaan van direct contact tussen de plaat en de druppel en kan worden waargenomen in het contact- en transition boiling regime.

Het effect van koeling is ook bestudeerd in het geval van statische Leidenfrost druppels. Hier wordt een druppel voorzichtig bovenop een verhitte plaat gepositioneerd, waarop deze blijft zweven op zijn eigen damplaag. De eindige thermische geleidingscoëfficient van een kwarts plaat echter limiteert de warmte overdracht naar het verdampingsfront. Als een direct gevolg wordt minder damp gegenereerd, wat resulteert in een langere levensduur van de druppel en een dunnere damplaag. We laten zien dat het gebruik van de verre-veld temperatuur van het verhittingselement een verkeerde indicatie van de daadwerkelijke statische Leidenfrost temperatuur is, omdat dit effect wordt bepaald door het lokale temperatuurveld onder de druppel. In ons specifieke geval van een ethanol druppel op een kwartsplaat gaf dit een verschil van meerdere tientallen graden. De sterkte van de koeling hangt af van de ratio van de diktes en de ratio van de thermische geleiding van de plaat en de damplaag. Deze ratio's zijn experimenteel het eenvoudigste te veranderen door de afmetingen of het type materiaal van de plaat aan te passen.

Een Leidenfrost druppel op een vervormbare ondergrond is analytisch bestudeerd in de context van een druppel op een verhit bad. De limiet van grote druppels van gelijke vloeistof als het bad liet zien dat de balans tussen viskeuze krachten en die resulterend van zwaartekracht of oppervlaktespanning zorgen voor een totaal andere balans dan wanneer de ondergrond niet vervormbaar is. Een direct gevolg hiervan is dat de schalingswetten voor de dikte van de damplaag verandert. Op één numerieke constante na is het gehele systeem analytisch beschreven, hetgeen goede overeenkomsten vertoont met bestaande literatuur. Het bestaan van stationaire capillaire golven onder de druppel is beschreven, die in experimentele situatie de volledige geometrie van het systeem kunnen domineren. In deze gevallen moet we concluderen dat geen schalingswetten kunnen worden opgesteld.

De overkoepelende les die uit al deze studies kan worden getrokken is dat het cruciaal is om de relevante lengte- en tijdsschalen vast te stellen wanneer men tijdsafhankelijke warmte overdrachtsproblemen bestudeert. In het algemeen dient de tijdsduur van de warmteoverdracht te worden vergeleken met de kortste relevante thermische tijdsschaal beide lichamen. Wanneer deze langer zijn dan de tijdsduur van de warmteoverdracht kan het proces als isotherm worden gezien, terwijl in het andere extreme dit niet het geval is en het systeem sterk afkoelt gedurende het proces. 
De studie beschreven in dit proefschrift werpt nieuw licht op de invloed van de plaat temperatuur op de inslagdynamica van druppels, wat leidt tot een beter begrip van warmte overdracht in koeltoepassingen. 


\section{Acknowledgements}

One of the aspects of a PhD-position is to develop oneself into an independent researcher. It is therefore not surprising to find this statement in the university letter when you are formally allowed to defend your thesis. Reflecting on the four years I dedicated to study the rich phenomenon of the Leidenfrost effect, I have become aware that being independent is something different than being isolated. In fact, I am very thankful I had a great amount of people around me who taught me, supported me, criticised me and challenged me. Without them, the work I proudly presented in this thesis would be of a much lesser quality and I hereby would like to thank all of you.

Let me first start with showing my gratitude for what I learned from my supervisors, Detlef, Chao, Andera and Jacco. By your encouragement, criticism and insight in the complexities of the phenomena you kept me enthusiastic throughout the study. I greatly value your input during all the meetings we had.

Minori, I really enjoyed working with you, we made a great team in which we explored the rich dynamics of Leidenfrost drops.

In addition, I thank the rest of team-boiling: Pascal, Martin (KS), Kirsten, Olinka and Paul for the nice studies we did/do together and all the fun we had. My thanks go to Bas, Gert-Wim and Martin (B) (many experiments were made possible by their expertise), as well as the administrative support of Joanita. Finally I'd like to thank the whole Physics of Fluids-group for the friendly and thriving atmosphere.

My thanks also go to Pierre, Benjamin and Alexei from Univerité Libre de Bruxelles for the fruitful interaction, resulting in two chapters of this thesis. For financial aspects of this thesis I thank the support of MESA+ institute for nanotechnology and the Nederlandse Organisatie voor Wetenschappelijk Onderzoek: NWO.

Voor het afronden van een proefschrift is echter meer nodig dan goede expirimenten doen, fenomenen doorgronden en leren van de experts. Tijdens mijn tijd als promovendus zijn er veel mensen geweest die mij ondersteund hebben op andere manieren dan ik hierboven noemde. Als eerste dank ik Frieda die van mij houdt, mij op allerlei manieren ondersteunt en ik heb voorrecht heb om mee getrouwd te zijn. Zelfs als je mijn gradiënt-plotjes afkraakt houd ik van je. Ik bedank ook mijn ouders en Jeroen, mijn broer die mij al die tijd liefdevol omringt hebben. Verder ben ik dankbaar dat ik met Frieda een soort tweede gezin kreeg: Palidus en Mamahenriëtte, Gerhard, Jenneke, Marco en Rik, bedankt voor alles wat jullie mij geven. Ook bedank ik mijn vrienden 
(sommige van jullie ken ik langer wel dan niet), voor alle gezelligheid en de dingen die we doen en delen.

Ik heb het voorrecht om op het werk bijgestaan te worden in dingen die niet met onderzoek te maken hebben. In het bijzonder wil ik Mathijs, Minori, Pascal and Rianne noemen. Ook wil ik Detlef en Jacco bedanken voor hun steun wanneer ik even in zwaar weer kwam. Tenslotte dank ik Ivor, zonder zijn magic latex skills zou dit proefschrift van veel mindere kwaliteit zijn.

S. D. G. 


\section{About the author}

Michiel van Limbeek was born on the third of August, 1988, in Harderwijk. His interest for the natural sciences was obvious when was young: Here he built a ballista for a high-school project and played with integrated circuits to build a burglar alarm. After high-school he went to the far-east of the Netherlands in 2006, to Enschede, where Michiel first studied Advanced Technology at the University of Twente (UT). In Enschede he found out that among the natural sciences, physics was the most interesting and he enrolled in the Applied Physics master.

Based on his preferences, Michiel chose to study fluid mechanics, as he found voltage-current graphs 'boring', aligning optics 'annoying' and liked to 'get nice movies'. He therefore joined the Physics of Fluids group (PoF) of Detlef Lohse and studied superheated liquids with Henri Lhuissier. Among the many things that Henri taught Michiel, it was ironically that the best way to study flows without disturbing is to employ optical techniques. Michiel graduate in 2012 cum laude, whereafter he stayed with PoF for a PhD-position. He joined the lab of Andrea Prosperetti at Johns Hopkins University in 2013 for four months and helped organizing the Droplets 15 conference at the UT.

Michiel also has a great interest in music, playing the bass guitar and both classical and jazz piano. He likes to play real time strategy games and to finish large $\left(6 \mathrm{~m}^{2}\right)$ jigsaw puzzles. He lives currently with his beloved wife Frieda in Enschede, where he also attends a local church.

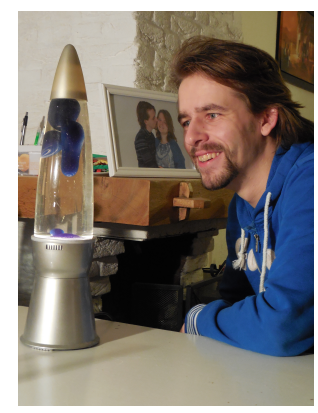

Figure 12.1: Michiel studying his two-phase Rayleigh-Bernard cell, dating from. 2002. The aspect ratio, based on the top diameter, $\Gamma=$ $3 / 10, \Delta T \approx 30 \mathrm{~K}$ and $\lambda_{c} \approx 5 \mathrm{~cm}$. The typical turnover time is roughly ten seconds. 


\section{Bibliography}

[1] A. Bejan, Heat Transfer. New York: John Wiley@ Sons, Inc., 1st ed., 1993.

[2] Z. Tamura and Y. Tanasawa, "Evaporation and combustion of a drop contacting with a hot surface," in Symposium (Int.) on Combustion, vol. 7, pp. 509-522, Elsevier, 1958.

[3] A. A. Mills and J. D. Fry, "Rate of evaporation of hydrocarbons from a hot surface: Nukiyama and Leidenfrost temperatures,” Eur. J. Physics, vol. 3, no. 3, p. 152, 1982.

[4] A.-L. Biance, C. Clanet, and D. Quéré, “Leidenfrost drops," Phys. Fluids, vol. 15, no. 6, pp. 1632-1637, 2003.

[5] H. Boerhaave, Elementa Chemiae. Lugdunum Batavorom, 1732.

[6] S. Nukiyama, "Maximum and minimum values of heat $q$ transmitted from metal to water under atmospheric pressure.," 1934.

[7] J. G. Leidenfrost, De aquae communis nonnullis qualitatibus tractatus. Duisburg: Ovenius, 1756.

[8] T. Tran, H. J. J. Staat, A. Prosperetti, C. Sun, and D. Lohse, "Drop Impact on Superheated Surfaces," Phys. Rev. Let., vol. 108, no. 3, p. 036101, 2012.

[9] G. K. Batchelor, An introduction to fluid dynamics. Cambridge university press, 2000.

[10] K. J. Baumeister and F. F. Simon, "Leidenfrost temperature - its correlation for liquid metals, cryogens, hydrocarbons, and water,” J. Heat Trans., vol. 95, no. 2, pp. 166-173, 1973.

[11] F. Demiray and J. Kim, "Microscale heat transfer measurements during pool boiling of fc-72: effect of subcooling," Int. J. Heat and Mass Trans., vol. 47, no. 14, pp. 3257-3268, 2004.

[12] N. Zuber, "Hydrodynamic aspects of boiling heat transfer (thesis)," tech. rep., California. Univ., Los Angeles; and Ramo-Wooldridge Corp., Los Angeles, 1959.

[13] J. Kim, "Spray cooling heat transfer: The state of the art," Int. J. Heat Fluid Flow, vol. 28, no. 4, pp. 753-767, 2007. 
[14] J. H. Snoeijer, P. Brunet, and J. Eggers, "Maximum size of drops levitated by an air cushion,” Phys. Rev. E, vol. 79, p. 036307, 2009.

[15] D. Quéré, “Leidenfrost Dynamics,” Ann. Rev. Fluid Mech., vol. 45, no. 1, pp. 197215, 2013.

[16] B. Sobac, A. Rednikov, S. Dorbolo, and P. Colinet, "Leidenfrost effect: Accurate drop shape modeling and refined scaling laws," Phys. Rev. E, vol. 90, no. 5, p. 053011, 2014.

[17] L. H. J. Wachters, H. Bonne, and H. J. van Nouhuis, "The heat transfer from a hot horizontal plate to sessile water drops in the spherodial state," Chem. Eng. Sci., vol. 21, pp. 923-936, 1966.

[18] J. D. Bernardin and I. Mudawar, "The Leidenfrost Point : Experimental Study and Assessment of Existing Models," J. Heat Trans., vol. 121, no. November, pp. 894 903, 1999.

[19] D. Arnaldo del Cerro, Á. G. Marílį̨, G. R. Römer, B. Pathiraj, D. Lohse, and A. J. Huis in't Veld, "Leidenfrost point reduction on micropatterned metallic surfaces," Langmuir, vol. 28, no. 42, pp. 15106-15110, 2012.

[20] P.-G. De Gennes, F. Brochard-Wyart, and D. Quéré, Capillarity and wetting phenomena: drops, bubbles, pearls, waves. Springer Science \& Business Media, 2013.

[21] Y. Pomeau, M. Le Berre, F. Celestini, and T. Frisch, “The Leidenfrost effect: From quasi-spherical droplets to puddles," Comptes Rendus Mécanique, vol. 340, no. 1112, pp. 867-881, 2012.

[22] N. J. Holter and W. R. Glasscock, "Vibrations of evaporating liquid drops," The Journal of the Acoustical Soc. of America, vol. 24, no. 6, 1952.

[23] R. Takaki and K. Adachi, "Vibration of a flattened drop. ii. normal mode analysis," J. Phys. Soc. of Japan, vol. 54, no. 7, pp. 2462-2469, 1985.

[24] D. E. Strier, A. A. Duarte, H. Ferrari, and G. B. Mindlin, "Nitrogen stars: morphogenesis of a liquid drop," Physica A: Statistical Mechanics and its Applications, vol. 283, no. 1-2, pp. $261-266,2000$.

[25] A. Snezhko, E. B. Jacob, and I. S. Aranson, "Pulsating-gliding transition in the dynamics of levitating liquid nitrogen droplets," New Journal of Physics, vol. 10, no. 4 , p. $043034,2008$.

[26] P. Brunet and J. H. Snoeijer, "Star-drops formed by periodic excitation and on an air cushion - a short review," Eur. Phys. J Special Topics, vol. 192, no. 1, pp. 207-226, 2011.

[27] J. C. Burton, a. L. Sharpe, R. C. A. van der Veen, A. Franco, and S. R. Nagel, "Geometry of the vapor layer under a Leidenfrost drop.," Phys. Rev. Let., vol. 109, no. 7, p. 074301, 2012. 
[28] W. Bouwhuis, K. G. Winkels, I. R. Peters, P. Brunet, D. van der Meer, and J. H. Snoeijer, "Oscillating and star-shaped drops levitated by an airflow," Phys. Rev. E, vol. 88, p. 023017, 2013.

[29] S. Thoroddsen, T. Etoh, and K. Takehara, "Air entrapment under an impacting drop," J. Fluid Mech., vol. 478, pp. 125-134, 2003.

[30] W. Bouwhuis, R. C. A. van der Veen, T. Tran, D. L. Keij, K. G. Winkels, I. R. Peters, D. van der Meer, C. Sun, J. H. Snoeijer, and D. Lohse, "Maximal Air Bubble Entrainment at Liquid-Drop Impact,” Phys. Rev. Lett., vol. 109, no. 26, p. 264501, 2012.

[31] J. de Ruiter, J. M. Oh, D. van den Ende, and F. Mugele, "Dynamics of collapse of air films in drop impact," Phys. Rev. Let., vol. 108, no. 7, p. 074505, 2012.

[32] H. Wagner, “Über Stoss- und Gleitvorgänge an der Oberfläche von Flüssigkeiten,” 1932.

[33] S. D. Howison, J. R. Ockendon, and S. K. Wilson, "Incompressible water-entry problems at small deadrise angles," J. Fluid Mech., vol. 222, pp. 215-230, 1991.

[34] R. Zhao and O. Faltinsen, "Water entry of two-dimensional bodies," J. Fluid Mech., vol. 246, pp. 593-612, 1993.

[35] J. Philippi, P.-Y. Lagrée, and A. Antkowiak, "Drop impact on a solid surface: shorttime self-similarity,” J. Fluid Mech., vol. 795, pp. 96-135, 2016.

[36] S. Chandra and C. T. Avedisian, "On the collision of a droplet with a solid surface," Proc. R. Soc. Lond. A: Math., Phys. Eng. Sci., vol. 432, no. 1884, pp. 13-41, 1991.

[37] I. V. Roisman, R. Rioboo, and C. Tropea, "Normal impact of a liquid drop on a dry surface: model for spreading and receding," in Proc. Royal Soc. London A: Math., Phys. and Eng. Sci., vol. 458, pp. 1411-1430, The Royal Society, 2002.

[38] C. Clanet, C. Béguin, D. Richard, and D. Quéré, "Maximal deformation of an impacting drop," J. Fluid Mech., vol. 517, pp. 199-208, 2004.

[39] J. Eggers, M. A. Fontelos, C. Josserand, and S. Zaleski, "Drop dynamics after impact on a solid wall: theory and simulations," Physics of Fluids (1994-present), vol. 22, no. 6, p. 062101, 2010.

[40] A. M. Worthington, "On the forms assumed by drops of liquids falling vertically on a horizontal plate," Proc. Royal Soc. London, vol. 25, no. 171-178, pp. 261-272, 1876.

[41] L. Xu, W. W. Zhang, and S. R. Nagel, "Drop splashing on a dry smooth surface," Phys. Rev. Let., vol. 94, no. 18, p. 184505, 2005.

[42] A. L. Yarin, "Drop impact dynamics: splashing, spreading, receding, bouncing...," Annu. Rev. Fluid Mech., vol. 38, pp. 159-192, 2006. 
[43] P. Tsai, R. C. A. van der Veen, M. van de Raa, and D. Lohse, "How micropatterns and air pressure affect splashing on surfaces," Langmuir, vol. 26, no. 20, pp. 1609016095, 2010.

[44] G. Riboux and J. M. Gordillo, "Experiments of Drops Impacting a Smooth Solid Surface: A Model of the Critical Impact Speed for Drop Splashing," Phys.Rev. Lett., vol. 113, no. 2, p. 024507, 2014.

[45] M. Shirota, M. A. J. van Limbeek, C. Sun, A. Prosperetti, and D. Lohse, "Dynamic Leidenfrost effect: relevant time and length scales," Phys. Rev. Let., vol. 116, no. 6, p. $064501,2016$.

[46] C. Cercignani, "Heat transfer in a Knudsen gas," Physica A: Stat. Mech. and its Appl., vol. 97, no. 2, pp. 440-454, 1979.

[47] V. K. Michalis, A. N. Kalarakis, E. D. Skouras, and V. N. Burganos, "Rarefaction effects on gas viscosity in the Knudsen transition regime," Microfluidics and nanofluidics, vol. 9, no. 4-5, pp. 847-853, 2010.

[48] H. Struchtrup, "Linear kinetic heat transfer: Moment equations, boundary conditions, and Knudsen layers," Physica A: Statistical Mechanics and its Applications, vol. 387, no. 8, pp. 1750-1766, 2008.

[49] J. Schilling, K. Sengupta, S. Goennenwein, A. Bausch, and E. Sackmann, “Absolute interfacial distance measurements by dual-wavelength reflection interference contrast microscopy," Phys. Rev. E, vol. 69, no. 2, p. 021901, 2004.

[50] T. Tran, H. J. J. Staat, A. Susarrey-Arce, T. C. Foertsch, A. van Houselt, H. J. G. E. Gardeniers, A. Prosperetti, D. Lohse, and C. Sun, "Droplet impact on superheated micro-structured surfaces," Soft Matter, vol. 9, no. 12, pp. 3272-3282, 2013.

[51] R. C. A. van der Veen, M. H. W. Hendrix, T. Tran, C. Sun, P. A. Tsai, and D. Lohse, "How microstructures affect air film dynamics prior to drop impact.," Soft Matter, vol. 10, no. 21, pp. 3703-7, 2014.

[52] E. Hecht, Optics. Boston: Addison Wesley Longman Inc, 4th ed., 1998.

[53] J. M. Kolinski, S. M. Rubinstein, S. Mandre, M. P. Brenner, D. A. Weitz, and L. Mahadevan, "Skating on a Film of Air: Drops Impacting on a Surface," Phys. Rev. Lett., vol. 108, no. 7, p. 074503, 2012.

[54] J. M. Kolinski, L. Mahadevan, and S. M. Rubinstein, "Lift-Off Instability During the Impact of a Drop on a Solid Surface," Phys. Rev. Let., vol. 112, p. 134501, 2014.

[55] J. M. Kolinski, L. Mahadevan, and S. M. Rubinstein, "Drops can bounce from perfectly hydrophilic surfaces,” Europhysics Let., vol. 108, p. 24001, 2014.

[56] M. Khavari, C. Sun, D. Lohse, and T. Tran, "Fingering patterns during droplet impact on heated surfaces," Soft Matter, vol. 11, pp. 3298-3303, 2015. 
[57] M. A. J. van Limbeek, M. Shirota, P. Sleutel, C. Sun, A. Prosperetti, and D. Lohse, "Vapour cooling of poorly conducting hot substrates increases the dynamic Leidenfrost temperature," Int. J. Heat and Mass Trans., vol. 97, pp. 101-109, 2016.

[58] C. Zettner and M. Yoda, "Particle velocity field measurements in a near-wall flow using evanescent wave illumination," Experiments in Fluids, vol. 34, no. 1, pp. 115-121, 2003.

[59] F. K. von Willisen et al., "Frustrated total internal reflection and application of its principle to laser cavity design,” Applied Optics, vol. 3, no. 6, pp. 719-726, 1964.

[60] S. Zhu, W. Yu, D. Hawley, and R. Roy, "Frustrated total internal reflection: A demonstration and review," Am. J. Physics, vol. 54, no. 7, pp. 601-607, 1986.

[61] U. D. Schwarz, "A generalized analytical model for the elastic deformation of an adhesive contact between a sphere and a flat surface," J. Colloid and Interface Science, vol. 261, no. 1, pp. 99-106, 2003.

[62] MATLAB, “version 8.2.0.701 (r2013b),"

[63] A. L. N. Moreira, A. S. Moita, and M. R. Panão, "Advances and challenges in explaining fuel spray impingement: How much of single droplet impact research is useful?," Progress in Energy and Combustion Science, vol. 36, no. 5, pp. 554 - 580, 2010 .

[64] D. Attinger, Z. Zhao, and D. Poulikakos, “An Experimental Study of Molten Microdroplet Surface Deposition and Solidification: Transient Behavior and Wetting Angle Dynamics,” J. Heat Trans., vol. 122, no. August, pp. 544-555, 2000.

[65] S. Mandre and M. P. Brenner, "The mechanism of a splash on a dry solid surface," J. Fluid Mech., vol. 690, pp. 148-172, 2012.

[66] G. E. Cossali, M. Marengo, and M. Santini, "Thermally induced secondary drop atomisation by single drop impact onto heated surfaces," Int. J. Heat and Fluid Flow, vol. 29, no. 1, pp. 167-177, 2008.

[67] H. Nair, H. J. J. Staat, T. Tran, A. van Houselt, A. Prosperetti, D. Lohse, and C. Sun, "The Leidenfrost temperature increase for impacting droplets on carbonnanofiber surfaces," Soft Matter, vol. 10, no. 13, pp. 2102-2109, 2014.

[68] V. Bertola, "An impact regime map for water drops impacting on heated surfaces," Int. J. Heat and Mass Trans., vol. 85, pp. 430-437, 2015.

[69] H. J. J. Staat, T. Tran, B. Geerdink, G. Riboux, C. Sun, J. M. Gordillo, and D. Lohse, Phase diagram for droplet impact on superheated surfaces, vol. 779. Cambridge Univ Press, 2015.

[70] X. Xu and T. Qian, "Hydrodynamics of Leidenfrost droplets in one-component fluids," Phys. Rev. E, vol. 87, p. 043013, 2013.

[71] M. Mani, S. Mandre, and M. P. Brenner, "Events before droplet splashing on a solid surface,” J. Fluid Mech., vol. 647, pp. 163-185, 2010. 
[72] Y. M. Qiao and S. Chandra, "Boiling of droplets on a hot surface in low gravity," Int. J. Heat and Mass Trans., vol. 39, no. 7, pp. 1379-1393, 1996.

[73] M. Shirota, M. A. J. van Limbeek, C. Sun, A. Prosperetti, and D. Lohse, "Dynamic Leidenfrost effect: Relevant time and length scales,” Phys. Rev. Lett., vol. 116, p. 064501, 2016.

[74] H. Lastakowski, F. Boyer, A.-L. Biance, C. Pirat, and C. Ybert, "Bridging local to global dynamics of drop impact onto solid substrates," J. Fluid Mech., vol. 747, pp. 103-118, 2014.

[75] L. H. J. Wachters and N. A. J. Westerling, "The heat transfer from a hot wall to impinging water drops in the spheroidal state," Chem. Eng. Sci., vol. 21, pp. 10471056, 1966.

[76] C. Josserand and S. T. Thoroddsen, "Drop impact on a solid surface," An. Rev. of Fluid Mech., vol. 48, pp. 365-391, 2016.

[77] L. Xu, "Liquid drop splashing on smooth, rough, and textured surfaces," Phys. Rev. E, vol. 75, p. 056316, 2007.

[78] L. Xu, L. Barcos, and S. R. Nagel, "Splashing of liquids: Interplay of surface roughness with surrounding gas,” Phys. Rev. E, vol. 76, p. 066311, 2007.

[79] L. Duchemin and C. Josserand, "Curvature singularity and film-skating during drop impact," Physics of Fluids, vol. 23, no. 9, 2011.

[80] S. Mandre, M. Mani, and M. P. Brenner, "Precursors to splashing of liquid droplets on a solid surface," Phys. Rev. Lett., vol. 102, no. 13, p. 134502, 2009.

[81] D. Orejon, K. Sefiane, and Y. Takata, "Effect of ambient pressure on Leidenfrost temperature,” Phys. Rev. E, vol. 90, no. 5, p. 053012, 2014.

[82] H. Kim, B. Truong, J. Buongiorno, and L.-W. Hu, "On the effect of surface roughness height, wettability, and nanoporosity on Leidenfrost phenomena," Appl. Phys. Lett., vol. 98, no. 8, p. 083121, 2011.

[83] H.-m. Kwon, J. C. Bird, and K. K. Varanasi, "Increasing Leidenfrost point using micro-nano hierarchical surface structures," Appl. Phys. Lett., vol. 103, no. 20, p. 201601, 2013.

[84] H. Fujimoto, Y. Oku, T. Ogihara, and H. Takuda, "Hydrodynamics and boiling phenomena of water droplets impinging on hot solid," Int. J. Multiphase Flow, vol. 36 , no. 8, pp. 620-642, 2010.

[85] B. T. Ng, Y. M. Hung, and M. K. Tan, "Suppression of the Leidenfrost effect via low frequency vibrations,” Soft Matter, vol. 11, pp. 775-784, 2015.

[86] A.-L. Biance, F. Chevy, C. Clanet, G. Lagubeau, and D. Quéré, "On the elasticity of an inertial liquid shock," J. Fluid Mech., vol. 554, no. -1, pp. 47-66, 2006. 
[87] E. Villermaux and B. Bossa, "Drop fragmentation on impact," J. Fluid Mech., vol. 668, pp. 412-435, 2011.

[88] R. B. Duffey and D. T. C. Porthouse, "The physics of rewetting in water reactor emergency core cooling,” Nucl. Eng. Des., vol. 25, no. 3, pp. 379-394, 1973.

[89] M. K. Agrawal and S. K. Sahu, "Analysis of conduction-controlled rewetting of a hot surface by variational method," Heat and Mass Trans., vol. 49, no. 7, pp. 963-971, 2013.

[90] V. K. Dhir, "Mechanistic prediction of nucleate boiling heat transfer-achievable or a hopeless task?," J. Heat Trans., vol. 128, no. 1, pp. 1-12, 2006.

[91] R. Verzicco, "Effects of nonperfect thermal sources in turbulent thermal convection," Phys. Fluids, vol. 16, no. 6, pp. 1965-1979, 2004.

[92] H. S. Carslaw and J. C. Jaeger, Conduction of heat in solids. Oxford: Clarendon Press, second ed., 1959.

[93] J. Philippi, P.-Y. Lagrée, and A. Antkowiak, "Drop impact on a solid surface : short time self-similarity,” arXiv:1504.05847v1, 2015.

[94] T. Toyoda and M. Yabe, "The temperature dependence of the refractive indices of fused silica and crystal quartz,” J. Physics D, vol. 16, no. 5, pp. L97-L100, 1983.

[95] G. S. Emmerson and C. W. Snoek, "The effect of pressure on the Leidenfrost point of discrete drops of water and freon on a brass surface," Int. J. Heat and Mass Trans., vol. 21, no. 8, pp. 1081-1086, 1978.

[96] J. D. Bernardin, C. J. Stebbins, and I. Mudawar, "Mapping of impact and heat transfer regimes of water drops impinging on a polished surface," Int.J. Heat Mass Trans., vol. 40, no. 2, pp. 247-267, 1997.

[97] H. Chaves, A. M. Kubitzek, and F. Obermeier, "Dynamic processes occurring during the spreading of thin liquid films produced by drop impact on hot walls," Int. J. Heatand fluid flow, vol. 20, no. 5, pp. 470-476, 1999.

[98] G. Emmerson, "The effect of pressure and surface material on the Leidenfrost point of discrete drops of water," Int. J. Heat and Mass Trans., vol. 18, no. 3, pp. 381-386, 1975.

[99] S. D. Aziz and S. Chandra, "Impact, recoil and splashing of molten metal droplets," Int. J Heat and Mass Trans., vol. 43, no. 16, pp. 2841-2857, 2000.

[100] L. Mach, “Über einen Interferenzrefraktor,” Zeitschrift für Instrumentenkunde, vol. 12, pp. 89-93, 1892.

[101] L. Zehnder, “Ein neuer Interferenzrefraktor," Zeitschrift für Instrumentenkunde, vol. 11, pp. 275-85, 1891.

[102] T. Kreis, Handbook of Holographic Interferometry. Wiley-VCH, 2005. 
[103] P. Plotkowski, Y. Y. Hung, and G. Gerhart, "Improved fringe carrier technique for unambiguous determination of holographically recorded displacements," Opt. Eng., vol. 24, no. 5, pp. 754-6, 1985.

[104] D. R. Matthys, J. A. Gilbert, T. D. Dudderar, and K. W. Koenig, "A windowing technique for the automated analysis of holo-interferograms," Optics and Lasers in Eng., vol. 8, pp. 123-136, 1988.

[105] J. Canny, "A computational approach to edge detection," IEEE Trans. Pattern Anal. Machine Intell., vol. 8, no. 6, pp. 679-698, 1986.

[106] M. A. Herráez, D. R. Burton, M. J. Lalor, and M. A. Gdeisat, "Fast two-dimensional phase-unwrapping algorithm based on sorting by reliability following a noncontinuous path.," Appl. Opt., vol. 41, no. 35, pp. 7437-44, 2002.

[107] I. H. Malitson, "Interspecimen comparison of the refractive index of fused silica," JOSA, vol. 55, no. 10, pp. 1205-9, 1965.

[108] P. E. Ciddor, "Refractive index of air: new equations for the visible and near infrared," Appl. Opt., vol. 35, no. 9, pp. 1566-73, 1996.

[109] V. Dribinski, A. Ossadtchi, V. A. Mandelshtam, and H. Reisler, "Reconstruction of Abel-transformable images: The Gaussian basis-set expansion Abel transform method," Rev. Sci. Instrum., vol. 73, no. 7, p. 2634, 2002.

[110] B. Sobac, P. Talbot, B. Haut, A. Rednikov, and P. Colinet, "A comprehensive analysis of the evaporation of a liquid spherical drop," J. Colloid Interface Sci., vol. 438, pp. 306-317, 2015.

[111] L. Maquet, B. Sobac, B. Darbois-Texier, A. Duchesne, M. Brandenbourger, A. Rednikov, P. Colinet, and S. Dorbolo, "Leidenfrost drops on a heated liquid pool," Phys. Rev. Fluids, vol. 1, p. 053902, 2016.

[112] M. Takeda, H. Ina, and S. Kobayashi, "Fourier-transform method of fringe-pattern analysis for computer-based topography and interferometry," JOSA, vol. 72, no. 1, p. 156, 1982.

[113] T. Kreis, "Digital holographic interference-phase measurement using the Fouriertransform method," JOSA A, vol. 3, no. 6, pp. 847-55, 1986.

[114] S. Ma, H. Gao, and L. Wu, "Modified Fourier-Hankel method based on analysis of errors in Abel inversion using Fourier transform techniques," Appl. Opt., vol. 47, no. 9, p. 1350, 2008.

[115] J. D. Jackson, "Boundary value problems in electrostatics II," in Classical Electrodynamics, ch. 3, New York: Wiley, 2 ed., 1998.

[116] D. Brutin, Droplet Wetting and Evaporation: From Pure to Complex Fluids. Academic Press, 2015.

[117] K. C. D. Hickman, "Floating drops and liquid boules," Industrial \& Eng. Chem.stry, vol. 56, no. 6, pp. 18-31, 1964. 
[118] H. Linke, B. J. Alemán, L. D. Melling, M. J. Taormina, M. J. Francis, C. C. DowHygelund, V. Narayanan, R. P. Taylor, and A. Stout, "Self-propelled Leidenfrost droplets,” Phys. Rev. Let., vol. 96, no. 15, p. 154502, 2006.

[119] A. Würger, "Leidenfrost gas ratchets driven by thermal creep," Phys. Rev. Let., vol. 107, no. 16, p. 164502, 2011.

[120] G. Lagubeau, M. Le Merrer, C. Clanet, and D. Quéré, "Leidenfrost on a ratchet," Nature Physics, vol. 7, no. 5, pp. 395-398, 2011.

[121] L. Duchemin, J. R. Lister, and U. Lange, "Static shapes of levitated viscous drops," J. Fluid Mech., vol. 533, pp. 161-170, 2005.

[122] J. R. Lister, A. B. Thompson, A. Perriot, and L. Duchemin, "Shape and stability of axisymmetric levitated viscous drops,” J. Fluid Mech., vol. 617, pp. 167-185, 2008.

[123] K. Hickman, J. Maa, A. Davidhazy, and O. Mady, "Features floating drops and liquid boules - a further look," Industrial \& Eng. Chem.stry, vol. 59, no. 10, pp. 1842, 1967.

[124] L. D. Landau and E. M. Lifshitz, "Fluid mechanics," Course of Theoretical Physics, vol. 6, 1959.

[125] J. H. Snoeijer, J. Ziegler, B. Andreotti, M. Fermigier, and J. Eggers, “Thick films of viscous fluid coating a plate withdrawn from a liquid reservoir," Phys. Rev. Let., vol. 100, no. 24, p. 244502, 2008.

[126] E. S. Benilov, S. J. Chapman, J. B. McLeod, J. R. Ockendon, and V. S. Zubkov, “On liquid films on an inclined plate," J. Fluid Mech., vol. 663, pp. 53-69, 2010. 
Temperature control is omnipresent in today's life: from keeping your fridge cold, maintaining a room at a pleasant temperature or preventing your computer from overheating. Efficient ways of heat transfer are often based on phase change, making use of the high latent heat of evaporation. In the context of spray cooling, liquid drops are impacting a hot plate to ensure a rapid cooling. At some temperature however, no contact occurs between the liquid and the plate, and the heat transfer rate is strongly reduced.

The origins of this so-called Leidenfrost phenomenon are investigated in this thesis from an experimental point. By investigating the wetting behaviour and bubble generation at the interface between a heated plate and a liquid drop, new light is shed on the onset of this undesirable phenomenon. The identification of the relevant time- and lengthscales give insight on how the both liquid an solid properties influence the onset of the Leidenfrost effect. These findings allow for a better prediction of the heat transfer between drops and a heated plate, aiding the optimalization of efficient cooling processes.
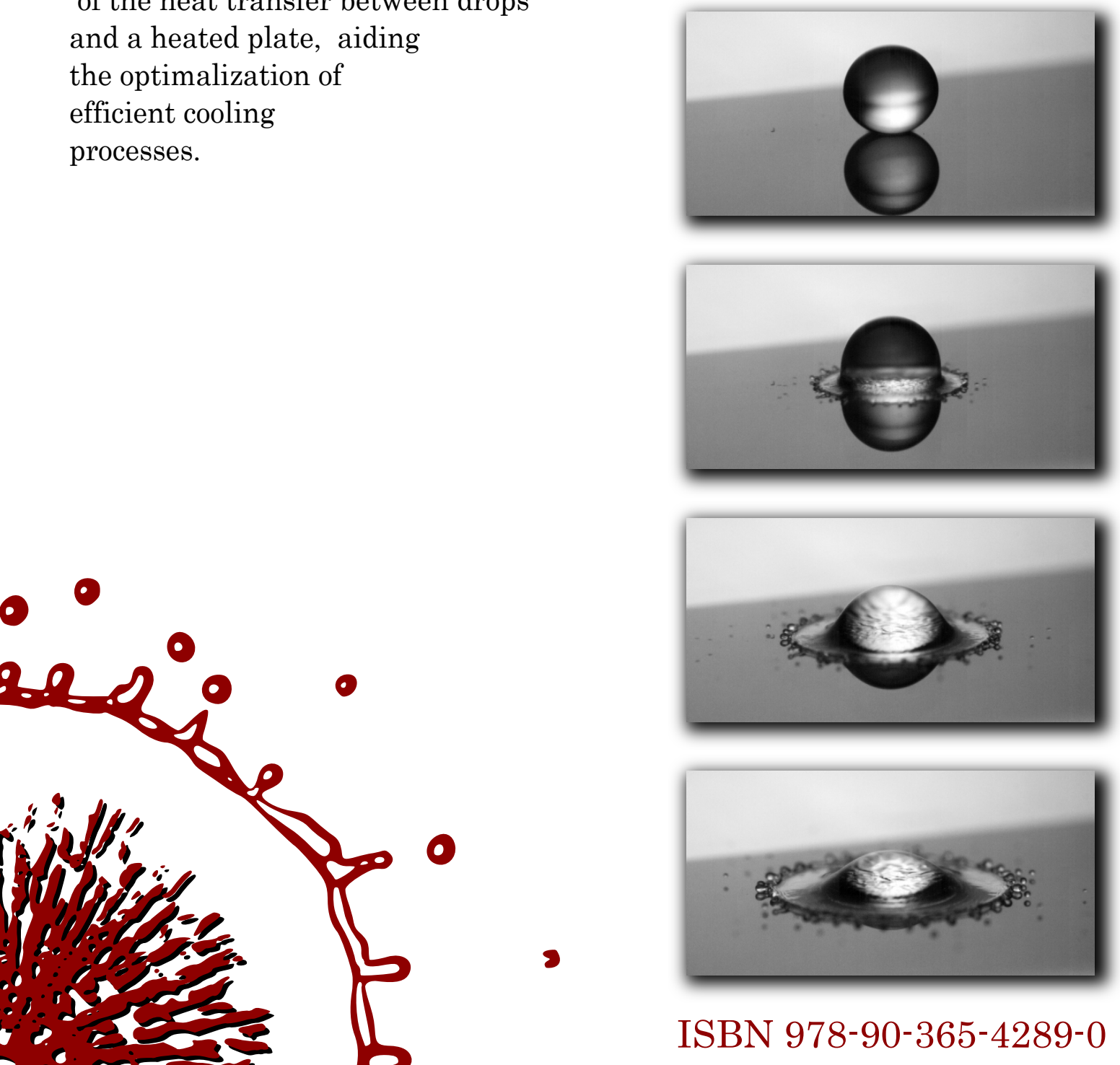